\title{
Isomonodromy Deformations at an Irregular Singularity with Coalescing Eigenvalues
}

\author{
Giordano Cotti, Boris Dubrovin, Davide Guzzetti
}

Giordano Cotti's ORCID ID: 0000-0002-9171-2569

Boris Dubrovin's ORCID ID: 0000-0001-9856-5883

Davide Guzzetti's ORCID ID: 0000-0002-6103-6563

\section{Contents}

1. Introduction 2

2. Deformation of a Differential System with Singularity of the Second Kind 11

3. Fundamental Solutions of (2.13) $\quad 15$

4. A Fundamental Solution of (2.1) at $t=0 \quad 16$

5. Solutions for $t \in \mathcal{U}_{\epsilon_{0}}(0)$ with $A_{0}(t)$ Holomorphically Diagonalisable. $\quad 22$

6. Stokes Phenomenon at $t=0 \quad 27$

7. Stokes Phenomenon at fixed $t_{\Delta} \in \Delta \quad 31$

8. Stokes Phenomenon at $t_{0} \notin \Delta$

9. Stokes Rays rotate as $t$ varies 33

10. Ray Crossing, Wall Crossing and Cell Decomposition 33

11. Sectors $\mathcal{S}_{\nu}(t)$ and $\mathcal{S}_{\nu}(K) \quad 35$

12. Fundamental Solutions $Y_{\nu}(z, t)$ and Stokes Matrices $\mathbb{S}_{\nu}(t)$

13. Analytic Continuation of $Y_{\nu}(z, t)$ on a Cell preserving the Asymptotics 38

14. Fundamental Solutions $Y_{\nu}(z, t)$ and Stokes Matrices $\mathbb{S}_{\nu}(t)$ holomorphic at $\Delta$

15. Meromorphic Continuation 54

16. Structure of Fundamental Solutions in Levelt form at $z=0 \quad 56$

17. Definition of Isomonodromy Deformation of the System (1.9) with Eigenvalues (1.15) 59

18. Isomonodromy Deformation Equations 62

19. Holomorphic Extension of Isomonodromy Deformations to $\mathcal{U}_{\epsilon_{0}}(0)$ and Theorem $1.1 \quad 64$

20. Isomonodromy Deformations with Vanishing Conditions on Stokes Matrices, Proof of $\begin{array}{ll}\text { Theorem } 1.2 & 67\end{array}$

21. Monodromy Data of Semisimple Frobenius Manifolds 75

22. Computation of Monodromy Data of Painlevé Transcendents. Example of the Algebraic Solution associated with $A_{3} \quad 77$

APPENDIX: Examples of Cell Decomposition $\quad 81$

References $\quad 83$

\begin{abstract}
We consider an $n \times n$ linear system of ODEs with an irregular singularity of Poincaré rank 1 at $z=\infty$, holomorphically depending on parameter $t$ within a polydisc in $\mathbb{C}^{n}$ centred at $t=0$. The eigenvalues of the leading matrix at $z=\infty$ coalesce along a locus $\Delta$ contained in the polydisc, passing through $t=0$. Namely, $z=\infty$ is a resonant irregular singularity for $t \in \Delta$. We analyse the case when the leading matrix remains diagonalisable at $\Delta$. We discuss the existence of fundamental matrix solutions, their asymptotics, Stokes phenomenon and monodromy data as $t$ varies in the polydisc, and their limits for $t$ tending to points of $\Delta$. When the deformation is isomonodromic away from $\Delta$, it is well known that a fundamental matrix solution has singularities at $\Delta$. When the system also has a Fuchsian singularity at $z=0$, we show under minimal vanishing conditions on the residue matrix at $z=0$ that isomonodromic deformations can be extended to the whole polydisc, including $\Delta$, in such a way that the fundamental
\end{abstract}


matrix solutions and the constant monodromy data are well defined in the whole polydisc. These data can be computed just by considering the system at fixed $t=0$. Conversely, if the $t$-dependent system is isomonodromic in a small domain contained in the polydisc not intersecting $\Delta$, if the entries of the Stokes matrices with indices corresponding to coalescing eigenvalues vanish, then we show that $\Delta$ is not a branching locus for the fundamental matrix solutions. The importance of these results for the analytic theory of Frobenius Manifolds is explained. An application to Painlevé equations is discussed.

\section{INTRODUCTION}

We study deformations of linear differential systems, playing an important role in applications, with a resonant irregular singularity at $z=\infty$. The $n \times n$ linear (deformed) system depends on parameters $t=\left(t_{1}, \ldots, t_{m}\right) \in \mathbb{C}^{m}$, (here $n, m \in \mathbb{N} \backslash\{0\}$ ) and has the following form:

$$
\frac{d Y}{d z}=A(z, t) Y, \quad A(z, t):=A_{0}(t)+\sum_{k=1}^{\infty} A_{k}(t) z^{-k},
$$

with singularity of Poincaré rank 1 at $z=\infty$. The series $A(z, t)$ is uniformly convergent in a neighborhood of $z=\infty$ for $|z| \geq N_{0}>0$ sufficiently large, and the coefficients $A_{0}(t)$ and $A_{k}(t), k \geq 1$, are holomorphic matrix valued functions on an open connected domain of $\mathbb{C}^{m}$. We take the Poincaré rank equal to 1 in view of the important applications which motivate our work, as it is explained below in this Introduction.

The deformation theory is well understood when $A_{0}(t)$ has distinct eigenvalues $u_{1}(t), u_{2}(t), \ldots, u_{n}(t)$ for $t$ in the domain. On the other hand, there are important cases for applications (see below) when $A_{0}(t)$ is diagonalisable, but two or more eigenvalues may coalesce when $t$ reaches a certain locus $\Delta$ in the $t$-domain, called the coalescence locus. This means that $u_{a}(t)=u_{b}(t)$ for some indices $a \neq b \in\{1, \ldots, n\}$ whenever $t$ belongs to $\Delta$, while $u_{1}(t), u_{2}(t), \ldots, u_{n}(t)$ are pairwise distinct otherwise ${ }^{1}$ Points of $\Delta$ will be called coalescence points. The point $z=\infty$ for $t \in \Delta$ is usually called a resonant irregular singularity, but we will not use this nomenclature throughout the paper. To the best of our knowledge, the analysis of fundamental matrix solutions and their monodromy when $A_{0}(t)$ is diagonalisable with coalescing eigenvalues for $t \in \Delta$, is missing from the existing literature, as we will shortly review later. This is the main problem which we address in the present paper, both in the non-isomonodromic and isomonodromic cases. The main results of the paper are contained in:

- Theorem 14.1, Corollaries 14.1 and 14.2, and in Theorem 15.1, for the non-isomonodromic case;

- Theorem 1.1 (Th. 19.1), Corollary 1.1 (Corol. 19.2) and Theorem 1.2, for the isomonodromic case.

For the sake of the local analysis at coalescence points, we can restrict to the case when the domain is a polydisk

$$
\mathcal{U}_{\epsilon_{0}}(0):=\left\{t \in \mathbb{C}^{m} \quad \text { such that } \quad|t| \leq \epsilon_{0}\right\}, \quad|t|:=\max _{1 \leq i \leq m}\left|t_{i}\right|
$$

for suitable $\epsilon_{0}>0$, being $t=0$ a point of the coalescence locus. We will again denote by $\Delta$ the coalescence locus in $\mathcal{U}_{\epsilon_{0}}(0)$. It is well known that the eigenvalues $u_{1}(t), \ldots, u_{n}(t)$ are branches of one or more functions of $t$, with algebraic branching at $\Delta$ (see [45]). A matrix $G_{0}(t)$ which diagonalises $A_{0}(t)$ for $t \notin \Delta$, namely such that $G_{0}^{-1}(t) A_{0}(t) G_{0}(t)=\Lambda(t)$, where

$$
\Lambda(t):=\operatorname{diag}\left(u_{1}(t), \ldots, u_{n}(t)\right),
$$

is generally singular when $t$ approaches $\Delta$. Example will be given in Section 2.1. Consider a fundamental solution $^{2}$ that for $t$ belonging to a sufficiently small domain $\mathcal{V} \subset \mathcal{U}_{\epsilon_{0}}(0)$, with $\mathcal{V} \cap \Delta=\emptyset$, has a canonical asymptotic representation (see [37])

$$
Y(z, t) \sim G_{0}(t)\left(I+\sum_{k=1}^{\infty} F_{k}(t) z^{-k}\right) z^{B_{1}} e^{\Lambda(t) z}, \quad z \rightarrow \infty,
$$

\footnotetext{
${ }^{1} \Delta$ is a discrete set for $m=1$, otherwise it is a continuous locus for $m \geq 2$. For example, for the matrix $\operatorname{diag}\left(t_{1}, t_{2}, \ldots, t_{n}\right)$, the coalescence locus is the union of the diagonals $t_{i}=t_{j}, i \neq j \in\{1,2, \ldots, n\}$.

${ }^{2} \mathrm{~A}$ fundamental matrix solution will be simply called a fundamental solution.
} 
in a suitable sector $\mathcal{S}(\overline{\mathcal{V}})$ depending on $\mathcal{V}$, explained after formula (1.6) below. Here $I$ stands for the identity matrix and $B_{1}$ is a diagonal matrix, given in formula (1.6). Then, the $t$-analytic continuation of $Y(z, t)$ inherits the singularities of $G_{0}(t)$ as $t$ tends to $\Delta$. Thus, in order to extend the deformation theory when $t$ approaches $\Delta$, we need the following:

Assumption 1: The holomorphic matrix $A_{0}(t)$ is holomorphically similar in $\mathcal{U}_{\epsilon_{0}}(0)$ to a diagonal matrix $\Lambda(t)$ as in (1.2), namely there exists an invertible matrix $G_{0}(t)$ holomorphic on $\mathcal{U}_{\epsilon_{0}}(0)$ such that

$$
G_{0}^{-1}(t) A_{0}(t) G_{0}(t)=\Lambda(t) .
$$

Assumption 1, which is basically the assumption of the paper, holds for example for Frobenius manifolds remaining semisimple at the locus of coalescent canonical coordinates, and in applications to the sixth Painlevé transcendents holomorphic at a fixed singularity of the Painlevé equation (see Sections 21 and 22 below).

Given Assumption 1, the transformation $Y \mapsto G_{0}(t) Y$ changes $A(z, t)$ to a matrix valued function

$$
\widehat{A}(z, t):=G_{0}(t)^{-1} A(z, t) G_{0}(t),
$$

holomorphic on $\left\{|z| \geq N_{0}\right\} \times \mathcal{U}_{\epsilon_{0}}(0)$ for sufficiently large $N_{0}>0$, so that system (1.1) becomes

$$
\frac{d Y}{d z}=\widehat{A}(z, t) Y, \quad \widehat{A}(z, t)=\Lambda(t)+\sum_{k=1}^{\infty} \widehat{A}_{k}(t) z^{-k}
$$

where $\widehat{A}_{k}(t), k \geq 1$, and $\Lambda(t)$ are holomorphic on $\mathcal{U}_{\epsilon_{0}}(0)$.

When $\Delta$ is not empty, the dependence on $t$ of fundamental solutions of (1.5) near $z=\infty$ is quite delicate. If $t \notin \Delta$, then the system (1.5) has a unique formal solution (see [37]),

$$
Y_{F}(z, t):=\left(I+\sum_{k=1}^{\infty} F_{k}(t) z^{-k}\right) z^{B_{1}(t)} e^{\Lambda(t) z}, \quad B_{1}(t):=\operatorname{diag}\left(\widehat{A}_{1}(t)\right),
$$

where the matrices $F_{k}(t)$ are uniquely determined by the equation and are holomorphic on $\mathcal{U}_{\epsilon_{0}}(0) \backslash \Delta$.

In order to find actual solutions, and their domain of definition in the space of parameters $t$, one can refer to the local existence results of Sibuya [66] [37] (see Theorems 2.1 and 5.1 below), which guarantees that, given $t_{0} \in \mathcal{U}_{\epsilon_{0}}(0) \backslash \Delta$, there exists a sector and a fundamental solution $Y(z, t)$ holomorphic for $|z|$ large and $\left|t-t_{0}\right|<\rho$, where $\rho$ is sufficiently small, such that $Y(z, t) \sim Y_{F}(z, t)$ for $z \rightarrow \infty$ in the sector. The condition $\left|t-t_{0}\right|$ is restrictive, since $\rho$ is expected to be very small. In the present paper, we prove this result for $t$ in a wider domain $\mathcal{V} \subset \mathcal{U}_{\epsilon_{0}}(0)$, extending $\left|t-t_{0}\right|<\rho$. $\mathcal{V}$ is constructed as follows. Let $t=0$ and consider the Stokes rays associated with the matrix $\Lambda(0)$, namely rays in the universal covering $\mathcal{R}$ of the $z$-punctured plane $\mathbb{C} \backslash\{0\}$, defined by the condition that $\Re e\left[\left(u_{a}(0)-u_{b}(0)\right) z\right]=0$, with $u_{a}(0) \neq u_{b}(0)(1 \leq a \neq b \leq n)$. Then, consider an admissible ray, namely a ray in $\mathcal{R}$, with a certain direction $\widetilde{\tau}$, that does not contain any of the Stokes rays above, namely $\Re e\left[\left(u_{a}(0)-u_{b}(0)\right) z\right] \neq 0$ for any $u_{a}(0) \neq u_{b}(0)$ and $\arg z=\widetilde{\tau}$. Define the locus $X(\widetilde{\tau})$ to be the set of points $t \in \mathcal{U}_{\epsilon_{0}}(0)$ such that some Stokes rays $\left\{z \in \mathcal{R} \mid \Re e\left[\left(u_{a}(t)-u_{b}(t)\right) z\right]=0\right\}$ associated with $\Lambda(t), t \notin \Delta$, coincide with the admissible ray $\arg z=\tilde{\tau}$. Finally, define a $\widetilde{\tau}$-cell to be any connected component of $\mathcal{U}_{\epsilon_{0}}(0) \backslash(\Delta \cup X(\widetilde{\tau}))$ (see Section 10 for a thorough study of the cells). Then, we take an open connected open domain $\mathcal{V}$ such that its closure $\overline{\mathcal{V}}$ is contained in a $\widetilde{\tau}$-cell.

Definition 1.1. The deformation of the linear system (1.9), such that $t$ varies in an open connected domain $\mathcal{V} \subset \mathcal{U}_{\epsilon_{0}}(0)$, such $\overline{\mathcal{V}}$ is contained in a $\widetilde{\tau}$-cell, is called an admissible deformation ${ }^{3}$. For simplicity, we will just say that $t$ is an admissible deformation.

By definition, an admissible deformation means that as long as $t$ varies within $\overline{\mathcal{V}}$, then no Stokes rays of $\Lambda(t)$ cross the admissible ray of direction $\widetilde{\tau}$.

If $t$ belongs to a domain $\mathcal{V}$ as above, then we prove in Section 13 that there is a family of actual fundamental solutions $Y_{r}(z, t)$, labelled by $r \in \mathbb{Z}$, uniquely determined by the canonical asymptotic

\footnotetext{
${ }^{3}$ The definition of admissible deformation of a linear system is in accordance with the definition given in [28].
} 

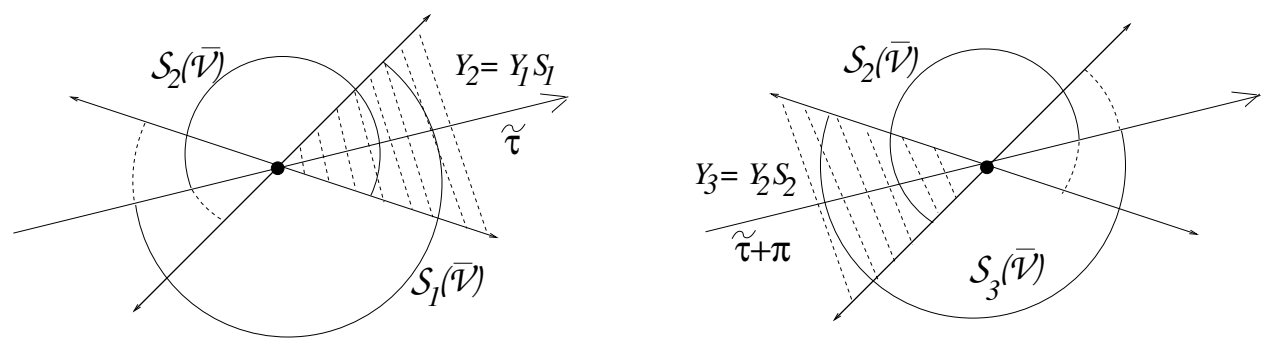

FiguRE 1. Stokes phenomenon of formula (1.7). In the left figure is represented the sheet of the universal covering $\widetilde{\tau}-\pi<\arg z<\widetilde{\tau}+\pi$ containing $\mathcal{S}_{1}(\overline{\mathcal{V}}) \cap \mathcal{S}_{2}(\overline{\mathcal{V}})$, and in the right figure the sheet $\widetilde{\tau}<\arg z<\widetilde{\tau}+2 \pi$ containing $\mathcal{S}_{2}(\overline{\mathcal{V}}) \cap \mathcal{S}_{3}(\overline{\mathcal{V}})$. The rays $\arg z=\widetilde{\tau}$ and $\widetilde{\tau}+\pi$ (and then $\widetilde{\tau}+k \pi$ for any $k \in \mathbb{Z}$ ) are admissible rays, such that $\Re e\left[\left(u_{a}(0)-u_{b}(0)\right) z\right] \neq 0$ along these rays, for any $u_{a}(0) \neq u_{b}(0)$. Moreover, $\Re e\left[\left(u_{a}(t)-\right.\right.$ $\left.\left.u_{b}(t)\right) z\right] \neq 0$ for any $t \in \mathcal{V}$ and any $1 \leq a \neq b \leq n$.

representation

$$
Y_{r}(z, t) \sim Y_{F}(z, t)
$$

for $z \rightarrow \infty$ in suitable sectors $\mathcal{S}_{r}(\overline{\mathcal{V}})$ of the universal covering $\mathcal{R}$ of $\mathbb{C} \backslash\{0\}$. Each $Y_{r}(z, t)$ is holomorphic in $\{z \in \mathcal{R}|| z \mid \geq N\} \times \mathcal{V}$, for a suitably large $N$. The asymptotic series $I+\sum_{k=1}^{\infty} F_{k}(t) z^{-k}$ is uniform in $\overline{\mathcal{V}}$.

The sectors $\mathcal{S}_{r}(\overline{\mathcal{V}})$ are constructed as follows: take for example the " half plane" $\Pi_{1}:=\{z \in \mathcal{R} \mid \widetilde{\tau}-\pi<$ $\arg z<\widetilde{\tau}$. The open sector containing $\Pi_{1}$ and extending up to the closest Stokes rays of $\Lambda(t)$ outside $\Pi_{1}$ will be called $\mathcal{S}_{1}(t)$. Then, we define $\mathcal{S}_{1}(\overline{\mathcal{V}}):=\bigcap_{t \in \overline{\mathcal{V}}} \mathcal{S}_{1}(t)$. Analogously, we consider the "half-planes" $\Pi_{r}:=\{z \in \mathcal{R} \mid \widetilde{\tau}+(r-3) \pi<\arg z<\widetilde{\tau}+(r-1) \pi\}$ and repeat the same construction for $\mathcal{S}_{r}(\overline{\mathcal{V}})$. The sectors $\mathcal{S}_{r}(\overline{\mathcal{V}})$ have central opening angle greater than $\pi$ and their successive intersections do not contain Stokes rays $\Re e\left[\left(u_{a}(t)-u_{b}(t)\right) z\right]=0$ associated with the eigenvalues of $\Lambda(t), t \in \overline{\mathcal{V}}$. The sectors $\mathcal{S}_{r}(\overline{\mathcal{V}})$ for $r=1,2,3$ are represented in Figure 1. An admissible ray $\arg z=\widetilde{\tau}$ in $\mathcal{S}_{1}(\overline{\mathcal{V}}) \cap \mathcal{S}_{2}(\overline{\mathcal{V}})$ is also represented.

If the $t$-analytic continuation of $Y_{r}(z, t)$ exists outside $\mathcal{V}$, then the delicate points emerge, as follows.

- The expression $\Re e\left[\left(u_{a}(t)-u_{b}(t)\right) z\right], 1 \leq a \neq b \leq n$, has constant sign in the $\widetilde{\tau}$-cell containing $\mathcal{V}$, but it vanishes when a Stokes ray $\Re e\left[\left(u_{a}(t)-u_{b}(t)\right) z\right]=0$ crosses the admissible direction $\widetilde{\tau}$. This corresponds to the fact that $t$ crosses the boundary of the cell. Then, it changes sign for $t$ outside of the cell. Hence, the asymptotic representation $Y_{r}(z, t) \sim Y_{F}(z, t)$ for $z \rightarrow \infty$ in $\mathcal{S}_{r}(\overline{\mathcal{V}})$ does no longer hold for $t$ outside the $\widetilde{\tau}$-cell containing $\mathcal{V}$.

- The coefficients $F_{k}(t)$ are in general divergent at $\Delta$.

- The locus $\Delta$ is expected to be a locus of singularities for the $Y_{r}(z, t)$ 's (see Example 5.1 below).

- The Stokes matrices $\mathbb{S}_{r}(t)$, defined for $t \in \mathcal{V}$ by the relations (see Figure 1)

$$
Y_{r+1}(z, t)=Y_{r}(z, t) \mathbb{S}_{r}(t),
$$

are expected to be singular as $t$ approaches $\Delta$.

Remark 1.1. It is well known that, in order to completely describe the Stokes phenomenon, it suffices to consider only three fundamental solutions, for example $Y_{r}(z, t)$ for $r=1,2,3$, and $\mathbb{S}_{1}(t), \mathbb{S}_{2}(t)$.

The matrix $A(z, t)$ may have other singularities at finite values of $z$. In the isomonodromic case, we will consider $A(z, t)$ with a simple pole at $z=0$, namely

$$
A(z, t)=A_{0}(t)+\frac{A_{1}(t)}{z} .
$$

An isomonodromic system of type (1.8), with antisymmetric $A_{1}$, is at the core of the analytic approach to semisimple Frobenius manifolds [17] [18] [19] (see also [62] [63] [64] [53] [61]). Its monodromy data play the role of local moduli. Coalescence of eigenvalues of $A_{0}(t)$ occurs in important cases, such as quantum cohomology (see [14] [15] and Section 21 below). For $n=3$, a special case of system (1.8) 
gives an isomonodromic description of the general sixth Painlevé equation, according to [54] (see also [35]). This description was given also in [17] [19] for a sixth Painlevé equation associated with Frobenius manifolds. Coalescence occurs at the critical points of the Painlevé equation (see Section 22).

The transformation $Y \mapsto G_{0}(t) Y$ changes (1.8) into

$$
\frac{d Y}{d z}=\widehat{A}(z, t) Y, \quad \widehat{A}(z, t)=\Lambda(t)+\frac{\widehat{A}_{1}(t)}{z} .
$$

For given $t$, a matrix $G^{(0)}(t)$ (not to be confused with $G_{0}(t)$ in $(1.3)$ ), puts $\widehat{A}_{1}(t)$ in Jordan form

$$
J^{(0)}(t):=\left(G^{(0)}(t)\right)^{-1} \widehat{A}_{1}(t) G^{(0)}(t) .
$$

Close to the Fuchsian singularity $z=0$, and for a given $t$, the system (1.9) has a fundamental solution

$$
Y^{(0)}(z, t)=G^{(0)}(t)\left(I+\sum_{l=1}^{\infty} \Psi_{l}(t) z^{l}\right) z^{D^{(0)}(t)} z^{S^{(0)}(t)+R^{(0)}(t)},
$$

in standard Birkhoff-Levelt normal form, whose behaviour in $z$ and $t$ is not affected by the coalescence phenomenon. The matrix coefficients $\Psi_{l}(t)$ of the convergent expansion are constructed by a recursive procedure. $D^{(0)}(t)=\operatorname{diag}\left(d_{1}(t), \ldots, d_{n}(t)\right)$ is a diagonal matrix of integers, piecewise constant in $t, S^{(0)}(t)$ is a Jordan matrix whose eigenvalues $\rho_{1}(t), \ldots, \rho_{n}(t)$ have real part in $[0,1[$, and the nilpotent matrix $R^{(0)}(t)$ has non-vanishing entries only if some eigenvalues of $\widehat{A}_{1}(t)$ differ by non-zero integers. If some eigenvalues differ by non-zero integers, we say that $\widehat{A}_{1}(t)$ is resonant. The sum

$$
J^{(0)}(t)=D^{(0)}(t)+S^{(0)}(t)
$$

is the Jordan form of $\widehat{A}_{1}(t)$ above. Under the assumptions of our Theorem 1.1 below, the solution (1.10) turns out to be holomorphic in $t \in \mathcal{U}_{\epsilon_{0}}(0)$. ${ }^{4}$

In order to completely describe the monodromy of the system (1.9), we need its essential monodromy data (the adjective "essential" is inspired by a similar definition in [44]). We recall that it suffices to consider three fundamental solutions, for example $Y_{r}(z, t)$ for $r=1,2,3$, and consequently the Stokes matrices $\mathbb{S}_{1}(t)$ and $\mathbb{S}_{2}(t)$. Moreover, chosen a solution $Y^{(0)}(z, t)$ with normal form (1.10), a central connection matrix $C^{(0)}$ is defined by the relation

$$
Y_{1}(z, t)=Y^{(0)}(z, t) C^{(0)}(t), \quad z \in \mathcal{S}_{1}(\overline{\mathcal{V}}) .
$$

Then, the essential monodromy data of the system (1.9) are defined to be

$$
\mathbb{S}_{1}(t), \quad \mathbb{S}_{2}(t), \quad B_{1}(t)=\operatorname{diag}\left(\widehat{A}_{1}(t)\right), \quad C^{(0)}(t), \quad J^{(0)}(t), \quad R^{(0)}(t) .
$$

Now, when $t$ tends to a point $t_{\Delta} \in \Delta$, the limits of the above data may not exist. If the limits exist, they do not in general give the monodromy data of the system $\widehat{A}\left(z, t_{\Delta}\right)$. The latter have in general different nature, as it is clear from the results of [3], and from Section 3 below. ${ }^{5}$

Definition 1.2. If the deformation is admissible in a domain $\mathcal{V}$, as in Definition 1.1, we say that it is isomonodromic in $\mathcal{V}$ if the essential monodromy data (1.12) do not depend on $t \in \mathcal{V}$.

When this definition holds, the classical theory of Jimbo-Miwa-Ueno [44] applies. ${ }^{6}$ We are interested in extending the deformation theory to the whole $\mathcal{U}_{\epsilon_{0}}(0)$, including the coalescence locus $\Delta$.

\footnotetext{
${ }^{4}$ If $Y^{(0)}(z, t)$ is chosen, with given $G^{(0)}(t), \Psi_{l}(t)$ 's and $R^{(0)}(t)$, then there is a class of suitable matrices $\mathfrak{D}(t)$ such that $Y^{(0)}(z, t) \mathfrak{D}(t)$ also has the standard form (1.10) with new $G^{(0)}(t), \Psi_{l}(t)$ 's and $R^{(0)}(t)$. More details are in Section 16 .

${ }^{5}$ See for example the solution (4.13), where it is evident that the monodromy datum $L$, defined at $t=0$, is not the limit for $t \rightarrow 0$ of $B_{1}(t)$ as in (1.6).

${ }^{6}$ Notice that in [44] it is also assumed that $A_{1}(t)$ is diagonalisable with eigenvalues not differing by integers. We do not make this assumption here.
} 
1.1. Main Results. a] The case of systems (1.1) and (1.5). Up to Section 15, we study system (1.1) without requiring that the deformation is isomonodromic. First, we give the general formal and actual solutions for $z \rightarrow \infty$ of system (1.1) when $t=0$ (or $t_{\Delta} \in \Delta$ ), without Assumption 1. ${ }^{7}$ Then, under Assumption 1, in Proposition 5.1 we give necessary and sufficient conditions such that the coefficients $F_{k}(t)$ of a formal solution of (1.1)

$$
Y_{F}(z, t)=G_{0}(t)\left(I+\sum_{k=1}^{\infty} F_{k}(t) z^{-k}\right) z^{B_{1}(t)} e^{\Lambda(t) z}, \quad t \notin \Delta,
$$

are actually holomorphic also at $t \in \Delta$. Notice that our result cannot be derived from [1] and [65], where holomorphic confluence for $t \rightarrow 0$ of formal solutions is studied, since $\Lambda(t) z$ is in general not "wellbehaved" (condition (4.2) of [65] is violated). In Section 13, we prove that fundamental solutions $Y_{r}(z, t)$, $r \in \mathbb{Z}$, of (1.1) can be $t$-analytically continued to a whole $\widetilde{\tau}$-cell containing the domain $\mathcal{V}$ of Definition 1.1, preserving the asymptotic representation (1.13). In Theorem 14.1 we give sufficient conditions such that fundamental solutions $Y_{r}(z, t)$, together with their Stokes matrices $\mathbb{S}_{r}(t)$, are actually holomorphic also at $\Delta$ and in the whole $\mathcal{U}_{\epsilon_{0}}(0)$, in such a way that the asymptotic representation $Y_{r}(z, t) \sim Y_{F}(z, t)$ continues to hold, for $z \rightarrow \infty$ in wider sectors $\widehat{\mathcal{S}}_{r}$ containing $\mathcal{S}_{r}(\overline{\mathcal{V}})$, to be introduced below (see (1.19)). We show in this case that the limits

$$
\lim _{t \rightarrow t_{\Delta}} \mathbb{S}_{r}(t), \quad t_{\Delta} \in \Delta
$$

exist and are finite. They give the Stokes matrices for the system (1.1) with matrix coefficient $A\left(z, t_{\Delta}\right)$ (see Corollary 14.1 and 14.2). In the analysis of the above issues, wall crossing phenomena and cell decompositions of $\mathcal{U}_{\epsilon_{0}}(0)$ will be studied. Another result on the analytic ocntinuation of fundamental solutions, with vanishing conditions on the Stokes matrices, is given in Theorem 15.1.

We compare our results with the existing literature, where sometimes the irregular singular point is taken at $z=0$ (equivalent to $z=\infty$ by a change $z \mapsto 1 / z$ ). One considers a "folded" system $A(z, 0)=z^{-k-1} \sum_{j=0}^{\infty} A_{j}(0) z^{j}$, with an irregular singularity of Poincaré rank $k$ at $z=0$ and studies its holomorphic unfolding $A(z, t)=p(z, t)^{-1} \sum_{j=0}^{\infty} A_{j}(t) z^{j}$, where $p(z, t)=\left(z-a_{1}(t)\right) \cdots\left(z-a_{k+1}(t)\right)$ is a polynomial. Early studies on the relation between monodromy data of the "folded" and the "unfolded" systems were started by Garnier [29], and the problem was again raised by V.I. Arnold in 1984 and studied by many authors in the '80's and '90's of the XX century, for example see [59], [26], [9]. Under suitable conditions, some results have been recently established regarding the convergence for $t \rightarrow 0$ ( $t$ in sectors or suitable ramified domains) of fundamental solutions and monodromy data (transition or connection matrices) of the "unfolded" system to the Stokes matrices of the "folded" one [59], [26], [9], [1], [65], [30], [31], [38], [40], [46]. Nevertheless, to our knowledge, the case when $A_{0}(0)$ is diagonalisable with coalescing eigenvalues has not yet been studied. For example, in [30] (see also references therein) and [38] [40], it is assumed that the leading matrix $A_{0}(0)$ has distinct eigenvalues. In [31], $A_{0}(0)$ is a single $n \times n$ Jordan block (only one eigenvalue), with a generic condition on $A(z, t)$. Moreover, the irregular singular point is required to split into non-resonant Fuchsian singularities $a_{1}(t), \ldots, a_{k+1}(t)$. The case when $A_{0}(0)$ is a $2 \times 2$ Jordan block and $k=1$ is thoroughly described in [46], again under a generic condition on $A(z, t)$, with no conditions on the polynomial $p(z, t)$. Explicit normal forms for the unfolded systems are given (including an explanation of the change of order of Borel summability when $z=0$ becomes a resonant irregular singularity as $t \rightarrow 0$ ). Nevertheless, both in [31] and [46] the system at $t=0$ is ramified and the fundamental matrices $Y_{r}(z, t)$ diverge when $t \rightarrow 0$, together with the corresponding Stokes matrices. Therefore, our results on the extension of the asymptotic representation at $\Delta$ and the existence of the limit (1.14), for a system with diagonalisable $A_{0}\left(t_{\Delta}\right)$, seem to be missing in the literature.

b] Isomonodromic case of system (1.9). Let the deformation be isomonodromic in $\mathcal{V}$, as in Definition 1.2, so that the classical theory of Jimbo-Miwa-Ueno applies. As a result of [44], the

\footnotetext{
${ }^{7}$ We give an explicit construction of the formal solutions; their structure can also be derived from [3].
} 
eigenvalues can be chosen as the independent deformation parameters. This means that we can assume ${ }^{8}$ linearity in $t \in \mathcal{U}_{\epsilon_{0}}(0)$, as follows:

$$
u_{a}(t)=u_{a}(0)+t_{a}, \quad 1 \leq a \leq n \quad \Longrightarrow m=n .
$$

Therefore,

$$
\Lambda(t)=\Lambda(0)+\operatorname{diag}\left(t_{1}, \ldots, t_{n}\right)
$$

with

$$
\Lambda(0)=\Lambda_{1} \oplus \cdots \oplus \Lambda_{s}, \quad s<n, \quad \Lambda_{i}=\lambda_{i} I_{p_{i}},
$$

where $\lambda_{1}, \ldots, \lambda_{s}$ are the $s<n$ distinct eigenvalues of $\Lambda(0)$, of respectively multiplicities $p_{1}, \ldots, p_{s}\left(p_{1}+\right.$ $\left.\cdots+p_{s}=n\right)$. Here, $I_{p_{i}}$ is the $p_{i} \times p_{i}$ identity matrix. Now, the size $\epsilon_{0}$ of $\mathcal{U}_{\epsilon_{0}}(0)$ is taken sufficiently small so that we can write

$$
\Lambda(t)=\Lambda_{1}(t) \oplus \cdots \oplus \Lambda_{s}(t),
$$

with the properties that $\lim _{t \rightarrow 0} \Lambda_{j}(t)=\lambda_{j} I_{p_{j}}$, and that $\Lambda_{i}(t)$ and $\Lambda_{j}(t)$ have no common eigenvalues for $i \neq j$. Thus, $\Delta$ is represented as

$$
\Delta=\bigcup_{i=1}^{s} \Delta_{i}
$$

where

$$
\Delta_{i}:=\left\{t \in \mathcal{U}_{\epsilon_{0}}(0) \mid t_{a}=t_{b} \text { with } u_{a}(0)=u_{b}(0)=\lambda_{i}\right\} .
$$

Our problem is to extend the isomonodromy deformation theory from $\mathcal{V}$ to the whole $\mathcal{U}_{\epsilon_{0}}(0)$ in this case.

As it will be reviewed below after Theorem 1.2, the existing literature on isomonodromy deformations does not seem to solve our problem. We give a solution in the following Theorem 1.1 and Corollary 1.1 (equivalently, see Theorem 19.1 and Corollary 19.2 in the main body of the paper).

In order state Theorem 1.1 in a precise way, we need a last technical remark on the radius $\epsilon_{0}$ of the polydisc. As explained above, $\epsilon_{0}$ is sufficiently small to ensure that $\Lambda_{i}(t)$ has no eigenvalues in common with $\Lambda_{j}(t)$, for $i \neq j$ (see (1.17)). Moreover, we require that it satisfies the following constraint

$$
\epsilon_{0}<\min _{1 \leq j \neq k \leq s} \delta_{j k}
$$

where

$$
\delta_{j k}:=\frac{1}{2} \min _{\rho \in \mathbb{R}}\left\{\left|\lambda_{k}-\lambda_{j}+i \rho \exp \{-i \tilde{\tau}\}\right|\right\}
$$

(here $i$ is the imaginary unit). This condition has a geometrical reason. If we represent $\lambda_{1}, \ldots, \lambda_{s}$ in the same $\lambda$-plane, we can easily verify that the distance between the two parallel lines through $\lambda_{j}$ and $\lambda_{k}$ of angular direction $3 \pi / 2-\widetilde{\tau}$ is exactly $2 \delta_{j k}$. Let us consider Stokes rays $\left\{z \in \mathcal{R} \mid \Re\left(z\left(u_{a}(t)-u_{b}(t)\right)=0\right\}\right.$ associated with couples $u_{a}(t), u_{b}(t), a, b \in\{1,2, \ldots, n\}$, such that $u_{a}(0)=\lambda_{j}$ and $u_{b}(0)=\lambda_{k}$, with $1 \leq j \neq k \leq s$. None of these rays crosses the admissible directions $\widetilde{\tau}+k \pi, k \in \mathbb{Z}$, when $t$ varies in $\mathcal{U}_{\epsilon_{0}}(0)$ with $\epsilon_{0}$ as in (1.18). For a given $t$, let $\mathfrak{R}(t)$ be the set of all the above rays for all $j \neq k$. We construct a sector $\widehat{\mathcal{S}}_{r}(t)$ containing the "half-plane" $\Pi_{r}$ (defined above), and extending up to the closest Stokes rays of $\mathfrak{R}(t)$ lying outside $\Pi_{r}$. Clearly, $\widehat{\mathcal{S}}_{r}(t) \supset \mathcal{S}_{r}(t)$. Then, define

$$
\widehat{\mathcal{S}}_{r}:=\bigcap_{t \in \mathcal{U}_{\epsilon_{0}}(0)} \widehat{\mathcal{S}}_{r}(t)
$$

By construction, if $\epsilon_{0}$ is as in (1.18), then this sector has central opening angle greater than $\pi$. Note that $\mathcal{S}_{r}(\overline{\mathcal{V}}) \subseteq \widehat{\mathcal{S}}_{r}$

Theorem 1.1. Consider the system (1.9), with eigenvalues of $\Lambda(t)$ linear in $t$ as in (1.15), and with $A_{1}(t)$ holomorphic on a closed polydisc $\mathcal{U}_{\epsilon_{0}}(0)$ centred at $t=0$, with sufficiently small radius $\epsilon_{0}$ as in (1.18). Let $\Delta$ be the coalescence locus in $\mathcal{U}_{\epsilon_{0}}(0)$, passing through $t=0$. Let the dependence on $t$ be isomonodromic in a domain $\mathcal{V}$ as in Definition 1.2.

\footnotetext{
8 This assumption will be used in the paper starting from Section 14.2.
} 
If the matrix entries of $\widehat{A}_{1}(t)$ satisfy in $\mathcal{U}_{\epsilon_{0}}(0)$ the vanishing conditions

$$
\left(\widehat{A}_{1}(t)\right)_{a b}=\mathcal{O}\left(u_{a}(t)-u_{b}(t)\right), 1 \leq a \neq b \leq n,
$$

whenever $u_{a}(t)$ and $u_{b}(t)$ coalesce as $t$ tends to a point of $\Delta$, then the following results hold:

- The formal solution $Y_{F}(z, t)$ of (1.9) as given in (1.6) is holomorphic on the whole $\mathcal{U}_{\epsilon_{0}}(0)$.

- The three fundamental matrix solutions $Y_{r}(z, t), r=1,2,3$, of the system of (1.9), which are defined on $\mathcal{V}$, with asymptotic representation $Y_{F}(z, t)$ for $z \rightarrow \infty$ in sectors $\mathcal{S}_{r}(\overline{\mathcal{V}})$ introduced above, can be t-analytically continued as single-valued holomorphic functions on $\mathcal{U}_{\epsilon_{0}}(0)$, with asymptotic representation

$$
Y_{r}(z, t) \sim Y_{F}(z, t), \quad z \rightarrow \infty \text { in } \widehat{\mathcal{S}}_{r},
$$

for any $t \in \mathcal{U}_{\epsilon_{1}}(0)$, and any $0<\epsilon_{1}<\epsilon_{0}$. In particular, they are defined at any $t_{\Delta} \in \Delta$ with asymptotic representation $Y_{F}\left(z, t_{\Delta}\right)$. The fundamental matrix solution $Y^{(0)}(z, t)$ is also $t$-analytically continued as a single-valued holomorphic function on $\mathcal{U}_{\epsilon_{0}}(0)$

- The constant Stokes matrices $\mathbb{S}_{1}, \mathbb{S}_{2}$, and a central connection matrix $C^{(0)}$, initially defined for $t \in \mathcal{V}$, are actually globally defined on $\mathcal{U}_{\epsilon_{0}}(0)$. They coincide with the Stokes and connection matrices of the fundamental solutions $Y_{r}(z, 0)$ and $Y^{(0)}(z, 0)$ of the system

$$
\frac{d Y}{d z}=\widehat{A}(z, 0) Y, \quad \widehat{A}(z, 0)=\Lambda(0)+\frac{\widehat{A}_{1}(0)}{z} .
$$

Also the remaining $t$-independent monodromy data in (1.12) coincide with those of (1.21).

- The entries $(a, b)$ of the Stokes matrices are characterised by the following vanishing property:

$$
\left(\mathbb{S}_{1}\right)_{a b}=\left(\mathbb{S}_{1}\right)_{b a}=\left(\mathbb{S}_{2}\right)_{a b}=\left(\mathbb{S}_{2}\right)_{b a}=0 \quad \text { whenever } u_{a}(0)=u_{b}(0), \quad 1 \leq a \neq b \leq n \text {. }
$$

Theorem 1.1 allows to holomorphically define the fundamental solutions and the monodromy data on the whole $\mathcal{U}_{\epsilon_{0}}(0)$, under the only condition (1.20). This fact is remarkable. Indeed, according to [55], in general the solutions $Y^{(0)}(z, t), Y_{r}(z, t)$ and $\widehat{A}(z, t), t \in \mathcal{V}$, of monodromy preserving deformation equations can be analytically continued as meromorphic matrix valued functions on the universal covering of $\mathbb{C}^{n} \backslash \Delta_{\mathbb{C}^{n}}$, where $\Delta_{\mathbb{C}^{n}}=\bigcup_{a \neq b}^{n}\left\{u_{a}(t)=u_{b}(t)\right\}$ is the coalescence locus in $\mathbb{C}^{n}$. They have fixed singularities at the branching locus $\Delta_{\mathbb{C}^{n}}$, and so at $\Delta \subset \Delta_{\mathbb{C}^{n}}$. Moreover, the $t$-analytic continuation on $\mathcal{U}_{\epsilon_{0}}(0)$ of a the solutions $Y_{r}(z, t)$ are expected to lose their asymptotic representation $Y_{r}(z, t) \sim Y_{F}(z, t)$ in $\mathcal{S}_{r}(\overline{\mathcal{V}})$, when $t$ moves sufficiently far away from $\mathcal{V}$, namely when Stokes rays cross and admissible ray of direction $\widetilde{\tau}$. Under the assumptions of Theorem 1.1 these singular behaviours do not occur.

Let the assumptions of Theorem 1.1 hold. Then, the system (1.21) has a formal solutions (here we denote objects $Y, \mathbb{S}$ and $C$ referring to the system (1.21) with the symbols ${ }^{Y}$, $\stackrel{\circ}{\mathbb{S}}$ and $\stackrel{\circ}{C}$ ) with behaviour ${ }^{9}$

$$
\stackrel{\circ}{Y}_{F}(z)=\left(I+\sum_{k=1}^{\infty} \stackrel{\circ}{F}_{k} z^{-k}\right) z^{B_{1}(0)} e^{\Lambda(0) z}, \quad B_{1}(0)=\operatorname{diag}\left(\widehat{A}_{1}(0)\right) .
$$

The matrix-coefficients $\stackrel{\circ}{F}_{k}$ are recursively constructed from the equation (1.21), but not uniquely determined. Actually, there is a family of formal solutions as above, depending on a finite number of complex parameters. To each element of the family, there correspond unique actual solutions $Y_{1}(z), \stackrel{\circ}{2}_{2}(z), \dot{\Upsilon}_{3}(z)$ such that $\stackrel{\circ}{Y}_{r}(z) \sim \stackrel{\circ}{Y}_{F}(z)$ for $z \rightarrow \infty$ in a sector $\mathcal{S}_{r} \supset \mathcal{S}_{r}(\overline{\mathcal{V}}), r=1,2,3$, with Stokes matrices defined by

$$
\stackrel{\circ}{Y+1}_{r+1}(z)=\stackrel{\circ}{Y}(z) \stackrel{\circ}{\mathbb{S}}_{r}, \quad r=1,2 .
$$

Only one element of the family of formal solutions (1.23) satisfies the condition ${ }^{\circ} k_{k}=F_{k}(0)$ for any $k \geq 1$, and by Theorem 1.1 the relations $\mathbb{S}_{r}=\stackrel{\circ}{\mathbb{S}}_{r}$ hold. Let us choose a solution $\stackrel{\circ}{Y}^{(0)}(z)$ close to $z=0$ in the Birkhoff-Levelt normal form, and define the corresponding central connection matrix $\dot{C}^{(0)}$ such that

$$
\stackrel{\circ}{Y}_{1}(z)=\stackrel{\circ}{Y}^{(0)}(z) \stackrel{\circ}{C}^{(0)} \text {. }
$$

\footnotetext{
${ }^{9}$ If the vanishing condition (1.20) fails, formal solutions are more complicated (see Theorem 4.1).
} 
We will prove that the class of formal solutions (1.23) reduces to only one element (thus the formal solution is unique) if and only if the diagonal entries of $\widehat{A}_{1}(0)$ do not differ by non-zero integers. This fact implies the following

Corollary 1.1. Let the assumptions of Theorem 1.1 hold. If the diagonal entries of $\widehat{A}_{1}(0)$ do not differ by non-zero integers, then there is a unique formal solution (1.23) of the system (1.21), whose coefficients necessarily satisfy the condition

$$
\stackrel{\circ}{F}_{k} \equiv F_{k}(0) .
$$

Hence, (1.21) only has at $z=\infty$ canonical fundamental solutions $\stackrel{\circ}{Y}_{1}(z), \stackrel{\circ}{Y}_{2}(z), \stackrel{\circ}{Y}_{3}(z)$, which coincide with the canonical solutions $Y_{1}(z, t), Y_{2}(z, t), Y_{3}(z, t)$ of (1.9) evaluated at $t=0$, namely:

$$
Y_{1}(z, 0)=\stackrel{\circ}{Y}_{1}(z), \quad Y_{2}(z, 0)=\stackrel{\circ}{Y}_{2}(z), \quad Y_{3}(z, 0)=\stackrel{\circ}{Y}_{3}(z)
$$

Moreover, for any $\stackrel{\circ}{Y}^{(0)}(z)$ there exists $Y^{(0)}(z, t)$ such that $Y^{(0)}(z, 0)=\stackrel{\circ}{Y}^{(0)}(z)$. The following equalities hold:

$$
\mathbb{S}_{1}=\stackrel{\mathscr{S}}{1}_{1}, \quad \mathbb{S}_{2}=\stackrel{\mathscr{S}}{2}_{2}, \quad C^{(0)}=\check{C}^{(0)} .
$$

Corollary 1.1 has a practical computational importance: the constant monodromy data (1.12) of the system (1.9) on the whole $\mathcal{U}_{\epsilon_{0}}(0)$ are computable just by considering the system (1.21) at the coalescence point $t=0$. This is useful for applications in the following two cases.

a) When $\widehat{A}_{1}(t)$ is known in a whole neighbourhood of a coalescence point, but the computation of monodromy data, which is highly transcendental, can be explicitly done (only) at a coalescence point, where (1.9) simplifies due to (1.20). An example is given in Section 22 for the sixth Painlevé equation. Another example will be given in [15] for the $A_{3}$-Frobenius manifold.

b) When $\widehat{A}_{1}(t)$ is explicitly known only at a coalescence point. This may happen in the case of Frobenius manifolds. So far, the theory of semisimple Frobenius manifolds has never been extended to semisimple coalescence points, which appear frequently in important cases, such as for example the quantum cohomology of Grassmannians [14], [15]. Our result is at the basis of the extension of the theory, as it will be thoroughly exposed in [15]. Theorem 1.1 and Corollary 1.1 allows the computation of local moduli (monodromy data) of a semisimple Frobenius manifold just by considering a coalescence point. The link between the present paper and [15] will be established in Section 21.

In the present paper, we also prove Theorem 1.2 below, which is the converse of Theorem 1.1. Assume that the system is isomonodromic on a simply connected domain $\mathcal{V} \subset \mathcal{U}_{\epsilon_{0}}(0)$ as in Definition 1.1. Note that now we are not assuming that $\widehat{A}_{1}(t)$ is holomorphic in the whole $\mathcal{U}_{\epsilon_{0}}(0)$, contrary to what has been done so far. As a result of [55], the fundamental solutions $Y_{r}(z, t), r=1,2,3$, and $A_{1}(t)$ can be analytically continued as meromorphic matrix valued functions on the universal covering of $\mathcal{U}_{\epsilon_{0}}(0) \backslash \Delta$, with movable poles at the Malgrange divisor [57] [50] [51] [52]. The coalescence locus $\Delta$ is in general a fixed branching locus. Moreover, although for $t \in \mathcal{V}$ the fundamental solutions $Y_{r}(z, t)$ have in $\mathcal{S}_{r}(\overline{\mathcal{V}})$ the canonical asymptotic behavior $Y_{F}(z, t)$ as in (1.6), in general this is no longer true when $t$ moves sufficiently far away from $\mathcal{V}$.

Nevertheless, if the vanishing condition (1.22) on Stokes matrices holds, then we can prove that the fundamental solutions $Y_{r}(z, t)$ and $\widehat{A}_{1}(t)$ have single-valued meromorphic continuation on $\mathcal{U}_{\epsilon_{0}}(0) \backslash \Delta$, so that $\Delta$ is not a branching locus. Moreover, the asymptotic behaviour is preserved, according to the following

Theorem 1.2. Let $\epsilon_{0}$ be as in (1.18). Consider the system (1.9). Let the matrix $\widehat{A}_{1}(t)$ be holomorphic on an open simply connected domain $\mathcal{V} \subset \mathcal{U}_{\epsilon_{0}}(0)$ such that the deformation is admissible and isomonodromic as in Definitions 1.1 and 1.2. Assume that the entries of the constant Stokes matrices satisfy the vanishing condition

$$
\left(\mathbb{S}_{1}\right)_{a b}=\left(\mathbb{S}_{1}\right)_{b a}=\left(\mathbb{S}_{2}\right)_{a b}=\left(\mathbb{S}_{2}\right)_{b a}=0 \text { whenever } u_{a}(0)=u_{b}(0), 1 \leq a \neq b \leq n .
$$


Then, as functions of $t$, the fundamental solutions $Y_{r}(z, t)$ and $\widehat{A}_{1}(t)$ admit single-valued meromorphic continuation on $\mathcal{U}_{\epsilon_{0}}(0) \backslash \Delta$. Moreover, for any $t \in \mathcal{U}_{\epsilon_{0}}(0) \backslash \Delta$ which is not a pole of $Y_{r}(z, \tilde{t})$ (i.e. which is not a point of the Malgrange divisor), we have

$$
Y_{r}(z, t) \sim Y_{F}(z, t) \text { for } z \rightarrow \infty \text { in } \widehat{\mathcal{S}}_{r}(t), \quad r=1,2,3,
$$

and

$$
Y_{r+1}(z, t)=Y_{r}(z, t) \mathbb{S}_{r}, \quad r=1,2 .
$$

The $\widehat{\mathcal{S}}_{r}(t)$ 's are the wide sectors described after the inequality (1.18) above.

We compare our results with the existing literature on isomonodromic deformations. The case when $\Delta$ is empty and $\widehat{A}_{1}(t)$ is any matrix does not add additional difficulties to the theory developed in [44]. Indeed, in the definition of isomonoromic deformations given above, not only we require that the monodromy matrix at $z=0$ is independent of $t$, but also the monodromy exponents $J^{(0)}, R^{(0)}$ and the connection matrix $C^{(0)}$ in (1.12) are constant (this is an isoprincipal deformation, in the language of [42]). Given these conditions on the exponents, and assuming that $\Delta=\emptyset$, one can essentially repeat the proofs given in [44]. For example, the case when $\Delta$ is empty and $\widehat{A}_{1}(t)$ is skew-symmetric and diagonalisable has been studied in [17], [19]. We also recall that in case of Fuchsian singularities only, isomonodromic deformations were completely studied ${ }^{10}$ in [11] and [42].

Isomonodromy deformations at irregular singular points with leading matrix admitting a Jordan form independent of $t$ were studied in [6] (with some minor Lidskii generic conditions). For example, if the singularity is at $z=\infty$ as in (1.5), the results of [6] apply to $\widehat{A}(z, t)=z^{k-1}\left(J+\sum_{j=1}^{\infty} \widehat{A}_{j}(t) z^{-j}\right)$, with Jordan form $J$ and Poincaré rank $k \geq 1$. Although the eigenvalues of $J$ have in general algebraic multiplicity greater than $1, J$ is "rigid", namely $u_{1}, \ldots, u_{n}$ do not depend on $t$.

Other investigations of isomonodromy deformations at irregular singularities can be found in [27] and [7]. Nevertheless, these results do not apply to our coalescence problem. For example, the third admissibility conditions of definition 10 of [7] is not satisfied in our case. In [27] the system with $A(z, t)=z^{r-1} B(z, t), r \in \mathbb{Q}$, is considered, such that $B(\infty, t)$ has distinct eigenvalues; $z=\infty$ satisfying this condition is called a simple irregular singular point. This simplicity condition does not apply in our case.

The results of [46], cited above, are applied in [47] to the $3 \times 3$ isomonodromic description of the Painlevé 6 equation and its coalescence to Painlevé 5 . In this case, the limiting system for $t \rightarrow 0$ has leading matrix with a $2 \times 2$ Jordan block, so that the fundamental matrices $Y_{r}(z, t)$ diverge.

Isomonodromic deformations of a system such as our (1.9) (with $z \mapsto 1 / z, \widehat{A}_{0} \mapsto Z, \widehat{A}_{1} \mapsto f$ ) appears also in [12]. Nevertheless, the deformations in Section 3 of [12] are of a very particular kind. Indeed, the eigenvalues $u_{1}, \ldots, u_{n}$ of the matrix $Z$ in [12], which is the analogue of our $\widehat{A}_{0}$, are deformation parameters, but always satisfy the condition

$$
\begin{aligned}
u_{1} & =\cdots=u_{p_{1}}, \\
u_{p_{1}+1} & =\cdots=u_{p_{1}+p_{2}}, \\
\cdots & \\
u_{p_{1}+\cdots+p_{s-1}+1} & =\cdots=u_{p_{1}+\cdots+p_{s}},
\end{aligned}
$$

with $p_{1}+\cdots+p_{s}=n$. Thus, no splitting of coalescences occurs, so that the deformations are always inside the same "stratum" of the coalescence locus. Moreover, the matrix $f=f(Z)$ in [12], which is the analogue of our $\widehat{A}_{1}$, satisfies quite restrictively requirements that the diagonal is zero and $\left(\widehat{A}_{1}\right)_{a b}=0$ whenever $u_{a}=u_{b}, 1 \leq a \neq b \leq n$. These conditions are always satisfied along the deformation "stratum" of [12]; they are a particular case or the more general conditions of Proposition 4.2 in our paper below. For these reasons, an adaptation of the classical Jimbo-Miwa-Ueno results [44] (and those of [8] for a connection on a G-bundle, with G a complex and reductive group) can be done verbatim, in order to

\footnotetext{
${ }^{10}$ In [11] it is only assumed that the monodromy matrices are constant. This generates non-Schlesinger deformations. On the other hand, an isopricipal deformation always leads to Schlesinger deformations [42].
} 
describe the isomonodromicity condition for such a very particular kind of deformations. In the present paper, we studied general isomonodromic deformations of the system (1.9), not necessarily the simple decomposition of the spectrum as in (1.24)-(1.27).

\subsection{Plan of the Paper.}

- In Part I, we study formal and fundamental solutions of the system (1.1) as $z \rightarrow \infty$, both at coalescence points and away from them. We give necessary and sufficient conditions for a formal solution, computed away from coalescence points, to admit holomorphic continuation to the coalescence locus (see Proposition 5.1).

- In Part II, we study the Stokes phenomenon at $z=\infty$ for the system (1.1), both at coalescence and non-coalescence points. We show existence and uniqueness results at coalescence points.

- In Part III, under Assumption 1 we discuss the analytic continuation of fundamental solutions of (1.1). We show that $\mathcal{U}_{\epsilon_{0}}(0)$ splits into topological cells, determined by the fact that Stokes rays associated with $\Lambda(t)$ cross a fixed admissible ray. In Theorem 14.1 and Corollary 14.1 we give sufficient conditions such that fundamental solutions can be analytically continued to the whole $\mathcal{U}_{\epsilon_{0}}(0)$, preserving their asymptotic representation, so that the Stokes matrices admit the limits (1.14). Notice that for the results in Parts I-III no isomonodromicity is required.

- In Part IV, we formulate the monodromy preserving deformation theory for system (1.9). We prove Theorem 1.1, Corollary 1.1 and Theorem 1.2.

- In Part V, we show how Theorem 1.1 and Corollary 1.1 can be applied to Frobenius Manifolds and to the sixth Painlevé equation.

Remark 1.2. In the main body of the paper, the matrices $Y_{r}$, sectors $\mathcal{S}_{r}$ and Stokes matrices $\mathbb{S}_{r}$ will be labelled differently as $Y_{\nu+(r-1) \mu}, \mathcal{S}_{\nu+(r-1) \mu}$ and $\mathbb{S}_{\nu+(r-1) \mu}, \nu, \mu \in \mathbb{Z}$. This labelling will be explained.

Acknowledgements: We thank Marco Bertola for helpful discussions concerning the proof of Theorem 1.2. D. Guzzetti remembers with gratitude Andrei Kapaev for insightful discussions at the time when this work was initiated.

\section{PART I: Structure of Fundamental Solutions}

Notational Remarks: If $\alpha<\beta$ are real numbers, an open sector and a closed sector with central opening angle $\beta-\alpha>0$ are respectively denoted by

$$
S(\alpha, \beta):=\{z \in \mathcal{R} \mid \alpha<\arg z<\beta\}, \quad \bar{S}(\alpha, \beta):=\{z \in \mathcal{R} \mid \alpha \leq \arg z \leq \beta\} .
$$

The rays with directions $\alpha$ and $\beta$ will be called the right and left boundary rays respectively. If $\bar{S}\left(\theta_{1}, \theta_{2}\right) \subset S(\alpha, \beta)$, then $\bar{S}\left(\theta_{1}, \theta_{2}\right)$ is called a proper (closed) subsector.

Given a function $f(z)$ holomorphic on a sector containing $\bar{S}(\alpha, \beta)$, we say that it admits an asymptotic expansion $f(z) \sim \sum_{k=0}^{\infty} a_{k} z^{-k}$ for $z \rightarrow \infty$ in $\bar{S}(\alpha, \beta)$, if for any $m \geq 0, \lim _{z \rightarrow \infty} z^{m}\left(f(z)-\sum_{k=0}^{m} a_{k} z^{-k}\right)=0$, $z \in \bar{S}(\alpha, \beta)$. If $f$ depends on parameters $t$, the asymptotic representation $f(z, t) \sim \sum_{k=0}^{\infty} a_{k}(t) z^{-k}$ is said to be uniform in $t$ belonging to a compact subset $K \subset \mathbb{C}^{m}$, if the limits above are uniform in $K$. In case the sector is open, we write $f(z) \sim \sum_{k=0}^{\infty} a_{k} z^{-k}$ as $z \rightarrow \infty$ in $S(\alpha, \beta)$ if the limits above are zero in every proper closed subsector of $S(\alpha, \beta)$. When we take the limits above for matrix valued functions $A=\left(A_{i j}(z, t)\right)_{i, j=1}^{n}$, we use the $\operatorname{norm}|A|:=\max _{i j}\left|A_{i j}\right|$.

\section{Deformation of a Differential System with Singularity of the Second Kind}

We consider system (1.1) of the Introduction, namely

$$
\frac{d Y}{d z}=A(z, t) Y, \quad t=\left(t_{1}, t_{2}, \ldots, t_{m}\right) \in \mathbb{C}^{m},
$$


depending on $m$ complex parameters ${ }^{11} t$. The $n \times n$ matrix $A(z, t)$ is holomorphic in $(z, t)$ for $|z| \geq N_{0}>0$ and $|t| \leq \epsilon_{0}$, for some positive constants $N_{0}$ and $\epsilon_{0}$, with uniformly convergent Taylor expansion

$$
A(z, t)=\sum_{j=0}^{\infty} A_{j}(t) z^{-j} .
$$

The coefficients $A_{j}(t)$ are holomorphic for $|t| \leq \epsilon_{0}$. We assume that $A_{0}(0)$ is diagonalisable, with distinct eigenvalues $\lambda_{1}, \ldots, \lambda_{s}, s \leq n$. We are interested in the case when $s$ is strictly less than $n$. Up to a constant gauge transformation, there is no loss of generality in assuming that

$$
A_{0}(0)=\Lambda:=\Lambda_{1} \oplus \cdots \oplus \Lambda_{s}, \quad \Lambda_{i}:=\lambda_{i} I_{p_{i}}, \quad i=1,2, \ldots, s \leq n,
$$

being $I_{p_{i}}$ the $p_{i} \times p_{i}$ identity matrix. If $A_{0}(t)$ is holomorphically similar to $\Lambda(t)$, as in (1.3), then $\Lambda=\Lambda(0)$. However, at this stage of the discussion we do not assume holomorphic similarity, so we keep the notation $\Lambda$ instead of $\Lambda(0)$.

Remark 2.1. A result due to Kostov [48] states that, if system (2.1) is such that $A(z, 0)=A_{0}(0)+$ $A_{1}(0) / z$, and if the matrix $A_{1}(0)$ has no eigenvalues differing by a non-zero integers, than there exists a gauge transformation $Y=W(z, t) \widetilde{Y}$, with $W(z, t)$ holomorphic at $z=\infty$ and $t=0$, such that (2.1) becomes a system like (1.8):

$$
\frac{d \widetilde{Y}}{d z}=\left(\widetilde{A}_{0}(t)+\frac{\widetilde{A}_{1}(t)}{z}\right) \widetilde{Y}
$$

Nevertheless, since $A_{0}(0)$ has non-distinct eigenvalues, we cannot find in general a gauge transformation holomorphic at $z=\infty$ which transforms $A(z, 0)$ of the system $(2.1)$ into $A_{0}(0)+A_{1}(0) / z$ (see also [9] and references therein). Therefore the system (2.1) - namely the system (1.1) - is more general than system (2.4), namely than (1.8).

2.1. Sibuya's Theorem. General facts about eigenvalues and eigenvectors of a matrix $M(t)$, depending holomorphically on $t$ in a domain $\mathcal{D} \subset \mathbb{C}^{m}$, such that $M(0)$ has eigenvalues $\lambda_{1}, \ldots, \lambda_{s}, s \leq n$, can be found in [49] and at page 63-87 of [45]. If $s$ is strictly smaller than $n$, then $t=0$ is a coalescence point. For $\mathcal{D} \subset \mathbb{C}^{m}$ and $m=1$ the coalescence points are isolated, while for $m \geq 2$ they form the coalescence locus. Except for the special case when $M(t)$ is holomorphically similar to a Jordan form $J(t)$, which means that there exists an invertible holomorphic matrix $G_{0}(t)$ on $\mathcal{D}$ such that $\left(G_{0}(t)\right)^{-1} M(t) G_{0}(t)=J(t)$, in general the eigenvectors of $M(t)$ are holomorphic in the neighborhood of a non-coalescence point, but their analytic continuation is singular at the coalescence locus. For example,

$$
M(t)=\left(\begin{array}{ll}
0 & 1 \\
t & 0
\end{array}\right), \quad t \in \mathbb{C}
$$

has eigenvalues $\lambda_{ \pm}= \pm \sqrt{t}$, which are branches of $f(t)=t^{1 / 2}$, with ramification at $\Delta=\{t=0\}$. The eigenvectors can be chosen to be either

$$
\vec{\xi}_{ \pm}=( \pm 1 / \sqrt{t}, 1), \text { or } \vec{\xi}_{ \pm}=( \pm 1, \sqrt{t}) .
$$

The matrix $G_{0}(t):=\left[\vec{\xi}_{+}(t), \vec{\xi}_{-}(t)\right]$ puts $M(t)$ in diagonal form $G_{0}(t)^{-1} A_{0}(t) G_{0}(t)=\operatorname{diag}(\sqrt{t},-\sqrt{t})$, for $t \neq 0$, while $M(0)$ is in Jordan non-diagonal form. Either $G_{0}(t)$ or $G_{0}(t)^{-1}$ is singular at $t=0$. The branching could be eliminated by changing deformation parameter to $s=t^{1 / 2}$. Nevertheless, this would not cure the singularity of $G_{0}$ or $G_{0}^{-1}$ at $s=0$. Another example is

$$
M(t)=\left(\begin{array}{ll}
1 & t \\
0 & 1
\end{array}\right), \quad t \in \mathbb{C} .
$$

The eigenvalues $u_{1}=u_{2}=1$ are always coalescing. The Jordan types at $t \neq 0$ and $t=0$ are different. Indeed, $M(0)=\operatorname{diag}(1,1)$, while for $t \neq 0$,

$$
G_{0}(t)^{-1} M(t) G_{0}(t)=\left(\begin{array}{cc}
1 & 1 \\
0 & 1
\end{array}\right), \quad G_{0}(t):=\left(\begin{array}{cc}
t & 0 \\
0 & 1
\end{array}\right) .
$$

Now, $G_{0}(t)$ is not invertible and $G_{0}(t)^{-1}$ diverges at $t=0$.

\footnotetext{
${ }^{11}$ Later, we will take $n=m$, as in (1.15).
} 
In the above examples, the Jordan type of $M(t)$ changes. In the next example, the Jordan form remains diagonal, and nevertheless $G_{0}(t)$ is singular. Consider

$$
M(t)=\left(\begin{array}{cc}
1+t_{1} & t_{2} \\
0 & 1-t_{2}
\end{array}\right), \quad t=\left(t_{1}, t_{2}\right) \in \mathbb{C}^{2}
$$

The eigenvalues coalesce at $t=0$, where $M(0)=I$. Moreover, there exists a diagonalizing matrix $G_{0}(t)$ such that

$$
G_{0}(t)^{-1} M(t) G_{0}(t)=\left(\begin{array}{cc}
1+t_{1} & 0 \\
0 & 1-t_{2}
\end{array}\right) \text { is diagonal, } \quad G_{0}(t)=\left(\begin{array}{cc}
a(t) & -t_{2} b(t) \\
0 & \left(t_{1}+t_{2}\right) b(t)
\end{array}\right),
$$

for arbitrary non-vanishing holomorphic functions $a(t), b(t)$. At $t=0$ the matrix $G_{0}(t)$ has zero determinant and $G_{0}(t)^{-1}$ diverges.

Although $M(t)$ is not in general holomorphically similar to a Jordan form, holomorphic similarity can always be realised between $M(t)$ and a block-diagonal matrix $\widehat{M}(t)$ having the same block structure of a Jordan form of $M(0)$, as follows.

Lemma 2.1. [LEMMA 1 of [66]]: Let $M(t)$ be a $n \times n$ matrix holomorphically depending on $t \in \mathbb{C}^{m}$, with $|t| \leq \epsilon_{0}$, where $\epsilon_{0}$ is a positive constant. Let $\lambda_{1}, \lambda_{2}, \ldots, \lambda_{s}$ be the distinct eigenvalues of $M(0)$, with multiplicities $p_{1}, p_{2}, \ldots, p_{s}$, so that $p_{1}+p_{2}+\cdots+p_{s}=n$. Assume that $M(0)$ is in Jordan form

$$
M(0)=M_{1}(0) \oplus \cdots \oplus M_{s}(0)
$$

where

$$
M_{j}(0)=\lambda_{j} I_{p_{j}}+\mathcal{H}_{j}, \quad \mathcal{H}_{j}=\left[\begin{array}{ccccc}
0 & \mathfrak{h}_{j 1} & & & \\
& 0 & \mathfrak{h}_{j 2} & & \\
& & \ddots & \ddots & \\
& & & 0 & \mathfrak{h}_{j p_{j}-1} \\
& & & & 0
\end{array}\right], \quad 1 \leq j \leq s
$$

$\mathfrak{h}_{j k}$ being equal to 1 or 0 . Then, for sufficiently small $0<\epsilon \leq \epsilon_{0}$ there exists a matrix $G_{0}(t)$, holomorphic in $t$ for $|t| \leq \epsilon$, such that

$$
G_{0}(0)=I
$$

and $\widehat{M}(t)=\left(G_{0}(t)\right)^{-1} M(t) G_{0}(t)$ has block diagonal form

$$
\widehat{M}(t)=\widehat{M}_{1}(t) \oplus \cdots \oplus \widehat{M}_{s}(t),
$$

where $\widehat{M}_{j}(t)$ are $p_{j} \times p_{j}$ matrices. For $|t| \leq \epsilon, \widehat{M}_{i}(t)$ and $\widehat{M}_{j}(t)$ have no common eigenvalues for any $i \neq j$.

Remark 2.2. The lemma also holds when $t \in \mathbb{R}^{m}$ in the continuous (not necessarily holomorphic) setting.

Lemma 2.1 can be applied to $M(t) \equiv A_{0}(t)$ in $(2.2)$, with $A_{0}(0)=\Lambda$. Therefore ${ }^{12}$

$$
\begin{gathered}
\widehat{A}_{0}(t):=G_{0}(t)^{-1} A_{0}(t) G_{0}(t)=\widehat{A}_{11}^{(0)}(t) \oplus \cdots \oplus \widehat{A}_{s s}^{(0)}(t), \\
G_{0}(0)=I, \quad \widehat{A}_{0}(0)=A_{0}(0)=\Lambda .
\end{gathered}
$$

Remark 2.3. $G_{0}(t)$ is determined up to $G_{0} \mapsto G_{0}(t) \Delta_{0}(t)$, where $\Delta_{0}(t)$ is any block-diagonal matrix solution of $\left[\Delta_{0}(t), \widehat{A}_{0}(t)\right]=0$. Sibuya's normalization condition $G_{0}(0)=I$ can be softened to $G_{0}(0)=$ $\Delta_{0}$

We define a family of sectors $\mathcal{S}_{\nu}$ in $\mathcal{R}$ and state Sibuya's theorem. Let $\arg _{p}\left(\lambda_{j}-\lambda_{k}\right)$ be the principal determination. Let $\eta \in \mathbb{R}$ be an admissible direction for $\Lambda$ in the $\lambda$-plane (we borrow this name and the following definition of the $\eta_{\nu}$ 's and $\tau_{\nu}$ 's from [2] and [5]). By definition, this means that,

$$
\eta \neq \arg _{p}\left(\lambda_{j}-\lambda_{k}\right) \bmod (2 \pi), \quad \forall 1 \leq j \neq k \leq s .
$$

\footnotetext{
${ }^{12}$ Given a $n \times n$ matrix $A_{0}$, partitioned into $s^{2}$ blocks $(s \leq n)$, we use the notation $A_{i j}^{(0)}, 1 \leq i, j \leq s$, to denote the block in position $(i, j)$. Such a block has dimension $p_{i} \times p_{j}$, with $p_{1}+\ldots+p_{n}=n$.
} 
Introduce another determination $\widehat{\arg }$ as follows:

$$
\eta-2 \pi<\widehat{\arg }\left(\lambda_{j}-\lambda_{k}\right)<\eta, \quad 1 \leq j \neq k \leq s .
$$

Let $2 \mu, \mu \in \mathbb{N}$, be the number of values $\widehat{\arg }\left(\lambda_{j}-\lambda_{k}\right)$, when $(j, k)$ spans all the indices $1 \leq j \neq k \leq s$. $^{13}$ Denote the $2 \mu$ values of $\widehat{\arg }\left(\lambda_{j}-\lambda_{k}\right)$ with $\eta_{0}, \eta_{1}, \ldots, \eta_{2 \mu-1}$, according to the following ordering:

$$
\eta>\eta_{0}>\cdots>\eta_{\mu-1}>\eta_{\mu}>\cdots>\eta_{2 \mu-1}>\eta-2 \pi
$$

Clearly

$$
\eta_{\nu+\mu}=\eta_{\nu}-\pi, \quad \nu=0,1, \ldots, \mu-1 .
$$

Consider the following directional angles in the $z$-plane

$$
\tau:=\frac{3 \pi}{2}-\eta, \quad \tau_{\nu}:=\frac{3 \pi}{2}-\eta_{\nu}, \quad 0 \leq \nu \leq 2 \mu-1 .
$$

From (2.8) if follows that,

$$
\tau<\tau_{0}<\cdots<\tau_{\mu-1}<\tau_{\mu}<\cdots<\tau_{2 \mu-1}<\tau+2 \pi .
$$

From (2.9) if follows that,

$$
\tau_{\nu+\mu}=\tau_{\nu}+\pi, \quad \nu=0,1, \ldots, \mu-1 .
$$

The extension of the above to directions in $\mathcal{R}$ is obtained by the following definition:

$$
\tau_{\nu+k \mu}:=\tau_{\nu}+k \pi, \quad k \in \mathbb{Z} .
$$

This allows to speak of directions $\tau_{\nu}$ for any $\nu \in \mathbb{Z}$.

Definition 2.1 (Sector $\mathcal{S}_{\nu}$ ). We define the following sectors of central opening angle greater than $\pi$ :

$$
\mathcal{S}_{\nu}:=S\left(\tau_{\nu}-\pi, \tau_{\nu+1}\right) \equiv S\left(\tau_{\nu-\mu}, \tau_{\nu+1}\right), \quad \nu \in \mathbb{Z}
$$

Theorem 2.1 (Sibuya [66] [37]). Let $A(z, t)$ be holomorphic in $(z, t)$ for $|z| \geq N_{0}>0$ and $|t| \leq \epsilon_{0}$ as in (2.2), such that $A_{0}(0)=\Lambda=\Lambda_{1} \oplus \cdots \oplus \Lambda_{s}$, as in (2.3). Pick up a sector $\mathcal{S}_{\nu}$ as in (2.12). Then, for any proper closed subsector $\bar{S}(\alpha, \beta)=\left\{z \mid \tau_{\nu}-\pi<\alpha \leq \arg z \leq \beta<\tau_{\nu+1}\right\} \subset \mathcal{S}_{\nu}$, there exist a sufficiently large positive number $N \geq N_{0}$, a sufficiently small positive number $\epsilon \leq \epsilon_{0}$, and matrices $G_{0}(t)$ and $G(z, t)$ with the following properties:

i) $G_{0}(t)$ is holomorphic for $|t| \leq \epsilon$ and

$$
G_{0}(0)=I, \quad \widehat{A}_{0}(t):=G_{0}(t)^{-1} A_{0}(t) G_{0}(t) \text { is block-diagonal as in (2.6). }
$$

ii) $G(z, t)$ is holomorphic in $(z, t)$ for $|z| \geq N, z \in \bar{S}(\alpha, \beta),|t| \leq \epsilon$;

iii) $G(z, t)$ has a uniform asymptotic expansion for $|t| \leq \epsilon$, with holomorphic coefficients $G_{k}(t)$ :

$$
G(z, t) \sim I+\sum_{k=1}^{\infty} G_{k}(t) z^{-k}, \quad z \rightarrow \infty, \quad z \in \bar{S}(\alpha, \beta),
$$

iv) The gauge transformation

$$
Y(z, t)=G_{0}(t) G(z, t) \tilde{Y}(z, t),
$$

reduces the initial system to a block diagonal form

$$
\frac{d \tilde{Y}}{d z}=B(z, t) \tilde{Y}, \quad B(z, t)=B_{1}(z, t) \oplus \cdots \oplus B_{s}(z, t),
$$

where $B(z, t)$ is holomorphic in $(z, t)$ in the domain $|z| \geq N, z \in \bar{S}(\alpha, \beta),|t| \leq \epsilon$,

and has a uniform asymptotic expansion for $|t| \leq \epsilon$, with holomorphic coefficients $B_{k}(t)$,

$$
B(z, t) \sim \widehat{A}_{0}(t)+\sum_{k=1}^{\infty} B_{k}(t) z^{-k}, \quad z \rightarrow \infty, \quad z \in \bar{S}(\alpha, \beta) .
$$

In particular, setting $\widehat{A}_{1}(t):=G_{0}^{-1}(t) A_{1}(t) G_{0}(t)$, then $B_{1}(t)=\widehat{A}_{11}^{(1)}(t) \oplus \cdots \oplus \widehat{A}_{s s}^{(1)}(t)$.

\footnotetext{
$132 \mu \leq s(s-1)$, with "=" occurring when $\arg \left(\lambda_{j}-\lambda_{k}\right) \neq \arg \left(\lambda_{r}-\lambda_{s}\right) \bmod 2 \pi$ for any $(j, k) \neq(r, s)$.
} 
Remark 2.4. In the theorem above, $\epsilon$ is such that $\widehat{A}_{i i}^{(0)}(t)$ and $\widehat{A}_{j j}^{(0)}(t)$ have no common eigenvalues for any $i \neq j$ and $|t| \leq \epsilon$. Observe that one can always choose $\beta-\alpha>\pi$.

Remark 2.5. $\mathcal{S}_{\nu}$ coincides with a sector $\left\{z \in \mathcal{R} \mid-3 \pi / 2-\omega_{-}<r \arg z<3 \pi / 2-\omega_{+}\right\}$, introduced by Sibuya in [37]. A closed subsector $\bar{S}(\alpha, \beta)$ is a sector $\mathcal{D}(N, \gamma)$ introduced by Sibuya in [66].

Remark 2.6. If $\Lambda=\lambda_{1} I$, Theorem 2.1 gives no new information, being $G_{0}(t)=G(z, t) \equiv I$ and $\mathcal{S}_{\nu}=\mathcal{R}$.

- A Short Review of the Proof: The $z$-constant gauge transformation $Y(z, t)=G_{0}(t) \widehat{Y}(z, t)$ transforms (2.1) into

$$
\frac{d \widehat{Y}}{d z}=\widehat{A}(z, t) \widehat{Y}, \quad \widehat{A}(z, t)=\sum_{i=0}^{\infty} \widehat{A}_{i}(t) z^{-i}, \quad \widehat{A}_{i}(t):=G_{0}^{-1}(t) A_{i}(t) G_{0}(t) .
$$

Another gauge transformation $\widehat{Y}(z, t)=G(z, t) \widetilde{Y}(z, t)$ yields (2.13). Substitution into (2.15) gives the differential equation

$$
G^{\prime}+G B=\widehat{A}(z, t) G
$$

with unknowns $G(z, t), B(z, t)$. If formal series $G(z, t)=I+\sum_{j=1}^{\infty} G_{j}(t) z^{-j}$ and $B(z, t)=\widehat{A}_{0}(t)+$ $\sum_{j=1}^{\infty} B_{j}(t) z^{-j}$ are inserted into (2.16), the following recursive equations ( $t$ is understood) are found:

For $l=0: B_{0}(t)=\widehat{A}_{0}(t)$.

For $l=1$ :

$$
\widehat{A}_{0} G_{1}-G_{1} \widehat{A}_{0}=-\widehat{A}_{1}+B_{1}
$$

$\underline{\text { For } l \geq 2}$

$$
\widehat{A}_{0} G_{l}-G_{l} \widehat{A}_{0}=\left[\sum_{j=1}^{l-1}\left(G_{j} B_{l-j}-\widehat{A}_{l-j} G_{j}\right)-\widehat{A}_{l}\right]-(l-1) G_{l-1}+B_{l} .
$$

Once $G_{0}(t)$ has been fixed, the recursion equations can be solved. A solution $\left\{G_{l}(t)\right\}_{l=1}^{\infty},\left\{B_{l}(t)\right\}_{l=1}^{\infty}$ is not unique in general. The following choice is possible:

$$
G_{j j}^{(l)}(t)=0, \quad 1 \leq j \leq s, \quad \text { [diagonal blocks are zero], }
$$

and

$$
B_{l}(t)=B_{1}^{(l)}(t) \oplus \cdots \oplus B_{s}^{(l)}(t), \quad \text { [off-diagonal blocks are zero]. }
$$

Then, the $G_{l}(t)$ 's and $B_{l}(t)$ 's are determined by the recursion relations, because for a diagonal block $[j, j]$ the l.h.s of $(2.17)$ and $(2.18)$ is equal to 0 and the r.h.s determines the only unknown variable $B_{j j}^{(l)}$. For off-diagonal blocks $[i, j]$ there is no unknown in the r.h.s while in the l.h.s the following expression appears

$$
\widehat{A}_{i i}^{(0)}(t) G_{i j}^{(l)}-G_{i j}^{(l)} \widehat{A}_{j j}^{(0)}(t), \quad 1 \leq i \neq j \leq s .
$$

For $|t| \leq \epsilon$ small enough, $\widehat{A}_{i i}^{(0)}(t)$ and $\widehat{A}_{j j}^{(0)}(t)$ have no common eigenvalues, so the equation is solvable for $G_{i j}^{(l)}$. With the above choice, Sibuya [66] proves that there exist actual solutions $G(z, t)$ and $B(z, t)$ of (2.16) with asymptotic expansions $I+\sum_{j} G_{j}(t) z^{-j}$ and $\widehat{A}_{0}+\sum_{j} B_{j}(t) z^{-j}$ respectively. We remark that the proof relies on the above choice. It is evident that this choice also ensures that all the coefficients $G_{j}(t)$ 's and $B_{j}(t)$ 's are holomorphic where the $\widehat{A}_{j}(t)$ 's are. Note that $(2.17)$ yields $B_{1}(t)=\widehat{A}_{11}^{(1)} \oplus \cdots \oplus$ $\widehat{A}_{s s}^{(1)}(t)$.

\section{Fundamental Solutions of (2.13)}

The system (2.13) admits block-diagonal fundamental solutions $\tilde{Y}(z, t)=\tilde{Y}_{1}(z, t) \oplus \cdots \oplus \tilde{Y}_{s}(z, t)$. Here, $\tilde{Y}_{i}(z, t)$ is a $p_{i} \times p_{i}$ fundamental matrix of the $i$-th diagonal block of (2.13). The problem is reduced to solving a system whose leading matrix has only one eigenvalue. The case when $A_{0}(t)$ has distinct eigenvalues for $|t|$ small is well known (see [37], and also [2] for the $t$-independent case). The case when $A_{0}(0)=\Lambda$ is diagonalisable, with $s \leq n$ distinct eigenvalues, will be studied here and in the subsequent 
sections.

We do another gauge transformation

$$
\widetilde{Y}(z, t)=e^{\Lambda z} Y_{\text {red }}(z, t),
$$

where the subscript red stand for "rank reduced". We substitute into (2.13) and find

$$
\ell^{\prime z}\left(\Lambda Y_{\text {red }}+Y_{\text {red }}^{\prime}\right)=B(z, t) \ell^{\not / z} Y_{\text {red }}
$$

The exponentials cancel because $B(z, t)$ is block diagonal with the same structure as $\Lambda$. Thus, we obtain

$$
\frac{d Y_{\text {red }}}{d z}=\frac{1}{z} B_{\text {red }}(z, t) Y_{\text {red }},
$$

with

$$
\begin{aligned}
& B_{r e d}(z, t):=z(B(z, t)-\Lambda)=B_{1}^{(r e d)}(z, t) \oplus \cdots \oplus B_{s}^{(r e d)}(z, t), \\
& B_{r e d}(z, t) \sim z\left(\widehat{A}_{0}(t)-\Lambda\right)+\sum_{k=1}^{\infty} B_{k}(t) z^{-k+1} .
\end{aligned}
$$

Fundamental solutions can be taken with block diagonal structure,

$$
Y_{\text {red }}(z, t)=Y_{1}^{(r e d)}(z, t) \oplus \cdots \oplus Y_{s}^{(r e d)}(z, t) .
$$

where $Y_{i}^{(r e d)}(z, t)$ solves

$$
\frac{d Y_{i}^{(r e d)}}{d z}=\frac{1}{z} B_{i}^{(r e d)}(z, t) Y_{i}^{(r e d)} .
$$

The exponential $e^{\Lambda z}$ commutes with the above matrices, hence a fundamental solution of (2.1) exists in the form

$$
Y(z, t)=G_{0}(t) G(z, t) Y_{\text {red }}(z, t) e^{\Lambda z} .
$$

We proceed as follows. In Section 4 we describe the structure of fundamental solutions of (2.1) for $t=0$ fixed. In Section 5 we describe the structure of fundamental solutions at other points $t \in \mathcal{U}_{\epsilon_{0}}(0)$.

\section{A Fundamental Solution of (2.1) at $t=0$}

At $t=0$, the rank is reduced, since the system (3.2) becomes a Fuchsian system in $\bar{S}(\alpha, \beta)$,

$$
\frac{d Y_{\text {red }}}{d z}=\frac{1}{z} B_{\text {red }}(z, 0) Y_{\text {red }}
$$

with $B_{\text {red }}(z, 0) \sim \sum_{k=1}^{\infty} B_{k}(0) z^{-k+1}$ for $z \rightarrow \infty$ in $\bar{S}(\alpha, \beta)$. Let $J_{i}$ be a Jordan form of the $i$-th block $B_{i}^{(1)}(0)=\widehat{A}_{i i}^{(1)}(0) \equiv A_{i i}^{(1)}(0), 1 \leq i \leq s$. Following [69], we choose $J_{i}$ arranged into $h_{i} \leq p_{i}$ Jordan blocks $J_{1}^{(i)}, \ldots, J_{h_{i}}^{(i)}$

$$
J_{i}=J_{1}^{(i)} \oplus \cdots \oplus J_{h_{i}}^{(i)}
$$

Each block $J_{j}^{(i)}, 1 \leq j \leq h_{i}$, has dimension $r_{j} \times r_{j}$, with $r_{j} \geq 1, r_{1}+\cdots+r_{h_{i}}=p_{i}$. Each $J_{j}^{(i)}$ has only one eigenvalue $\mu_{j}^{(i)}$, with structure,

$$
\begin{gathered}
J_{j}^{(i)}=\mu_{j}^{(i)} I_{r_{j}}+H_{r_{j}}, r_{j} \times r_{j} \text { identity matrix, } \\
H_{r_{j}}=0 \text { if } r_{j}=1, \quad H_{r_{j}}=\left[\begin{array}{ccccc}
0 & 1 & & & \\
& 0 & 1 & & \\
& & \ddots & \ddots & \\
& & & 0 & 1 \\
& & & 0
\end{array}\right] \quad \text { if } r_{j} \geq 2 .
\end{gathered}
$$


Note that $\mu_{1}^{(i)}, \ldots, \mu_{h_{i}}^{(i)}$ are not necessarily distinct. One can choose a $t$-independent matrix $\Delta_{0}=$ $\Delta_{1}^{(0)} \oplus \cdots \oplus \Delta_{s}^{(0)}$, in the block-diagonal of Remark 2.3, such that $\left(\Delta_{i}^{(0)}\right)^{-1} \widehat{A}_{i i}^{(1)}(0) \Delta_{i}^{(0)}=J_{i}$. Hence,

$$
\Delta_{0}^{-1} \widehat{A}_{1}(0) \Delta_{0} \equiv \Delta_{0}^{-1} A_{1}(0) \Delta_{0}=\left[\begin{array}{cccc}
J_{1} & * & * & * \\
* & J_{2} & & * \\
* & * & \ddots & * \\
* & * & * & J_{s}
\end{array}\right] \text {. }
$$

The transformation $Y_{\text {red }}=\Delta_{0} X_{\text {red }}$ of the system (4.1) yields ${ }^{14}$

$$
\begin{gathered}
\frac{d X_{\text {red }}}{d z}=\frac{1}{z} \mathcal{B}_{\text {red }}(z) X_{\text {red }}, \quad \mathcal{B}_{\text {red }}(z):=\Delta_{0}^{-1} B_{\text {red }}(z, 0) \Delta_{0} \\
\mathcal{B}_{\text {red }}(z) \sim J+\sum_{k=1}^{\infty} \frac{\mathcal{B}_{k+1}}{z^{k}}, \quad \mathcal{B}_{k}=\Delta_{0}^{-1} B_{k}(0) \Delta_{0} .
\end{gathered}
$$

The system (4.3) has block-diagonal fundamental solutions $X_{\text {red }}=X_{1}^{(\text {red })} \oplus \cdots \oplus X_{1}^{(r e d)}$, each block satisfying

$$
\frac{d X_{i}^{(r e d)}}{d z}=\frac{1}{z} \mathcal{B}_{i}^{(r e d)}(z) X_{i}^{(r e d)}, \quad 1 \leq i \leq s .
$$

Now, $J_{i}$ has the unique decomposition

$$
\begin{aligned}
& J_{i}=D_{i}+S_{i}, \quad D_{i}=\text { diagonal matrix of integers, } \\
& S_{i}=\text { Jordan form with diagonal elements of real part } \in[0,1) .
\end{aligned}
$$

For $i=1,2, \ldots, s$, let $m_{i} \geq 0$ be the maximum integer difference between couples of eigenvalues of $J_{i}$ $\left(m_{i}=0\right.$ if eigenvalues do not differ by integers). Let $\bar{m}:=\max _{i=1, . ., s} m_{i}$. The general theory of Fuchsian systems assures that (4.4) has a fundamental matrix solution

$$
X_{i}^{(r e d)}(z)=K_{i}(z) z^{D_{i}} z^{L_{i}}, \quad K_{i}(z) \sim I+\sum_{j=1}^{\infty} K_{j}^{(i)} z^{-j}, \quad z \rightarrow \infty \text { in } \bar{S}(\alpha, \beta) .
$$

Here $L_{i}:=S_{i}+R_{i}$, where the matrix $R_{i}$ is a sum $R_{i}=R_{(1), i}+\cdots R_{\left(m_{i}\right), i}$, whose terms satisfy

$$
\left[R_{(l), i}\right]_{\text {block } a, b} \neq 0 \quad \text { only if } \quad \mu_{b}^{(i)}-\mu_{a}^{(i)}=l>0 \text { integer. }
$$

Let

$$
D:=D_{1} \oplus \cdots \oplus D_{s}, \quad S:=S_{1} \oplus \cdots \oplus S_{s}, \quad R:=R_{1} \oplus \cdots \oplus R_{s}, \quad L:=R+S .
$$

Observe now that $R$ has a sum decomposition

$$
R=R_{(1)}+R_{(2)}+\cdots+R_{(\bar{m})},
$$

where $R_{(l)}=R_{(l), 1} \oplus \cdots \oplus R_{(l), s}$. Here it is understood that $R_{(l), i}=0$ if $m_{i}<l \leq \bar{m}$. We conclude that

$$
\begin{aligned}
& X_{\text {red }}(z)=K(z) z^{D} z^{L}, \quad K(z) \sim I+\sum_{j=1}^{\infty} K_{j} z^{-j}, \quad z \rightarrow \infty \text { in } \bar{S}(\alpha, \beta), \\
& K(z):=K_{1}(z) \oplus \cdots \oplus K_{s}(z), \quad K_{j}=K_{1}^{(j)} \oplus \cdots \oplus K_{s}^{(j)} .
\end{aligned}
$$

Hence, there is a fundamental solution of (2.1) at $t=0$, of the form

$$
\stackrel{\circ}{Y}(z):=G(z, 0) \Delta_{0} K(z) z^{D} z^{L} e^{\Lambda z} .
$$

This is rewritten as,

$$
\stackrel{\circ}{Y}(z)=\Delta_{0} \mathcal{G}(z) z^{D} z^{L} e^{\Lambda z}
$$

14 The gauge transformation $\tilde{Y}(z, 0)=\Delta_{0} X(z)$, of the system $(2.13)$ at $t=0$ yields,

$$
\frac{d X}{d z}=\mathcal{B}(z) \tilde{X}, \quad \mathcal{B}(z):=\Delta_{0}^{-1} B(z, 0) \Delta_{0}, \quad \mathcal{B}(z) \sim \Lambda+\frac{J}{z}+\sum_{k=2}^{\infty} \frac{\mathcal{B}_{k}}{z^{k}}, \quad \mathcal{B}_{k}:=\Delta_{0}^{-1} B_{k}(0) \Delta_{0} .
$$


where $\mathcal{G}(z):=\Delta_{0}^{-1} G(z, 0) \Delta_{0} K(z)$. Clearly,

$$
\mathcal{G}(z) \sim I+\sum_{k=1}^{\infty} \stackrel{\circ}{F}_{k} z^{-k}:=\left(I+\sum_{k=1}^{\infty} \Delta_{0}^{-1} G_{k}(0) \Delta_{0}\right)\left(I+\sum_{k=1}^{\infty} K_{k} z^{-k}\right), \quad z \rightarrow \infty \text { in } \bar{S}(\alpha, \beta) .
$$

The results above can be summarized in the following theorem:

Theorem 4.1. Consider the system (2.1) satisfying the assumptions of Theorem 2.1. There exist an invertible block-diagonal matrix $\Delta_{0}$ and a matrix $\mathcal{G}(z)$, holomorphic for $|z|>N, z \in \bar{S}(\alpha, \beta)$, with asymptotic expansion

$$
\mathcal{G}(z) \sim I+\sum_{k=1}^{\infty} \stackrel{\circ}{F}_{k} z^{-k}, \quad z \rightarrow \infty, \quad z \in \bar{S}(\alpha, \beta),
$$

such that the gauge transformation $Y(z, 0)=\Delta_{0} \mathcal{G}(z) \mathcal{Y}(z)$ transforms (2.1) at $t=0$ into a blockeddiagonal system

$$
\frac{d \mathcal{Y}}{d z}=\left[\Lambda+\frac{1}{z}\left(J+\frac{R_{(1)}}{z}+\cdots+\frac{R_{(\bar{m})}}{z^{\bar{m}}}\right)\right] \mathcal{Y}, \quad J=J_{1} \oplus \cdots \oplus J_{s}
$$

where $J_{i}$ is a Jordan form of $A_{i i}^{(1)}(0)=\widehat{A}_{i i}^{(1)}(0), 1 \leq i \leq s$, and the $R_{(l)}, 1 \leq l \leq \bar{m}$ are defined in (4.7)-(4.9). The system (4.12) has a fundamental solution $\mathcal{Y}(z)=z^{D} z^{L} e^{\Lambda z}$, hence (2.1) restricted at $t=0$ has a fundamental solution,

$$
\stackrel{\circ}{Y}(z)=\Delta_{0} \mathcal{G}(z) z^{D} z^{L} e^{\Lambda z} .
$$

The matrices $D, L$ are defined in (4.5), (4.6) and (4.8). The matrix $\Delta_{0}$ satisfies

$$
\Delta_{0}^{-1} A_{1}(0) \Delta_{0}=\left[\begin{array}{cccc}
J_{1} & * & * & * \\
* & J_{2} & & * \\
* & * & \ddots & * \\
* & * & * & J_{s}
\end{array}\right] \text {. }
$$

Remark 4.1. Observe that (4.13) does not solve (2.1) for $t \neq 0$.

Definition 4.1. The matrix

$$
\stackrel{\circ}{Y}_{F}(z):=\Delta_{0} F(z) z^{D} z^{L} e^{\Lambda z}, \quad F(z):=I+\sum_{k=1}^{\infty} \stackrel{\circ}{F}_{k} z^{-k},
$$

is called $a$ formal solution of (2.1) for $t=0$ and $A_{0}(0)=\Lambda$.

Notice that we use the notation $\stackrel{\circ}{Y}$ for solutions of the system with $t=0$. For fixed $\Delta_{0}, D, L$ and $\Lambda$ the formal solution is in general not unique. See Corollary 4.1.

We note that (4.14) can be transformed into a formal solution with the structure described in [3], but the specific form (4.14) is more refined and is obtainable by an explicit construction from the differential system (see also Section 4.1 below).

4.1. Explicit computation of the $\stackrel{\circ}{F}_{k}$ 's and $R$ of (4.11) and (4.12). Uniqueness of Formal Solutions. We present the computation of the $F_{k}$ 's in (4.11) and $R$ in (4.9). This serves for two reasons. First, the details of the computation in itself will be used later, starting from section 4.2. Second, it yields the Corollary 4.1 below concerning the (non-)uniqueness of formal solutions. Consider the gauge transformation $Y=\Delta_{0} \widehat{X}$ at $t=0$, which transforms (2.1) into

$$
\begin{aligned}
& \frac{d \widehat{X}}{d z}=\left(\Delta_{0}^{-1} A(z, 0) \Delta_{0}\right) \widehat{X}(z), \\
& \Delta_{0}^{-1} A(z, 0) \Delta_{0}=\Lambda+\sum_{j=1}^{\infty} \mathcal{A}_{j} z^{-j}, \quad \mathcal{A}_{j}:=\Delta_{0}^{-1} A_{j}(0) \Delta_{0} .
\end{aligned}
$$


The recurrence equations (2.17), (2.18) become (using $F_{l}$ instead of $G_{l}$ ),

$$
\begin{gathered}
\Lambda F_{1}-F_{1} \Lambda=-\mathcal{A}_{1}+B_{1}, \quad \text { with } \operatorname{diag}\left(\mathcal{A}_{1}\right)=J \\
\Lambda F_{l}-F_{l} \Lambda=\left[\sum_{j=1}^{l-1}\left(F_{j} B_{l-j}-\mathcal{A}_{l-j} F_{j}\right)-\mathcal{A}_{l}\right]-(l-1) F_{l-1}+B_{l} .
\end{gathered}
$$

Proposition 4.1. (4.15)-(4.16) admit a solution $\left\{F_{k}\right\}_{k \geq 1},\left\{B_{k}\right\}_{k \geq 1}$ which satisfies,

$$
\begin{aligned}
& B_{1}=J, \\
& B_{2}=R_{(1)}, \quad \ldots, \quad B_{\bar{m}+1}=R_{(\bar{m})}, \\
& B_{k}=0 \text { for any } k \geq \bar{m}+2,
\end{aligned}
$$

where $R_{(l)}=R_{(l), 1} \oplus \cdots \oplus R_{(l), s}$, and each $R_{(l), i}$ is as in (4.7). The $F_{k}$ 's so obtained are exactly the coefficients $\stackrel{\circ}{F}_{k}$ of the asymptotic expansion of the gauge transformation (4.11), which yields (4.12).

Proof: Let $\mathcal{K}_{l}:=\left[\sum_{j=1}^{l-1}\left(F_{j} B_{l-j}-\mathcal{A}_{l-j} F_{j}\right)-\mathcal{A}_{l}\right]$, and rewrite (4.15) and (4.16) in blocks $i, j$ :

- $\underline{\text { For } l=1}([i, j]$ is the block index, $1 \leq i, j \leq s)$ :

- For $l \geq 2$ :

$$
\Lambda F_{1}-F_{1} \Lambda=-\mathcal{A}_{1}+B_{1} \quad \Longrightarrow \quad\left(\lambda_{i}-\lambda_{j}\right) F_{i j}^{(1)}=-\mathcal{A}_{i j}^{(1)}+B_{i j}^{(1)}
$$

$$
\Lambda F_{l}-F_{l} \Lambda=\mathcal{K}_{j}-(l-1) F_{l-1}+B_{l} \quad \Longrightarrow \quad\left(\lambda_{i}-\lambda_{j}\right) F_{i j}^{(l)}=\mathcal{K}_{i j}^{(l)}-(l-1) F_{i j}^{(l-1)}+B_{i j}^{(l)} .
$$

- For $l=1$ we find:

- If $i=j$ :

- If $i \neq j$ :

$$
B_{i i}^{(1)}=\mathcal{A}_{i i}^{(1)} \equiv J_{i}, \quad F_{i i}^{(1)} \text { not determined. }
$$

$$
F_{i j}^{(1)}=-\frac{\mathcal{A}_{i j}^{(1)}}{\lambda_{i}-\lambda_{j}}, \quad B_{i j}^{(1)}=0
$$

- For $l \geq 2$ we find:

- If $i \neq j$ :

$$
F_{i j}^{(l)}=\left(\lambda_{i}-\lambda_{j}\right)^{-1}\left(\mathcal{K}_{i j}^{(l)}-(l-1) F_{i j}^{(l-1)}\right), \quad B_{i j}^{(l)}=0 .
$$

In the r.h.s. matrix entries of $F_{1}, \ldots, F_{l-1}$ appear, therefore the equation determines $F_{i j}^{(l)}$.

- If $i=j$ :

$$
0=\mathcal{K}_{i i}^{(l)}-(l-1) F_{i i}^{(l-1)}+B_{i i}^{(l)}
$$

We observe that in $\mathcal{K}_{i i}^{(l)}$ the matrix entries of $F_{1}, \ldots, F_{l-1}$ appear, including the entry $F_{i i}^{(l-1)}$. Keeping into account that $B_{1}=\mathcal{A}_{11}^{(1)} \oplus \cdots \oplus \mathcal{A}_{s s}^{(1)}$, we explicitly write (4.17):

$$
\begin{gathered}
(l-1) F_{i i}^{(l-1)}=\sum_{k=1}^{s}\left(F_{i k}^{(l-1)} B_{k i}^{(1)}-\mathcal{A}_{i k}^{(1)} F_{k i}^{(l-1)}\right)+\sum_{j=1}^{l-2}\left(F_{j} B_{l-j}-\mathcal{A}_{l-j} F_{j}\right)_{[i, i]}-\mathcal{A}_{i i}^{(l)}+B_{i i}^{(l)}= \\
=F_{i i}^{(l-1)} \mathcal{A}_{i i}^{(1)}-\mathcal{A}_{i i}^{(1)} F_{i i}^{(l-1)}-\sum_{k \neq i} \mathcal{A}_{i k}^{(1)} F_{k i}^{(l-1)}+\sum_{j=1}^{l-2}\left(F_{j} B_{l-j}-\mathcal{A}_{l-j} F_{j}\right)_{[i, i]}-\mathcal{A}_{i i}^{(l)}+B_{i i}^{(l)} .
\end{gathered}
$$

Thus, keeping into account that $\mathcal{A}_{i i}^{(1)}=J_{i}$, the above is rewritten as follows:

$$
\left(J_{i}+l-1\right) F_{i i}^{(l-1)}-F_{i i}^{(l-1)} J_{i}=-\sum_{k \neq i} \mathcal{A}_{i k}^{(1)} F_{k i}^{(l-1)}+\sum_{j=1}^{l-2}\left(F_{j} B_{l-j}-\mathcal{A}_{l-j} F_{j}\right)_{[i, i]}-\mathcal{A}_{i i}^{(l)}+B_{i i}^{(l)} .
$$


In the r.h.s. every term is determined by previous steps (diagonal elements $F_{j j}^{(k)}$ appear up to $k \leq l-2$ ), except for $B_{i i}^{(l)}$, which is still undetermined. (4.18) splits into the blocks inherited from $J_{i}=J_{1}^{(i)} \oplus \cdots \oplus$ $J_{h_{i}}^{(i)}$. Let the eigenvalues of $J_{i}$ be $\mu_{1}^{(i)}, \ldots, \mu_{h_{i}}^{(i)}, h_{i} \leq p_{i}$. Then (for $l \geq 2$ ),

$$
\begin{aligned}
& \left(\mu_{a}^{(i)}+l-1+H_{r_{a}}\right)\left[F_{i i}^{(l-1)}\right]_{a b}-\left[F_{i i}^{(l-1)}\right]_{a b}\left(\mu_{b}^{(i)}+H_{r_{b}}\right)= \\
= & {\left[-\sum_{k \neq i} \mathcal{A}_{i k}^{(1)} F_{k i}^{(l-1)}+\sum_{j=1}^{l-2}\left(F_{j} B_{l-j}-\mathcal{A}_{l-j} F_{j}\right)_{[i, i]}-\mathcal{A}_{i i}^{(l)}+B_{i i}^{(l)}\right]_{a b} . }
\end{aligned}
$$

Here $[\cdots]_{a b}$ denotes a block, with $1 \leq a, b \leq h_{i}$.

- If $\mu_{b}^{(i)}-\mu_{a}^{(i)}=l-1$, the l.h.s. of (4.19) is $H_{r_{a}}\left[F_{i i}^{(l-1)}\right]_{a b}-\left[F_{i i}^{(l-1)}\right]_{a b} H_{r_{b}}$. The homogeneous equation $H_{r_{a}}\left[F_{i i}^{\overline{(l-1)}]_{a b}-\left[F_{i i}^{(l-1)}\right]_{a b}} H_{r_{b}}=0\right.$ has non trivial solutions, depending on parameters, since the matrices $H_{r_{a}}$ and $H_{r_{b}}$ have common eigenvalue. One can then choose $F_{i i}$ to be a solution of the homogeneous equation, and determine $\left[B_{i i}^{(l)}\right]_{a b} \neq 0$ by imposing that the r.h.s. of (4.19) is equal to 0.

- If $\underline{\mu_{b}^{(i)}-\mu_{a}^{(i)} \neq l-1}$, the choice $\left[B_{i i}^{(l)}\right]_{a b}=0$ is possible and $\left[F_{i i}^{(l-1)}\right]_{a b}$ is determined.

We conclude that

$$
\left[B_{i i}^{(l+1)}\right]_{a b} \neq 0 \quad \text { only if } \quad \mu_{b}^{(i)}-\mu_{a}^{(i)}=l>0 \text { integer. }
$$

This means that $\left[B_{i i}^{(l+1)}\right]_{a b}=\left[R_{(l), i}\right]_{a b}$.

Corollary 4.1 (Uniqueness of Formal Solution at $t=0$ ). A formal solution (4.14) with given $\Delta_{0}$, D, $L, \Lambda$ is unique if and only if for any $1 \leq i \leq s$ the eigenvalues of $\widehat{A}_{i i}^{(1)}(0)$ do not differ by a non-zero integer.

Proof: Computations above show that $\left\{F_{k}\right\}_{k=1}^{\infty}$ is not uniquely determined if and only if some $\mu_{b}^{(i)}-\mu_{a}^{(i)}=$ $l-1$, for some $l \geq 2$, some $i \in\{1,2, \ldots, s\}$, and some $a, b$.

4.2. Special sub-case with $R=0, J$ diagonal, $\Delta_{0}=I$. A sub-case is very important for the discussion to come, occurring when $\Delta_{0}=I$ and $A_{i i}^{(1)}(0)$ is diagonal. Clearly, if $\Delta_{0}=I$, then $J_{i}=A_{i i}^{(1)}(0)$. Hence, if $\Delta_{0}=I$, then $J$ is diagonal if and only if $\left(\widehat{A}_{i i}^{(1)}(0)\right)_{p q}=0$ for any $1 \leq p \neq q \leq p_{i}$.

Proposition 4.2. There exists a fundamental solution (4.13) at $t=0$ in a simpler form

$$
\stackrel{\circ}{Y}(z)=\mathcal{G}(z) z^{B_{1}(0)} e^{\Lambda z},
$$

with $\Delta_{0}=I, J=B_{1}(0)=\operatorname{diag}\left(A_{1}(0)\right)$ diagonal, and

$$
\mathcal{G}(z) \sim I+\sum_{k=1}^{\infty} \stackrel{\circ}{F}_{k} z^{-k}, \quad z \rightarrow \infty \quad \text { in } \bar{S}(\alpha, \beta),
$$

if and only if the following conditions hold:

- For every $i \in\{1,2, \ldots, s\}$, and every $p, q$, with $1 \leq p \neq q \leq p_{i}$, then

$$
\left(\widehat{A}_{i i}^{(1)}(0)\right)_{p q}=0 \text {. }
$$

- If $\left(\widehat{A}_{i i}^{(1)}(0)\right)_{p p}-\left(\widehat{A}_{i i}^{(1)}(0)\right)_{q q}+l-1=0$, for some $l \geq 2$, some $i \in\{1,2, \ldots, s\}$, and some diagonal entries $\left(\widehat{A}_{i i}^{(1)}(0)\right)_{p p},\left(\widehat{A}_{i i}^{(1)}(0)\right)_{q q}$, then

$$
\sum_{k \neq i}^{s}\left(\widehat{A}_{i k}^{(1)}(0) \stackrel{\circ}{F}_{k i}^{(l-1)}\right)_{p q}+\sum_{j=1}^{l-2} \sum_{k=1}^{s}\left(\widehat{A}_{i k}^{(l-j)}(0) \stackrel{\circ}{F}_{k i}^{(j)}\right)_{p q}+\left(\widehat{A}_{i i}^{(l)}(0)\right)_{p q}=0
$$


for those values of $l, i, p$ and $q$.

Proof: We only need to clarify (4.23), while (4.22) has already been motivated. We solve (4.15), (4.16) when $\Delta_{0}=I$, namely (recall that $\widehat{A}_{j}(0) \equiv A_{j}(0)$ ) (we write $F_{l}$, as in (4.15), (4.16), but it is clear that the result of the computation will be the $\stackrel{\circ}{F}_{l}$ appearing in (4.21)):

$$
\begin{aligned}
& \Lambda F_{1}-F_{1} \Lambda=-\widehat{A}_{1}(0)+B_{1}, \\
& \Lambda F_{l}-F_{l} \Lambda=\left[\sum_{j=1}^{l-1}\left(F_{j} B_{l-j}-\widehat{A}_{l-j}(0) F_{j}\right)-\widehat{A}_{l}(0)\right]-(l-1) F_{l-1}+B_{l} .
\end{aligned}
$$

At level $l=1$ :

At level $l \geq 2$,

$$
B_{1}=\operatorname{diag} \widehat{A}_{1}(0), \quad F_{i j}^{(1)}=-\frac{\widehat{A}_{i j}(0)}{\lambda_{i}-\lambda_{j}}
$$

$$
F_{i j}^{(l)}=\frac{\mathcal{K}_{i j}^{(l)}-(l-1) F_{i j}^{(l-1)}}{\lambda_{i}-\lambda_{j}}, \quad B_{i j}^{(l)}=0
$$

where $\mathcal{K}_{l}=\left[\sum_{j=1}^{l-1}\left(F_{j} B_{l-j}-\widehat{A}_{l-j}(0) F_{j}\right)-\widehat{A}_{l}(0)\right]$. Formula (4.19) reads

$$
\left(\mu_{a}^{(i)}-\mu_{b}^{(i)}+l-1\right)\left[F_{i i}^{(l-1)}\right]_{a b}=\left[-\sum_{k \neq i} \widehat{A}_{i k}^{(1)}(0) F_{k i}^{(l-1)}+\sum_{j=1}^{l-2}\left(F_{j} B_{l-j}-\widehat{A}_{l-j}(0) F_{j}\right)_{[i, i]}-\widehat{A}_{i i}^{(l)}(0)+B_{i i}^{(l)}\right]_{a b} .
$$

Indices above are block indices. The above can be re-written in terms of the matrix entries,

$$
\begin{aligned}
& \left(\left(\widehat{A}_{i i}^{(1)}(0)\right)_{p p}-\left(\widehat{A}_{i i}^{(1)}(0)\right)_{q q}+l-1\right)\left(F_{i i}^{(l-1)}\right)_{p q}= \\
& \quad=\left[-\sum_{k \neq i} \widehat{A}_{i k}^{(1)}(0) F_{k i}^{(l-1)}+\sum_{j=1}^{l-2}\left(F_{j} B_{l-j}-\widehat{A}_{l-j}(0) F_{j}\right)_{[i, i]}-\widehat{A}_{i i}^{(l)}(0)+B_{i i}^{(l)}\right]_{\text {entry } p q} .
\end{aligned}
$$

- If $\left(\widehat{A}_{i i}^{(1)}(0)\right)_{p p}-\left(\widehat{A}_{i i}^{(1)}(0)\right)_{q q}+l-1 \neq 0$, choose $B_{i i}^{(l)}=0$ and determine $\left(F_{i i}^{(l-1)}\right)_{p q}$.

- If $\left(\widehat{A}_{i i}^{(1)}(0)\right)_{p p}-\left(\widehat{A}_{i i}^{(1)}(0)\right)_{q q}+l-1=0$, by induction assume that the $B_{l-j}=0$. Then the equation is satisfied for any $\left(F_{i i}^{(l-1)}\right)_{p q}$ and for

$$
\left(B_{i i}^{(l)}\right)_{p q}=\left[\sum_{k \neq i} \widehat{A}_{i k}^{(1)}(0) F_{k i}^{(l-1)}+\sum_{j=1}^{l-2}\left(\widehat{A}_{l-j}(0) F_{j}\right)_{\text {block }[i, i]}+\widehat{A}_{i i}^{(l)}(0)\right]_{\text {entry } p q} .
$$

Then, if we impose that $\left(B_{i i}^{(l)}\right)_{p q}=0$ we obtain the necessary and sufficient condition (4.23). The proof by induction is justified because at the first step, namely $l=2$, we need to solve

$$
\left(\left(\widehat{A}_{i i}^{(1)}(0)\right)_{p p}-\left(\widehat{A}_{i i}^{(1)}(0)\right)_{q q}+1\right)\left(F_{i i}^{(1)}\right)_{p q}=-\sum_{k \neq i}^{n}\left(\widehat{A}_{i k}^{(1)}(0) F_{k i}^{(1)}\right)_{p q}-\left(\widehat{A}_{i i}^{(2)}(0)\right)_{p q}+\left(B_{i i}^{(2)}\right)_{p q} .
$$

If $\left(\widehat{A}_{i i}^{(1)}(0)\right)_{p p}-\left(\widehat{A}_{i i}^{(1)}(0)\right)_{q q}+1 \neq 0$, the above has a unique solution for any choice of $\left(B_{i i}^{(2)}\right)_{p q}$. We choose $\left(B_{i i}^{(2)}\right)_{p q}=0$. If $\left.\widehat{A}_{i i}^{(1)}(0)\right)_{p p}-\left(\widehat{A}_{i i}^{(1)}(0)\right)_{q q}+1=0$, the equation leaves the choice of $\left(F_{i i}^{(1)}\right)_{p q}$ free, and determines

$$
\left(B_{i i}^{(2)}\right)_{p q}=\sum_{k \neq i}^{n}\left(\widehat{A}_{i k}^{(1)}(0) F_{k i}^{(1)}\right)_{p q}+\left(\widehat{A}_{i i}^{(2)}(0)\right)_{p q}=-\sum_{k \neq i}^{n} \frac{\left(\widehat{A}_{i k}^{(1)}(0) \widehat{A}_{k i}^{(1)}(0)\right)_{p q}}{\lambda_{k}-\lambda_{i}}+\left(\widehat{A}_{i i}^{(2)}(0)\right)_{p q} .
$$

We can choose $\left(B_{i i}^{(2)}\right)_{p q}=0$ if and only if

$$
\left(\widehat{A}_{i i}^{(2)}(0)\right)_{p q}=\sum_{k \neq i}^{n} \frac{\left(\widehat{A}_{i k}^{(1)}(0) \widehat{A}_{k i}^{(1)}(0)\right)_{p q}}{\lambda_{k}-\lambda_{i}}
$$


which is precisely (4.23) for $l=2$.

\section{Solutions for $t \in \mathcal{U}_{\epsilon_{0}}(0)$ with $A_{0}(t)$ Holomorphically Diagonalisable.}

In the previous section, we have constructed fundamental solutions at the coalescence point $t=0$. Now, we let $t$ vary in $\mathcal{U}_{\epsilon_{0}}(0)$. In Sibuya Theorem, $\widehat{A}_{0}(t)=\widehat{A}_{11}^{(0)}(t) \oplus \cdots \oplus \widehat{A}_{s s}^{(0)}(t)$ is neither diagonal nor in Jordan form, except for $t=0$. $A_{0}(t)$ admits a Jordan form at each point of $\mathcal{U}_{\epsilon_{0}}(t)$, but in general this similarity is not realizable by a holomorphic transformation. In order to procede, we need the following fundamental assumption, already stated in the Introduction.

Assumption 1: For $|t| \leq \epsilon_{0}$ sufficiently small and such that Lemma 2.1 and Theorem 2.1 apply, we assume that $A_{0}(t)$ is holomorphically similar to a diagonal form $\Lambda(t)$, namely there exists a holomorphic invertible $G_{0}(t)$ for $|t| \leq \epsilon_{0}$ such that

$$
G_{0}(t)^{-1} A_{0}(t) G_{0}(t)=\Lambda(t) \equiv \operatorname{diag}\left(u_{1}(t), u_{2}(t), \ldots, u_{n}(t)\right),
$$

with $A_{0}(0)=\Lambda, G_{0}(0)=I$.

Remark 5.1. Assumption 1 is equivalent to the assumption that $A_{0}(t)$ is holomorphically similar to its Jordan form. The requirement implies by continuity that the Jordan form is diagonal, being equal to $\Lambda=\Lambda(0)$ at $t=0$.

With Assumption 1, we can represent the eigenvalues as well defined holomorphic functions $u_{1}(t)$, $u_{2}(t), \ldots, u_{n}(t)$ such that

$$
\begin{aligned}
u_{1}(0)=\cdots=u_{p_{1}}(0) & =\lambda_{1}, \\
u_{p_{1}+1}(0)=\cdots=u_{p_{1}+p_{2}}(0) & =\lambda_{2}, \\
& \\
u_{p_{1}+\cdots+p_{s-1}+1}(0)=\cdots=u_{p_{1}+\cdots+p_{s-1}+p_{s}}(0) & =\lambda_{s} .
\end{aligned}
$$

Moreover,

$$
\Lambda(t)=\Lambda_{1}(t) \oplus \Lambda_{2}(t) \oplus \cdots \oplus \Lambda_{s}(t)
$$

where $\Lambda_{1}(t), \ldots, \Lambda_{s}(t)$ are diagonal matrices of dimensions respectively $p_{1}, \ldots, p_{s}$, such that $\Lambda_{j}(t) \rightarrow \lambda_{j} I_{p_{j}}$ for $t \rightarrow 0, j=1, \ldots, s$. For example, $\Lambda_{1}(t)=\operatorname{diag}\left(u_{1}(t), \ldots, u_{p_{1}}(t)\right)$, and so on. Any two matrices $\Lambda_{i}(t)$ and $\Lambda_{j}(t)$ have no common eigenvalues for $i \neq j$ and small $\epsilon_{0}$.

The coalescence locus in $\mathcal{U}_{\epsilon_{0}}(0)$ is explicitly written as follows

$$
\begin{aligned}
& \Delta:=\bigcup_{a \neq b}\left\{t \in \mathbb{C}^{m} \text { such that: }|t| \leq \epsilon_{0} \text { and } u_{a}(t)=u_{b}(t)\right\} . \\
& a, b=1, \ldots, m
\end{aligned}
$$

We can also write

$$
\Delta=\bigcup_{i=1}^{s} \Delta_{i}
$$

where $\Delta_{i}$ is the coalescence locus of $\Lambda_{i}(t)$. For $m=1, \Delta$ is a finite set of isolated points.

Improvement of Theorem 2.1: With the same assumptions and notations as of Theorem 2.1, if Assumption 1 holds, then

$$
B(z, t) \sim \Lambda(t)+\sum_{k \geq 1} B_{k}(t) z^{-k}, \quad z \rightarrow \infty \text { in } \bar{S}(\alpha, \beta) .
$$

With Assumption 1, we can replace the gauge trasfromation (3.1) with

$$
\tilde{Y}(z, t)=e^{\Lambda(t) z} Y_{\text {red }}(z, t) .
$$

Since $\widehat{A}_{0}(t)=\Lambda(t)$, then $B_{\text {red }}(z, t) \sim \sum_{k=1}^{\infty} B_{k}(t) z^{-k+1}$. Hence the reduced system (3.2) is Fuchsian also for $t \neq 0$. The recursive relations (2.17) and (2.18) become $B_{0}(t)=\Lambda(t)$ for $l=0$, and: 
$\underline{\text { For } l=1}$ :

$\underline{\text { For } l \geq 2:}$

$$
\Lambda(t) G_{1}-G_{1} \Lambda(t)=-\widehat{A}_{1}(t)+B_{1}
$$

$$
\Lambda(t) G_{l}-G_{l} \Lambda(t)=\left[\sum_{j=1}^{l-1}\left(G_{j} B_{l-j}-\widehat{A}_{l-j}(t) G_{j}\right)-\widehat{A}_{l}(t)\right]-(l-r) G_{l-r}+B_{l} .
$$

As for Theorem 2.1, the choice which yields holomorphic $G_{l}(t)$ 's and $B_{l}(t)$ 's is (2.19) and (2.20). Generally speaking, it is not possible to choose the $B_{l}(t)$ 's diagonal for $l \geq 2$, because such a choice would give $G_{k}(t)$ 's diverging at the locus $\Delta$.

5.1. Fundamental Solution in a neighbourhood of $t_{0} \notin \Delta$, with Assumption 1. Let Assumption 1 hold. Theorem 2.1 has been formulated in a neighbourhood of $t=0$, with block partition of $A_{0}(0)=$ $\Lambda_{1} \oplus \cdots \oplus \Lambda_{s}$. Theorem 2.1 can also be formulated in a neighbourhood (polydisc) of a point $t_{0} \in \mathcal{U}_{\epsilon_{0}}(0) \backslash \Delta$, of the form

$$
\begin{aligned}
& \mathcal{U}_{\rho_{0}}\left(t_{0}\right):=\left\{t \in \mathbb{C}|| t-t_{0} \mid \leq \rho_{0}\right\} \subset \mathcal{U}_{\epsilon_{0}}(0), \\
& \mathcal{U}_{\rho_{0}}\left(t_{0}\right) \cap \Delta=\emptyset,
\end{aligned}
$$

where $\Lambda(t)$ has distinct eigenvalues, provided that $\rho_{0}>0$ is small enough. In order to do this, we need to introduce sectors. To this end, consider a fixed point $t_{*}$ in $\mathcal{U}_{\epsilon_{0}}(0)$, and the eigenvalues $u_{1}\left(t_{*}\right), \ldots$, $u_{n}\left(t_{*}\right)$ of $\Lambda\left(t_{*}\right)$. We introduce an admissible direction $\eta^{\left(t_{*}\right)}$ such that

$$
\eta^{\left(t_{*}\right)} \neq \arg _{p}\left(u_{a}\left(t_{*}\right)-u_{b}\left(t_{*}\right)\right) \bmod (2 \pi), \quad \forall 1 \leq a \neq b \leq n .
$$

There are $2 \mu_{t_{*}}$ determinations satisfying $\eta^{\left(t_{*}\right)}-2 \pi<\widehat{\arg }\left(u_{a}\left(t_{*}\right)-u_{b}\left(t_{*}\right)\right)<\eta^{\left(t_{*}\right)}$. They will be numbered as

$$
\eta^{\left(t_{*}\right)}>\eta_{0}^{\left(t_{*}\right)}>\cdots>\eta_{2 \mu^{\left(t_{*}\right)}-1}>\eta^{\left(t_{*}\right)}-2 \pi .
$$

Correspondingly, we introduce the directions

$$
\tau^{\left(t_{*}\right)}:=\frac{3 \pi}{2}-\eta^{\left(t_{*}\right)}, \quad \tau_{\nu}^{\left(t_{*}\right)}=\frac{3 \pi}{2}-\eta_{\nu}^{\left(t_{*}\right)}, \quad 0 \leq \nu \leq 2 \mu_{t_{*}}-1,
$$

satisfying

$$
\tau^{\left(t_{*}\right)}<\tau_{0}^{\left(t_{*}\right)}<\tau_{1}^{\left(t_{*}\right)}<\cdots<\tau_{2 \mu_{t_{*}}-1}^{\left(t_{*}\right)}<\tau^{\left(t_{*}\right)}+2 \pi
$$

The following relation defines $\tau_{\sigma}^{\left(t_{*}\right)}$ for any $\sigma \in \mathbb{Z}$, represented as $\sigma=\nu+k \mu_{t_{*}}$ :

$$
\tau_{\nu+k \mu_{t_{*}}}:=\tau_{\nu}^{\left(t_{*}\right)}+k \pi, \quad \nu \in\left\{0,1, \ldots, \mu_{t_{*}}-1\right\}, \quad k \in \mathbb{Z} .
$$

Finally, we introduce the sectors

$$
\mathcal{S}_{\sigma}^{\left(t_{*}\right)}:=S\left(\tau_{\sigma}^{\left(t_{*}\right)}-\pi, \tau_{\sigma+1}^{\left(t_{*}\right)}\right), \quad \sigma \in \mathbb{Z} .
$$

Theorem 2.1 in a neighbourhood of $t_{0}$ becomes:

Theorem 5.1. Let Assumption 1 hold and let $t_{0} \in \mathcal{U}_{\epsilon_{0}}(0) \backslash \Delta$. Pick up a sector $\mathcal{S}_{\sigma}^{\left(t_{0}\right)}=S\left(\tau_{\sigma}^{\left(t_{0}\right)}-\pi, \tau_{\sigma+1}^{\left(t_{0}\right)}\right)$, $\sigma \in \mathbb{Z}$, as above. For any closed sub-sector

$$
\bar{S}^{\left(t_{0}\right)}(\alpha, \beta):=\left\{z \in \mathcal{R} \mid \tau_{\sigma}^{\left(t_{0}\right)}-\pi<\alpha \leq \arg z \leq \beta<\tau_{\sigma+1}^{\left(t_{0}\right)}\right\} \subset \mathcal{S}_{\sigma}^{\left(t_{0}\right)},
$$

there exist a sufficiently large positive number $N$, a sufficiently small positive number $\rho$ and an invertible matrix valued function $G(z, t)$ with the following properties:

i) $G(z, t)$ is holomorphic in $(z, t)$ for $|z| \geq N, z \in \bar{S}^{\left(t_{0}\right)}(\alpha, \beta),\left|t-t_{0}\right| \leq \rho$;

ii) $G(z, t)$ has uniform asymptotic expansion for $\left|t-t_{0}\right| \leq \rho$, with holomorphic coefficients $G_{k}(t)$ :

$$
G(z, t) \sim I+\sum_{k=1}^{\infty} G_{k}(t) z^{-k}, \quad z \rightarrow \infty, \quad z \in \bar{S}^{\left(t_{0}\right)}(\alpha, \beta),
$$


iii) The gauge transformation

$$
Y(z, t)=G_{0}(t) G(z, t) \tilde{Y}(z, t),
$$

reduces the initial system (2.1) to

$$
\frac{d \widetilde{Y}}{d z}=B(z, t) \tilde{Y}
$$

where $B(z, t)$ is a diagonal holomorphic matrix function of $(z, t)$ in the domain

$|z| \geq N, z \in \bar{S}(\alpha, \beta),\left|t-t_{0}\right| \leq \rho$, with uniform asymptotic expansion and holomorphic coefficients:

$$
B(z, t) \sim \Lambda(t)+\sum_{k=1}^{\infty} B_{k}(t) z^{-k}, \quad z \rightarrow \infty, \quad z \in \bar{S}^{\left(t_{0}\right)}(\alpha, \beta) .
$$

In particular, $B_{1}(t)=\operatorname{diag} \widehat{A}_{1}(t)$.

Remark 5.2. $\bar{S}^{\left(t_{0}\right)}(\alpha, \beta)$ is not the same $\bar{S}(\alpha, \beta)$ of Theorem 2.1 (the latter should be denoted $\bar{S}^{(0)}(\alpha, \beta)$ for consistency of notations). The matrices $G(z, t)$ and $B(z, t)$ are not the same of Theorem 2.1. On the other hand, $G_{0}(t)$ is the same, by Assumption 1.

As before, we let $B_{\text {red }}(z, t)=z(B(z, t)-\Lambda(t))$. Then the system (2.1) has a fundamental matrix solution

$$
Y(z, t)=G_{0}(t) \mathcal{G}(z, t) z^{B_{1}(t)} e^{\Lambda(t) z},
$$

where $\mathcal{G}(z, t)=G(z, t) K(z, t)$, and

$$
K(z, t)=\exp \left\{\int_{\infty}^{z} \frac{B_{r e d}(\zeta, t)-B_{1}(t)}{\zeta} d \zeta\right\} \sim \exp \left\{\sum_{k=2}^{\infty} B_{k}(t) \frac{z^{-k+1}}{-k+1}\right\}=I+\sum_{j=1}^{\infty} K_{j}(t) z^{j},
$$

$z \rightarrow \infty$ in $\bar{S}(\alpha, \beta)$. This result is well known, see [37]. This proves the first part of the following

Corollary 5.1. The analogue of Theorem 5.1 holds with a new gauge transfromation $\mathcal{G}(z, t)$, enjoying the same asymptotic and analytic properties, such that $Y(z, t)=G_{0}(t) \mathcal{G}(z, t) \widetilde{Y}(z)$ transforms the system (2.1) into

$$
\frac{d \tilde{Y}}{d z}=\left(\Lambda(t)+\frac{B_{1}(t)}{z}\right) \tilde{Y}, \quad B_{1}(t)=\operatorname{diag} \widehat{A}_{1}(t)
$$

With the above choice, the system (2.1) has a fundamental solution,

$$
Y(z, t)=G_{0}(t) \mathcal{G}(z, t) z^{B_{1}(t)} e^{\Lambda(t) z}
$$

and $\mathcal{G}(z, t)$ is holomorphic for $z \in \bar{S}^{\left(t_{0}\right)}(\alpha, \beta),|z| \geq N$ and $\left|t-t_{0}\right| \leq \rho$, with expansion

$$
\mathcal{G}(z, t) \sim I+\sum_{k=1}^{\infty} F_{k}(t) z^{-k}
$$

for $z \rightarrow \infty$ in $\bar{S}^{\left(t_{0}\right)}(\alpha, \beta)$, uniformly in $\left|t-t_{0}\right| \leq \rho$. The coefficients $F_{k}(t)$ are uniquely determined and holomorphic on $\mathcal{U}_{\epsilon_{0}}(0) \backslash \Delta$.

Proof: The statement is clear from the previous construction. It is only to be justified that the $F_{k}(t)$ 's, $k \geq 1$, are holomorphic functions of $t \notin \Delta$ and uniquely determined. We solve (5.5) and (5.6) for the $F_{k}(t)$ 's, namely

$$
\begin{aligned}
& \Lambda(t) F_{1}-F_{1} \Lambda(t)=-\widehat{A}_{1}(t)+B_{1} \\
& \Lambda(t) F_{l}-F_{l} \Lambda(t)=\left[\sum_{j=1}^{l-1}\left(F_{j} B_{l-j}-\widehat{A}_{l-j}(t) F_{j}\right)-\widehat{A}_{l}(t)\right]-(l-1) F_{l-1}+B_{l} .
\end{aligned}
$$


It is convenient to use the notation $u_{1}(t), \ldots, u_{n}(t)$ for the distinct eigenvalues. Matrix entries are here denoted $a, b \in\{1,2, \ldots, n\}$. For $l=1$,

$$
\begin{array}{ll}
\left(F_{1}\right)_{a b}(t)=-\frac{\left(\widehat{A}_{1}\right)_{a b}(t)}{u_{a}(t)-u_{b}(t)}, \quad\left(B_{1}(t)\right)_{a b}=0, \quad a \neq b . \\
\left(B_{1}\right)_{a a}(t)=\left(\widehat{A}_{1}\right)_{a a}(t), \quad \Longrightarrow \quad B_{1}(t)=\operatorname{diag}\left(\widehat{A}_{1}(t)\right) .
\end{array}
$$

Now, impose that $B_{l}(t)=0$ for any $l \geq 2$. Hence, at level $l=2$ we get:

$$
\left(F_{1}\right)_{a a}(t)=-\sum_{b \neq a}\left(\widehat{A}_{1}\right)_{a b}(t)\left(F_{1}\right)_{b a}(t)-\left(\widehat{A}_{2}\right)_{a a}(t) .
$$

For any $l \geq 2$, we find:

$$
\begin{aligned}
\left(F_{l}\right)_{a b}(t) & =-\frac{1}{u_{a}(t)-u_{b}(t)}\left\{\left[\left(\widehat{A}_{1}\right)_{a a}(t)-\left(\widehat{A}_{1}\right)_{b b}(t)+l-1\right]\left(F_{l-1}\right)_{a b}(t)+\right. \\
& \left.+\sum_{\gamma \neq a}\left(\widehat{A}_{1}\right)_{a \gamma}(t)\left(F_{l-1}\right)_{\gamma b}(t)+\sum_{j=1}^{l-2}\left(\widehat{A}_{l-j}(t) F_{j}(t)\right)_{a b}+\left(\widehat{A}_{l}\right)_{a b}(t)\right\}, \quad a \neq b . \\
(l-1)\left(F_{l-1}\right)_{a a}(t) & =-\sum_{b \neq a}\left(\widehat{A}_{1}\right)_{a b}(t)\left(F_{l-1}\right)_{b a}(t)-\sum_{j=1}^{l-2}\left(\widehat{A}_{l-j}(t) F_{j}(t)\right)_{a a}-\left(\widehat{A}_{l}\right)_{a a}(t) .
\end{aligned}
$$

The above formulae show that the $F_{l}(t)$ are uniquely determined, and holomorphic away from $\Delta$.

The above result has two corollaries:

Proposition 5.1. The coefficients $F_{k}(t)$ in the expansion (5.10) are holomorphic at a point $t_{\Delta} \in \Delta$ if and only if there exists a neighbourhood of $t_{\Delta}$ where

$$
\left(\widehat{A}_{1}\right)_{a b}(t)
$$

and

$$
\left[\left(\widehat{A}_{1}\right)_{a a}(t)-\left(\widehat{A}_{1}\right)_{b b}(t)+l-1\right]\left(F_{l-1}\right)_{a b}(t)+\sum_{\gamma \neq a}\left(\widehat{A}_{1}\right)_{a \gamma}(t)\left(F_{l-1}\right)_{\gamma b}(t)+\sum_{j=1}^{l-2}\left(\widehat{A}_{l-j}(t) F_{j}(t)\right)_{a b}+\left(\widehat{A}_{l}\right)_{a b}(t)
$$

vanish as fast as $\mathcal{O}\left(u_{a}(t)-u_{b}(t)\right)$ in the neighbourhood, for those indexes $a, b \in\{1,2, \ldots, n\}$ such that $u_{a}(t)$ and $u_{b}(t)$ coalesce when $t$ approaches a point of $\Delta$ in the neighbourhood. In particular, the $F_{k}(t)$ 's are holomorphic in the whole $\mathcal{U}_{\epsilon_{0}}(0)$ if and only if (5.11) and (5.12) are zero along $\Delta$.

Remarkably, in the isomonodromic case, we will prove that if we just require vanishing of $\left(A_{1}\right)_{a b}(t)$ then all the complicated expressions (5.12) also vanish consequently.

Proposition 5.2. If the holomorphic conditions of Proposition 5.1 hold at $t=0$, then (4.22) and (4.23) are satisfied, with the choice

$$
\stackrel{\circ}{F}_{k}=F_{k}(0), \quad k \geq 1 .
$$

If moreover $\left(\widehat{A}_{1}(0)\right)_{a a}-\left(\widehat{A}_{1}(0)_{b b}+l-1 \neq 0\right.$ for every $l \geq 2$, then the above is the unique choice of the $\stackrel{\circ}{F}_{k}$ 's, according to Corollary 4.1 .

Expression (5.12) is a rational function of the matrix entries of $\widehat{A}_{1}(t), \ldots, \widehat{A}_{l}(t)$, since $F_{1}(t), \ldots, F_{l-1}(t)$ are expressed in terms of $\widehat{A}_{1}(t), \ldots, \widehat{A}_{l}(t)$. For example, for $l=2,(5.12)$ becomes

$$
\left(\left(\widehat{A}_{1}\right)_{b b}(t)-\left(\widehat{A}_{1}\right)_{a a}(t)-1\right) \frac{\left(\widehat{A}_{1}\right)_{a b}(t)}{u_{a}(t)-u_{b}(t)}+\left(\widehat{A}_{2}\right)_{a b}(t)-\sum_{\gamma \neq a} \frac{\left(\widehat{A}_{1}\right)_{a \gamma}(t)\left(\widehat{A}_{1}\right)_{\gamma b}(t)}{u_{\gamma}(t)-u_{b}(t)} .
$$


Example 5.1. The following system does not satisfy the vanishing conditions of Proposition 5.1

$$
\widehat{A}(z, t)=\left(\begin{array}{ll}
0 & 0 \\
0 & t
\end{array}\right)+\frac{1}{z}\left(\begin{array}{ll}
1 & 0 \\
t & 2
\end{array}\right), \quad \Delta=\{t \in \mathbb{C} \mid t=0\} \equiv\{0\}
$$

It has a fundamental solution

$$
Y(z, t)=\left[\begin{array}{cc}
1 & 0 \\
w(z, t) & 1
\end{array}\right]\left(\begin{array}{cc}
z & 0 \\
0 & z^{2} e^{t z}
\end{array}\right)
$$

with

$$
w(z, t):=t^{2} z e^{t z} \operatorname{Ei}(t z)-t \sim \sum_{k=1}^{\infty} \frac{(-1)^{k} k !}{t^{k-1}} z^{-k}, \quad z \rightarrow \infty, \quad-3 \pi / 2<\arg (t z)<3 \pi / 2 .
$$

The above solution has asymptotic representation (5.10), namely (1.6). Now, $t=0$ is a branch point of logarithmic type, since $\operatorname{Ei}(z t)=-\ln (z t)+$ holomorphic function of $z t$. Moreover, the coefficients $F_{k}(t)$ diverge when $t \rightarrow 0$. The reader can check that the system has also fundamental solutions which are holomorphic at $t=0$, but without the standard asymptotic representation $Y_{F}(z, t)$. We also notice a peculiarity of this particular example, namely that $Y(z, t)$ and $Y\left(z e^{-2 \pi i}, t\right)$ are connected by a Stokes matrix $\mathbb{S}=\left[\begin{array}{cc}1 & 0 \\ 2 \pi i t^{2} & 1\end{array}\right]$, which is holomorphic also at $t=0$ and coincides with the trivial Stokes matrix $I$ of the system $\widehat{A}(z, t=0)$.

5.2. Fundamental Solution in a neighbourhood of $t_{\Delta} \in \Delta$, with Assumption 1. Let Assumption 1 hold. Let $t_{\Delta} \in \Delta$. Since the case $t_{\Delta}=0$ has already been discussed in detail, suppose that $t_{\Delta} \neq 0$. Then $t_{\Delta} \in \Delta_{i}$, for some $i \in\{1,2, \ldots, s\}$.

Directions $\tau_{\sigma}^{\left(t_{\Delta}\right)}, \sigma \in \mathbb{Z}$, and sectors $\mathcal{S}_{\sigma}^{\left(t_{\Delta}\right)}$ have been defined in section 5.1 (just put $t_{*}=t_{\Delta}$ ). We leave to the reader the task to adjust the statement of Theorem 2.1 reformulated in a neighbourhood of $t_{\Delta}$, with the block partition of $\Lambda\left(t_{\Delta}\right)$, which is finer than that of $\Lambda(0)$. The closed sector in the theorem will be denoted $\bar{S}^{\left(t_{\Delta}\right)}(\alpha, \beta) \subset \mathcal{S}_{\sigma}^{\left(t_{\Delta}\right)}$. A solution analogous to (4.13) is constructed at $t=t_{\Delta}$, with finer block partition than (4.13). Special cases as in Section 4.2 are very important for us, hence we state the following.

Proposition 4.2 generalized at $t_{\Delta}$ : For $t=t_{\Delta}$, the fundamental solution analogous to (4.13) reduces to an analogous to $(4.20)$, namely

$$
\begin{aligned}
& Y_{\left(t_{\Delta}\right)}(z)=G_{0}\left(t_{\Delta}\right) \mathcal{G}_{\left(t_{\Delta}\right)}(z) z^{B_{1}\left(t_{\Delta}\right)} e^{\Lambda\left(t_{\Delta}\right) z}, \quad \text { with } \quad B_{1}\left(t_{\Delta}\right)=\operatorname{diag}\left(\widehat{A}_{1}\left(t_{\Delta}\right)\right), \\
& \mathcal{G}_{\left(t_{\Delta}\right)}(z) \sim I+\sum_{k=1}^{\infty} F_{\left(t_{\Delta}\right) ; k} z^{-k}, \quad z \rightarrow \infty \quad \text { in } \bar{S}^{\left(t_{\Delta}\right)}(\alpha, \beta),
\end{aligned}
$$

if and only if the following conditions generalising (4.23) hold. For those $a \neq b \in\{1, \ldots, n\}$ such that $u_{a}\left(t_{\Delta}\right)=u_{b}\left(t_{\Delta}\right)$,

$$
\left(\widehat{A}_{1}\left(t_{\Delta}\right)\right)_{a b}=0
$$

and if also $\left(\widehat{A}_{1}\left(t_{\Delta}\right)\right)_{a a}-\left(\widehat{A}_{1}\left(t_{\Delta}\right)\right)_{b b}+l-1=0$ for some $l \geq 2$, the following further conditions must hold:

$$
\begin{aligned}
& \sum_{\{1, \ldots, n\},}\left(\widehat{A}_{1}\left(t_{\Delta}\right)\right)_{a \gamma}\left(F_{\left(t_{\Delta}\right) ; l-1}\right)_{\gamma b}+\sum_{j=1}^{l-2}\left(\widehat{A}_{l-j}\left(t_{\Delta}\right) F_{\left(t_{\Delta}\right) ; j}\right)_{a b}+\left(\widehat{A}_{l}\left(t_{\Delta}\right)\right)_{a b}=0 . \\
& u_{\gamma}\left(t_{\Delta}\right) \neq\left(u_{a}\left(t_{\Delta}\right)=u_{b}\left(t_{\Delta}\right)\right)
\end{aligned}
$$

In the notation used here, then $\stackrel{\circ}{Y}(z)$ in $(4.20)$ is $Y_{(0)}(z)$, while $\mathcal{G}(z)$ in $(4.21)$ is $\mathcal{G}_{(0)}(z)$. Finally, $\stackrel{\circ}{F}_{k}$ in (4.14) is $F_{(0) ; k}$. Keeping into account that $\left(\widehat{A}_{1}\right)_{a \gamma}$ vanishes in (5.12) for $t \rightarrow t_{\Delta}$ and $u_{\gamma}\left(t_{\Delta}\right)=u_{a}\left(t_{\Delta}\right)=$ $u_{b}\left(t_{\Delta}\right)$, it is immediate to prove the following, 
Proposition 5.2 generalised: If the vanishing conditions for (5.11) and (5.12) of Proposition 5.1 hold for $t \rightarrow t_{\Delta} \in \Delta$, then (5.15) and (5.16) at $t=t_{\Delta}$ are satisfied with the choice

$$
F_{\left(t_{\Delta}\right) ; k}=F_{k}\left(t_{\Delta}\right), \quad k \geq 1 .
$$

If moreover $\left(\widehat{A}_{1}\left(t_{\Delta}\right)\right)_{a a}-\left(\widehat{A}_{1}\left(t_{\Delta}\right)\right)_{b b}+l-1 \neq 0$ for every $l \geq 2$, the above (5.17) is the unique choice. Namely, for the system with $t=t_{\Delta}$ there is only the unique formal solution

$$
\left(I+\sum_{k=1}^{\infty} F_{k}\left(t_{\Delta}\right)\right) z^{B_{1}\left(t_{\Delta}\right)} z^{\Lambda\left(t_{\Delta}\right)}, \quad B_{1}\left(t_{\Delta}\right)=\operatorname{diag}\left(\widehat{A}_{1}\left(t_{\Delta}\right)\right) .
$$

\section{PART II: Stokes Phenomenon}

When Assumption 1 holds, the system (2.1) is gauge equivalent to (2.15) (i.e. system (1.5) in the Introduction) with $G_{0}(t)$ diagonalizing $A_{0}(t)$, namely

$$
\frac{d \widehat{Y}}{d z}=\widehat{A}(z, t) \widehat{Y}, \quad \widehat{A}(z, t):=G_{0}^{-1}(t) A(z, t) G_{0}(t)=\Lambda(t)+\sum_{k=1}^{\infty} \widehat{A}_{k}(t) z^{-k} .
$$

At $t_{0} \notin \Delta, \Lambda\left(t_{0}\right)$ has distinct eigenvalues, the Stokes phenomenon is studied as in [2]. We describe below the analogous results at $t=0$ and $t_{\Delta} \in \Delta$, namely the existence and uniqueness of fundamental solutions with given asymptotics (4.14) in wide sectors. The results could be derived from the general construction of [4], especially from Theorem V and VI therein ${ }^{15}$. Nevertheless, it seems to be more natural to us to derive them in straightforward way, which we present below. First, we concentrate on the most degenerate case $\Lambda=\Lambda(0)$, for $t=0$, so that $A(z, 0)=\widehat{A}(z, 0)$ and the systems (2.1) and (5.18) coincide. In Section 7 we consider the case of any other $t_{\Delta} \in \Delta$.

\section{Stokes Phenomenon at $t=0$}

6.1. Stokes Rays of $\Lambda=\Lambda(0)$.

Definition 6.1. The Stokes rays associated with the pair of eigenvalues $\left(\lambda_{j}, \lambda_{k}\right), 1 \leq j \neq k \leq n$, of $\Lambda$ are the infinitely many rays contained in the universal covering $\mathcal{R}$ of $\mathbb{C} \backslash\{0\}$, oriented outwards from 0 to $\infty$, defined by

$$
\Re\left(\left(\lambda_{j}-\lambda_{k}\right) z\right)=0, \quad \Im\left(\left(\lambda_{j}-\lambda_{k}\right) z\right)<0, \quad z \in \mathcal{R} .
$$

The definition above implies that for a couple of eigenvalues $\left(\lambda_{j}, \lambda_{k}\right)$ the associated rays are

$$
R\left(\theta_{j k}+2 \pi N\right):=\left\{z \in \mathcal{R} \mid z=\rho e^{i\left(\theta_{j k}+2 \pi N\right)}, \quad \rho>0\right\}, \quad N \in \mathbb{Z} .
$$

where

$$
\theta_{j k}:=\frac{3 \pi}{2}-\arg _{p}\left(\lambda_{j}-\lambda_{k}\right) .
$$

- Labelling: We enumerate Stokes rays with $\nu \in \mathbb{Z}$, using directions $\tau_{\nu}$ introduced in Section 2. Indeed, by Definition 6.1, Stokes rays have directions $\arg z=\tau_{\nu}$, ordered in counter-clockwise sense as $\nu$ increases. For any sector of central angle $\pi$ in $\mathcal{R}$, whose boundaries are not Stokes rays, there exists a $\nu_{0} \in \mathbb{Z}$ such that the $\mu$ Stokes rays $\tau_{\nu_{0}-\mu+1}<\cdots<\tau_{\nu_{0}-1}<\tau_{\nu_{0}}$ are contained in the sector. All other Stokes rays have directions

$$
\arg z=\tau_{\nu+k \mu}:=\tau_{\nu}+k \pi, \quad k \in \mathbb{Z}, \quad \nu \in\left\{\nu_{0}-\mu+1, \ldots, \nu_{0}-1, \nu_{0}\right\} .
$$

Rays $\tau_{\nu_{0}-\mu+1}<\cdots<\tau_{\nu_{0}-1}<\tau_{\nu_{0}}$ are called a set of basic Stokes rays, because they generate the others ${ }^{16}$.

\footnotetext{
${ }^{15}$ Note that notations here and in [4] are similar, but they indicate objects that are slightly different (for example Stokes rays $\tau_{\nu}$ and sectors $\mathcal{S}_{\nu}$ are not defined in the same way).

${ }^{16}$ Although notations are similar to [4], definitions are slightly different here.
} 
- Sectors $\mathcal{S}_{\nu}$ : Consider a sector $S$ of central opening less than $\pi$, with boundary rays which are not Stokes rays. The first rays encountered outside $S$ upon moving clockwise and anti-clockwise, will be called the two nearest Stokes rays outside $S$. If $S$ contains in its interior a set of basic rays, say $\tau_{\nu+1-\mu}, \tau_{\nu+2-\mu}, \ldots, \tau_{\nu}$, then the two nearest Stokes rays outside $S$ are $\tau_{\nu-\mu}$ and $\tau_{\nu+1}$, namely the boundaries rays of $\mathcal{S}_{\nu}$ in (2.12), and obviously $S \subset \mathcal{S}_{\nu}$.

- Projections onto $\mathbb{C}$ : If $R$ is any of the rays in $\mathcal{R}$, its projection onto $\mathbb{C}$ will be denoted $P R$. For example, let $\bar{\lambda}_{j}$ be the complex conjugate of $\lambda_{j}$, then for any $N$ the projection of (6.1) is

$$
P R\left(\theta_{j k}+2 \pi N\right)=\left\{z \in \mathbb{C} \mid z=-i \rho\left(\bar{\lambda}_{j}-\bar{\lambda}_{k}\right), \rho>0\right\} .
$$

Definition 6.2. An admissible ray for $\Lambda(0)$ is a ray $R(\widetilde{\tau}):=\left\{z \in \mathcal{R} \mid z=\rho e^{i \widetilde{\tau}}, \quad \rho>0\right\}$ in $\mathcal{R}$, of direction $\widetilde{\tau} \in \mathbb{R}$, which does not coincide with any of the Stokes rays of $\Lambda(0)$. Let

$$
\begin{aligned}
& l_{+}(\widetilde{\tau}):=P R(\widetilde{\tau}+2 k \pi), \quad l_{-}(\widetilde{\tau}):=P R(\widetilde{\tau}+(2 k+1) \pi), \quad k \in \mathbb{Z}, \\
& l(\widetilde{\tau}):=l_{-}(\widetilde{\tau}) \cup\{0\} \cup l_{+}(\widetilde{\tau}) .
\end{aligned}
$$

We call the oriented line $l(\widetilde{\tau})$ an admissible line for $\Lambda(0)$. Its positive part is $l_{+}(\widetilde{\tau})$.

Observe that there exists a suitable $\nu$ such that $\tau_{\nu}<\widetilde{\tau}<\tau_{\nu+1}$, which implies

$$
R(\widetilde{\tau}) \subset \mathcal{S}_{\nu} \cap \mathcal{S}_{\nu+\mu}, \quad R(\widetilde{\tau}+\pi) \subset \mathcal{S}_{\nu+\mu} \cap \mathcal{S}_{\nu+2 \mu} .
$$

In particular, if $\tau$ is as in (2.10), then $\tau_{-1}<\tau<\tau_{0}$, and $l(\tau)$ is an admissible line.

6.2. Uniqueness of the Fundamental Solution with given Asymptotics. In case of distinct eigenvalues, it is well known that there exists a unique fundamental solution, determined by the asymptotic behaviour given by the formal solution, on a sufficiently large sector. This fact must now be proved also at coalescence points.

Let the diagonal form $\Lambda=\Lambda_{1} \oplus \cdots \oplus \Lambda_{s}$ of $A_{0}$ be fixed. Let a formal solution $\stackrel{\circ}{Y}_{F}(z)=\Delta_{0} F(z) z^{D} z^{L} e^{\Lambda z}$ be chosen in the class of formal solutions with given $\Delta_{0}, D, L, \Lambda$, as in Definition 4.1. As a consequence of Theorem 2.1 and Theorem 4.1, there exists at least one actual solution as in (4.13), namely

$$
\stackrel{\circ}{Y}(z)=\Delta_{0} \mathcal{G}(z) z^{D} z^{L} e^{\Lambda z}, \quad \mathcal{G}(z) \sim F(z), \quad z \rightarrow \infty, \quad z \in \bar{S}(\alpha, \beta) .
$$

Observe that $\bar{S}(\alpha, \beta)$ can be chosen in Theorem 2.1 so that it contains the set of basic Stokes rays of $\mathcal{S}_{\nu}$, namely $\tau_{\nu+1-\mu}, \ldots, \tau_{\nu-1}, \tau_{\nu}$. The asymptotic relation in (6.4) is conventionally written as follows,

$$
\stackrel{\circ}{Y}(z) \sim \stackrel{\circ}{Y}_{F}(z), \quad z \rightarrow \infty, \quad z \in \bar{S}(\alpha, \beta) .
$$

Now, $\mathcal{G}(z)$ is holomorphic for $|z|$ sufficiently big in $\bar{S}(\alpha, \beta)$. Since $A(z)$ has no singularities for $|z| \geq N_{0}$ large, except the point at infinity, then $\stackrel{\wp}{Y}(z)$ and $\mathcal{G}(z)$ have analytic continuation on $\mathcal{R} \cap\left\{|z| \geq N_{0}\right\}$.

Lemma 6.1. Let $C \in G L(n, \mathbb{C})$, and $S$ an arbitrary sector. Then

$$
z^{D} z^{L} C z^{-L} z^{-D} \sim I, \quad z \rightarrow \infty \text { in } S \Longleftrightarrow z^{D} z^{L} C z^{-L} z^{-D}=I \quad \Longleftrightarrow \quad C=I .
$$

The simple proof is left as an exercise.

Lemma 6.2 (Extension Lemma). Let $\stackrel{\circ}{Y}(z)$ be a fundamental matrix solution with asymptotic behaviour,

$$
\stackrel{\circ}{Y}(z) \sim \stackrel{\circ}{Y}_{F}(z), \quad z \rightarrow \infty, \quad z \in S,
$$

in a sector $S$ of a non specified central opening angle. Suppose that there is a sector $\widetilde{S}$ not containing Stokes rays, such that $S \cap \widetilde{S} \neq \emptyset$. Then,

$$
\stackrel{\circ}{Y}(z) \sim \stackrel{\circ}{Y}_{F}(z), \quad z \rightarrow \infty, \quad \text { for } z \in S \cup \tilde{S} .
$$

Proof: $\widetilde{S}$ has central opening angle less than $\pi$, because it does not contain Stokes rays. Therefore, by Theorem 2.1, there exists a fundamental matrix solution $\tilde{Y}(z)=\Delta_{0} \tilde{\mathcal{G}}(z) z^{D} z^{L} e^{\Lambda z}$, with asymptotic behaviour $\widetilde{Y}(z) \sim \stackrel{\circ}{F}_{F}(z)$, for $z \rightarrow \infty, z \in \widetilde{S}$. The two fundamental matrices are connected by an invertible matrix $C$, namely ${ }_{Y}(z)=\widetilde{Y}(z) C, z \in S \cap \widetilde{S}$. Therefore,

$$
\widetilde{\mathcal{G}}^{-1}(z) \mathcal{G}(z)=z^{D} z^{L} e^{\Lambda z} C e^{-\Lambda z} z^{-L} z^{-D} .
$$


Since $\mathcal{G}(z)$ and $\widetilde{\mathcal{G}}^{-1}(z)$ have the same asymptotic behaviour in $S \cap \widetilde{S}$, the l.h.s has asymptotic series equal to the identity matrix $I$, for $z \rightarrow \infty$ in $z \in S \cap \widetilde{S}$. Thus, so must hold for the r.h.s. The r.h.s has diagonal-block structure inherited from $\Lambda$. We write the block $[i, j], 1 \leq i, j \leq s$, of $C$ with simple notation $C_{i j}$. The block $[i, j]$ in r.h.s. is then, $e^{\left(\lambda_{i}-\lambda_{j}\right) z} z^{D_{i}} z^{L_{i}} C_{i j} z^{-L_{j}} z^{-D_{j}}$. Hence, the following must hold,

$$
e^{\left(\lambda_{i}-\lambda_{j}\right) z} z^{D_{i}} z^{L_{i}} C_{i j} z^{-L_{j}} z^{-D_{j}} \sim \delta_{i j} I_{i}, \quad z \rightarrow \infty, \quad z \in S \cap \widetilde{S}
$$

Here $I_{i}$ is the $p_{i} \times p_{i}$ identity matrix.

- For $i \neq j$ : Since there are no Stokes rays in $\widetilde{S}$, the sign of $\Re\left(\lambda_{i}-\lambda_{j}\right) z$ does not change in $\widetilde{S}$. This implies that $e^{\left(\lambda_{i}-\lambda_{j}\right) z} z^{D_{i}} z^{L_{i}} C_{i j} z^{-L_{j}} z^{-D_{j}} \sim 0$ for $z \rightarrow \infty$ in $\widetilde{S}$.

- For $i=j$ : We have $z^{D_{i}} z^{L_{i}} C_{i i} z^{-L_{i}} z^{-D_{i}} \sim I_{i}$ for $z \rightarrow \infty$ in $S \cap \widetilde{S}$. From Lemma 6.1 it follows that $z^{D_{i}} z^{L_{i}} C_{i i} z^{-L_{i}} z^{-D_{i}}=I_{i}$. This holds on the whole $\widetilde{S}$.

The above considerations imply that $z^{D} z^{L} e^{\Lambda z} C e^{-\Lambda z} z^{-L} z^{-D} \sim I$ for $z \rightarrow \infty$ in $\widetilde{S}$. From the fact that $\widetilde{\mathcal{G}}(z) \sim I+\sum_{k \geq 1} \stackrel{\circ}{F}_{k} z^{-k}$ in $\widetilde{S}$, we conclude that also $\mathcal{G}(z) \sim I+\sum_{k \geq 1} \stackrel{\circ}{F}_{k} z^{-k}$ for $z \rightarrow \infty$ in $\widetilde{S}$. Therefore, $\mathcal{G}(z) \sim I+\sum_{k \geq 1} \stackrel{\circ}{F}_{k} z^{-k}$ in $S \cup \widetilde{S}$.

The extension Lemma immediately implies the following:

Theorem 6.1 (Extension Theorem). Let $Y$ $(z)$ be a fundamental matrix solution such that ${ }_{Y}(z) \sim \dot{Y}_{F}(z)$ in a sector $S$, containing a set of $\mu$ basic Stokes rays, and no other Stokes rays. Then, the asymptotics $\stackrel{\circ}{Y}(z) \sim \stackrel{\circ}{Y}_{F}(z)$ holds on the open sector which extends up to the two nearest Stokes rays outside S. This sector has central opening angle greater than $\pi$ and is a sector $\mathcal{S}_{\nu}$ for a suitable $\nu$.

Important Remark: The above extension theorem has the important consequence that in the statement of Theorem 4.1 and Proposition 4.2, the matrix $\mathcal{G}(z)$, which has analytic continuation in $\mathcal{R}$ for $|z| \geq N_{0}$, has the prescribed asymptotic expansion in any proper closed subsector of $\mathcal{S}_{\nu}$. Hence, by definition, the asymptotics holds in the open sector $\mathcal{S}_{\nu}$.

Theorem 6.2 (Uniqueness Theorem). A fundamental matrix ${ }_{Y} Y(z)$ as (4.13) such that ${ }_{Y}(z) \sim \dot{\circ}_{F}(z)$, for $z \rightarrow \infty$ in a sector $S$ containing a set of basic Stokes rays, is unique. In particular, this applies if $\bar{S}(\alpha, \beta)$ of Theorem 2.1 contains a set of basic Stokes rays.

Proof: Suppose that there are two solutions ${ }^{\circ}(z)$ and $\tilde{Y}(z)$ with asymptotic representation $\stackrel{\circ}{Y}_{F}(z)$ in a sector $S$, which contains $\mu$ basic Stokes rays. Then, there exists an invertible matrix $C$ such that $\stackrel{\circ}{Y}(z)=\tilde{Y}(z) C$, namely

$$
\widetilde{\mathcal{G}}^{-1}(z) \mathcal{G}(z)=z^{D} z^{L} e^{\Lambda z} C e^{-\Lambda z} z^{-L} z^{-D} .
$$

The l.h.s. has asymptotic series equal to $I$ as $z \rightarrow \infty$ in $S$. Therefore, for the block $[i, j]$, the following must hold,

$$
e^{\left(\lambda_{i}-\lambda_{j}\right) z} z^{D_{i}} z^{L_{i}} C_{i j} z^{-L_{j}} z^{-D_{j}} \sim \delta_{i j} I_{i}, \quad \text { for } z \rightarrow \infty \text { in } S .
$$

Since $S$ contains a set of basic Stokes rays, $\Re\left(\lambda_{i}-\lambda_{j}\right) z$ changes sign at least once in $S$, for any $1 \leq i \neq$ $j \leq s$. Thus, $e^{\left(\lambda_{i}-\lambda_{j}\right) z}$ diverges in some subsector of $S$. For $i \neq j$ this requires that $C_{i j}=0$ for $i \neq j$. For $i=j$, we have $z^{D_{i}} z^{L_{i}} C_{i i} z^{-L_{i}} z^{-D_{i}} \sim I_{i}$. Lemma 6.1 assures that $C_{i i}=I_{i}$. Thus, $C=I$.

- [The notation $Y_{\nu}(z)$ ]: There exist $\nu \in \mathbb{Z}$ such that a sector $S$ of Theorem 6.2 contains the basic rays $\tau_{\nu+1-\mu}, \ldots, \tau_{\nu-1}, \tau_{\nu}$. Hence $S \subset \mathcal{S}_{\nu}$. The unique fundamental solution of Theorem 6.2, with asymptotics extended to $\mathcal{S}_{\nu}$ according to Theorem 6.1 , will be denoted $\stackrel{\circ}{Y}_{\nu}(z)$. 
6.3. Stokes Matrices. The definition of Stokes matrices is standard. Recall that the Stokes rays associated with $\left(\lambda_{j}, \lambda_{k}\right)$ are (6.1). Consider also the rays

$$
R\left(\theta_{j k}+2 \pi N+\delta\right)=\left\{z \in \mathcal{R} \mid z=\rho e^{i\left(\theta_{j k}+2 \pi N+\delta\right)}, \quad \rho>0\right\}, \quad N \in \mathbb{Z} .
$$

The sign of $\Re\left(\lambda_{j}-\lambda_{k}\right) z$ for $z \in R_{N}\left(\theta_{j k}+\delta\right)$ is:

$$
\left\{\begin{array}{cccc}
\Re\left(\lambda_{j}-\lambda_{k}\right) z<0, & \text { for } & -\pi<\delta<0 & \bmod 2 \pi \\
\Re\left(\lambda_{j}-\lambda_{k}\right) z>0, & \text { for } & 0<\delta<\pi & \bmod 2 \pi \\
\Re\left(\lambda_{j}-\lambda_{k}\right) z=0, & \text { for } & \delta=0, \pi,-\pi & \bmod 2 \pi
\end{array}\right.
$$

Definition 6.3 (Dominance relation). In a sector where $\Re\left(\lambda_{j}-\lambda_{k}\right) z>0, \lambda_{j}$ is said to be dominant over $\lambda_{k}$ in that sector, and we write $\lambda_{j} \succ \lambda_{k}$. In a sector where $\Re\left(\lambda_{j}-\lambda_{k}\right) z<0$, $\lambda_{j}$ is said to be sub-dominant, or dominated by $\lambda_{k}$, and we write $\lambda_{j} \prec \lambda_{k}$.

If a sector $S$ does not contain Stokes rays in its interior, it is well defined a dominance relation in $S$, which determines an ordering relation among eigenvalues, referred to the sector $S$.

Denote by

$$
\stackrel{\circ}{Y}_{\nu}(z) \text { and } \stackrel{\circ}{Y}_{\nu+\mu}(z)
$$

the unique fundamental solutions (4.13) with asymptotic behaviours $\stackrel{\circ}{F}_{F}(z)$ on $\mathcal{S}_{\nu}$ and $\mathcal{S}_{\nu+\mu}$ respectively, as in Theorem 6.2. Observe that $\mathcal{S}_{\nu} \cap \mathcal{S}_{\nu+\mu}=S\left(\tau_{\nu}, \tau_{\nu+1}\right)$ is not empty and does not contain Stokes rays.

Definition 6.4. For any $\nu \in \mathbb{Z}$, the Stokes matrix $\stackrel{S}{S}_{\nu}$ is the connection matrix such that

$$
\stackrel{\circ}{Y}_{\nu+\mu}(z)=\stackrel{\circ}{Y}_{\nu}(z) \stackrel{\circ}{S}_{\nu}, \quad z \in \mathcal{R} .
$$

Proposition 6.1. Let $\prec$ be the dominance relation referred to the sector $\mathcal{S}_{\nu} \cap \mathcal{S}_{\nu+\mu}$. Then, the Stokes matrix $\stackrel{\circ}{\mathbb{S}}_{\nu}$ has the following block-triangular structure:

$$
\begin{aligned}
& \stackrel{\mathbb{S}}{j j}_{j}^{(\nu)}=I_{p_{j}}, \\
& \stackrel{\mathbb{S}}{j k}^{(\nu)}=0 \text { for } \lambda_{j} \succ \lambda_{k} \text { in } \mathcal{S}_{\nu} \cap \mathcal{S}_{\nu+\mu}, \quad j, k \in\{1,2, \ldots, s\} .
\end{aligned}
$$

Proof: We re-write (6.5) as,

$$
\mathcal{G}_{\nu}^{-1}(z) \mathcal{G}_{\nu+\mu}(z)=z^{D} z^{L} e^{\Lambda z} \stackrel{\circ}{S}_{\nu} e^{-\Lambda z} z^{-L} z^{-D}
$$

For $z \in \mathcal{S}_{\nu} \cap \mathcal{S}_{\nu+\mu}$, the l.h.s. has asymptotic expansion equal to $I$. Hence, the same must hold for the r.h.s. Recalling that no Stokes rays lie in $\mathcal{S}_{\nu} \cap \mathcal{S}_{\nu+\mu}$, we find:

- For $j \neq k$, we have $e^{\left(\lambda_{j}-\lambda_{k}\right) z} z^{D_{j}} z^{L_{j}} \stackrel{\circ}{\mathbb{S}}_{j k}^{(\nu)} z^{-L_{k}} z^{-D_{k}} \sim 0$ in $\mathcal{S}_{\nu} \cap \mathcal{S}_{\nu+\mu}$ if and only if $\dot{\mathbb{S}}_{j k}^{(\nu)}=0$ for $\lambda_{j} \succ \lambda_{k}$, where the dominance relation is referred to the sector $\mathcal{S}_{\nu} \cap \mathcal{S}_{\nu+\mu}$.

- For $j=k$, we have $z^{D_{j}} z^{L_{j}} \stackrel{\leftrightarrow}{S}_{j j}^{(\nu)} z^{-L_{j}} z^{-D_{j}} \sim I_{p_{j}}$ if and only if $\stackrel{\mathbb{S}}{j j}_{j}^{(\nu)}=I_{p_{j}}$, by Lemma 6.1. This proves the Proposition.

6.4. Canonical Sectors, Complete Set of Stokes Matrices, Monodromy Data. There are no Stokes rays in the intersection of successive sectors $\mathcal{S}_{\nu+k \mu}$ and $\mathcal{S}_{\nu+(k+1) \mu}$ (recall that $\tau_{\nu}+k \pi=\tau_{\nu+k \mu}$ for any $k \in \mathbb{Z}$ ). Therefore, we can introduce the unique fundamental matrix solutions

$$
\stackrel{\circ}{Y}_{\nu+k \mu}(z)
$$

with asymptotic behaviour $\stackrel{\circ}{Y}_{F}(z)$ in $\mathcal{S}_{\nu+k \mu}$, and the Stokes matrices $\stackrel{\circ}{\mathbb{S}}_{\nu+k \mu}$ connecting them,

$$
\stackrel{\circ}{Y}_{\nu+(k+1) \mu}(z)=\stackrel{\circ}{Y}_{\nu+k \mu}(z) \stackrel{\circ}{\mathbb{S}}_{\nu+k \mu}, \quad z \in \mathcal{R} .
$$

From Proposition 6.1 , it follows that the blocks $[j, k]$ and $[k, j]$ satisfy

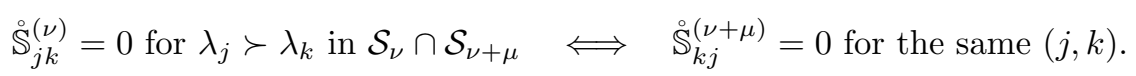

We call $\mathcal{S}_{\nu}, \mathcal{S}_{\nu+\mu}, \mathcal{S}_{\nu+2 \mu}$ the canonical sectors associated with $\tau_{\nu}$.

Given a formal solution, a simple computation (recall that $[L, \Lambda]=0)$ yields $\stackrel{\circ}{Y}_{F}\left(e^{2 \pi i} z\right)=\stackrel{\circ}{Y}_{F}(z) e^{2 \pi i L}$. $L$ is called exponent of formal monodromy. 
Theorem 6.3. We introduce the notation $z_{(\nu)}$ if $z \in \mathcal{S}_{\nu}$. Thus $z_{(\nu+2 \mu)}=e^{2 \pi i} z_{(\nu)}$. The following equalities hold

$$
\begin{aligned}
& \text { (i) } \stackrel{\circ}{Y}_{\nu+2 \mu}\left(z_{(\nu+2 \mu)}\right)=\stackrel{\circ}{Y}_{\nu}\left(z_{(\nu)}\right) e^{2 \pi i L} \text {, } \\
& \text { (ii) } \stackrel{\circ}{Y}_{\nu+2 \mu}(z)=\check{\Upsilon}_{\nu}(z) \stackrel{\circ}{\mathbb{S}}_{\nu} \stackrel{\circ}{\mathbb{S}}_{\nu+\mu}, \quad z \in \mathcal{R}, \\
& \text { (iii) } \stackrel{\circ}{Y}_{\nu}\left(e^{2 \pi i} z\right)=\stackrel{\circ}{Y}_{\nu}(z) e^{2 \pi i L}\left(\stackrel{\circ}{S}_{\nu} \stackrel{\circ}{S}_{\nu+\mu}\right)^{-1}, \quad z \in \mathcal{R} .
\end{aligned}
$$

where $|z| \geq N_{0}$ is sufficiently large, in such a way that any other singularity of $A(z)$ is contained in the ball $|z|<N_{0}$.

Proof: As in the case of distinct eigenvalues. Alternatively, one can adapt Proposition 4 of [4] to the present case. ${ }^{17}$.

The equality (iii) provides the monodromy matrix $M_{\infty}^{(\nu)}$ of $\dot{Y}_{\nu}(z)$ at $z=\infty$ :

$$
M_{\infty}^{(\nu)}:=\left(\dot{\mathbb{S}}_{\nu} \stackrel{\circ}{S}_{\nu+\mu}\right) e^{-2 \pi i L} .
$$

corresponding to a clockwise loop with $|z| \geq N_{0}$ large, in such a way that all other singularities of $A(z)$ are inside the loop.

The two Stokes matrices $\stackrel{\AA}{\mathbb{S}}_{\nu}, \stackrel{\circ}{\mathbb{S}}_{\nu+\mu}$, and the matrix $L$ generate all the other Stokes matrices $\stackrel{\circ}{\mathbb{S}}_{\nu+k \mu}$, according to the following proposition

Proposition 6.2. For any $\nu \in \mathbb{Z}$, the following holds: $\stackrel{\circ}{S}_{\nu+2 \mu}=e^{-2 \pi i L} \stackrel{\circ}{\mathbb{S}}_{\nu} e^{2 \pi i L}$.

Proof: For simplicity, take $\nu=0$. A point in $z \in \mathcal{S}_{2 \mu} \cap \mathcal{S}_{3 \mu}$ can represented both as $z_{(2 \mu)}$ and $z_{(3 \mu)}$, and a point in $\mathcal{S}_{0} \cap \mathcal{S}_{\mu}$ is represented both as $z_{(0)}$ and $z_{(\mu)}$. Therefore, the l.h.s. of the equality $\stackrel{\circ}{Y}_{3 \mu}(z)=\dot{Y}_{2 \mu}(z) \stackrel{\circ}{\mathbb{S}}_{2 \mu}$ is $\stackrel{\circ}{Y}_{3 \mu}\left(z_{(3 \mu)}\right)=\dot{Y}_{\mu}\left(z_{(\mu)}\right) e^{2 \pi i L}=\stackrel{\circ}{Y}_{0}\left(z_{(0)}\right) \stackrel{\circ}{\mathbb{S}}_{0} e^{2 \pi i L}$. The r.h.s. is $\dot{Y}_{2 \mu}\left(z_{(2 \mu)}\right) \stackrel{\mathbb{S}}{2 \mu}_{2 \mu}=$ $\stackrel{\circ}{Y}_{0}\left(z_{(0)}\right) e^{2 \pi i L} \quad \stackrel{\circ}{\mathbb{S}}_{2 \mu}$. Thus $\dot{\circ}_{0}\left(z_{(0)}\right) \stackrel{\leftrightarrow}{\mathbb{S}}_{0} e^{2 \pi i L}=\stackrel{\circ}{0}_{0}\left(z_{(0)}\right) e^{2 \pi i L} \stackrel{\circ}{\mathbb{S}}_{2 \mu}$. This proves the proposition.

The above proposition implies that $\stackrel{\mathscr{S}}{\nu+k \mu}_{\nu}$ are generated by $\stackrel{\mathbb{S}}{\nu}_{\nu}, \stackrel{\mathscr{S}}{\nu+\mu}_{\nu}$, which therefore form a complete set of Stokes matrices. A complete set of Stokes matrices and the exponent of formal monodromy are necessary and sufficient to obtain the monodromy at $z=\infty$, through formula (6.7). This justifies the following definition.

Definition 6.5. For a chosen $\nu,\left\{\stackrel{\mathbb{S}}{\nu}_{\nu}, \stackrel{\mathbb{S}}{\nu+\mu}_{\nu}, L\right\}$ is a set of monodromy data at $z=\infty$ of the system (2.1) with $t=0$.

Remark 6.1. By a factorization into Stokes factors, as in the proof of Theorem 15.1 below, it can

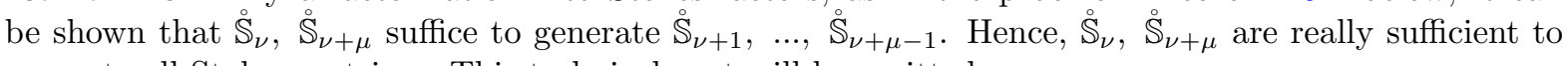
generate all Stokes matrices. This technical part will be omitted.

\section{Stokes Phenomenon at fixed $t_{\Delta} \in \Delta$}

The results of Section 6 apply to any other $t_{\Delta} \in \Delta$. By a permutation matrix $P$ we arrange $P^{-1} \Lambda\left(t_{\Delta}\right) P$ in blocks, in such a way that each block has only one eigenvalue and two distinct blocks have different eigenvalues. This is achieved by the transformation $\widehat{Y}(z, t)=P \widetilde{Y}(z, t)$ applied to the system (5.18). Then, the procedure is exactly the same of Section 6, applied to the system

$$
\frac{d \widetilde{Y}}{d z}=P^{-1} \widehat{A}\left(z, t_{\Delta}\right) P \tilde{Y}
$$

\footnotetext{
${ }^{17}$ With the warning that notations are similar but objects are slightly different here and in [4].
} 
The block partition of all matrices in the computations and statements is that inherited from $P^{-1} \Lambda\left(t_{\Delta}\right) P$. The Stokes rays are defined in the same way as in Definition 6.1, using the eigenvalues of $\Lambda\left(t_{\Delta}\right)$, namely

$$
\begin{aligned}
& \Re\left(\left(u_{a}\left(t_{\Delta}\right)-u_{b}\left(t_{\Delta}\right)\right) z\right)=0, \quad \Im\left(\left(u_{a}\left(t_{\Delta}\right)-u_{a}\left(t_{\Delta}\right)\right) z\right)<0, \quad z \in \mathcal{R}, \\
& \text { for } 1 \leq a \neq b \leq n \quad \underline{\text { and }} \quad u_{a}\left(t_{\Delta}\right) \neq u_{a}\left(t_{\Delta}\right) .
\end{aligned}
$$

Hence, the Stokes rays associated with $u_{a}\left(t_{\Delta}\right), u_{b}\left(t_{\Delta}\right)$ are the infinitely many rays with directions

$$
\arg z=\frac{3 \pi}{2}-\arg _{p}\left(u_{a}\left(t_{\Delta}\right)-u_{b}\left(t_{\Delta}\right)\right)+2 N \pi, \quad N \in \mathbb{Z}
$$

The rays associated with $u_{b}\left(t_{\Delta}\right), u_{a}\left(t_{\Delta}\right)$ are opposite to the above, having directions

$$
\arg z=\frac{3 \pi}{2}-\arg _{p}\left(u_{b}\left(t_{\Delta}\right)-u_{a}\left(t_{\Delta}\right)\right)+2 N \pi
$$

We conclude that all Stokes rays have directions

$$
\arg z=\tau_{\sigma}^{\left(t_{\Delta}\right)}, \quad \sigma \in \mathbb{Z}
$$

analogous to $(6.3)$, with directions $\tau_{\sigma}^{\left(t_{\Delta}\right)}$ defined in Section 5.1. Once the Stokes matrices for the above system are computed, in order to go back to the original arrangement corresponding to $\Lambda\left(t_{\Delta}\right)$ we just apply the inverse permutation. Namely, if $\mathbb{S}$ is a Stokes matrix of $(7.1)$, then $P \mathbb{S} P^{-1}$ is a Stokes matrix for (5.18) with $t=t_{\Delta}$.

\section{Stokes Phenomenon at $t_{0} \notin \Delta$}

The results of Section 6 (extension theorem, uniqueness theorem, Stokes matrices, etc) apply a fortiori if the eigenvalues are distinct, namely at a point $t_{0} \notin \Delta$ such that Theorem 5.1 and Corollary 5.1 apply. The block partition of $\Lambda\left(t_{0}\right)$ is into one-dimensional blocks, being the eigenvalues all distinct, and we are back to the well known case of [2]. The Stokes rays are defined in the same way as in Definition 6.1, using the eigenvalues of $\Lambda\left(t_{0}\right)$, namely

$$
\Re\left(\left(u_{a}\left(t_{0}\right)-u_{b}\left(t_{0}\right)\right) z\right)=0, \quad \Im\left(\left(u_{a}\left(t_{0}\right)-u_{b}\left(t_{0}\right)\right) z\right)<0, \quad z \in \mathcal{R}, \quad \forall 1 \leq a \neq b \leq n .
$$

Since and $u_{a}\left(t_{0}\right) \neq u_{b}\left(t_{0}\right)$ for any $a \neq b$, the above definition holds for any $1 \leq a \neq b \leq n$. Hence, the Stokes rays associated with $u_{a}\left(t_{0}\right), u_{b}\left(t_{0}\right)$ are the infinitely many rays with directions

$$
\arg z=\frac{3 \pi}{2}-\arg _{p}\left(u_{a}\left(t_{0}\right)-u_{b}\left(t_{0}\right)\right)+2 N \pi, \quad N \in \mathbb{Z} .
$$

The rays associated with $u_{b}\left(t_{0}\right), u_{a}\left(t_{0}\right)$ are opposite to the above, having directions

$$
\arg z=\frac{3 \pi}{2}-\arg _{p}\left(u_{b}\left(t_{0}\right)-u_{a}\left(t_{0}\right)\right)+2 N \pi .
$$

We conclude that all Stokes rays have directions

$$
\arg z=\tau_{\sigma}^{\left(t_{0}\right)}, \quad \sigma \in \mathbb{Z}
$$

analogous to $(6.3)$, being the directions $\tau_{\sigma}^{\left(t_{0}\right)}$ defined in Section 5.1. We stress that $t_{0}$ is fixed here. The Stokes phenomenon is studied in the standard way. The canonical sectors are the sectors $\mathcal{S}_{\sigma}^{\left(t_{0}\right)}$ of Theorem 5.1. The sector $\mathcal{S}_{\sigma}^{\left(t_{0}\right)}$ contains the set of basic Stokes rays

$$
\tau_{\sigma+1-\mu_{t_{0}}}^{\left(t_{0}\right)}, \quad \tau_{\sigma+2-\mu_{t_{0}}}^{\left(t_{0}\right)}, \quad \ldots, \quad \tau_{\sigma}^{\left(t_{0}\right)},
$$

which serve to generate all the other rays by adding multiples of $\pi$. The rays $\tau_{\sigma-\mu_{t_{0}}}^{\left(t_{0}\right)}$ and $\tau_{\sigma+1}^{\left(t_{0}\right)}$ are the nearest Stokes rays, boundaries of $\mathcal{S}_{\sigma}^{\left(t_{0}\right)}$. The Stokes matrices connect solutions of Corollary 5.1, having the prescribed canonical asymptotics on successive sectors, for example $\mathcal{S}_{\sigma}^{\left(t_{0}\right)}, \mathcal{S}_{\sigma+\mu_{t_{0}}}^{\left(t_{0}\right)}, \mathcal{S}_{\sigma+2 \mu_{t_{0}}}^{\left(t_{0}\right)}$, etc.

Our purpose is now to show how the Stokes phenomenon can be described in a consistent "holomorphic" way as $t$ varies. The definition of Stokes matrices for varying $t$ will require some steps.

\section{PART III: Cell Decomposition, $t$-analytic Stokes Matrices}




\section{Stokes Rays Rotate as $t$ VARIES}

At $t=0$, Stokes rays have directions $3 \pi / 2-\arg _{p}\left(\lambda_{i}-\lambda_{j}\right)+2 N \pi, 1 \leq i \neq j \leq s$. For $t$ away from $t=0$, the following occurs:

1) [Splitting] For $1 \leq i \neq j \leq s$, there are rays of directions $3 \pi / 2-\arg _{p}\left(u_{a}(t)-u_{b}(t)\right) \bmod (2 \pi)$, with $u_{a}(0)=\lambda_{i}, u_{b}(0)=\lambda_{j}$. These rays are the splitting of $3 \pi / 2-\arg _{p}\left(\lambda_{i}-\lambda_{j}\right) \bmod (2 \pi)$ into more rays.

2) [Unfolding] For any $i=1,2, \ldots, s$, new rays appear, with directions $3 \pi / 2-\arg _{p}\left(u_{a}(t)-u_{b}(t)\right)$, $u_{a}(0)=u_{b}(0)=\lambda_{i}$. These rays are due to the unfolding of $\lambda_{i}$.

The cardinality of a set of basic Stokes rays is maximal away from the coalescence locus $\Delta$, minimal at $t=0$, and intermediate at $t_{\Delta} \in \Delta \backslash\{0\}$.

If $t \notin \Delta$, then $u_{a}(t) \neq u_{b}(t)$ for any $a \neq b$. The direction of every Stokes ray (8.1) or (8.2) is a continuous functions of $t \notin \Delta$. As $t$ varies in $\mathcal{U}_{\epsilon_{0}}(0) \backslash \Delta$, each one of the rays (8.1) or (8.2) rotates in $\mathcal{R}$.

Remark 9.1. Problems with enumeration of moving Stokes rays. Apparently, we cannot assign a coherent labelling to the rotating rays as $t$ moves in $\mathcal{U}_{\epsilon_{0}}(0) \backslash \Delta$. At a given $t_{0} \in \mathcal{U}_{\epsilon_{0}}(0) \backslash \Delta$, the rays are enumerated according to the choice of an admissible direction $\eta^{\left(t_{0}\right)}$, as in formula (5.7) with $t_{*}=t_{0}$. If $t$ is very close to $t_{0}$, we may choose $\eta^{\left(t_{0}\right)}=\eta^{(t)}$, and we can label the rays in such a way that $\tau_{\sigma}^{(t)}$, $\sigma \in \mathbb{Z}$, is the result of the continuous rotation of $\tau_{\sigma}^{\left(t_{0}\right)}$. Nevertheless, if $t$ moves farther in $\mathcal{U}_{\epsilon_{0}}(0) \backslash \Delta$, then some rays, while rotating, may cross with each other and cross the rays $R\left(\tau^{\left(t_{0}\right)}+k \pi\right), k \in \mathbb{Z}$, which are admissible for $\Lambda\left(t_{0}\right)$. This phenomenon destroys the ordering. Hence, labellings are to be taken independently at $t_{0}$ and at any other $t \in \mathcal{U}_{\epsilon_{0}}(0) \backslash \Delta$, with respect to independent admissible directions $\eta^{\left(t_{0}\right)}$ and $\eta^{(t)}$. In this way, $\tau_{\sigma}^{(t)}$ will not be the deformation of a $\tau_{\sigma}^{\left(t_{0}\right)}$ with the same $\sigma$.

This complication in assigning a coherent numeration to rays and sectors as $t$ varies will be solved in Section 11, by introducing a new labelling, valid for almost all $t \in \mathcal{U}_{\epsilon_{0}}(0)$, induced by the labelling at $t=0$. Before that, we need some topological preparation.

\section{Ray Crossing, Wall Crossing and Cell Decomposition}

We consider an oriented admissible ray $R(\widetilde{\tau})$ for $\Lambda(0)$, with direction $\widetilde{\tau}$, as in Definition 6.2 and we project $\mathcal{R}$ onto $\mathbb{C} \backslash\{0\}$. For $t \in \mathcal{U}_{\epsilon_{0}}(0) \backslash \Delta$, some projected rays associated with $\Lambda(t)$ will be to the left of $l(\widetilde{\tau})$ and some to the right. Moreover, some projected ray may lie exactly on $l(\widetilde{\tau})$, in which case we improperly say that "the ray lies on $l(\widetilde{\tau})$ ". Suppose we start at a value $t_{*} \in \mathcal{U}_{\epsilon_{0}}(0) \backslash \Delta$ such that no rays associated with $\Lambda\left(t_{*}\right)$ lie on $l(\widetilde{\tau})$. If $t$ moves away from $t_{*}$ in $\mathcal{U}_{\epsilon_{0}}(0) \backslash \Delta$, then the directions of Stokes rays change continuously and the projection of two or more rays ${ }^{18}$ may cross $l(\widetilde{\tau})$ as $t$ varies, in which case we say that "two or more rays cross $l(\widetilde{\tau})$ ". Let

$$
\widetilde{\eta}:=\frac{3 \pi}{2}-\widetilde{\tau}
$$

Two or more Stokes rays cross $l(\widetilde{\tau})$ for $t$ belonging to the following crossing locus

$$
X(\widetilde{\tau}):=\bigcup_{1 \leq a<b \leq n}\left\{t \in \mathcal{U}_{\epsilon_{0}}(0) \mid u_{a}(t) \neq u_{b}(t), \quad \arg _{p}\left(u_{a}(t)-u_{b}(t)\right)=\widetilde{\eta} \bmod \pi\right\} .
$$

Let

$$
W(\widetilde{\tau}):=\Delta \cup X(\widetilde{\tau})
$$

Definition 10.1. A $\widetilde{\tau}$-cell is every connected component of the set $\mathcal{U}_{\epsilon_{0}}(0) \backslash W(\widetilde{\tau})$.

$W(\widetilde{\tau})$ is the "wall" of the cells. For $t$ in a $\widetilde{\tau}$-cell, $\Lambda(t)$ is diagonalisable with distinct eigenvalues, and the Stokes rays projected onto $\mathbb{C}$ lie either to the left or to the right of $l(\widetilde{\tau})$. If $t$ varies and hits $W(\widetilde{\tau})$, then either some Stokes rays disappear (when $t \in \Delta$ ), or some rays cross the admissible line $l(\widetilde{\tau}$ ) (when $t \in X(\widetilde{\tau}))$. Notice that

$$
\Delta \cap X(\widetilde{\tau}) \neq \emptyset .
$$

\footnotetext{
${ }^{18}$ Crossing involves always at least two opposite projected rays, which have directions differing by $\pi$. One projection crosses the positive part $l_{+}(\widetilde{\tau})$ of $l(\widetilde{\tau})$, and one projection crosses the negative part $l_{-}(\widetilde{\tau})=l_{+}(\widetilde{\tau} \pm \pi)$.
} 
A cell is open, by definition. If the eigenvalues are linear in $t$, as in (1.15), we will show in Section 10.1 that a cell is simply connected and convex, namely it is a topological cell, so justifying the name. Explicit examples and figures are given in the Appendix.

10.1. Topology of $\widetilde{\tau}$-cells and hyperplane arrangements. In order to study the topology of the $\widetilde{\tau}$-cells, it is convenient to first extend their definition to $\mathbb{C}^{n}$. A $\widetilde{\tau}$-cells in $\mathbb{C}^{n}$ can be proved to be homeomorphic to an open ball, therefore it is a cell in the topological sense. A $\widetilde{\tau}$-cell in $\mathbb{C}^{n}$ is defined to be a connected component of $\mathbb{C}^{n} \backslash\left(\Delta_{\mathbb{C}^{n}} \cup X_{\mathbb{C}^{n}}(\widetilde{\tau})\right)$, where

$$
\begin{aligned}
& \Delta_{\mathbb{C}^{n}}:=\bigcup_{1 \leq a<b \leq n}\left\{u \in \mathbb{C}^{n} \mid u_{a}=u_{b}\right\}, \\
& X_{\mathbb{C}^{n}}(\widetilde{\tau}):=\bigcup_{1 \leq a<b \leq n}\left\{u \in \mathbb{C}^{n} \mid u_{a}-u_{b} \neq 0 \text { and } \arg _{p}\left(u_{a}-u_{b}\right)=\widetilde{\eta} \bmod \pi\right\} .
\end{aligned}
$$

Recall that $\widetilde{\eta}=\frac{3 \pi}{2}-\widetilde{\tau}$.

We identify $\mathbb{C}^{n}$ with $\mathbb{R}^{2 n}$. A point $u=\left(u_{1}, \ldots, u_{n}\right)$ is identified with $(\mathbf{x}, \mathbf{y})=\left(x_{1}, \ldots, x_{n}, y_{1}, \ldots, y_{n}\right)$, by $u_{a}=x_{a}+i y_{a}, 1 \leq a \leq n$. Therefore

a) $\Delta_{\mathbb{C}^{n}}$ is identified with

$$
A:=\bigcup_{1 \leq a<b \leq n}\left\{(\mathbf{x}, \mathbf{y}) \in \mathbb{R}^{2 n} \mid x_{a}-x_{b}=y_{a}-y_{b}=0\right\} .
$$

b) $X_{\mathbb{C}^{n}}(\widetilde{\tau})$ is identified with

$$
B:=\bigcup_{1 \leq a<b \leq n}\left\{(\mathbf{x}, \mathbf{y}) \in \mathbb{R}^{2 n} \mid\left(x_{a}, y_{a}\right) \neq\left(x_{b}, y_{b}\right) \text { and } L_{a b}(\mathbf{x}, \mathbf{y})=0\right\}
$$

where $L_{a b}(\mathbf{x}, \mathbf{y})$ is a linear function

$$
\begin{aligned}
& L_{a b}(\mathbf{x}, \mathbf{y})=\left(y_{a}-y_{b}\right)-\tan \widetilde{\eta}\left(x_{a}-x_{b}\right), \quad \text { for } \widetilde{\eta} \neq \frac{\pi}{2} \bmod \pi, \\
& L_{a b}(\mathbf{x}, \mathbf{y})=x_{a}-x_{b}, \quad \text { for } \widetilde{\eta}=\frac{\pi}{2} \bmod \pi .
\end{aligned}
$$

Hence $A \cup B$ is a union of hyperplanes $H_{a b}$ :

$$
A \cup B=\bigcup_{1 \leq a<b \leq n} H_{a b}, \quad H_{a b}:=\left\{(\mathbf{x}, \mathbf{y}) \in \mathbb{R}^{2 n} \mid L_{a b}(\mathbf{x}, \mathbf{y})=0\right\} .
$$

Note that $L_{a b}(\mathbf{x}, \mathbf{y})=0$ if and only if $L_{b a}(\mathbf{x}, \mathbf{y})=0$, namely $H_{a b}=H_{b a}$. The set $\mathcal{A}=\left\{H_{a b}\right\}_{a<b}$ is known as a hyperplane arrangement in $\mathbb{R}^{2 n}$. We have proved the following lemma

Lemma 10.1. Let $u \in \mathbb{C}^{n}$ be represented as $u=\mathbf{x}+i \mathbf{y},(\mathbf{x}, \mathbf{y}) \in \mathbb{R}^{2 n}$. Then, $\Delta_{\mathbb{C}^{n}} \cup X_{\mathbb{C}^{n}}(\widetilde{\tau})$ is the union of hyperplanes $H_{a b} \in \mathcal{A}$ defined by the linear equations $L_{a b}(\mathbf{x}, \mathbf{y})=0,1 \leq a<b \leq$, as in (10.1), (10.2).

Properties of finite hyperplane arrangements in $\mathbb{R}^{2 n}$ are well knows. In particular, consider the set

$$
\mathbb{R}^{2 n}-\bigcup_{1 \leq a<b \leq n} H_{a b}
$$

A connected component of the above set is called a region of $\mathcal{A}$. It is well known that every region of $\mathcal{A}$ is open and convex, and hence homeomorphic to the interior of an $2 n$-dimensional ball of $\mathbb{R}^{2 n}$. It is therefore $a$ cell in the proper sense. We have proved the following

Proposition 10.1. A $\widetilde{\tau}$-cell in $\mathbb{C}^{n}$ is a cell, namely an open and convex subset of $\mathbb{C}^{n}$, homeomorphic to the open ball $\left\{\left.u \in \mathbb{C}^{n}|| u_{1}\right|^{2}+\cdots+\left|u_{n}\right|^{2}<1\right\}=\left\{(\mathbf{x}, \mathbf{y}) \in \mathbb{R}^{2 n} \mid x_{1}^{2}+\cdots+y_{n}^{2}<1\right\}$.

Remark 10.1. Three hyperplanes with one index in common intersect. Indeed, let $b$ be the common index. Then,

Hence,

$$
\left\{\begin{array}{l}
L_{a b}(\mathbf{x}, \mathbf{y})=0 \\
L_{b c}(\mathbf{x}, \mathbf{y})=0
\end{array} \quad \Longrightarrow \quad L_{a c}(\mathbf{x}, \mathbf{y})=0 .\right.
$$

$$
H_{a b} \cap H_{b c} \subset H_{a c}, \quad H_{b c} \cap H_{a c} \subset H_{a b}, \quad H_{a c} \cap H_{a b} \subset H_{b c} .
$$


Equivalently

$$
H_{a b} \cap H_{b c} \cap H_{a c}=H_{a b} \cap H_{b c}=H_{a b} \cap H_{a c}=H_{b c} \cap H_{a c} .
$$

We now consider $\widetilde{\tau}$-cells in $\mathcal{U}_{\epsilon_{0}}(0)$ in case the eigenvalues of $\Lambda(t)$ are linear in $t$ as in (1.15). The arguments above apply to this case, since $u_{a}=u_{a}(0)+t_{a}$ is a linear translation. Let $u(0)=\left(u_{1}(0), \ldots, u_{n}(0)\right)$ be as in (5.1)-(5.4), so that $u(t)=u(0)+t$. Let us split $u(t)$ into real $(\Re)$ and imaginary $(\Im)$ parts:

$$
u(0)=\mathbf{x}_{0}+i \mathbf{y}_{0}, \quad t=\Re t+i \Im t \quad \Longrightarrow \quad u(t)=\left(\mathbf{x}_{0}+i \mathbf{y}_{0}\right)+(\Re t+i \Im t) .
$$

Here, $\Re t:=\left(\Re t_{1}, \ldots, \Re t_{n}\right) \in \mathbb{R}^{n}$ and $\Im t:=\left(\Im t_{1}, \ldots, \Im t_{n}\right) \in \mathbb{R}^{n}$. Define the hyperplanes

$$
H_{a b}^{\prime}:=\left\{(\Re t, \Im t) \in \mathbb{R}^{n} \mid L_{a b}(\Re t, \Im t)+L_{a b}\left(\mathbf{x}_{0}, \mathbf{y}_{0}\right)=0\right\}, \quad 1 \leq a \neq b \leq n,
$$

and

Then,

$$
\widetilde{H}_{a b}:=H_{a b}^{\prime} \cap \mathcal{U}_{\epsilon_{0}}(0)
$$

$$
\Delta \cup X(\widetilde{\tau})=\bigcup_{1 \leq a<b \leq n} \widetilde{H}_{a b} .
$$

Note that $L_{a b}\left(\mathbf{x}_{0}, \mathbf{y}_{0}\right)=0$ for any $a \neq b$ corresponding to a coalescence $u_{a}(t)-u_{b}(t) \rightarrow 0$ for $t \rightarrow 0$.

Corollary 10.1. If the eigenvalues of $\Lambda(t)$ are linear in $t$ as in (1.15), then a $\widetilde{\tau}$-cell in $\mathcal{U}_{\epsilon_{0}}(0)$ is simply connected.

Proof: Any of the regions of a the hyperplane arrangement with hyperplanes (10.3) is open and convex. $\mathcal{U}_{\epsilon_{0}}(0)$ is a polydisc, hence it is convex. The intersection of a region and $\mathcal{U}_{\epsilon_{0}}(0)$ is then convex and simply connected.

Remark 10.2. The $\widetilde{H}$ 's enjoy the same properties of hyperplanes $H$ 's as in Remark 10.1. In other words, if a Stokes ray associated with the pair $u_{a}(t), u_{b}(t)$ and a Stokes ray associated with $u_{b}(t), u_{c}(t)$ cross an admissible direction $R(\widetilde{\tau} \bmod \pi)$ at some point $t$, then also a ray associated with $u_{a}(t), u_{c}(t)$ does.

Remark 10.3. We anticipate the fact that if $\epsilon_{0}$ is sufficiently small as in Section 14.1 , then $\widetilde{H}_{a b} \cap \mathcal{U}_{\epsilon_{0}}(0)=$ $\emptyset$ for any $a \neq b$ such that for $t \rightarrow 0, u_{a}(t) \rightarrow \lambda_{i}$ and $u_{b}(t) \rightarrow \lambda_{j}$ with $1 \leq i \neq j \leq s\left(\right.$ i.e. $\left.u_{a}(0) \neq u_{b}(0)\right)$. See below Remark 14.1 for explanations.

\section{SECTORS $\mathcal{S}_{\nu}(t)$ AND $\mathcal{S}_{\nu}(K)$}

We introduce $t$-dependent sectors, which serve to define Stokes matrices of $Y(z, t)$ of Corollary 5.1 in a consistent way w.r.t. matrices of $\stackrel{\circ}{Y}(z)$ of Theorem 4.1 .

Definition 11.1 (Sectors $\left.\mathcal{S}_{\nu+k \mu}(t)\right)$. Let $\tau_{\nu}<\widetilde{\tau}<\tau_{\nu+1}$, and $k \in \mathbb{Z}$. Let $t \in \mathcal{U}_{\epsilon_{0}}(0) \backslash X(\widetilde{\tau})$. We define $\mathcal{S}_{\nu+k \mu}(t)$ to be the sector containing the closed sector $\bar{S}(\widetilde{\tau}-\pi+k \pi, \widetilde{\tau}+k \pi)$, and extending up to the nearest Stokes rays of $\Lambda(t)$ outside $\bar{S}(\widetilde{\tau}-\pi+k \pi, \widetilde{\tau}+k \pi)$.

The definition implies that

$$
\mathcal{S}_{\nu+k \mu}(t) \subset \mathcal{S}_{\nu+k \mu}, \quad \mathcal{S}_{\nu+k \mu}(0)=\mathcal{S}_{\nu+k \mu}
$$

For simplicity, put $k=0$. Note that $\mathcal{S}_{\nu}(t)$ is uniquely defined and contains the set of basic Stokes rays of $\Lambda(t)$ lying in $S(\widetilde{\tau}-\pi, \widetilde{\tau})$. We point out the following facts:

- Due to the continuous dependence on $t$ of the directions of Stokes rays for $t \notin \Delta$, then $\mathcal{S}_{\nu}(t)$ continuously deforms as $t$ varies in a $\widetilde{\tau}$ cell.

- $\mathcal{S}_{\nu}(t)$ is "discontinuous" at $\Delta$, by which we mean that some Stokes rays disappear at points of $\Delta$.

- $\mathcal{S}_{\nu}(t)$ is "discontinuous" at $X(\widetilde{\tau})$, because one or more Stokes rays cross the admissible ray $R(\widetilde{\tau})$ (this is why $\mathcal{S}_{\nu}(t)$ has not been defined at $X(\widetilde{\tau})$ ). More precisely, consider a continuous monotone curve $t=t(x), x$ belonging to a real interval, which for one pair $(a, b)$ intersects $\widetilde{H}_{a b} \backslash \Delta$ at $x=x_{*}$ (recall that $\widetilde{H}_{a b}$ is define in (10.4)). Hence, the curve passes from one cell to another cell, which are separated by $\widetilde{H}_{a b}$. A Stokes ray associated with $\left(u_{a}(t), u_{b}(t)\right)$ crosses $R(\widetilde{\tau})$ when $t=t\left(x_{*}\right)$. Then $\mathcal{S}_{\nu}(t(x))$ has a discontinuous jump at $x_{*}$. 

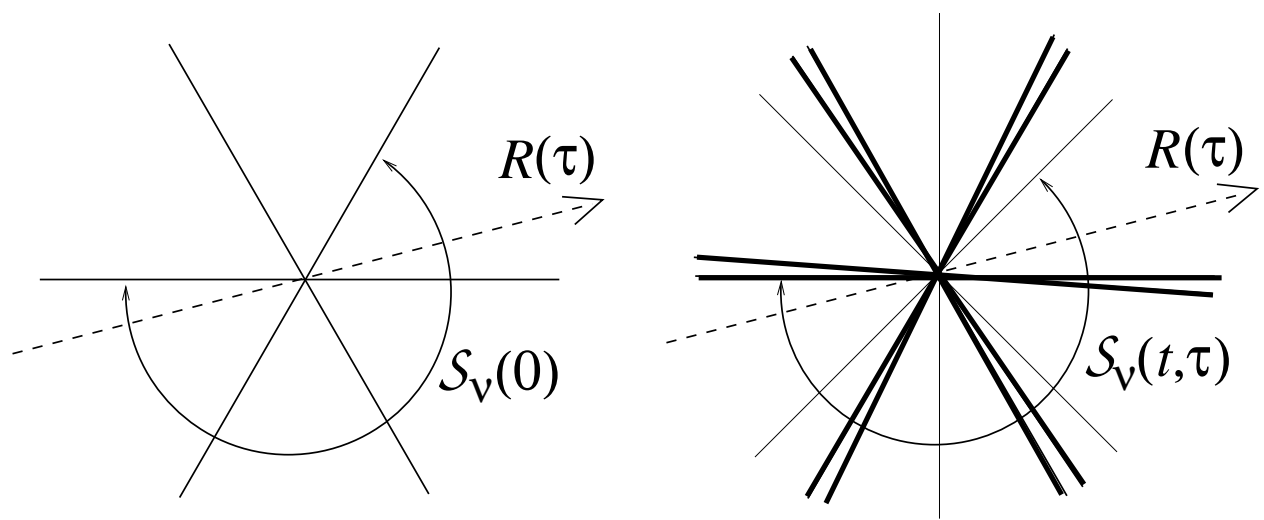

FiguRE 2. In the left figure $t=0$ and the sector $\mathcal{S}_{\nu} \equiv \mathcal{S}_{\nu}(0)$ is represented in a sheet of the universal covering $\mathcal{R}$. The dashed line represents $R(\widetilde{\tau}) \cup R(\widetilde{\tau}-\pi)$. The arrow is that of the oriented ray $R(\widetilde{\tau})$. The rays are the Stokes rays associated with couples $\lambda_{i}, \lambda_{j}, 1 \leq i \neq j \leq s$. In the right figure $t$ slightly differs from $t=0$; the rays in bold are small deformations of the rays appearing in the left figure, associated with couples $u_{a}(t), u_{b}(t)$ s.t. $u_{a}(0)=\lambda_{i}, u_{b}(0)=\lambda_{j}$ with $i \neq j$. The rays in finer tone are the rays associated with couples such that $u_{a}(0)=u_{b}(0)=\lambda_{i}$. The sector $\mathcal{S}_{\nu}(t)=\mathcal{S}_{\nu}(t, \widetilde{\tau})$ is represented.

The above observations assure that the following definition is well posed.

Definition 11.2 (Sector $\left.\mathcal{S}_{\nu}(K)\right)$. Let $K$ be a compact subset of a $\widetilde{\tau}$-cell. We define

$$
\mathcal{S}_{\nu}(K):=\bigcap_{t \in K} \mathcal{S}_{\nu}(t) \subset \mathcal{S}_{\nu}
$$

By the definitions, $\mathcal{S}_{\nu}(t)$ and $\mathcal{S}_{\nu}(K)$ have the angular width strictly greater than $\pi$ and they contain the admissible ray $R(\widetilde{\tau})$ of Definition 6.2. Moreover $\mathcal{S}_{\nu}\left(K_{1}\right) \supset \mathcal{S}_{\nu}\left(K_{2}\right)$ for $K_{1} \subset K_{2}$, and $\mathcal{S}_{\nu}\left(K_{1} \cup K_{2}\right)=$ $\mathcal{S}_{\nu}\left(K_{1}\right) \cap \mathcal{S}_{\nu}\left(K_{2}\right)$. Below in the paper we will consider a simply connected subset $\mathcal{V}$ of a $\widetilde{\tau}$-cell, such that the closure $\overline{\mathcal{V}}$ is also contained in the cell, and take

$$
K=\overline{\mathcal{V}}
$$

Remark 11.1. A more precise notation could be used as follows:

$$
\mathcal{S}_{\nu}(t)=\mathcal{S}_{\nu}(t ; \tilde{\tau}),
$$

to keep track of $\widetilde{\tau}$, because for given $\nu$ and two different choices of $\widetilde{\tau} \in\left(\tau_{\nu}, \tau_{\nu+1}\right)$, then the resulting $\mathcal{S}_{\nu}(t)$ 's may be different. Figures 2 and 3 show two different $\mathcal{S}_{\nu}(t)$, according to two choices of $\widetilde{\tau}$. As a consequence, while in Definition 11.1 we could well define $\mathcal{S}_{\nu+k \mu}(t) \subset \mathcal{S}_{\nu+k \mu}$, for any $k \in \mathbb{Z}$, we cannot define sectors $\mathcal{S}_{\nu+1}(t), \mathcal{S}_{\nu+2}(t), \ldots, \mathcal{S}_{\nu+\mu-1}(t)$.

\section{Fundamental Solutions $Y_{\nu}(z, t)$ and $\operatorname{Stokes~Matrices~} \mathbb{S}_{\nu}(t)$}

Let $\tau_{\nu}<\widetilde{\tau}<\tau_{\nu+1}$. We show that, if $t_{0} \notin \Delta$ belongs to a $\widetilde{\tau}$-cell, we can extend the asymptotic behaviour (5.10) of Corollary 5.1 from $\bar{S}^{\left(t_{0}\right)}(\alpha, \beta)$ to $\mathcal{S}_{\nu}(t)$. The fundamental matrix of Corollary 5.1 will then be denoted by $Y_{\nu}(z, t)$.

Proposition 12.1 (Solution $Y_{\nu}(z, t)$ with asymptotics on $\left.\mathcal{S}_{\nu}(t), t \in \mathcal{U}_{\rho}\left(t_{0}\right)\right)$. Let Assumption 1 hold for the system (2.1). Let $t_{0}$ belong to a $\widetilde{\tau}$-cell. For any $\nu \in \mathbb{Z}$ there exists $\mathcal{U}_{\rho}\left(t_{0}\right)$ contained in the cell of $t_{0}$ and a unique fundamental solution of the system (2.1) as in Corollary 5.1 of the form

$$
Y_{\nu}(z, t)=G_{0}(t) \mathcal{G}_{\nu}(z, t) z^{B_{1}(t)} e^{\Lambda(t) z},
$$



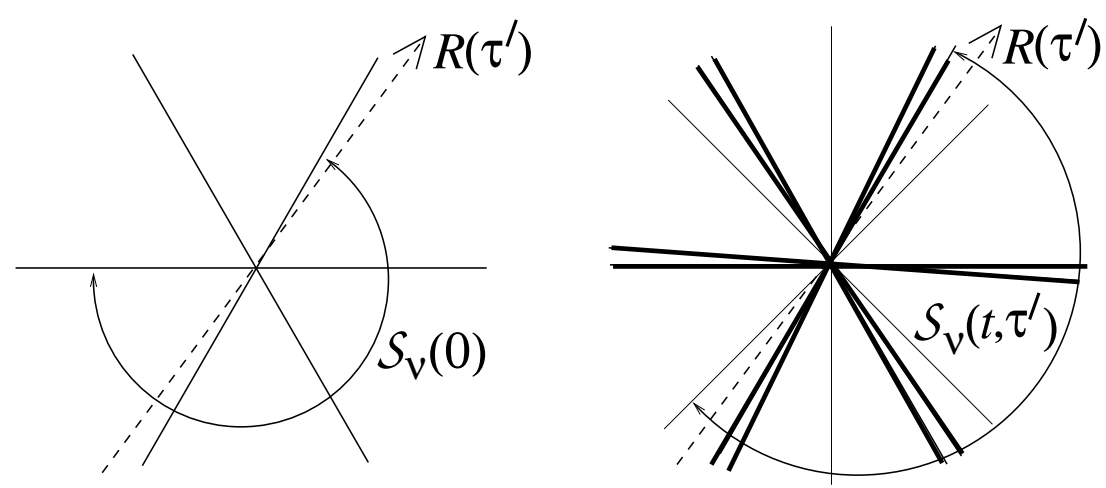

Figure 3. The explanation for this figure is the same as for Figure 2, but $\widetilde{\tau}^{\prime} \neq \widetilde{\tau}$. $\mathcal{S}_{\nu} \equiv \mathcal{S}_{\nu}(0)$ is the same, but $\mathcal{S}_{\nu}(t)=\mathcal{S}_{\nu}\left(t, \widetilde{\tau}^{\prime}\right)$ differs from $\mathcal{S}_{\nu}(t, \widetilde{\tau})$ of figure 2.

holomorphic in $(z, t) \in\{z \in \mathcal{R}|| z \mid \geq N\} \times \mathcal{U}_{\rho}\left(t_{0}\right)$, with asymptotic behaviour (5.10) extended to $\mathcal{S}_{\nu}(t)$, $t \in \mathcal{U}_{\rho}\left(t_{0}\right)$. Namely $\forall t \in \mathcal{U}_{\rho}\left(t_{0}\right)$ the following asymptotic expansion holds:

$$
\mathcal{G}_{\nu}(z, t) \sim I+\sum_{k=1}^{\infty} F_{k}(t) z^{-k}, \quad z \rightarrow \infty, \quad z \in \mathcal{S}_{\nu}(t) .
$$

The asymptotics (12.2) restricted to $z \in \mathcal{S}_{\nu}\left(\mathcal{U}_{\rho}\left(t_{0}\right)\right)$ is uniform in the compact polydisc $\mathcal{U}_{\rho}\left(t_{0}\right)$.

Note: Recall that by definition of asymptotics, the last sentence of the above Proposition means that the asymptotics (12.2) is uniform in the compact polydisc $\mathcal{U}_{\rho}\left(t_{0}\right)$ when $z \rightarrow \infty$ in any proper closed subsector of $\mathcal{S}_{\nu}\left(\mathcal{U}_{\rho}\left(t_{0}\right)\right)$.

Proof: In Theorem 5.1 choose $\bar{S}^{\left(t_{0}\right)}(\alpha, \beta)=\bar{S}(\widetilde{\tau}-\pi, \widetilde{\tau})$. This contains a set of basic Stokes rays of $\Lambda\left(t_{0}\right)$ and of $\Lambda(t)$ for any $t$ in the cell of $t_{0}$. Then, Sibuya's Theorem 5.1 and Corollary 5.1 apply, with fundamental solution $Y(z, t)$ defined for $t$ in some $\mathcal{U}_{\rho}\left(t_{0}\right)$. It is always possible to restrict $\rho$ so that $\mathcal{U}_{\rho}\left(t_{0}\right)$ is all contained in the cell.

- [Extension to $\left.\mathcal{S}_{\nu}(t)\right]$ For $t \in \mathcal{U}_{\rho}\left(t_{0}\right)$, the sector containing $S(\widetilde{\tau}-\pi, \widetilde{\tau})$ and extending up to the nearest Stokes rays outside is $\mathcal{S}_{\nu}(t)$, by definition. Hence there exists a labelling as in Section 5.1, and a $\sigma \in \mathbb{Z}$, such that $\mathcal{S}_{\nu}\left(t_{0}\right)=\mathcal{S}_{\sigma}^{\left(t_{0}\right)}$. The Extension Theorem and the Uniqueness Theorem can be applied to $Y(z, t)$ for any fixed $t$, because $S(\widetilde{\tau}-\pi, \widetilde{\tau})$ contains a set of basic Stokes rays. Hence, for any $t \in \mathcal{U}_{\rho}\left(t_{0}\right)$ the solution $Y(z, t)$ is unique with the asymptotic behaviour (5.10) for $z \rightarrow \infty$ in $\mathcal{S}_{\nu}(t)$.

- [Uniformity in $\mathcal{S}_{\nu}\left(\mathcal{U}_{\rho}\left(t_{0}\right)\right)$ ] Clearly, $\mathcal{S}_{\nu}\left(\mathcal{U}_{\rho}\left(t_{0}\right)\right) \supset \bar{S}(\widetilde{\tau}-\pi, \widetilde{\tau})$. Since $\mathcal{S}_{\nu}\left(\mathcal{U}_{\rho}\left(t_{0}\right)\right) \subset \mathcal{S}_{\nu}(t)$ for any $t \in \mathcal{U}_{\rho}\left(t_{0}\right)$, the asymptotics (12.2) holds also in $\mathcal{S}_{\nu}\left(\mathcal{U}_{\rho}\left(t_{0}\right)\right)$. Moreover, the asymptotics is uniform in $\mathcal{U}_{\rho}\left(t_{0}\right)$ if $z \rightarrow \infty$ in $\bar{S}(\widetilde{\tau}-\pi, \widetilde{\tau})$, by Theorem 5.1 and Corollary 5.1. We apply the same proof of the Extension Lemma 6.2 as follows. Let $\theta_{L}$ and $\theta_{R}$ be the directions of the left and right boundary rays of $\mathcal{S}_{\nu}\left(\mathcal{U}_{\rho}\left(t_{0}\right)\right)$ (i.e. $\overline{\mathcal{S}}_{\nu}\left(\mathcal{U}_{\rho}\left(t_{0}\right)\right)=\bar{S}\left(\theta_{R}, \theta_{L}\right)$ ). Let $\bar{S}_{1}:=\bar{S}(\phi, \psi)$, for $\theta_{R}+\pi<\phi<\psi<\theta_{L}$, and $\bar{S}_{2}:=\bar{S}\left(\phi^{\prime}, \psi^{\prime}\right)$ for $\theta_{R}<\phi^{\prime}<\psi^{\prime}<\theta_{L}-\pi$. Let us consider $\bar{S}_{1}$. By construction, $\bar{S}_{1}$ does not contain Stokes rays of $\Lambda(t)$ for any $t \in \mathcal{U}_{\rho}\left(t_{0}\right)$, and so, by Theorem 5.1 now applied with a $\bar{S}^{\left(t_{0}\right)}=\bar{S}_{1}$, there exists $\tilde{Y}(z, t) \sim Y_{F}(z, t)$, for $z \rightarrow \infty$ in $\bar{S}_{1}$, uniformly in $\left|t-t_{0}\right| \leq \rho_{1}$, for suitable $\rho_{1}>0$. Moreover, $Y(z, t)=\widetilde{Y}(z, t) C(t)$, where $C(t)$ is an invertible holomorphic matrix in $\left|t-t_{0}\right| \leq \min \left(\rho, \rho_{1}\right)$. The matrix entries satisfy $e^{\left(u_{a}(t)-u_{b}(t)\right) z} C_{a b}(t)=\widetilde{\mathcal{G}}(z, t)^{-1} \mathcal{G}(z, t) \sim \delta_{a b}, a, b=1, \ldots, n$, for $\left|t-t_{0}\right| \leq \min \left(\rho, \rho_{1}\right)$ and $z \rightarrow \infty, z \in \bar{S}(\widetilde{\tau}-\pi, \widetilde{\tau}) \cap \bar{S}_{1}$. Since $\Re\left(\left(u_{a}(t)-u_{b}(t)\right) z\right)$ does not change sign for $t$ in the cell and $z \in \bar{S}_{1}$, then $Y(z, t) \sim Y_{F}(z, t)$ also for $z \in \bar{S}(\widetilde{\tau}-\pi, \widetilde{\tau}) \cup \bar{S}_{1}$, uniformly in $\left|t-t_{0}\right| \leq \min \left(\rho, \rho_{1}\right)$. The same arguments for $\bar{S}_{2}$ allow to conclude that $Y(z, t) \sim Y_{F}(z, t)$ for $z \in \bar{S}(\widetilde{\tau}-\pi, \widetilde{\tau}) \cup \bar{S}_{1} \cup \bar{S}_{2}$, uniformly in $\left|t-t_{0}\right| \leq \min \left(\rho, \rho_{1}, \rho_{2}\right)$. Finally, from the proof given by Sibuya of Theorem 5.1 (cf. [66], especially from page 44 on) it follows that $\rho_{1}$ and $\rho_{2}$ are greater or equal to $\rho$. The proof is concluded. We denote $Y(z, t)$ with $Y_{\nu}(z, t)$. 
Definition 12.1 (Stokes matrices $\mathbb{S}_{\nu+k \mu}(t)$ ). The Stokes matrix $\mathbb{S}_{\nu+k \mu}(t), k \in \mathbb{Z}$, is defined for $t \in \mathcal{U}_{\rho}\left(t_{0}\right)$ of Proposition 12.1 by,

$$
Y_{\nu+(k+1) \mu}(z, t)=Y_{\nu+k \mu}(z, t) \mathbb{S}_{\nu+k \mu}(t), \quad z \in \mathcal{R},
$$

where the $Y_{\nu+k \mu}(z, t)$ and $Y_{\nu+(k+1) \mu}(z, t)$ are as in Proposition 12.1.

$$
\mathbb{S}_{\nu+k \mu}(t) \text { is holomorphic in } t \in \mathcal{U}_{\rho}\left(t_{0}\right) \text {, because so are } Y_{\nu+(k+1) \mu}(z, t) \text { and } Y_{\nu+k \mu}(z, t) \text {. }
$$

\section{Analytic Continuation of $Y_{\nu}(z, t)$ on a Cell preserving the Asymptotics}

Proposition 13.1 (Continuation of $Y_{\nu}(z, t)$ preserving the asymptotics, along a curve in a cell). Let Assumption 1 hold for the system (2.1). The fundamental solution $Y_{\nu}(z, t)$ of Proposition 12.1 holomorphic in $t \in \mathcal{U}_{\rho}\left(t_{0}\right)$ admits t-analytic continuation along any curve contained in the $\widetilde{\tau}$-cell of $t_{0}$, and maintains its asymptotics (12.2) for $z \rightarrow \infty, z \in \mathcal{S}_{\nu}(t)$, for any $t$ belonging to a neighbourhood of the curve. The asymptotics is uniform in a closed tubular neighbourhood $U$ of the curve for $z \rightarrow \infty$ in (any proper subsector of) $\mathcal{S}_{\nu}(U)$.

Proof: Let $Y_{\nu}(z, t), t \in \mathcal{U}_{\rho}\left(t_{0}\right)$ be as in Proposition 12.1. Join $t_{0}$ to a point $t_{\text {final }}$, belonging to the $\widetilde{\tau}$-cell of $t_{0}$ and not belonging to $\mathcal{U}_{\rho}\left(t_{0}\right)$, by a curve whose support is contained in the $\widetilde{\tau}$-cell. Let $t_{1} \in \partial \mathcal{U}_{\rho}\left(t_{0}\right)$ be the intersection point with the curve. Theorem 5.1 and its Corollary 5.1 can be applied at $t_{1}$, with sector $\mathcal{S}_{\sigma}^{\left(t_{1}\right)} \equiv \mathcal{S}_{\nu}\left(t_{1}\right)$, by definition. By Proposition 12.1, there exists a unique fundamental solution, which we temporarily denote $Y_{\nu}^{(1)}(z, t)$, with asymptotics $(12.2)$ for $z \rightarrow \infty, z \in \mathcal{S}_{\nu}(t), t \in \mathcal{U}_{\rho_{1}}\left(t_{1}\right)$. Here $\rho_{1}$ is possibly restricted so that $\mathcal{U}_{\rho_{1}}\left(t_{1}\right)$ is contained in the cell. The asymptotics is uniform in $\mathcal{U}_{\rho_{1}}\left(t_{1}\right)$ for $z \rightarrow \infty$ in $\mathcal{S}_{\nu}\left(\mathcal{U}_{\rho_{1}}\left(t_{1}\right)\right)$. Now, when $t \in \mathcal{U}_{\rho}\left(t_{0}\right) \cap \mathcal{U}_{\rho_{1}}\left(t_{1}\right)$, both $Y_{\nu}(z, t)$ and $Y_{\nu}^{(1)}(z, t)$ are defined, with the same asymptotic behaviour (12.2) for $z \rightarrow \infty, z \in \mathcal{S}_{\nu}\left(\mathcal{U}_{\rho}\left(t_{0}\right)\right) \cap \mathcal{S}_{\nu}\left(\mathcal{U}_{\rho_{1}}\left(t_{1}\right)\right)$, uniform in $t \in \mathcal{U}_{\rho}\left(t_{0}\right) \cap \mathcal{U}_{\rho_{1}}\left(t_{1}\right)$. Moreover, $\mathcal{S}_{\nu}\left(\mathcal{U}_{\rho}\left(t_{0}\right)\right) \cap \mathcal{S}_{\nu}\left(\mathcal{U}_{\rho_{1}}\left(t_{1}\right)\right)$ has central opening angle strictly greater than $\pi$ because both $\mathcal{U}_{\rho}\left(t_{0}\right)$ and $\mathcal{U}_{\rho_{1}}\left(t_{1}\right)$ are contained in the cell. By uniqueness it follows that $Y_{\nu}(z, t)=Y_{\nu}^{(1)}(z, t)$ for $t \in \mathcal{U}_{\rho}\left(t_{0}\right) \cap \mathcal{U}_{\rho_{1}}\left(t_{1}\right)$. This gives the $t$-analytic continuation of $Y_{\nu}(z, t)$ on $\mathcal{U}_{\rho}\left(t_{0}\right) \cup \mathcal{U}_{\rho_{1}}\left(t_{1}\right)$. The procedure can be repeated for a sequence of neighbourhoods $\mathcal{U}_{\rho_{n}}\left(t_{n}\right), n=1,2,3, \ldots$ $\left(t_{n}\right.$ is point of intersection of the curve with $\left.\mathcal{U}_{\rho_{n-1}}\left(t_{n-1}\right)\right)$. Consider $U:=\bigcup_{n} \mathcal{U}_{\rho_{n}}\left(t_{n}\right)$. If $t_{\text {final }}$ is an internal point of $\in U$, the proof is completed and $\mathcal{U}_{\rho_{n}}\left(t_{n}\right)$ is a finite sequence. If not, the point $t_{*}$ of intersection of $\partial U$ with the curve either precedes $t_{\text {final }}$, or $t_{*}=t_{\text {final }} \in \partial U$. Since $t_{*}$ belongs to the cell, Proposition 12.1 can be applied. The sector $\mathcal{S}_{\sigma_{*}}^{\left(t_{*}\right)}, \sigma_{*} \in \mathbb{Z}$, prescribed by Theorem 5.1 and Corollary 5.1 coincides with $\mathcal{S}_{\nu}\left(t_{*}\right)$, by definition. Therefore, the analytic continuation is feasible in a $\mathcal{U}_{\rho^{*}}\left(t_{*}\right)$, as in the construction above. We can add $\mathcal{U}_{\rho^{*}}\left(t_{*}\right)$ to $U$. In this way, $t_{\text {final }}$ is always reached by a finite sequence, and $U$ is compact. By construction, the asymptotics is uniform in any compact subset $K \subset U$, including also $K \equiv U$, for $z \rightarrow \infty, z \in \mathcal{S}_{\nu}(K)$.

Corollary 13.1. (Analytic continuation of $Y_{\nu}(z, t)$ preserving the asymptotics on the whole cell - case of eigenvalues (1.15)). Let Assumption 1 hold for the system (2.1). If the eigenvalues of $\Lambda(t)$ are linear in $t$ as in (1.15) then $Y_{\nu}(z, t)$ of Proposition 12.1 is holomorphic on the whole $\widetilde{\tau}$-cell, with asymptotics (12.2) for $z \rightarrow \infty$ in $\mathcal{S}_{\nu}(t)$, for any $t$ in the cell. For any compact subset $K$ of the cell, the asymptotics (12.2) for $z \rightarrow \infty, z \in \mathcal{S}_{\nu}(K)$, is uniform in $t \in K$.

Proof: If the eigenvalues of $\Lambda(t)$ are linear in $t$ as in (1.15), then any $\widetilde{\tau}$-cell is simply connected (see Corollary 10.1). Hence, the continuation of $Y_{\nu}(z, t)$ is independent of the curve.

- Notation: If $c$ is the $\widetilde{\tau}$-cell of Corollary 13.1, the following notation will be used

$$
Y_{\nu}(z, t)=Y_{\nu}(z, t ; \widetilde{\tau}, c), \quad t \in c .
$$




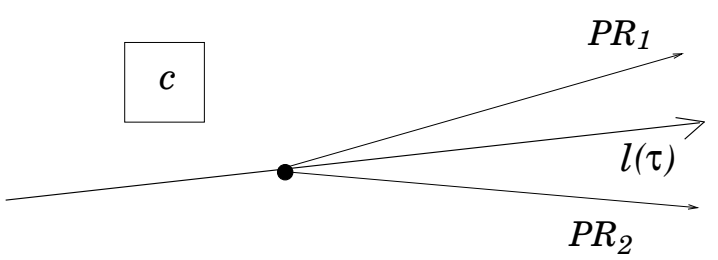

FiguRE 4. Configuration of rays corresponding to the cell $c$ of figures 11 and 12 .

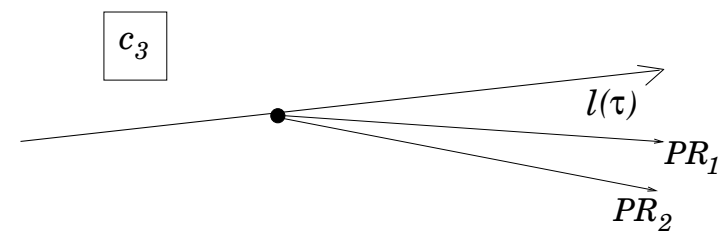

Figure 6. Configuration of rays corresponding to the cell $c_{3}$ of figures 11 and 12 .

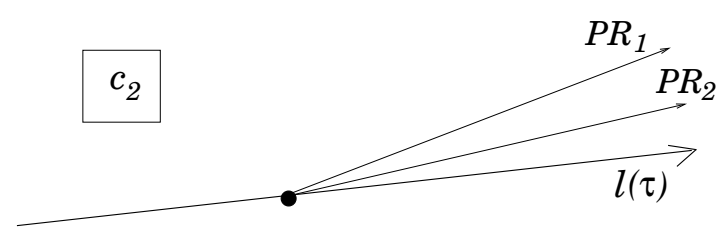

Figure 5. Configuration of rays corresponding to the cell $c_{2}$ of figures 11 and 12 .

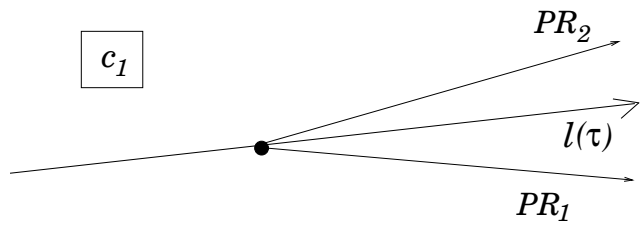

Figure 7. Configuration of rays corresponding to the cell $c_{1}$ of figures 11 and 12 .

13.1. Analytic continuation of $Y_{\nu}(z, t ; \widetilde{\tau}, c)$ preserving the asymptotics beyond $\partial c$. Let the eigenvalues of $\Lambda(t)$ be linear in $t$ as in (1.15). The analytic continuation of Corollary 13.1 and the asymptotics (12.2) can be extended to values of $t$ a little bit outside the cell. This is achieved by a small variation $\widetilde{\tau} \mapsto \widetilde{\tau} \pm \varepsilon$, for $\varepsilon>0$ sufficiently small.

Recall that the Stokes rays in $\mathcal{R}$ associated with the pair $\left(u_{a}(t), u_{b}(t)\right)$ and $\left(u_{b}(t), u_{a}(t)\right), a \neq b$, have respectively directions

$\arg z=\frac{3 \pi}{2}-\arg _{p}\left(u_{a}(t)-u_{b}(t)\right)+2 N \pi \quad$ and $\quad \arg z=\frac{3 \pi}{2}-\arg _{p}\left(u_{b}(t)-u_{a}(t)\right)+2 N \pi, \quad N \in \mathbb{Z}$.

Thus, their projections onto $\mathbb{C}$ are the following opposite rays

$$
P R_{a b}(t):=\left\{z \in \mathbb{C} \mid z=-i \rho\left(\bar{u}_{a}(t)-\bar{u}_{b}(t)\right)\right\}, \quad P R_{b a}(t):=\left\{z \in \mathbb{C} \mid z=-i \rho\left(\bar{u}_{b}(t)-\bar{u}_{a}(t)\right)\right\} .
$$

For $t \notin W(\widetilde{\tau})$, a ray $P R_{a b}(t)$ lies either in the half plane to the left or to the right of the oriented admissible line $l(\widetilde{\tau})$. For $t \notin W(\widetilde{\tau})$, the finite set of projected rays is the union of the two disjoint subsets of (projected) rays to the left and to the right of $l(\widetilde{\tau})$ respectively. Now, for $t$ varying inside a cell $c$, the projected rays never cross $l(\widetilde{\tau})$. On the other hand, if $t$ and $t^{\prime}$ belong to different cells $c$ and $c^{\prime}$, then the two subsets of rays to the right and the left of $l(\widetilde{\tau})$ which are associated with $t$ do not coincide with the two subsets associated with $t^{\prime}$. These simple considerations imply the following:

Proposition 13.2. A $\widetilde{\tau}$-cell is uniquely characterised by the subset of projected rays which lie to the left of $l(\widetilde{\tau})$.

Definition 13.1. A point $t_{*} \in \widetilde{H}_{a b} \backslash \Delta$ is simple if $t_{*} \notin \widetilde{H}_{a b} \cap \widetilde{H}_{a^{\prime} b^{\prime}}$ for any $\left(a^{\prime}, b^{\prime}\right) \neq(a, b)$.

If $t$ varies along a curve crossing the boundary $\partial c$ of a cell $c$ at a simple point belonging to $\widetilde{H}_{a b} \backslash \Delta$, for some $a \neq b$, the ray $P R_{a b}(t)$ crosses either $l_{+}(\widetilde{\tau})$ or $l_{-}(\widetilde{\tau})$, while $P R_{b a}(t)$ crosses either $l_{-}(\widetilde{\tau})$ or $l_{+}(\widetilde{\tau})$. Since only $P R_{a b}(t)$ and $P R_{b a}(t)$ have crossed $l(\widetilde{\tau})$, then by Proposition 13.2 there is only one neighbouring cell $c^{\prime}$ sharing the boundary $\widetilde{H}_{a b}$ with $c$. On the other hand, if the curve crosses $\partial c \backslash \Delta$ at a non simple point, then two or more rays simultaneously cross $l_{+}(\widetilde{\tau})$ (and the opposite ones cross $l_{-}(\widetilde{\tau})$ ). For example, if the crossing occurs at $\left(\widetilde{H}_{a b} \cap \widetilde{H}_{a^{\prime} b^{\prime}}\right) \backslash \Delta$ then there are three cells, call them $c_{1}, c_{2}, c_{3}$, sharing common boundary $\left(\widetilde{H}_{a b} \cap \widetilde{H}_{a^{\prime} b^{\prime}}\right) \backslash \Delta$ with $c$. Looking at the configuration of Stokes rays as in the figures $4,5,6,7$, we conclude that out of the three cells $c_{1}, c_{2}, c_{3}$, there is one, say it is $c_{1}$, such that the transition from $c$ to $c_{1}$ occurs with a double crossing of Stokes rays (figure 7), namely at a non-simple 


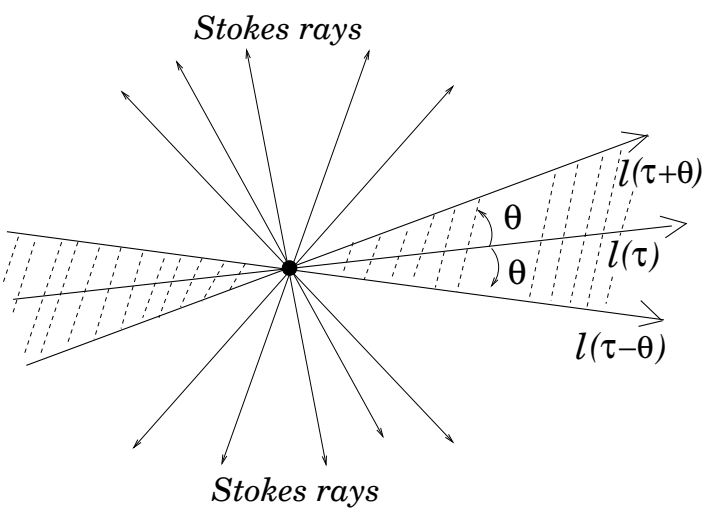

Figure 8. The two closed sectors of amplitude $2 \vartheta$, not containing Stokes rays when $t \in \bar{U}$.

point; while for the remaining $c_{2}$ and $c_{3}$ the transition occurs at simple points. In figures $4,5,6,7, P R_{1}$ stands for $P R_{a b}(t)$ (or $P R_{b a}(t)$ ) and $P R_{2}$ stands for $P R_{a^{\prime} b^{\prime}}(t)$ (or $P R_{b^{\prime} a^{\prime}}(t)$ ). The transition between figure 4 and 7 is between $c$ and $c_{1}$ of figure 11, through non simple points of $\left(\widetilde{H}_{a b} \cap \widetilde{H}_{a^{\prime} b^{\prime}}\right) \backslash \Delta$.

Remark 13.1. Recall that for any $a \neq b, \widetilde{H}_{a b} \cap \Delta \neq \emptyset$. Therefore, when we discuss analytic continuation, this requires crossing of "hyperplanes" $\widetilde{H}_{a b} \backslash \Delta$.

Proposition 13.3 (Continuation slightly beyond the cell, preserving asymptotics). Let the assumptions of Corollary 13.1 hold. Let $c$ and $c^{\prime}$ be $\widetilde{\tau}$-cells such that $\partial c \cap \partial c^{\prime} \neq \emptyset$. If $\partial c \cap \partial c^{\prime}$ does not coincide with the multiple intersection of two or more $\widetilde{H}_{a b}$ 's, then $Y_{\nu}(z, t ; \widetilde{\tau}, c)$ has analytic continuation, with asymptotics (12.2) in $\mathcal{S}_{\nu}(t)$, for $t$ slightly beyond $\partial c \backslash \Delta$ into $c^{\prime}$. The asymptotics for $z \rightarrow \infty$ in $\mathcal{S}_{\nu}(K)$ is uniform in any compact subset $K$ of the extended cell. Equivalently, $Y_{\nu}(z, t ; \widetilde{\tau}, c)$ can be analytically continued along any curve crossing $\partial c \backslash \Delta$ at a simple point and ending slightly beyond $\partial c \backslash \Delta$ in the neighbouring cell $c^{\prime}$.

Proof: Let $U$ be an open connected subset of the $\widetilde{\tau}$-cell $c$, such that $\bar{U}$ is contained in $c$. There exists a small $\vartheta=\vartheta(U)>0$ such that for any $t \in \bar{U}$ the projected Stokes rays of $\Lambda(t)$ lie outside the two closed sectors containing $l(\widetilde{\tau})$ and bounded by $l(\widetilde{\tau}+\theta)$ and $l(\widetilde{\tau}-\theta)$, as in figure 8 . Let $\varepsilon \in[0, \vartheta]$. All lines $l(\widetilde{\tau} \pm \varepsilon)$ are admissible for the Stokes rays, when $t \in \bar{U}$. Consider the subset of projected Stokes rays to the left of $l(\widetilde{\tau})$. It uniquely identifies (cf. Proposition 13.2) the $(\widetilde{\tau}+\varepsilon)$-cell and the $(\widetilde{\tau}-\varepsilon)$-cell obtained by deforming the boundaries of $c$ when $\widetilde{\tau} \mapsto \widetilde{\tau}+\varepsilon$ and $\widetilde{\tau} \mapsto \widetilde{\tau}-\varepsilon$ respectively (recall that $L_{a b}$ in (10.3) depends on $\widetilde{\eta}=3 \pi / 2-\widetilde{\tau}$ ). Call these cells $c_{\varepsilon}$ and $c_{-\varepsilon}$. By construction

$$
\begin{aligned}
& \bar{U} \subset c \cap c_{ \pm \varepsilon}, \quad \varepsilon \in[0, \vartheta], \\
& Y_{\nu}(z, t ; \widetilde{\tau}, c)=Y_{\nu}\left(z, t ; \widetilde{\tau} \pm \varepsilon, c_{ \pm \varepsilon}\right), \quad t \in U .
\end{aligned}
$$

The last equality follows from the definition of $Y_{\nu}$, its uniqueness and Corollary 13.1. Indeed, the analytic continuation explained in the proof of Proposition 13.1 can be repeated for the function $Y_{\nu}\left(z, t ; \widetilde{\tau} \pm \varepsilon, c_{ \pm \varepsilon}\right)$ initially defined in a neighbourhood of $t_{0}$ contained in $\bar{U}$, but with cell partition determined by $\widetilde{\tau} \pm \varepsilon$. Moreover, by uniqueness of solutions with asymptotics, it follows that $Y_{\nu}(z, t ; \widetilde{\tau}, c)=Y_{\nu}\left(z, t ; \widetilde{\tau} \pm \varepsilon, c_{ \pm \varepsilon}\right)$ for $t \in U$. Therefore, $Y_{\nu}(z, t ; \widetilde{\tau}, c)$ has analytic continuation to $c_{ \pm \varepsilon}$. Now,

$$
c_{ \pm \varepsilon} \cap\{\text { union of cells sharing boundary with } c\} \neq \emptyset .
$$

Then, the analytic continuation of $Y_{\nu}(z, t ; \widetilde{\tau}, c)$ obtained above is actually defined in a $t$-domain bigger than $c$. We characterise this domain, showing that it intersect any cell $c^{\prime}$ which is a neighbour of $c$, and such that $\partial c \cap \partial c^{\prime}$ does not coincide with the multiple intersection of two or more hyperplanes. Thus, we need to show that $c_{ \pm \varepsilon} \cap c^{\prime} \neq \emptyset$. Notice that $\partial c \cap \partial c^{\prime}=\widetilde{H}_{a b}$ for suitable $a, b$. Then, suppose without loss of generality that $P R_{a b}(t)$ crosses $l_{+}(\widetilde{\tau})$ clockwise when $t$ crosses $\widetilde{H}_{a b} \backslash \Delta$ moving along a curve from $c$ to $c^{\prime}$. An example of this crossing is the transition from figure 4 to figure 6 , with the identification 


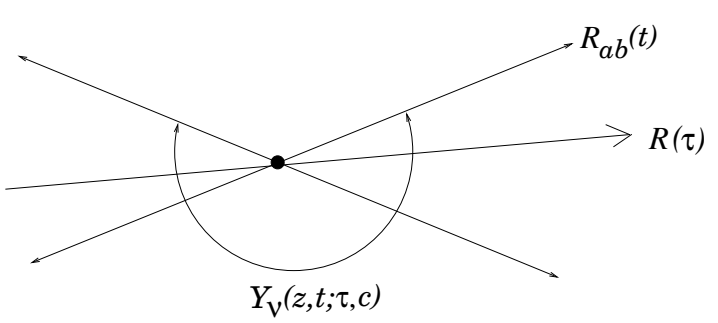

Figure 9. $Y_{\nu}(z, t ; \widetilde{\tau}, c)$ for $t \in c$. The sector where $Y_{\nu}(z, t ; \tilde{\tau}, c)$ has the canonical asymptotic behaviour is represented.

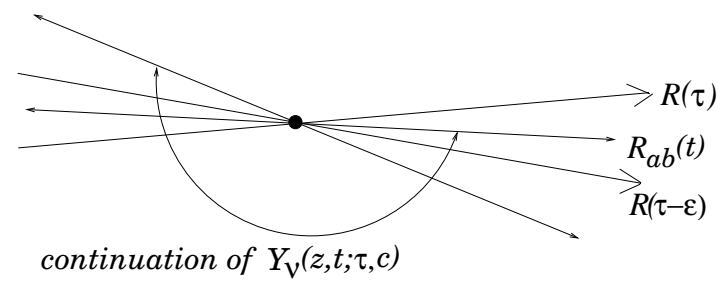

Figure 10. Analytic continuation of $Y_{\nu}(z, t ; \widetilde{\tau}, c)$ for $t$ in the neighbouring cell $c^{\prime}$ just after the crossing of $\partial c \backslash \Delta$, namely just after $R_{a b}(t)$ has crossed $R(\widetilde{\tau})$. The sector where $Y_{\nu}(z, t ; \widetilde{\tau}, c)$ has the canonical asymptotic behaviour is represented.

$c^{\prime}=c_{3}$ of Figure 11, and $P R_{1}=P R_{a b}$. Then, for the small deformation $\widetilde{\tau} \mapsto \widetilde{\tau}-\varepsilon$ the above discussion applies. Namely, $c_{-\epsilon} \cap c^{\prime} \neq \emptyset$. See figures 9 and 10 .

If $\partial c \cap \partial c^{\prime}=\widetilde{H}_{a b} \cap \widetilde{H}_{a^{\prime} b^{\prime}}$ for some $\left(a^{\prime}, b^{\prime}\right) \neq(a, b)$, there is multiple crossing of $l(\widetilde{\tau})$. The proof does not work if the crossing corresponds to a transition such as that from figure 4 to figure 7 , with the identification $c^{\prime}=c_{1}$. Since $P R_{1}$ and $P R_{2}$ cross simultaneously $l_{+}(\widetilde{\tau})$ from opposite sides, any deformation $\widetilde{\tau} \mapsto \widetilde{\tau} \pm \varepsilon$ produces a cell $c_{ \pm \varepsilon}$ which does not intersect $c_{1}$. In other words, the deformation prevents points of $c_{ \pm \varepsilon}$ from getting close to $\widetilde{H}_{a b} \cap \widetilde{H}_{a^{\prime} b^{\prime}}$. The schematic figure 11 shows the 4 cells corresponding to the figures from 4 to 7 . It is shown that $Y_{\nu}(z, t ; \widetilde{\tau}, c)$ can be continued slightly inside $c_{2}$ and $c_{3}$, but not inside $c^{\prime}=c_{1}$. It is worth noticing that both $Y_{\nu}\left(z, t ; \tilde{\tau}, c_{2}\right)$ and $Y_{\nu}\left(z, t ; \widetilde{\tau}, c_{3}\right)$ can be continued beyond $\widetilde{H}_{a b} \cap \widetilde{H}_{a^{\prime} b^{\prime}}$. See figure 12 for $Y_{\nu}\left(z, t ; \widetilde{\tau}, c_{3}\right)$.

Remark 13.2. If the eigenvalues are linear in $t$ as in (1.15), the results of this section assures that the fundamental solutions $Y_{\nu+k \mu}(z, t ; \widetilde{\tau}, c)$ 's are holomorphic in a $\widetilde{\tau}$-cell $c$ and a little beyond, that they maintain the asymptotic behaviour, and then the corresponding Stokes matrices $\mathbb{S}_{\nu+k \mu}(t)$ 's are defined and holomorphic in the whole $\widetilde{\tau}$-cell $c$ and a little bit beyond.

\section{Fundamental Solutions $Y_{\nu}(z, t)$ and Stokes Matrices $\mathbb{S}_{\nu}(t)$ Holomorphic At $\Delta$}

If the fundamental solutions $Y_{\nu+k \mu}(z, t ; \tilde{\tau}, c)$ 's of (2.1) (with Assumption 1) have analytic continuation to the whole $\mathcal{U}_{\epsilon_{0}}(0)$, in this section we give sufficient conditions such that the continuations are $c$ indendent solutions $Y_{\nu+k \mu}(z, t)$ 's, which maintain the asymptotic behaviour in large sectors $\widehat{\mathcal{S}}_{\nu}$ defined below, so that the Stokes matrices $\mathbb{S}_{\nu+k \mu}(t)$ are well defined in the whole $\mathcal{U}_{\epsilon_{0}}(0)$. Moreover, we show that $Y_{\nu+k \mu}(z, 0) \equiv \stackrel{\circ}{Y}_{\nu+k \mu}(z)$ and $\mathbb{S}_{\nu+k \mu}(0) \equiv \stackrel{\circ}{\mathbb{S}}_{\nu+k \mu}$, where $\stackrel{\circ}{Y+k \mu}_{\nu}(z), \stackrel{\circ}{\mathbb{S}}_{\nu+k \mu}$ have been defined in Section 6 for the system at fixed $t=0$.

14.1. Restriction of $\epsilon_{0}$. So far, $\epsilon_{0}$ has been taken so small that $\Lambda_{i}(t)$ and $\Lambda_{j}(t), 1 \leq i \neq j \leq s$, have no common eigenvalues for $t \in \mathcal{U}_{\epsilon_{0}}(0)$. If $\Lambda=\Lambda(0)$ has at least two distinct eigenvalues, we consider a further restriction of $\epsilon_{0}$. Let $\widetilde{\eta}=3 \pi / 2-\widetilde{\tau}$ be the admissible direction associated with the direction $\widetilde{\tau}$ of the admissible ray $R(\widetilde{\tau})$. Let $\delta_{0}$ be a small positive number such that

$$
\delta_{0}<\min _{1 \leq i \neq j \leq s} \delta_{i j}
$$

where $\delta_{i j}$ is $1 / 2$ of the distance between two parallel lines of angular direction $\widetilde{\eta}$ in the $\lambda$-plane, one passing through $\lambda_{i}$ and one through $\lambda_{j}$; namely

$$
\delta_{i j}:=\frac{1}{2} \min \left\{\left|\lambda_{i}-\lambda_{j}+\rho e^{i \tilde{\eta}}\right|, \rho \in \mathbb{R}\right\}, \quad i \neq j=1,2, \ldots, s .
$$

Clearly, $\delta_{0}$ depends on the choice of $\widetilde{\eta}$ (see also Remark 14.2). Let $\bar{B}\left(\lambda_{i} ; \delta_{0}\right)$ be the closed ball in $\mathbb{C}$ with center $\lambda_{i}$ and radius $\delta_{0}$. Then, we choose $\epsilon_{0}$ so small that the eigenvalues $u_{1}(t), \ldots, u_{n}(t)$ for $t \in \mathcal{U}_{\epsilon_{0}}(0)$ 


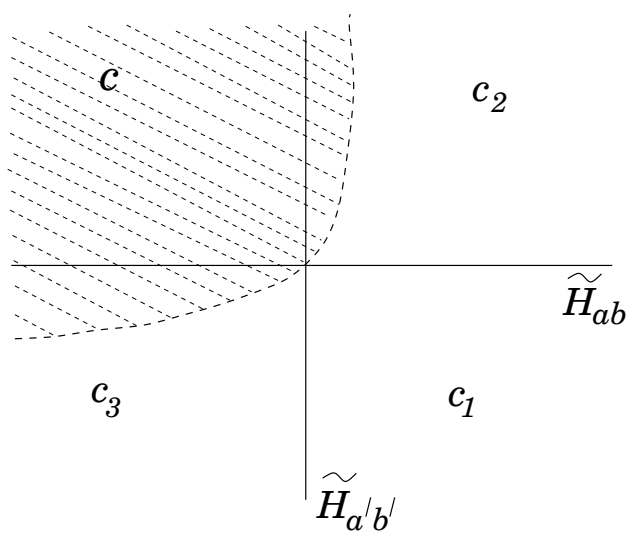

Figure 11. The cells of complex dimension $n$ (real dimension $2 n$ ) are schematically and improperly depicted in real dimension 2. Boundaries $\widetilde{H}_{a b}$ and $\widetilde{H}_{a^{\prime} b^{\prime}}$ are represented as lines, their intersection as a point (understanding that it is not in $\Delta$ ). The domain of the analytic continuation of $Y_{\nu}(z, t ; \widetilde{\tau}, c)$ beyond the boundary of $c$ is the dashed region. The analytic continuation does not go beyond $\widetilde{H}_{a b} \cap \widetilde{H}_{a^{\prime} b^{\prime}}$, because the transition from figure 4 to figure 7 is obtained by a simultaneous crossing of $l(\widetilde{\tau})$ by $P R_{1}$ and $P R_{2}$ from opposite sides of $l(\widetilde{\tau})$.

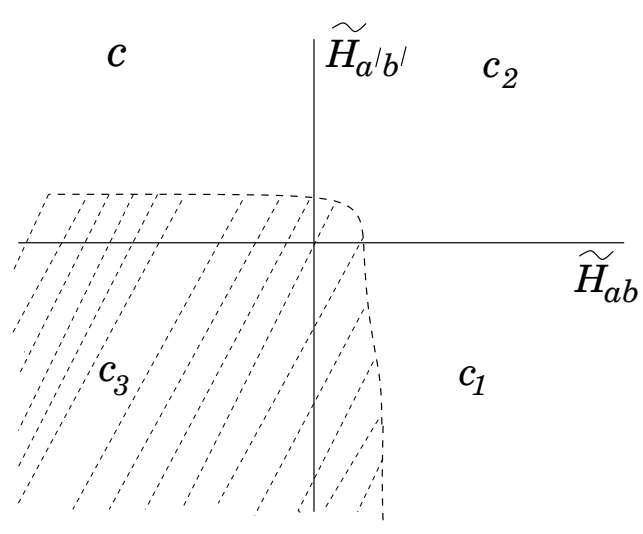

Figure 12. Analytic continuation of $Y_{\nu}\left(z, t ; \widetilde{\tau}, c_{3}\right)$ beyond the boundary of $c$. The continuation goes up to the 3 neighbouring cells. This corresponds to the fact that the three transitions form figure 6 to figures 4 and 7 occur when $P R_{1}$ and $P R_{2}$ respectively cross $l(\tau)$, while the transition from figure 6 to figure 5 occurs when $P R_{1}$ and $P R_{2}$ simultaneously cross $l(\tau)$, coming from the same side of $l(\widetilde{\tau})$ (moving in anticlockwise sense).

satisfy

$$
\left(u_{1}(t), \ldots, u_{n}(t)\right) \in \bar{B}\left(\lambda_{1} ; \delta_{0}\right)^{\times p_{1}} \times \cdots \times \bar{B}\left(\lambda_{s} ; \delta_{0}\right)^{\times p_{s}} .
$$

As $t$ varies in $\mathcal{U}_{\epsilon_{0}}(0)$ above, the Stokes rays continuously move, but the directions of the rays associated with a $u_{a} \in \bar{B}\left(\lambda_{i} ; \delta_{0}\right)$ and a $u_{b} \in \bar{B}\left(\lambda_{j} ; \delta_{0}\right), i \neq j$, never cross the values $\widetilde{\eta}$ and $\widetilde{\eta}-\pi(\bmod 2 \pi)$, so that the projected rays $P R_{a b}(t)$ and $P R_{b a}(t)$ never cross the admissible line $l(\widetilde{\tau})$. It follows that

the cell decomposition only depends on the Stokes rays associated with couples $\left(u_{a}(t), u_{b}(t)\right)$ such that $u_{a}(0)=u_{b}(0)=\lambda_{i}, i=1, \ldots, s$.

For eigenvalues linear in $t$ as in (1.15), we can take $\epsilon_{0}=\delta_{0}$ and

$$
\mathcal{U}_{\epsilon_{0}}(0) \equiv \bar{B}\left(0 ; \delta_{0}\right)^{\times p_{1}} \times \cdots \times \bar{B}\left(0 ; \delta_{0}\right)^{\times p_{s}}, \quad \epsilon_{0}=\delta_{0} .
$$

Remark 14.1. If $t$ moves from one $\widetilde{\tau}$-cell to another, the only Stokes rays which may cross admissible rays $R(\widetilde{\tau}+k \pi), k \in \mathbb{Z}$, are those associated with pairs $u_{a}(t), u_{b}(t)$ with $u_{a}(0)=u_{b}(0)=\lambda_{i}, i=$ $1, \ldots, s$. Therefore, the boundaries of the cells are only the $\widetilde{H}_{a b}$ 's such that $u_{a}(0)=u_{b}(0)$. In this case, 

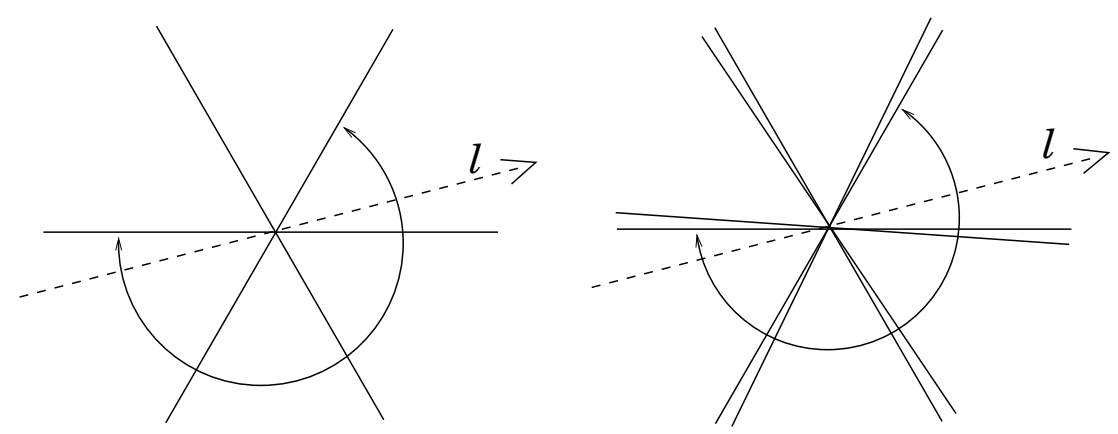

Figure 13. In the left figure $t=0$ and the sector $\mathcal{S}_{\nu}$ is represented. The explanation is as for the left part of Figure 2. In the right figure, $t \neq 0$. Represented are only the rays associated with couples $u_{a}(t), u_{b}(t)$ with $u_{a}(0)=\lambda_{i}, u_{b}(0)=\lambda_{j}$, for $i \neq j$, together with the sector $\widehat{\mathcal{S}}_{\nu}(t)$.

$L_{a b}\left(\mathbf{x}_{0}, \mathbf{y}_{0}\right)=0$, so that

$$
H_{a b}^{\prime}:=\left\{(\Re t, \Im t) \in \mathbb{R}^{2 n} \mid L_{a b}(\Re t, \Im t)=0\right\} .
$$

Remark 10.3 follows from the above observations.

14.2. The Sectors $\widehat{\mathcal{S}}_{\nu}(t)$ and $\widehat{\mathcal{S}}_{\nu}$. Let $\Lambda(t)$ be of the form (1.2) with eigenvalues (1.15). Let $\epsilon_{0}=\delta_{0}$ be as in subsection 14.1. We define a subset $\mathfrak{R}(t)$ of the set of Stokes rays of $\Lambda(t)$ as follows: $\mathfrak{R}(t)$ contains only those Stokes rays $\left\{z \in \mathcal{R} \mid \Re\left(z\left(u_{a}(t)-u_{b}(t)\right)\right)=0\right\}$ which are associated with pairs $u_{a}(t), u_{b}(t)$ satisfying the condition $u_{a}(0) \neq u_{b}(0)$ (namely, $u_{a}(0)=\lambda_{i}, u_{b}(0)=\lambda_{j}, i \neq j$; see (5.1)-(5.4)). The reader may visualise the rays in $\mathfrak{R}(t)$ as being originated by the splitting of Stokes rays of $\Lambda(0)$. See figure 13.

$\mathfrak{R}(t)$ has the following important property: if $t$ varies in $\mathcal{U}_{\epsilon_{0}}(0)$, the rays in $\mathfrak{R}(t)$ continuously move, but since $\epsilon_{0}=\delta_{0}$, they never cross any admissible ray $R(\widetilde{\tau}+k \pi), k \in \mathbb{Z}$.

Definition 14.1 (Sectors $\left.\widehat{\mathcal{S}}_{\nu+k \mu}(t)\right)$. We define $\widehat{\mathcal{S}}_{\nu+k \mu}(t)$ to be the unique sector containing $S(\widetilde{\tau}-\pi+$ $k \pi, \widetilde{\tau}+k \pi)$ and extending up to the nearest Stokes rays in $\mathfrak{R}(t), t \in \mathcal{U}_{\epsilon_{0}}(0)$.

Any $\widehat{\mathcal{S}}_{\nu+k \mu}(t)$ contains a set of basic Stokes rays of $\mathfrak{R}$. Moreover,

$$
R(\widetilde{\tau}) \subset \widehat{\mathcal{S}}_{\nu}(t) \cap \widehat{\mathcal{S}}_{\nu+\mu}(t) \subset S\left(\tau_{\nu}, \tau_{\nu+1}\right),
$$

and

$$
\mathcal{S}_{\nu}(t) \subset \widehat{\mathcal{S}}_{\nu}(t), \quad \widehat{\mathcal{S}}_{\nu}(0) \equiv \mathcal{S}_{\nu}
$$

In case $\Lambda(0)=\lambda_{1} I$, then $\widehat{\mathcal{S}}_{\nu}(t)$ is unbounded, namely it coincides with $\mathcal{R}$.

Definition 14.2 (Sectors $\widehat{\mathcal{S}}_{\nu}(K)$ ). For any compact $K \subset \mathcal{U}_{\epsilon_{0}}(0)$ we define

$$
\widehat{\mathcal{S}}_{\nu}(K):=\bigcap_{t \in K} \widehat{\mathcal{S}}_{\nu}(t)
$$

If $K_{1} \subset K_{2}$, then $\widehat{\mathcal{S}}_{\nu}\left(K_{2}\right) \subset \widehat{\mathcal{S}}_{\nu}\left(K_{1}\right)$. For any $K_{1}, K_{2}$, we have $\widehat{\mathcal{S}}_{\nu}\left(K_{1} \cup K_{2}\right)=\widehat{\mathcal{S}}_{\nu}\left(K_{1}\right) \cap \widehat{\mathcal{S}}_{\nu}\left(K_{2}\right)$.

Definition 14.3 (Sectors $\widehat{\mathcal{S}}_{\nu}$ ). If $K=\mathcal{U}_{\epsilon_{0}}(0)$, we define

$$
\widehat{\mathcal{S}}_{\nu}:=\widehat{\mathcal{S}}_{\nu}\left(\mathcal{U}_{\epsilon_{0}}(0)\right) \text {. }
$$

Since $\epsilon_{0}=\delta_{0}, \widehat{\mathcal{S}}_{\nu}$ has angular opening greater than $\pi$ and

$$
\begin{aligned}
& \widehat{\mathcal{S}}_{\nu} \subset \widehat{\mathcal{S}}_{\nu}(0) \equiv \mathcal{S}_{\nu}, \\
& R(\widetilde{\tau}) \subset \widehat{\mathcal{S}}_{\nu} \cap \widehat{\mathcal{S}}_{\nu+\mu} \subset S\left(\tau_{\nu}, \tau_{\nu+1}\right) .
\end{aligned}
$$

Remark 14.2. Notice that $\widetilde{\tau} \in\left(\tau_{\nu}, \tau_{\nu+1}\right)$ determines $\delta_{0}$ through (14.2) and (14.1). Let $\widetilde{\tau}^{\prime} \in\left(\tau_{\nu}, \tau_{\nu+1}\right)$ and let $\delta_{0}^{\prime}$ be obtained through (14.2) and (14.1). Let $\epsilon_{0}=\min \left\{\delta_{0}, \delta_{0}^{\prime}\right\}$. We temporarily denote by $\widehat{\mathcal{S}}_{\nu}[\widetilde{\tau}]$ the sector $\widehat{\mathcal{S}}_{\nu}$ of Definition 14.3 obtained starting from $\widetilde{\tau}$. Then for the above $\epsilon_{0}$ we have

$$
\widehat{\mathcal{S}}_{\nu}[\widetilde{\tau}]=\widehat{\mathcal{S}}_{\nu}\left[\widetilde{\tau}^{\prime}\right] .
$$




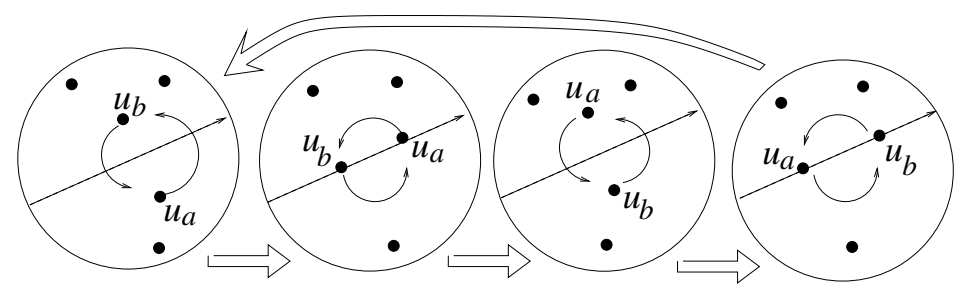

FiguRE 14. Loop $\gamma_{a b}$ represented in $\bar{B}\left(\lambda_{i} ; \epsilon_{0}\right)$. The dashed oriented line is the direction $\widetilde{\eta}$. Along the loop, $u_{a}$ and $u_{b}$ get aligned with $\widetilde{\eta}$ twice, in the second and fourth figures. The second figure corresponds to the passage from one initial cell $c$ to a neighbouring cell $c^{\prime}$ (while $P R_{a b}$ crosses clockwise a half line of $l(\widetilde{\tau})$ ) and the fourth figure to the return to $c$ (while $P R_{a b}$ crosses clockwise the opposite half line of $l(\widetilde{\tau})$ ). Other dots represent other eigenvalues $u_{\gamma}(t)$ in $\bar{B}\left(\lambda_{i} ; \epsilon_{0}\right)$.

14.3. Fundamental group of $\mathcal{U}_{\epsilon_{0}}(0) \backslash \Delta$ and generators. Let the eigenvalues of $\Lambda(t)$ be linear in $t$ as in $(1.15), \tau_{\nu}<\widetilde{\tau}<\tau_{\nu+1}$ and $\widetilde{\eta}=3 \pi / 2-\widetilde{\tau}$.

The fundamental group $\pi_{1}\left(\mathcal{U}_{\epsilon_{0}}(0) \backslash \Delta, t_{\text {base }}\right)$ is generated by loops $\gamma_{a b}, 1 \leq a \neq b \leq n$, which are homotopy classes of simple curves encircling the component $\left\{t \in \mathcal{U}_{\epsilon_{0}}(0) \mid u_{a}(t)=u_{b}(t)\right\}$ of $\Delta$. The choice of the base point is free, because $\mathcal{U}_{\epsilon_{0}}(0) \backslash \Delta$ is path-wise connected, since $\Delta$ is a braid arrangement in $\mathcal{U}_{\epsilon_{0}}(0)$ and the hyperplanes are complex.

For $\epsilon_{0}=\delta_{0}$ of Section 14.1, Stokes rays in $\mathfrak{R}(t)$ never cross the admissible rays $R(\widetilde{\tau}+k \pi), k \in \mathbb{Z}$, when $t$ goes along any loop in $\mathcal{U}_{\epsilon_{0}}(0)$ (see Remark 14.1). Therefore, as far as the analytic continuation of $Y_{\nu}(z, t)$ is concerned, it is enough to consider $u_{a}(t)$ and $u_{b}(t)$ coming from the unfolding of an eigenvalue $\lambda_{i}$ of $\Lambda(0)$ (see the beginning of Section 9), namely

$$
u_{a}(t)=\lambda_{i}+t_{a}, \quad u_{b}(t)=\lambda_{i}+t_{b}
$$

If we represent $t_{a}$ and $t_{b}$ in the same complex plane, so that $t_{a}-t_{b}$ is a complex number, a representative of $\gamma_{a b}$, which we also denote $\gamma_{a b}$ with abuse of notation, is represented by the following loop around $t_{a}-t_{b}=0$,

$$
t_{a}-t_{b} \longmapsto\left(t_{a}-t_{b}\right) e^{2 \pi i}
$$

$\left|t_{a}-t_{b}\right|$ will be taken small. The Stokes rays associated with $u_{a}(t)$ and $u_{b}(t)$ have directions

$$
\frac{3 \pi}{2}-\arg \left(t_{a}-t_{b}\right) \quad \bmod (2 \pi), \quad \frac{3 \pi}{2}-\arg \left(t_{b}-t_{a}\right) \bmod (2 \pi) .
$$

The projection of these rays onto $\mathbb{C}$ are the two opposite rays $P R_{a b}(t)$ and $P R_{b a}(t)$, as in (13.2) . Along the loop (14.5), each of these rays rotate clockwise and crosses the line $l(\widetilde{\tau})$ twice (recall Definition 6.2), once passing over the positive half line and once over the negative half line, returning to the initial position at the end of the loop. Hence, the support of $\gamma_{a b}$ is contained in at least two cells, but generally in more than two, as follows.

- There exists a representative contained in only two cells if only $P R_{a b}(t)$ and its opposite $P R_{b a}(t)$ cross $l(\widetilde{\tau})$, each twice. For example, in figure 14 the ball $\bar{B}\left(\lambda_{i} ; \epsilon_{0}\right)$ is represented with the loop (14.5). The dots represent other points $u_{\gamma}(t) \in \bar{B}\left(\lambda_{i} ; \epsilon_{0}\right), \gamma \neq a, b . P R_{a b}(t)$ and $P R_{b a}(t) \operatorname{cross} l(\widetilde{\tau})$ when $u_{a}(t)$ and $u_{b}(t)$ are aligned with the admissible direction $\widetilde{\eta}$. Along the loop, no other $u_{\gamma}$ aligns with $u_{a}(t)$ and $u_{b}(t)$.

- In general, other (projected) rays cross $l(\widetilde{\tau})$ along any possible representative of $\gamma_{a b}$. For example, the representative of (14.5) in figure 15 is contained in three cells. Indeed, also $P R_{a \gamma}(t)$ and $P R_{\gamma a}(t)$ $\operatorname{cross} l(\widetilde{\tau})$ when $u_{a}$ and $u_{\gamma}$ get aligned with $\widetilde{\eta}$. Alignment corresponds to the passage from one cell to another.

14.4. Holomorphic conditions such that $Y_{\nu}(z, t) \rightarrow \stackrel{\circ}{Y}_{\nu}(z)$ and $\mathbb{S}_{\nu}(t) \rightarrow \stackrel{\circ}{\mathbb{S}}_{\nu}$ for $t \rightarrow 0$, in case of linear eigenvalues (1.15). The following theorem is one of the central results of the paper, and it will be used to prove Theorem 1.1. 


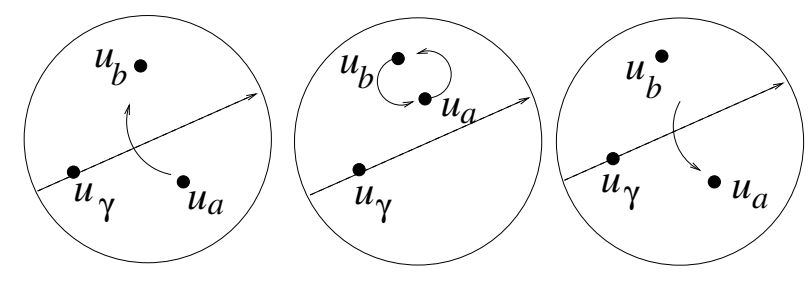

Figure 15. Loop $\gamma_{a b}$ represented in $\bar{B}\left(\lambda_{i} ; \epsilon_{0}\right)$. The dashed oriented line is the direction $\widetilde{\eta}$. In the first figure, $u_{a}$ moves close to $u_{b}$. Along the way it gets aligned with $u_{\gamma}$. At this alignment, $P R_{a \gamma}$ crosses clockwise a half line of $l(\widetilde{\tau})$ and $t$ passes from the initial cell $c$ to a cell $c^{\prime}$. The second figure is figure 14. Here $t$ passes from $c^{\prime}$ to another cell $c^{\prime \prime}$ and then back to $c^{\prime}$. In the third figure, $u_{a}$ moves to the initial position. Along the way it gets aligned with $u_{\gamma}, P R_{a \gamma}$ crosses anti-clockwise the same half line of $l(\widetilde{\tau})$ and $t$ returns to the cell $c$. In this example, $\gamma_{a b}$ has support contained in three cells.

Theorem 14.1. Consider the system (2.1) (i.e. system (1.1) of the Introduction). Let Assumption 1 hold, so that (2.1) is holomorphically equivalent to the system (5.18) (i.e. to (1.5) of the Introduction). Let $\Lambda(t)$ be of the form (1.2), with eigenvalues (1.15) and $\epsilon_{0}=\delta_{0}$ as in subsection 14.1. Let $\widetilde{\tau}$ be the direction of an admissible ray $R(\widetilde{\tau})$, satisfying $\tau_{\nu}<\widetilde{\tau}<\tau_{\nu+1}$. Suppose that:

1) For every integer $j \geq 1$, the $F_{j}(t)$ 's are holomorphic in $\mathcal{U}_{\epsilon_{0}}(0)$ (so necessary and sufficient conditions of Proposition 5.1 hold);

2) For any $\widetilde{\tau}$-cell $c$ of $\mathcal{U}_{\epsilon_{0}}(0)$ and any $k \in \mathbb{Z}$, the fundamental solution $Y_{\nu+k \mu}(z, t ; \widetilde{\tau}, c)$ has analytic continuation as a single-valued holomorphic function on the whole $\mathcal{U}_{\epsilon_{0}}(0)$. Denote the analytic continuation with the same symbol $Y_{\nu+k \mu}(z, t ; \widetilde{\tau}, c), t \in \mathcal{U}_{\epsilon_{0}}(0)$.

Then:

- For any $\tilde{\tau}$-cells $c$ and $c^{\prime}$,

$$
Y_{\nu+k \mu}(z, t ; \widetilde{\tau}, c)=Y_{\nu+k \mu}\left(z, t ; \widetilde{\tau}, c^{\prime}\right), \quad t \in \mathcal{U}_{\epsilon_{0}}(0) .
$$

Therefore, we can simply write $Y_{\nu+k \mu}(z, t ; \widetilde{\tau})$.

- Let $\mathcal{G}_{\nu+k \mu}(z, t ; \widetilde{\tau}):=G_{0}(t)^{-1} Y_{\nu+k \mu}(z, t ; \widetilde{\tau}) z^{-B_{1}(t)} e^{-\Lambda(t) z}$. For any $\epsilon_{1}<\epsilon_{0}$ the following asymptotic expansion holds:

$$
\mathcal{G}_{\nu+k \mu}(z, t ; \widetilde{\tau}) \sim I+\sum_{k=0}^{\infty} F_{k}(t) z^{-k}, \quad z \rightarrow \infty, \quad z \in \widehat{\mathcal{S}}_{\nu+k \mu}, \quad t \in \mathcal{U}_{\epsilon_{1}}(0) .
$$

The asymptotic expansion is uniform in $t$ in $\mathcal{U}_{\epsilon_{1}}(0)$ and uniform in $z$ in any closed subsector of $\widehat{\mathcal{S}}_{\nu+k \mu}$.

- For any $t \in \mathcal{U}_{\epsilon_{1}}(0)$, the diagonal blocks of any Stokes matrix $\mathbb{S}_{\nu+k \mu}(t)$ are the identity matrices $I_{p_{1}}$, $I_{p_{2}}, \ldots, I_{p_{s}}$. Namely

$$
\left(\mathbb{S}_{\nu+k \mu}\right)_{a b}(t)=\left(\mathbb{S}_{\nu+k \mu}\right)_{b a}(t)=0 \quad \text { whenever } u_{a}(0)=u_{b}(0)
$$

Remark 14.3. [Continuation of Remark 14.2] Since $Y_{\nu+k \mu}(z, t ; \widetilde{\tau}, c)=Y_{\nu+k \mu}\left(z, t ; \widetilde{\tau}, c^{\prime}\right) \equiv Y_{\nu+k \mu}(z, t ; \widetilde{\tau})$, only the choice of $\widetilde{\tau}$ is relevant. If $\widetilde{\tau}$ and $\widetilde{\tau}^{\prime}$ are as in Remark 14.2 , then

$$
Y_{\nu+k \mu}(z, t ; \widetilde{\tau})=Y_{\nu+k \mu}\left(z, t ; \widetilde{\tau}^{\prime}\right)
$$

because the rays in $\mathfrak{R}(t), t \in \mathcal{U}_{\epsilon_{0}}(0)$, neither cross the admissible rays $R(\widetilde{\tau}+m \pi)$ nor the rays $R\left(\widetilde{\tau}^{\prime}+m \pi\right)$, $m \in \mathbb{Z}$. In other words, $Y_{\nu+k \mu}(z, t ; \widetilde{\tau})$ depends on $\widetilde{\tau}$ only through $\epsilon_{0}$. Hence, we can restore the notation

$$
Y_{\nu+k \mu}(z, t), \quad t \in \mathcal{U}_{\epsilon_{0}}(0) .
$$


Corollary 14.1. Let the assumptions of Theorem 14.1 hold. Let $\stackrel{\circ}{Y}_{\nu+k \mu}(z), k \in \mathbb{Z}$, denote the unique fundamental solution (6.6) of the form (4.20), namely

$$
\stackrel{\circ}{Y}_{\nu+k \mu}(z)=\mathcal{G}_{\nu+k \mu}(z) z^{B_{1}(0)} e^{\Lambda z}
$$

with the asymptotics (4.21)

$$
\mathcal{G}_{\nu+k \mu}(z) \sim I+\sum_{j=1}^{\infty} \stackrel{\circ}{F}_{j} z^{-j}, \quad z \rightarrow \infty, \quad z \in \mathcal{S}_{\nu+k \mu},
$$

corresponding to the particular choice $\stackrel{\circ}{F}_{j}=F_{j}(0), j \geq 1$. Then,

$$
\begin{aligned}
& \mathcal{G}_{\nu+k \mu}(z, 0)=\mathcal{G}_{\nu+k \mu}(z), \\
& Y_{\nu+k \mu}(z, 0)=\stackrel{\circ}{Y}_{\nu+k \mu}(z) .
\end{aligned}
$$

Proof: Observe that $Y_{\nu+k \mu}(z, 0)$ is defined at $t=0$. Now, $\widehat{\mathcal{S}}_{\nu} \subset \mathcal{S}_{\nu}$ and both sectors have central opening angle greater than $\pi$. Hence, the solution with given asymptotics in $\widehat{\mathcal{S}}_{\nu}$ is unique, namely $\mathcal{G}_{\nu}(z)=\mathcal{G}_{\nu}(z, 0)$.

Corollary 14.2. Let the assumptions of Theorem 14.1 hold. Let $\mathbb{S}_{\nu}(t), \mathbb{S}_{\nu+\mu}(t)$ be a complete set of Stokes matrices associated with fundamental solutions $Y_{\nu}(z, t), Y_{\nu+\mu}(z, t), Y_{\nu+2 \mu}(z, t)$, with canonical asymptotics, for $t$ in a $\widetilde{\tau}$-cell of $\mathcal{U}_{\epsilon_{0}}(0)$, in sectors $\mathcal{S}_{\nu}(t), \mathcal{S}_{\nu+\mu}(t)$ and $\mathcal{S}_{\nu+2 \mu}(t)$ respectively, which by Theorem 14.1 extend to $\widehat{\mathcal{S}}_{\nu}, \widehat{\mathcal{S}}_{\nu+\mu}$ and $\widehat{\mathcal{S}}_{\nu+2 \mu}$ respectively for $t \in \mathcal{U}_{\epsilon_{1}}(0)$. Then there exist

$$
\lim _{t \rightarrow 0} \mathbb{S}_{\nu}(t)=\stackrel{\circ}{\mathbb{S}}_{\nu}, \quad \lim _{t \rightarrow 0} \mathbb{S}_{\nu+\mu}(t)=\stackrel{\circ}{\mathbb{S}}_{\nu+\mu}
$$

where $\stackrel{\circ}{S}_{\nu}, \stackrel{\circ}{S}_{\nu+\mu}$ is a complete set of Stokes matrices for the system at $t=0$, referred to three fundamental

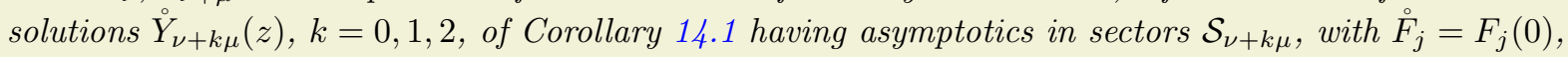
$j \geq 1$.

Proof: The analyticity of $Y_{\nu+k \mu}(z, t)$ in assumption 2) of Theorem 14.1 implies that the Stokes matrices are holomorphic in $\mathcal{U}_{\epsilon_{0}}(0)$. Hence, for $k=1,2$, there exists

$$
\mathbb{S}_{\nu+k \mu}(0)=\lim _{t \rightarrow 0}\left(Y_{\nu+(k+1) \mu}(z, t)^{-1} Y_{\nu+k \mu}(z, t)\right)=\stackrel{\circ}{Y}_{\nu+(k+1) \mu}(z)^{-1} \stackrel{\circ}{Y+k \mu}_{\nu}(z)=\stackrel{\circ}{\mathbb{S}}_{\nu+k \mu}
$$

\subsubsection{Proof of Theorem 14.1.}

Lemma 14.1. Let Assumption 1 hold for the system (2.1). Let the eigenvalues of $\Lambda(t)$ be linear in $t$ as in (1.15). Suppose that $Y_{\nu}(z, t ; \widetilde{\tau}, c)$ has t-analytic continuation on $\mathcal{U}_{\epsilon_{0}}(0) \backslash \Delta$, with $\epsilon_{0}=\delta_{0}$ as in subsection 14.1. Temporarily call $Y_{\nu}^{\text {cont }}(z, t ; \widetilde{\tau}, c)$ the continuation. Also suppose that

$$
\left.Y_{\nu}^{\text {cont }}(z, t ; \widetilde{\tau}, c)\right|_{t \in c^{\prime}}=Y_{\nu}\left(z, t ; \widetilde{\tau}, c^{\prime}\right)
$$

Then:

a) Any $Y_{\nu}\left(z, t ; \widetilde{\tau}, c^{\prime}\right)$ has analytic continuation on $\mathcal{U}_{\epsilon_{0}}(0) \backslash \Delta$, coinciding with $Y_{\nu}^{\text {cont }}(z, t ; \widetilde{\tau}, c)$. Due to the independence of $c$, we denote this continuation by

$$
Y_{\nu}(z, t ; \widetilde{\tau})
$$

b) $\mathcal{G}_{\nu}(z, t ; \widetilde{\tau}):=G_{0}(t)^{-1} Y_{\nu}(z, t ; \widetilde{\tau}) z^{-B_{1}(t) z} e^{-\Lambda(t) z}$ has asymptotic expansion

$$
\mathcal{G}_{\nu}(z, t ; \widetilde{\tau}) \sim I+\sum_{k=0}^{\infty} F_{k}(t) z^{-k}, \quad z \rightarrow \infty, \quad z \in \widehat{\mathcal{S}}_{\nu}(t), \quad t \in \mathcal{U}_{\epsilon_{0}}(0) \backslash \Delta .
$$

The asymptotics for $z \rightarrow \infty$ in $\widehat{\mathcal{S}}_{\nu}(K)$ is uniform on any compact subset $K \in \mathcal{U}_{\epsilon_{0}}(0) \backslash \Delta$. 
Proof of Lemma 14.1: a) is obvious. We prove b), dividing the proof into two parts.

Part 1 (in steps). Chosen an arbitrary cell $c$ (all cells are equivalent, by a)) and any $\breve{t} \in c$, we prove that the sector where $Y_{\nu}(z, \breve{t} ; \widetilde{\tau})$ has canonical asymptotics can be extended from $\mathcal{S}_{\nu}(\breve{t})$ to $\widehat{\mathcal{S}}_{\nu}(\breve{t})$. For clarity in the discussion below, let us still write $Y_{\nu}(z, \breve{t} ; \widetilde{\tau}, c)$.

Step 1. At $\breve{t}$, consider the Stokes rays in $\widehat{\mathcal{S}}_{\nu}(\breve{t}) \backslash S(\widetilde{\tau}-\pi, \widetilde{\tau})$ associated with the unfolding of the $\lambda_{i}$ 's. Those with direction greater than $\widetilde{\tau}$ will be labelled in anticlockwise sense as $R_{1}(\breve{t}), R_{2}(\breve{t}), \ldots$, etc. Those with direction smaller than $\widetilde{\tau}-\pi$ will be labelled in clockwise sense $R_{1}^{\prime}(\breve{t}), R_{2}^{\prime}(\breve{t})$, etc. Therefore, $R_{1}(\breve{t})$ is the closest to the admissible ray $R(\widetilde{\tau})$, while $R_{1}^{\prime}(\breve{t})$ is the closest to $R(\widetilde{\tau}-\pi)$. (Warning about the notation: The dependence on $t$ is indicated in Stokes rays $R_{1}, R_{2}$ etc, while for the admissible ray $R(\widetilde{\tau})$, $\widetilde{\tau}$ is the direction as in Definition 6.2). See figure 16 .

Let $t$ vary from $\breve{t}$ into a neighbouring cell $c_{1}$, in such a way that $R_{1}(t)$ approaches and crosses $R(\widetilde{\tau})$ clockwise. By Proposition 13.3, $Y_{\nu}^{\text {cont }}(z, t ; \widetilde{\tau}, c)$ is well defined with canonical asymptotics on a sector having left boundary ray equal to $R_{1}(t)$, for values of $t \in c_{1}$ just after the crossing. ${ }^{19}$

By assumption, $Y_{\nu}^{\text {cont }}(z, t ; \widetilde{\tau}, c)=Y_{\nu}\left(z, t ; \widetilde{\tau}, c_{1}\right)$. For $t \in c_{1}$ just after the crossing, $Y_{\nu}\left(z, t ; \widetilde{\tau}, c_{1}\right)$ has canonical asymptotics in $\mathcal{S}_{\nu}(t)$, which now has left boundary ray equal to $R_{2}(t)$. See Figures 17 e 18 . This implies that $Y_{\nu}^{\text {cont }}(z, t ; \widetilde{\tau}, c)$ has canonical asymptotics extended up to $R_{2}(t), t \in c_{1}$ as above. See Figure 19.

Let $t$ go back along the same path, so that $R_{1}(t)$ crosses $R(\widetilde{\tau})$ anticlockwise. Proposition 13.3 now can be applied to $Y_{\nu}\left(z, t ; \widetilde{\tau}, c_{1}\right)$ for this crossing. ${ }^{20}$ Hence, $Y_{\nu}\left(z, t ; \widetilde{\tau}, c_{1}\right)$ has analytic continuation for $t$ before the crossing, certainly up to $\breve{t}$ (because $R_{1}(t)$ does not cross $R_{2}(t)$ ), with canonical asymptotics in a sector having $R_{2}(\breve{t})$ as left boundary. See Figure 20. Again, by assumption, we have that $Y_{\nu}(z, \breve{t ;} ; \widetilde{\tau}, c)=$ $Y_{\nu}^{\text {cont }}\left(z, \breve{t} ; \widetilde{\tau}, c_{1}\right)$. Hence, $Y_{\nu}(z, \breve{t} ; \widetilde{\tau}, c)$ has canonical asymptotics extended up to the ray $R_{2}(\breve{t})$. See Figure 21. In conclusion, $R_{1}(t)$ has been erased.

Step 2. We repeat the arguments analogous to those of Step 1 in order to erase $R_{2}(t)$. Let $t$ vary in such a way that $R_{1}(t)$, which is now a "virtual ray", crosses $R(\widetilde{\tau})$ clockwise, as in step 1. After the crossing, $t \in c_{1}$ and $Y_{\nu}^{\text {cont }}(z, t ; \widetilde{\tau}, c)=Y_{\nu}\left(z, t ; \widetilde{\tau}, c_{1}\right)$. Then, let $t$ vary in such a way that also $R_{2}(t)$ crosses $R(\widetilde{\tau})$ clockwise. See Figures 22, 23. Just after the crossing, $t$ belongs to another cell $c_{2}$ (clearly, $c_{2} \neq c$ and $c_{1}$; see Proposition 13.2).

The same discussion done at Step 1 for $Y_{\nu}(z, t ; \widetilde{\tau}, c)$ is repeated now for $Y_{\nu}\left(z, t ; \widetilde{\tau}, c_{1}\right)$. Indeed, $Y_{\nu}^{\text {cont }}\left(z, t ; \widetilde{\tau}, c_{1}\right)=Y_{\nu}\left(z, t ; \widetilde{\tau}, c_{2}\right)$, for $t \in c_{2}$ just after $R_{2}(t)$ has crossed $R(\widetilde{\tau})$. The conclusion, as before, is that $Y_{\nu}^{\text {cont }}\left(z, t ; \widetilde{\tau}, c_{1}\right)$ has canonical asymptotics extended up to $R_{3}(t)$ for $t \in c_{1}$. See Figure 24 .

Now, let $t$ go back along the same path up to $\breve{t}$. Also the virtual ray $R_{1}(t)$ comes to the initial position, and $Y_{\nu}(z, \breve{t} ; \widetilde{\tau}, c)=Y_{\nu}^{\text {cont }}\left(z, \breve{t} ; \widetilde{\tau}, c_{1}\right)=Y_{\nu}^{\text {cont }}\left(z, \breve{t} ; \widetilde{\tau}, c_{2}\right)$, with canonical asymptotics extended up to $R_{3}(\breve{t})$. See figure 25 .

Step 3. The discussion above can be repeated for all Stokes rays $R_{1}, R_{2}, R_{3}$, etc.

Step 4. Observe that the right boundary ray $R_{1}^{\prime}$ of the sector where $Y_{\nu}(z, t ; \widetilde{\tau}, c)$ has asymptotics is not affected by the above construction. Once the left boundary rays $R_{1}, R_{2}, \ldots$ have been erased, the same discussion must be repeated considering crossings of the admissible ray $R(\widetilde{\tau}-\pi)$ by the rays $R_{1}^{\prime}$, $R_{2}^{\prime}$, etc, as in figure 26 .

In conclusion, all rays $R_{1}, R_{2}, \ldots, R_{1}^{\prime}, R_{2}^{\prime}, \ldots$ from unfolding lying in $\widehat{\mathcal{S}}_{\nu}(\breve{t}) \backslash S(\widetilde{\tau}-\pi, \widetilde{\tau})$ are erased. Hence $Y_{\nu}(z, \breve{t} ; \widetilde{\tau}, c) \equiv Y_{\nu}(z, \breve{t} ; \widetilde{\tau})$ has canonical asymptotics extended up to the closest Stokes rays in $\mathfrak{R}(\breve{t})$ outside $S(\widetilde{\tau}-\pi, \widetilde{\tau})$, namely the asymptotics holds in $\widehat{\mathcal{S}}_{\nu}(\breve{t})$.

The above discussion can be repeated also if one of more rays among $R_{1}, R_{2}$, etc. is double (i.e. it corresponds to three eigenvalues) at $\breve{t}$, because as $t$ varies the rays unfold. Thus, the above discussion holds for any $\breve{t} \in c$ and any $c$. Therefore, $Y_{\nu}(z, t ; \widetilde{\tau})$ has asymptotics in $\widehat{\mathcal{S}}_{\nu}(t)$ for any $t$ belonging to the union of the cells. ${ }^{21}$

\footnotetext{
${ }^{19}$ As long as $R_{1}(t)$ does not reach another Stokes ray

${ }^{20}$ In the proof, deform $\widetilde{\tau} \mapsto \widetilde{\tau}+\varepsilon$.

${ }^{21}$ Namely, $t \in \mathcal{U}_{\epsilon_{0}}(0) \backslash(\Delta \cup X(\widetilde{\tau}))=\mathcal{U}_{\epsilon_{0}}(0) \backslash\left(\bigcup \widetilde{H}_{a b}\right), a, b$ from unfolding.
} 


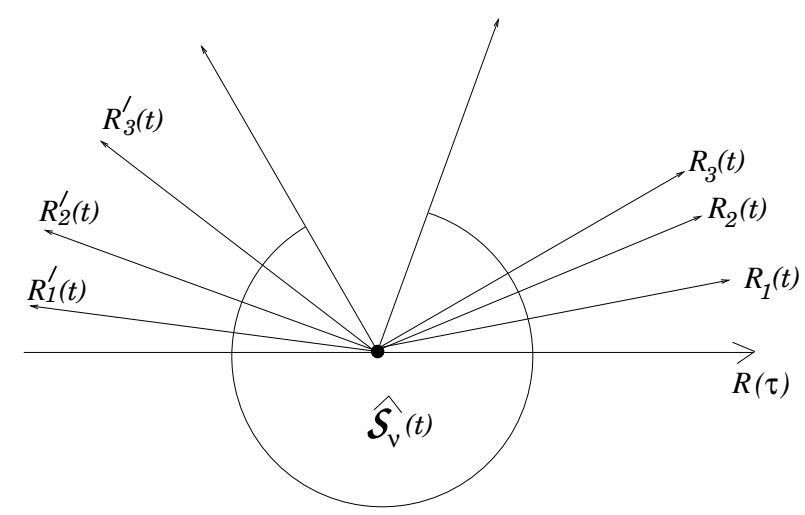

FiguRE 16. Rays in $\widehat{\mathcal{S}}_{\nu}(t)$ which are going to be erased in the proof.

We observe that a ray $R_{1}(t), R_{2}(t)$, etc, crosses $R(\widetilde{\tau})$ for $t$ equal to a simple point $t_{*}$ (see Definition 13.1). The above proof allows to conclude that $Y_{\nu}\left(z, t_{*} ; \widetilde{\tau}\right)$ has asymptotics in $\widehat{\mathcal{S}}_{\nu}\left(t_{*}\right)$ also when $\breve{t}=t_{*}$.

Part 2: Points $\breve{t}$ internal to cells and simple points have been considered. It remains to discuss non simple points $t_{*} \in\left(\widetilde{H}_{a_{1} b_{1}} \cap \widetilde{H}_{a_{2} b_{2}} \cap \cdots \cap \widetilde{H}_{a_{l}, b_{l}}\right) \backslash \Delta$, for some $l \geq 2$. Consider all the Stokes rays associated with either one of $\left(u_{a_{m}}(t), u_{b_{m}}(t)\right)$ or $\left(u_{b_{m}}(t), u_{a_{m}}(t)\right), m=1, \ldots, l$, and lying in $S(\widetilde{\tau}, \widetilde{\tau}+\pi)$. There exists a cell $c$, among the cells having boundary sharing the above intersection, such that these rays cross $R(\widetilde{\tau})$ clockwise and simultaneously at $t_{*}$, when $t$ approaches $t_{*}$ from $c$. Call these rays $R_{a_{1} b_{1}}(t), R_{a_{2} b_{2}}(t)$, etc. See figures 27, 28, 29.

Let $t$ start from $\breve{t} \in c$ and vary, reaching $t_{*}$ and penetrating into a neighbouring cell $c^{\prime}$ through $\left(\widetilde{H}_{a_{1} b_{1}} \cap \widetilde{H}_{a_{2} b_{2}} \cap \cdots \cap \widetilde{H}_{a_{l}, b_{l}}\right) \backslash \Delta$. At $t_{*}$ the above Stokes rays cross $R(\widetilde{\tau})$ clockwise and simultaneously, from the same side. Hence $Y_{\nu}^{\text {cont }}(z, t ; \widetilde{\tau}, c)$ has analytic continuation into $c^{\prime}$ (here the situation is similar to the continuation from $c_{3}$ to $c_{2}$ in figure 12). After the crossing, $t \in c^{\prime}$ and the same discussion of Part 1 applies. Namely, $Y_{\nu}^{\text {cont }}(z, t ; \widetilde{\tau}, c)=Y_{\nu}\left(z, t ; \widetilde{\tau}, c^{\prime}\right)$. The canonical asymptotics is extended up to the nearest Stokes ray in $S(\widetilde{\tau}, \widetilde{\tau}+\pi)$. Then, ${ }^{22}$ as in Proposition $13.3, Y_{\nu}\left(z, t ; \widetilde{\tau}, c^{\prime}\right)$ is analytically continued for $t$ back to $c$, up to $\breve{t}$. Therefore, the asymptotics of $Y_{\nu}^{\text {cont }}(z, t ; \widetilde{\tau}, c)$ gets extended up to the above mentioned nearest Stokes ray in $S(\widetilde{\tau}, \widetilde{\tau}+\pi)$. This fact holds also for $t=t_{*}$. In this way, $R_{a_{1} b_{1}}(t), R_{a_{2} b_{2}}(t)$, etc, get erased also at $t_{*}$. Proceeding as in Part 1 , we conclude that $Y_{\nu}\left(z, t_{*} ; \widetilde{\tau}\right) \equiv Y_{\nu}^{\text {cont }}\left(z, t_{*} ; \widetilde{\tau}, c\right)$ has asymptotics in the sector $\widehat{\mathcal{S}}_{\nu}\left(t_{*}\right)$.

Uniformity follows from Corollary 13.1 and Proposition 13.3 applied to any $Y_{\nu}\left(z, t ; \widetilde{\tau}, c^{\prime}\right)$.

Remark 14.4. If $\Lambda(0)=\lambda_{1} I$, then $\widehat{\mathcal{S}}_{\nu}=\mathcal{R}$, so that the asymptotics extends to $\mathcal{R}$.

- Proof of Theorem 14.1: We do the proof for $Y_{\nu+\mu}(z, t ; \widetilde{\tau}, c)$. For any other $Y_{\nu+k \mu}, k \in \mathbb{Z}$, the proof is the same. We compute the analytic continuation of $Y_{\nu+\mu}(z, t ; \widetilde{\tau}, c)$ along loops $\gamma_{a b}$ in $\pi_{1}\left(\mathcal{U}_{\epsilon_{0}}(0) \backslash \Delta, t_{\text {base }}\right)$, associated with $u_{a}(t)$ and $u_{b}(t)$ in (14.4). For these $a, b$, only one of the infinitely many rays of directions (14.6) is contained in $S(\widetilde{\tau}, \widetilde{\tau}+\pi)$ for $t \in c$. We can suppose that this is the ray

$$
R_{a b}(t):=\left\{z \in \mathcal{R} \mid \arg z=\frac{3 \pi}{2}-\arg _{p}\left(t_{a}-t_{b}\right)+2 N_{c} \pi\right\},
$$

$\left(\right.$ recall that $\left.\arg _{p}\left(u_{a}(t)-u_{b}(t)\right)=\arg _{p}\left(t_{a}-t_{b}\right)\right)$ where $N_{c}$ is a suitable integer such that

$$
\widetilde{\tau}<\frac{3 \pi}{2}-\arg _{p}\left(t_{a}-t_{b}\right)+2 N_{c} \pi<\widetilde{\tau}+\pi, \quad t \in c .
$$

If it is not the above ray, then it is a ray with $\arg z=\frac{3 \pi}{2}-\arg _{p}\left(t_{b}-t_{a}\right)+2 N_{c}^{\prime}$ and suitable $N_{c}^{\prime}$, so that the proof holds in the same way. $R_{a b}(t)$ rotates clockwise as $t$ moves along the support of $\gamma_{a b}$.

\footnotetext{
${ }^{22}$ By a small deformation $\widetilde{\tau} \mapsto \widetilde{\tau}+\varepsilon$.
} 


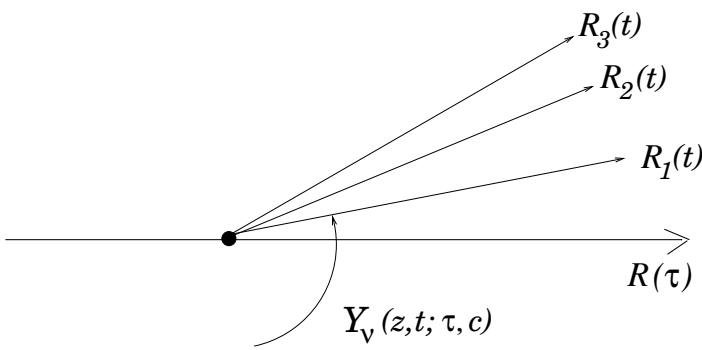

Figure 17. $Y_{\nu}(z, t ; \widetilde{\tau}, c)$ for $t \in c$, before $R_{1}(t)$ crosses $R(\widetilde{\tau})$. A portion of $\mathcal{S}_{\nu}(t)$ is represented by an arc.

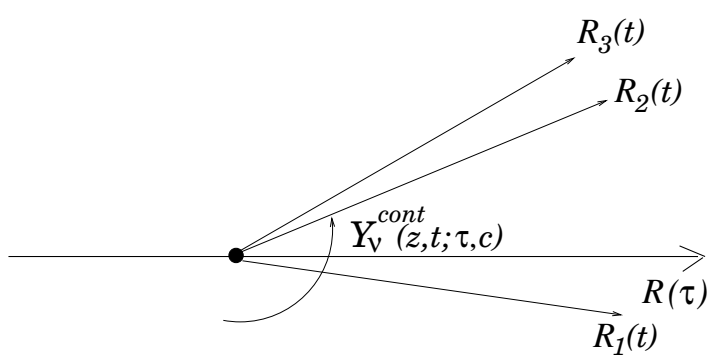

Figure 19. Extension of sector for the asymptotics of $Y_{\nu}^{\text {cont }}(z, t ; \widetilde{\tau}, c), t \in c_{1}$.

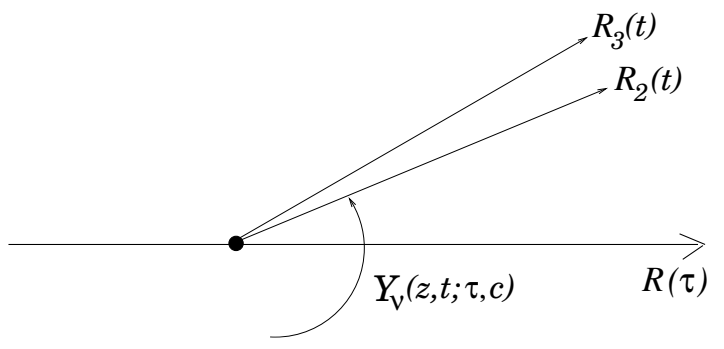

Figure 21. The sector where $Y_{\nu}(z, t ; \widetilde{\tau}, c)$ has canonical asymptotics has been extended up to $R_{2}(t), t \in c$.

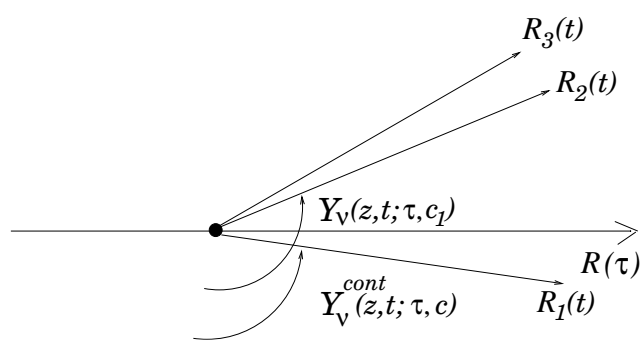

FIGURE 18. $Y_{\nu}^{\text {cont }}(z, t ; \widetilde{\tau}, c)$ and $Y_{\nu}\left(z, t ; \widetilde{\tau}, c_{1}\right)$ just after $R_{1}(t)$ has crossed $R(\widetilde{\tau})$. Portions of sectors where the asymptotics holds are represented.

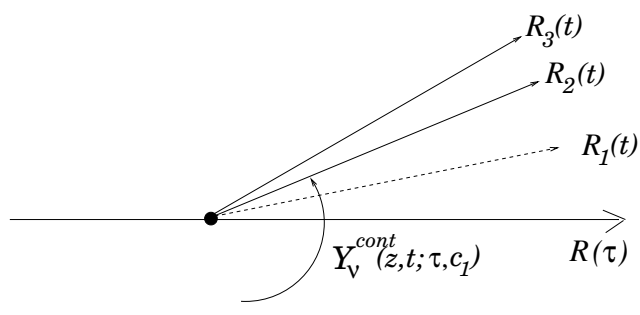

Figure 20. Continuation $Y_{\nu}^{\text {cont }}\left(z, t ; \widetilde{\tau}, c_{1}\right), t \in c$ before the crossing. The sector of the asymptotics is represented.

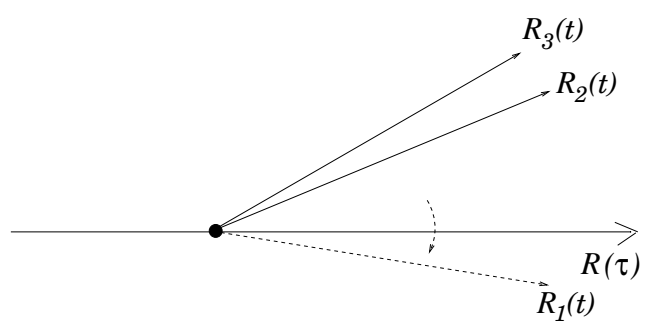

Figure 22. The dashed "virtual ray" $R_{1}(t)$ crosses $R(\widetilde{\tau})$, when $t$ enters into $c_{1}$.

For the sake of this proof, if a ray $R$ has angle $\theta$ and $R^{\prime}$ has angle $\theta+\theta^{\prime}$, we agree to write $R^{\prime}=R+\theta^{\prime}$. Hence, let

See Figure 31.

$$
R_{b a}(t):=R_{a b}(t)+\pi
$$

Assume first that $a, b$ are such that for $t \in c$ and $\left|t_{a}-t_{b}\right|$ sufficiently small, then no projected Stokes rays other than $P R_{a b}$ and $P R_{b a}$ cross $l(\widetilde{\tau})$ when $t$ varies along $\gamma_{a b}$ (the case discussed in figure 14). Cases when also other projected Stokes rays $\operatorname{cross} l(\widetilde{\tau})$, as for figure 15, will be discussed later.

Step 1) As base point consider $t_{0} \in c$, close to $\widetilde{H}_{a b}$, in such a way that $R_{a b}\left(t_{0}\right) \subset S(\widetilde{\tau}, \widetilde{\tau}+\pi)$ is close to $R(\widetilde{\tau}),{ }^{23}$ and it is the first ray in $S(\widetilde{\tau}, \widetilde{\tau}+\pi)$ encountered on moving anti-clockwise from $R(\widetilde{\tau})$.

$23 \widetilde{\tau}$ in $R(\widetilde{\tau})$ is the direction, while $t$ in $R_{a b}(t)$ is the dependence on $t$ 


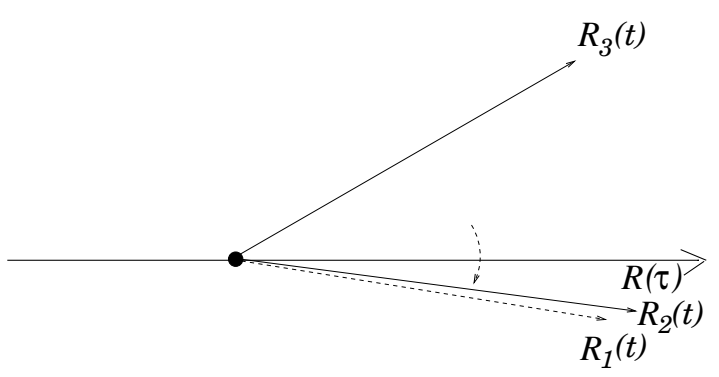

Figure 23. $R_{2}(t)$ crosses $R(\widetilde{\tau})$ when $t$ enters into $c_{2}$

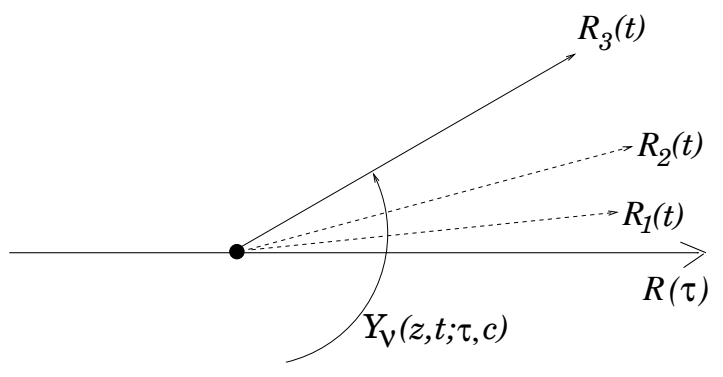

Figure 25. Extension up to $R_{3}(t)$ of the sector for the asymptotics of $Y_{\nu}(z, t ; \widetilde{\tau}, c)$, for $t \in c$.

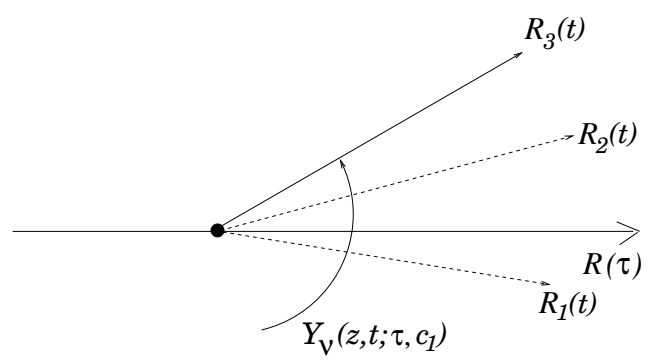

FiguRE 24. Extension up to $R_{3}(t)$ of the sector for the asymptotics of $Y_{\nu}\left(z, t ; \widetilde{\tau}, c_{1}\right)$, for $t \in c_{1}$.

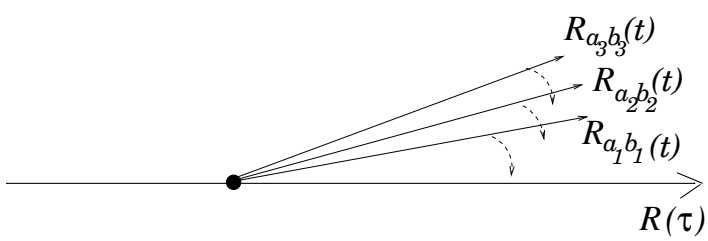

FIGURE 27. $t$ belongs to a cell $c$ whose boundary contains $\widetilde{H}_{a_{1} b_{1}} \cap \widetilde{H}_{a_{2} b_{2}} \cap \cdots \cap \widetilde{H}_{a_{l}, b_{l}}$, and such that the Stokes rays associated with these hyperplanes cross $R(\widetilde{\tau})$ simultaneously from the same side ( $c$ can be taken so that the crossing is clockwise).

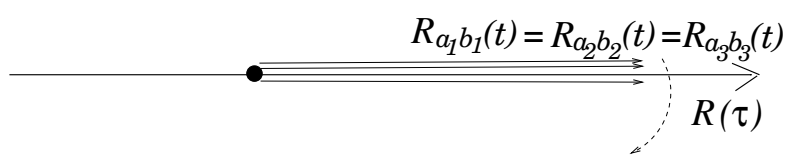

Figure 28. Simultaneous crossing for $t \in\left(\widetilde{H}_{a_{1} b_{1}} \cap \widetilde{H}_{a_{2} b_{2}} \cap \cdots \cap\right.$ $\left.\widetilde{H}_{a_{l}, b_{l}}\right) \backslash \Delta$.

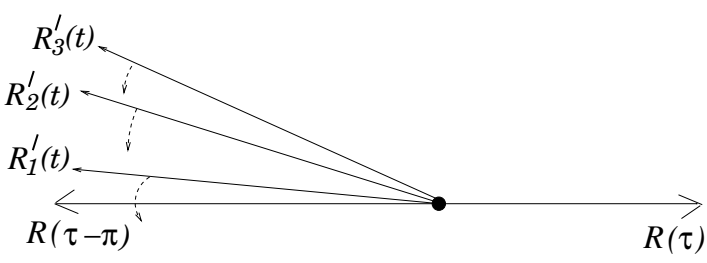

FiguRE 26. The extension of the sector for the asymptotics of $Y_{\nu}(z, t ; \widetilde{\tau}, c)$ must be done as above also at $R(\widetilde{\tau}-\pi)$, considering crossings as in figure.

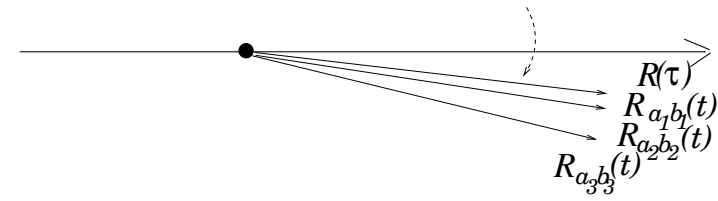

FigURE 29. After the simultaneous crossing, $t \in c^{\prime}$.

$Y_{\nu+\mu}\left(z, t_{0} ; \widetilde{\tau}, c\right)$ has the canonical asymptotics in $\mathcal{S}_{\nu+\mu}\left(t_{0}\right)$, which contains $R(\widetilde{\tau})$. By definition, $\mathcal{S}_{\nu+\mu}\left(t_{0}\right)$ contains $S(\widetilde{\tau}, \widetilde{\tau}+\pi)$ and extends to the closest Stokes rays outside. These rays are:

a) [left ray] the ray $R_{b a}\left(t_{0}\right)$.

b) [right ray] the first ray encountered on moving clockwise from $R_{a b}\left(t_{0}\right)$, which we call "the ray before" $R_{a b}\left(t_{0}\right)$ ( see Figure 31). The name "before" means that this ray comes before $R_{a b}\left(t_{0}\right)$ in the natural anti-clockwise orientation of angles). This ray is to the right of $R(\widetilde{\tau})$. 


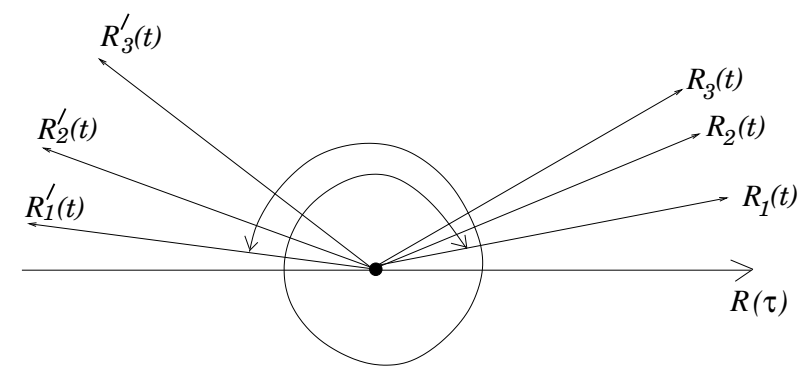

Figure 30. If $\Lambda(0)=\lambda_{1} I$, the asymptotics extends to $S\left(\arg \left(R_{1}(\breve{t})\right)-2 \pi, \arg \left(R_{1}^{\prime}(\breve{t})\right)+2 \pi\right)$.

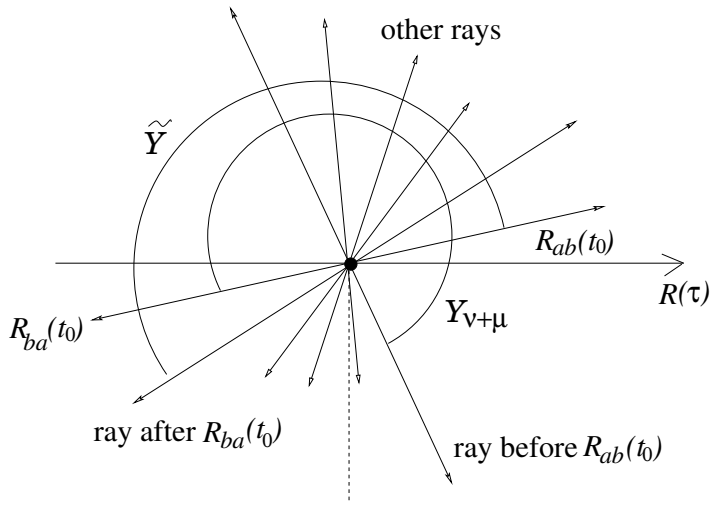

Figure 31 . This and the following pictures represent the sheet $S(\widetilde{\tau}-\pi / 2, \widetilde{\tau}+3 \pi / 2)$ (this is the meaning of the dashed vertical half-line). The Stokes rays at the starting point $t_{0}$ are represented. $Y_{\nu+\mu}$ is $Y_{\nu+\mu}(z, t ; \widetilde{\tau}, c)$, while $\widetilde{Y}$ is $Y_{\nu+\mu}\left(z, t ; \widetilde{\tau}, c^{\prime}\right)$

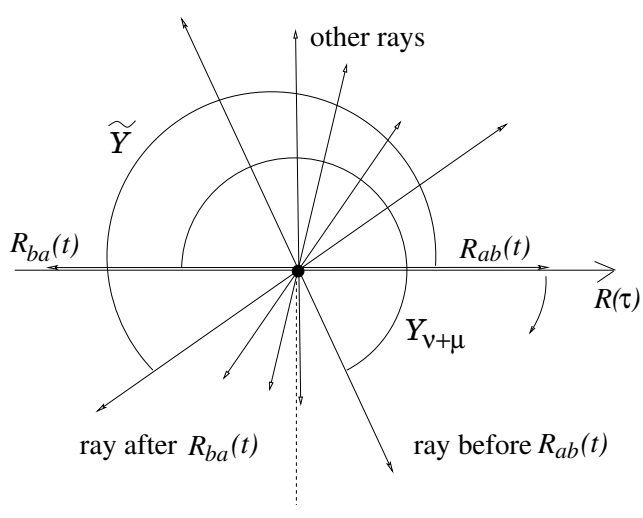

Figure 32. Crossing of $R(\widetilde{\tau})$. Note that also the other rays can move, but never cross the admissible ray $R(\widetilde{\tau})$ or $R(\widetilde{\tau} \pm$ $\pi)$.

Step 2) As $t$ moves along $\gamma_{a b}, R_{a b}(t)$ moves clockwise and crosses $R(\widetilde{\tau})$, while $R_{b a}(t)$ crosses $R(\widetilde{\tau}+\pi)$ (see Figure 32). The curve $\gamma_{a b}$ crosses $\widetilde{H}_{a b} \backslash \Delta$ and penetrates into another cell $c^{\prime}$. As in Proposition 13.3, just before the intersection of the curve with $\widetilde{H}_{a b} \backslash \Delta$, also $Y_{\nu+\mu}\left(z, t ; \widetilde{\tau}, c^{\prime}\right)$ is well defined with the same asymptotics as $Y_{\nu+\mu}(z, t ; \widetilde{\tau}, c)$, but in the sector bounded by $R_{a b}(t)$, as right ray, and the ray coming after $R_{b a}(t)$ in anti-clockwise sense, as left ray, which we call "the ray after" (see Figures 31 and 32). A connection matrix $\mathbb{K}^{[a b]}(t)$ (called Stokes factor) connects $Y_{\nu+\mu}\left(z, t ; \widetilde{\tau}, c^{\prime}\right)$ and $Y_{\nu+\mu}(z, t ; \widetilde{\tau}, c)$,

$$
Y_{\nu+\mu}\left(z, t ; \widetilde{\tau}, c^{\prime}\right)=Y_{\nu+\mu}(z, t ; \widetilde{\tau}, c) \mathbb{K}^{[a b]}(t) .
$$

$\mathbb{K}^{[a b]}(t)$ is holomorphic on $\mathcal{U}_{\epsilon_{0}}(0)$, because the fundamental solutions are holomorphic by assumption 2$)$. Again by the proof of Proposition 13.3, just after the crossing, $Y_{\nu+\mu}(z, t ; \widetilde{\tau}, c)$ maintains its asymptotics between the ray before $R_{a b}(t)$, which has possibly only slightly moved, and $R_{b a}(t)$. Both $Y_{\nu+\mu}(z, t ; \widetilde{\tau}, c)$ and $Y_{\nu+\mu}\left(z, t ; \widetilde{\tau}, c^{\prime}\right)$ have the same asymptotics in successive sectors, and in particular they have the same asymptotics on the sector having right ray $R_{a b}$ and left ray $R_{b a}$. Since $\Re\left[\left(u_{a}-u_{b}\right) z\right]>0$ on this sector, it follows from (14.8) that for $t$ in a small open neighbourhood of the intersection point of the curve with $\widetilde{H}_{a b} \backslash \Delta$, the structure of $\mathbb{K}^{[a b]}(t)$ must be as follows

$$
\left(\mathbb{K}^{[a b]}\right)_{i i}=1, \quad 1 \leq i \leq n ; \quad\left(\mathbb{K}^{[a b]}\right)_{i j}=0 \quad \forall \quad i \neq j \text { except for } i=b, j=a .
$$

The entry $\left(\mathbb{K}^{[a b]}\right)_{b a}(t)$ may possibly be different from zero. Since $\mathbb{K}^{[a b]}(t)$ is holomorphic on $\mathcal{U}_{\epsilon_{0}}(0)$, the above structure holds for every $t \in \mathcal{U}_{\epsilon_{0}}(0)$. 


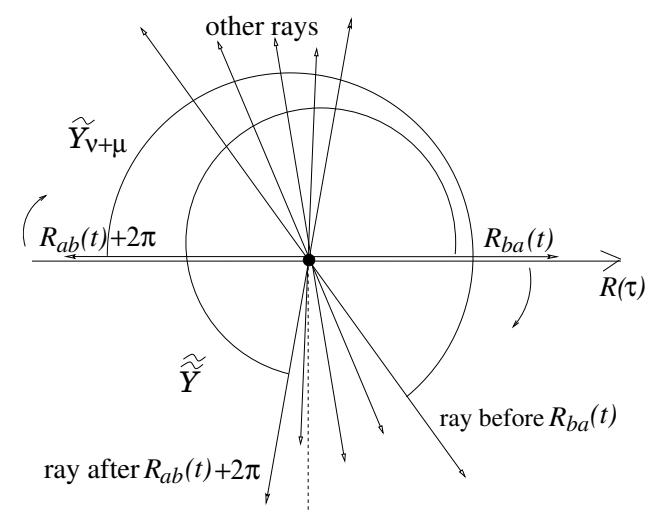

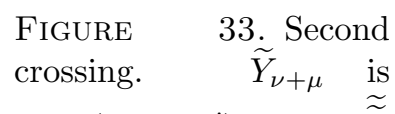
$Y_{\nu+\mu}\left(z, t ; \widetilde{\tau}, c^{\prime}\right)$ and $\widetilde{\widetilde{Y}}$ is $Y_{\nu+\mu}(z, t ; \widetilde{\tau}, c)$. The other rays represented are moving, without

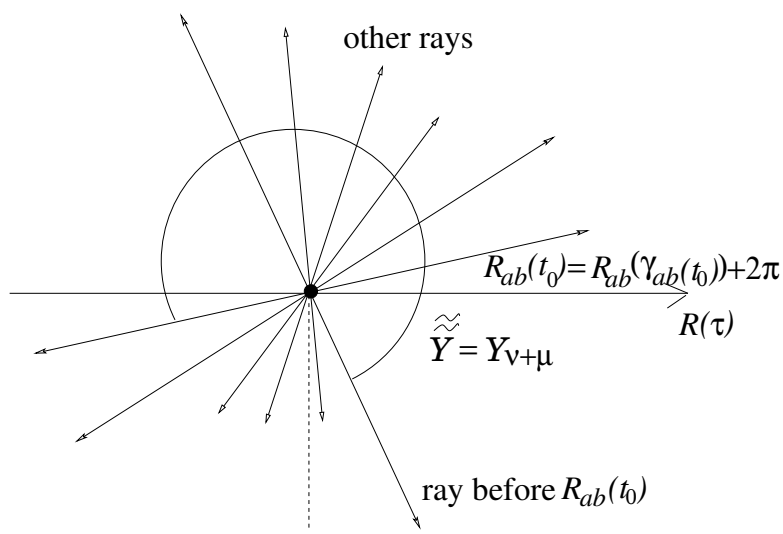

Figure 34. After the loop $\gamma_{a b}$ crossing $R(\widetilde{\tau})$ or $R(\widetilde{\tau} \pm$ $\pi)$.

Step 3) As $t$ moves along $\gamma_{a b}, R_{a b}(t)$ continues to rotate clockwise. It will cross other Stokes rays along the way, but $Y_{\nu+\mu}\left(z, t ; \widetilde{\tau}, c^{\prime}\right)$ will maintain its canonical asymptotics in $\mathcal{S}_{\nu+\mu}(t)$, because $t \in c^{\prime}$, until $R_{a b}(t)$ reaches $R(\widetilde{\tau}-\pi)$.

Step 4) Just before $R_{a b}(t)$ crosses $R(\widetilde{\tau}-\pi), \mathcal{S}_{\nu+\mu}(t)$ has left ray equal to $R_{a b}(t)+2 \pi$ and the right ray is the ray before $R_{b a}(t)$. Again by Proposition $13.3, Y_{\nu+\mu}(z, t ; \widetilde{\tau}, c)$ is defined with canonical asymptotics in the sector following $\mathcal{S}_{\nu+\mu}(t)$ anticlockwise (see Figure 33). There is a Stokes factor $\widetilde{\mathbb{K}}^{[a b]}(t)$ such that,

$$
Y_{\nu+\mu}(z, t ; \widetilde{\tau}, c)=Y_{\nu+\mu}\left(z, t ; \widetilde{\tau}, c^{\prime}\right) \widetilde{\mathbb{K}}^{[a b]}(t) .
$$

The above relation and the common asymptotic behaviour imply that for $t$ in a neighbourhood of the crossing point the structure must be

$$
\left(\widetilde{\mathbb{K}}^{[a b]}\right)_{i i}=1, \quad 1 \leq i \leq n ; \quad\left(\widetilde{\mathbb{K}}^{[a b]}\right)_{i j}=0 \quad \forall \quad i \neq j \text { except for } i=a, j=b .
$$

The entry $(\widetilde{\mathbb{K}})_{a b}^{[a b]}(t)$ may be possibly non zero. By assumption 2$), \widetilde{\mathbb{K}}^{[a b]}(t)$ is holomoprhic on $\mathcal{U}_{\epsilon_{0}}(0)$, so the above structure holds for any $t \in \mathcal{U}_{\epsilon_{0}}(0)$.

Step 5) The rotation of $R_{a b}(t)$ continues, crossing other Stokes rays. Finally, $R_{a b}(t)$ reaches the position

$$
R_{a b}\left(\gamma_{a b}\left(t_{0}\right)\right)=R_{a b}\left(t_{0}\right)-2 \pi
$$

after a full rotation of $-2 \pi$. This corresponds to the full loop $t_{a}-t_{b} \mapsto\left(t_{a}-t_{b}\right) e^{2 \pi i}$.

From (14.8) and (14.9) we conclude that,

$$
Y_{\nu+\mu}(z, t ; \widetilde{\tau}, c)=Y_{\nu+\mu}(z, t ; \widetilde{\tau}, c) \mathbb{K}^{[a b]}(t) \widetilde{\mathbb{K}}^{[a b]}(t), \quad t \in \mathcal{U}_{\epsilon_{0}}(0) .
$$

Hence

$$
\mathbb{K}^{[a b]}(t) \widetilde{\mathbb{K}}^{[a b]}(t)=I, \quad t \in \mathcal{U}_{\epsilon_{0}}(0) .
$$

This implies that $\left(\mathbb{K}^{[a b]}\right)_{b a}=\left(\widetilde{\mathbb{K}}^{[a b]}\right)_{a b}=0$. Therefore,

$$
\mathbb{K}^{[a b]}(t)=\widetilde{\mathbb{K}}^{[a b]}(t)=I, \quad t \in \mathcal{U}_{\epsilon_{0}}(0) .
$$

We conclude from (14.8) or (14.9) that

$$
Y_{\nu+\mu}(z, t ; \widetilde{\tau}, c)=Y_{\nu+\mu}\left(z, t ; \widetilde{\tau}, c^{\prime}\right), \quad t \in \mathcal{U}_{\epsilon_{0}}(0) .
$$

The above discussion can be repeated for all loops $\gamma_{a b}$ starting in $c$ involving a simple crossing of $R(\widetilde{\tau})$. 
We now turn to the case when also other projected Stokes rays, not only $P R_{a b}$ and $P R_{b a}$, cross $l(\widetilde{\tau})$ along $\gamma_{a b}$. In this case, the representative of $\gamma_{a b}$ can be decomposed into steps, for each of which the analytic continuation studied above and formula (14.12) hold. See for example the configuration of figure 15. In these occurrences, the analytic continuation is done first from $c$ to $c^{\prime}$. The passage from $c$ to $c^{\prime}$ corresponds to the alignment of $u_{\gamma}$ and $u_{a}$. Hence, $Y_{\nu+\mu}(z, t ; \widetilde{\tau}, c)$ is continued from $c$ to $c^{\prime}$ and (14.12) holds. Then, $Y_{\nu+\mu}\left(z, t ; \widetilde{\tau}, c^{\prime}\right)$ can be used in place of $Y_{\nu+\mu}(z, t ; \widetilde{\tau}, c)$, applying the same proof previously explained, since for $t \in c^{\prime}$, if $\left|t_{a}-t_{b}\right|$ is sufficiently small, then the crossing involves only $P R_{a b}$ and $P R_{b a}$.

Concluding, (14.12) holds for any cell $c^{\prime}$ which has a boundary in common with $c$.

Now, we consider a cell $c^{\prime}$ which has a boundary in common with $c$, and we do the analytic continuation of $Y_{\nu+\mu}\left(z, t ; \widetilde{\tau}, c^{\prime}\right)$ to all cells $c^{\prime \prime}$ which have a boundary in common with $c^{\prime}$, in the same way it was done above. In this way, we conclude that $Y_{\nu+\mu}(z, t ; \widetilde{\tau}, c)=Y_{\nu+\mu}\left(z, t ; \widetilde{\tau}, c^{\prime}\right)$ and $Y_{\nu+\mu}\left(z, t ; \widetilde{\tau}, c^{\prime}\right)=$ $Y_{\nu+\mu}\left(z, t ; \widetilde{\tau}, c^{\prime \prime}\right)$, for $t \in \mathcal{U}_{\epsilon_{0}}(0)$. With this procedures, all cells can be reached, so that (14.12) holds for any cell $c$ and $c^{\prime}$ of $\mathcal{U}_{\epsilon_{0}}(0)$. For the above reasons, we are allowed to write

$$
Y_{\nu+\mu}(z, t ; \widetilde{\tau}), \quad t \in \mathcal{U}_{\epsilon_{0}}(0)
$$

in place of $Y_{\nu+\mu}(z, t ; \widetilde{\tau}, c)$.

The above conclusions imply that the assumptions of Lemma 14.1 hold. Lemma 14.1 assures that the asymptotics extends to the closest Stokes rays in $\mathfrak{R}(t)$ outside $S(\widetilde{\tau}, \widetilde{\tau}+\pi)$. Hence the asymptotics

$$
G_{0}(t)^{-1} Y_{\nu+\mu}(z, t ; \widetilde{\tau}) e^{-\Lambda(t)} z^{-B_{1}(t)} \sim I+\sum_{k=0}^{\infty} F_{k}(t) z^{-k}
$$

holds for $z \rightarrow \infty$ in $\widehat{\mathcal{S}}_{\nu+\mu}(t)$, and $t \in \mathcal{U}_{\epsilon_{0}}(0) \backslash \Delta$. A fortiori, the asymptotics holds in $\widehat{\mathcal{S}}_{\nu+\mu}=\widehat{\mathcal{S}}_{\nu+\mu}\left(\mathcal{U}_{\epsilon_{0}}(0)\right)$. It is uniform on any compact subset $K \subset \mathcal{U}_{\epsilon_{0}}(0) \backslash \Delta$ for $z \rightarrow \infty$ in $\widehat{\mathcal{S}}_{\nu}(K)$.

The last property to be verified is that the asymptotics in $\widehat{\mathcal{S}}_{\nu+\mu}$ holds also for $t \in \Delta$. Let

$$
R_{k}(z, t):=G_{0}(t)^{-1} Y_{\nu+\mu}(z, t ; \widetilde{\tau}) e^{-\Lambda(t) z} z^{-B_{1}(t)}-\left(I+\sum_{l=1}^{k-1} F_{l}(t) z^{-k}\right), \quad t \in \mathcal{U}_{\epsilon_{0}}(0) .
$$

Let $\left(R_{k}(z, t)\right)_{l s}, l, s=1, \ldots, n$ be the entries of the matrix $R_{k}$. Since $R_{k}$ is the $k$-th remainder of the asymptotic expansion, it satisfies the inequality

$$
\left|R_{k}(z, t)\right|:=\max _{l, s=1, \ldots, n}\left|\left(R_{k}(z, t)\right)_{l s}\right| \leq \frac{C(k ; \bar{S} ; t)}{|z|^{k}}, \quad t \in \mathcal{U}_{\epsilon_{0}}(0) \backslash \Delta, \quad z \in \bar{S},
$$

for $z$ belonging to a proper closed subsector $\bar{S} \subset \widehat{\mathcal{S}}_{\nu+\mu}$. Here $C(k ; \bar{S} ; t)$ is a constant depending on $k, \bar{S}$ and $t \in \mathcal{U}_{\epsilon_{0}}(0) \backslash \Delta$. Our goal is to prove a similar relation for $t \in \Delta$.

We consider $n$ positive numbers $r_{a} \leq \epsilon_{0}, a=1, \ldots, n$. We further require that for any $i=1, \ldots, s$ and for any $a \neq b$, such that $u_{a}(0)=u_{b}(0)=\lambda_{i}$, these numbers are distinct, i.e. $r_{a} \neq r_{b}$. We introduce the polydisc $\mathcal{U}_{r_{1}, \ldots, r_{n}}(0):=\left\{t \in \mathbb{C}^{n}|| t_{a} \mid \leq r_{a}, a=1, \ldots, n\right\}$. Clearly, $\mathcal{U}_{r_{1}, \ldots, r_{n}}(0) \subset \mathcal{U}_{\epsilon_{0}}(0)$. Let us denote the skeleton of $\mathcal{U}_{r_{1}, \ldots, r_{n}}(0)$ with $\Gamma:=\left\{t \in \mathbb{C}^{n}|| t_{a} \mid=r_{a}, a=1, \ldots, n\right\}$. The above choice of pairwise distinct $r_{a}$ 's assures that $\Gamma \cap \Delta=\emptyset$.

The inequality (14.15) holds in $\mathcal{U}_{r_{1}, \ldots, r_{n}}(0) \backslash \Delta$ for any fixed $z \in \bar{S}$. Since $R_{k}(z, t)$ is holomorphic on the interior of $\mathcal{U}_{r_{1}, \ldots, r_{n}}(0)$ and continuous up to the boundary, every matrix entry of $R_{k}(z, t)$ attains its maximum modulus on the Shilov boundary (cf. [60], page 21-22) of $\mathcal{U}_{r_{1}, \ldots, r_{n}}(0)$, which coincides with $\Gamma$. Since (14.15) holds on $\Gamma$, we conclude that

$$
\left|R_{k}(z, t)\right| \leq \frac{C(k ; \bar{S} ; \Gamma)}{|z|^{k}}, \quad \forall t \in \mathcal{U}_{r_{1}, \ldots, r_{n}}(0),
$$

where $C(k ; \bar{S} ; \Gamma)=\max _{t \in \Gamma} C(k ; \bar{S} ; t)$. This maximum is finite, because the asymptotics is uniform on every compact subset of $\mathcal{U}_{\epsilon_{0}}(0) \backslash \Delta$. The above estimate (14.16) means that the asymptotics (14.14) holds uniformly in $t$ on the whole $\mathcal{U}_{r_{1}, \ldots, r_{n}}(0)$, including $\Delta$, for $z \rightarrow \infty$ in $\bar{S}$. A fortiori, the asymptotics holds in $\mathcal{U}_{\epsilon_{1}}(0)$, with $\epsilon_{1} \leq \min _{a} r_{a}<\epsilon_{0}$. Since (14.16) holds for any closed proper subsector $\bar{S} \subset \widehat{\mathcal{S}}_{\nu+\mu}$, by definition $G_{0}(t)^{-1} Y_{\nu+\mu}(z, t ; \widetilde{\tau}) e^{-\Lambda(t)} z^{-B_{1}(t)}$ is asymptotic to $I+\sum_{k=0}^{\infty} F_{k}(t) z^{-k}$ in $\widehat{\mathcal{S}}_{\nu+\mu}$. 
It remains to comment on the structure of a Stokes matrix. In the proof above, a ray $R_{a b}(t)$ associated with a pair $u_{a}(t), u_{b}(t)$ with $u_{a}(0)=u_{b}(0)=\lambda_{i}$ is "invisible" as far as the asymptotics is concerned, because $\mathbb{K}^{[a b]}(t)=\widetilde{\mathbb{K}}^{[a b]}(t)=I$ for any $\gamma_{a b}$. Therefore, in the factorisation of any $\mathbb{S}_{\nu}(t)$, the Stokes factors associated with rays $3 \pi / 2-\arg \left(u_{a}(t)-u_{b}(t)\right) \bmod 2 \pi$, with $u_{a}(0)=u_{b}(0)=\lambda_{i}$, are the identity.

\section{Meromorphic Continuation}

In Theorem 14.1 we have assumed that for any $\widetilde{\tau}$-cell $c$ of $\mathcal{U}_{\epsilon_{0}}(0)$ and any $k \in \mathbb{Z}$, the fundamental solution $Y_{\nu+k \mu}(z, t ; \widetilde{\tau}, c)$ has analytic continuation as a single-valued holomorphic function on the whole $\mathcal{U}_{\epsilon_{0}}(0)$. In this section, we assume that the above fundamental matrices have continuation on the universal covering $\mathcal{R}\left(\mathcal{U}_{\epsilon_{0}}(0) \backslash \Delta\right)$ of $\mathcal{U}_{\epsilon_{0}}(0) \backslash \Delta$ as meromorphic matrix-valued functions. We show that if the Stokes matrices satisfy a vanishing condition, then the continuation is actually holomorphic and single valued on $\mathcal{U}_{\epsilon_{0}}(0) \backslash \Delta$. In particular, $\Delta$ is not a branching locus.

Recall that the Stokes matrices are defined by

$$
Y_{\nu+(k+1) \mu}(z, t ; \widetilde{\tau}, c)=Y_{\nu+k \mu}(z, t ; \widetilde{\tau}, c) \mathbb{S}_{\nu+k \mu}(t), \quad \text { for } t \in c .
$$

Theorem 15.1. Consider the system (2.1) (i.e. system (1.1) of the Introduction) with holomorphic coefficients and Assumption 1. Let $\Lambda(t)$ be of the form (1.2), with eigenvalues (1.15) and $\epsilon_{0}=\delta_{0}$ as in subsection 14.1. Let $\widetilde{\tau}$ be the direction of an admissible ray $R(\widetilde{\tau})$, satisfying $\tau_{\nu}<\widetilde{\tau}<\tau_{\nu+1}$.

Assume that for any $\widetilde{\tau}$-cell $c$ of $\mathcal{U}_{\epsilon_{0}}(0)$ and any $k \in \mathbb{Z}$, the fundamental solution $Y_{\nu+k \mu}(z, t ; \widetilde{\tau}, c)$, defined for $t \in c$, has analytic continuation on the universal covering $\mathcal{R}\left(\mathcal{U}_{\epsilon_{0}}(0) \backslash \Delta\right)$ as a meromorphic matrix-valued function. Assume that the entries of the Stokes matrices satisfy the vanishing condition

$$
\left(\mathbb{S}_{\nu}(t)\right)_{a b}=\left(\mathbb{S}_{\nu}(t)\right)_{b a}=\left(\mathbb{S}_{\nu+\mu}(t)\right)_{a b}=\left(\mathbb{S}_{\nu+\mu}(t)\right)_{b a}=0, \quad \forall t \in c,
$$

for any $1 \leq a \neq b \leq n$ such that $u_{a}(0)=u_{b}(0)$.

Then:

- The continuation of $Y_{\nu+k \mu}(z, t ; \widetilde{\tau}, c)$ defines a single-valued holomorphic (matrix-valued) function on $\mathcal{U}_{\epsilon_{0}}(0) \backslash \Delta$.

- $Y_{\nu+k \mu}(z, t ; \widetilde{\tau}, c)=Y_{\nu+k \mu}\left(z, t ; \widetilde{\tau}, c^{\prime}\right)$, for $t \in c$. Therefore, we write $Y_{\nu+k \mu}(z, t ; \widetilde{\tau})$

- The asymptotics

$$
G_{0}^{-1}(t) Y_{\nu+k \mu}(z, t ; \widetilde{\tau}) e^{-\Lambda(t) z} z^{-B_{1}(t)} \sim I+\sum_{j \geq 1} F_{j}(t) z^{-j},
$$

holds for $z \rightarrow \infty$ in $\widehat{\mathcal{S}}_{\nu+k \mu}(t), t \in \mathcal{U}_{\epsilon_{0}}(0) \backslash \Delta$.

Remark 15.1. Recall that $B_{1}(t)=\operatorname{diag}\left(\widehat{A}_{1}(t)\right)$ is the exponent of formal monodromy, appearing in the fundamental solutions (12.1). The formula $\mathbb{S}_{\nu+2 \mu}=e^{-2 \pi i B_{1}} \mathbb{S}_{\nu} e^{2 \pi i B_{1}}$, analogous to that of Proposition 6.2 , implies that (15.1) holds for any $\mathbb{S}_{\nu+k \mu}$. Notice that the $F_{j}(t)$ 's are holomorphic on $\mathcal{U}_{\epsilon_{0}}(0) \backslash \Delta$.

Proof: Without loss of generality, we label the eigenvalues as in (5.1)-(5.4), so that $\mathbb{S}_{\nu+k \mu}(t)$ is partitioned into $p_{j} \times p_{k}$ blocks $(1 \leq j, k \leq s)$ such that the $p_{j} \times p_{j}$ diagonal blocks have matrix entries $\left(\mathbb{S}_{\nu+k \mu}(t)\right)_{a b}$ corresponding to coalescing eignevalues $u_{a}(0)=u_{b}(0)$.

We consider $Y_{\nu+\mu}(z, t ; \tilde{\tau}, c)$. For any other $Y_{\nu+k \mu}(z, t ; \tilde{\tau}, c)$ the discussion is analogous. We denote the meromorphic continuation of $Y_{\nu+\mu}(z, t ; \widetilde{\tau}, c)$ on $\mathcal{R}\left(\mathcal{U}_{\epsilon_{0}}(0) \backslash \Delta\right)$ by $\mathbb{Y}_{\nu+\mu}(z, \tilde{t} ; \tilde{\tau}, c), \tilde{t} \in \mathcal{R}\left(\mathcal{U}_{\epsilon_{0}}(0) \backslash \Delta\right)$. Therefore, the continuation along a loop $\gamma_{a b}$ as in (14.4) and (14.5), starting in $c$, will be denoted by $\mathbb{Y}_{\nu+\mu}\left(z, \gamma_{a b} t ; \tilde{\tau}, c\right)$, where $\tilde{t}=\gamma_{a b} t$ is the point in $\mathcal{R}\left(\mathcal{U}_{\epsilon_{0}}(0) \backslash \Delta\right)$ after the loop.

We then proceed as in the proof of Theorem 14.1, up to eq. (14.10). Assume first that $a, b$ are such that for $t \in c$ and $\left|t_{a}-t_{b}\right|$ sufficiently small, then no projected Stokes rays other than $P R_{a b}$ and $P R_{b a}$ cross $l(\widetilde{\tau})$ when $t$ varies along $\gamma_{a b}$ (the case discussed in figure 14). Cases when also other projected Stokes rays $\operatorname{cross} l(\widetilde{\tau})$, as for figure 15, can be discussed later as we did in the proof of Theorem 14.1. 
The intermediate steps along $\gamma_{a b}$, corresponding to the formulae (14.8) and (14.9), hold. Namely:

$$
Y_{\nu+\mu}\left(z, t ; \widetilde{\tau}, c^{\prime}\right)=Y_{\nu+\mu}(z, t ; \widetilde{\tau}, c) \mathbb{K}^{[a b]}(t)
$$

for $t$ in a neighbourhood of the intersection of the support of $\gamma_{a b}$ with the common boundary of $c$ and $c^{\prime}$ (i.e. $\left.\widetilde{H}_{a b} \backslash \Delta\right)$ corresponding to $R_{a b}$ crossing $R(\widetilde{\tau})$. Moreover,

$$
Y_{\nu+\mu}(z, t ; \tilde{\tau}, c)=Y_{\nu+\mu}\left(z, t ; \widetilde{\tau}, c^{\prime}\right) \widetilde{\mathbb{K}}^{[a b]}(t),
$$

for $t$ in a neighbourhood of the intersection of the support of $\gamma_{a b}$ with the common boundary of $c$ and $c^{\prime}$ corresponding to $R_{a b}$ crossing $R(\widetilde{\tau}-\pi)$. Note that to such $t$ there corresponds a point $\tilde{t}$ in the covering, which is reached along $\gamma_{a b}$, so that $Y_{\nu+\mu}(z, t ; \widetilde{\tau}, c)$ in the right hand-side of $(15.2)$ becomes $\mathbb{Y}_{\nu+\mu}(z, \tilde{t} ; \tilde{\tau}, c)$.

$\mathbb{K}^{[a b]}(t), \widetilde{\mathbb{K}}^{[a b]}(t)$ have the same structure as in the proof of Theorem 14.1, for $t$ in a small open neighborhood of the crossing points. By assumption, $\mathbb{K}^{[a b]}(t), \widetilde{\mathbb{K}}^{[a b]}(t)$ are meromorphic on $\mathcal{R}\left(\mathcal{U}_{\epsilon_{0}}(0) \backslash \Delta\right)$, so they preserve their structure.

At the end of the loop, $t$ is back to the initial point, but in the universal covering the point $\tilde{t}=\gamma_{a b} t$ is reached and $Y_{\nu+\mu}(z, t ; \widetilde{\tau}, c)$ has been analytically continued to $\mathbb{Y}_{\nu+\mu}\left(z, \gamma_{a b} t ; \tilde{\tau}, c\right)$. Thus, the analogous of formula (14.10) now reads as follows

$$
Y_{\nu+\mu}(z, t ; \widetilde{\tau}, c)=\mathbb{Y}_{\nu+\mu}\left(z, \gamma_{a b} t ; \widetilde{\tau}, c\right) \mathbb{K}^{[a b]}(t) \widetilde{\mathbb{K}}^{[a b]}(t), \quad t \in c .
$$

We need to compute the only non trivial entries $\left(\mathbb{K}^{[a b]}(t)\right)_{b a}$ and $\left(\widetilde{\mathbb{K}}^{[a b]}(t)\right)_{a b}$. Let us consider $\mathbb{K}^{[a b]}(t)$. As it is well known, $\mathbb{S}_{\nu+\mu}$ can be factorised into Stokes factors. At the beginning of the loop $\gamma_{a b}$, just before $t$ crosses the boundary of the cell $c$ as in Figure 31, we have

$$
\mathbb{S}_{\nu+\mu}=\mathbb{K}^{[a b]} \cdot \mathbb{T}
$$

where $\mathbb{K}^{[a b]}$ is a Stokes factor and the matrix $\mathbb{T}$ is factorised into the remaining Stokes factors of $\mathbb{S}_{\nu+\mu}$. For simplicity, we suppose that $\mathbb{S}_{\nu+\mu}$ is upper triangular (namely $a<b$; if not, the discussion is modified in an obvious way):

$$
\mathbb{S}_{\nu+\mu}=\left(\begin{array}{ccccc}
I_{p_{1}} & * & * & \cdots & * \\
0 & I_{p_{2}} & * & \cdots & * \\
0 & 0 & I_{p_{3}} & \cdots & * \\
\vdots & \vdots & \vdots & \ddots & \vdots \\
0 & 0 & 0 & 0 & I_{p_{s}}
\end{array}\right)
$$

It follows that $b<a$, namely $\mathbb{K}^{[a b]}$ has entries equal to 1 's on the diagonal, 0 elsewhere, except for a non-trivial entry $m_{b a}:=\left(\mathbb{K}^{[a b]}\right)_{b a}$ above the diagonal in a block corresponding to one of the $I_{p_{1}}, \ldots, I_{p_{s}}$ in (15.5). Let $E_{j k}$ be the matrix with zero entries except for $\left(E_{j k}\right)_{j k}=1$. Then, $\mathbb{K}^{[a b]}=I+m_{b a} E_{b a}$, and we factorise $\mathbb{T}$ as follows:

$$
\mathbb{S}_{\nu+\mu}=\left(I+m_{b a} E_{b a}\right) \cdot \prod_{j<k \text { in } V}\left(I+m_{j k} E_{j k}\right) \cdot \prod_{\text {The others } j<k}\left(I+m_{j k} E_{j k}\right),
$$

where $V$ is the set of indices $j<k \in\{1,2, \ldots, n\}$ such that $u_{j}(0)=u_{k}(0)$ and $(j, k) \neq(b, a)$ (the entries of the diagonal blocks of the matrix block partition associated with $\left.p_{1}, \ldots, p_{s}\right)$.

Now, all the numbers $m_{b a}$ and $m_{j k}$ are uniquely determined by the entries of $\mathbb{S}_{\nu+\mu}$. This fact follows from the following result (see for example [2]). Let $S$ be any upper triangular matrix with diagonal elements equal to 1 . Label the upper triangular entries entries $(j, k), j<k$, in an arbitrary way,

$$
\left(j_{1}, k_{1}\right), \quad\left(j_{2}, k_{2}\right), \quad \ldots, \quad\left(j_{\frac{n(n-1)}{2}}, k_{\frac{n(n-1)}{2}}\right) .
$$

Then, there exists numbers $m_{1}, m_{2}, \ldots, m_{\frac{n(n-1)}{2}}$ which are uniquely determined by the labelling and the entries of $S$, such that

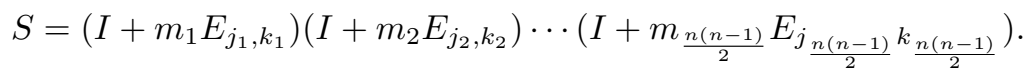

Indeed, a direct computation gives

$$
S=I+\sum_{a=1}^{\frac{n(n-1)}{2}} m_{a} E_{j_{a} k_{a}}+\text { non linear terms in the } m_{a} \text { 's. }
$$


The commutation relations

$$
E_{i j} E_{j k}=E_{i k}, \quad E_{i j} E_{l k}=0 \text { for } j \neq l,
$$

imply that the non linear terms are in an upper sub-diagonal lying above the sub-diagonal where the corresponding factors appear. Hence, (15.6) gives uniquely solvable recursive relations, expressing the $m_{a}$ 's in terms of the entries of $S$.

Applying the above procedure to $S=\mathbb{S}_{\nu+\mu}$, and keeping (15.1) into account, we obtain

$$
m_{b a}=0, \quad m_{j k}=0 \forall j<k \text { in } V .
$$

This proves that

$$
\mathbb{K}^{[a b]}(t)=I
$$

for $t$ in a small open neighborhood of the intersection point of the curve $\gamma_{a b}$ with $\widetilde{H}_{a b} \backslash \Delta$. This structure is preserved by analytic continuation. Analogously, we factorise into Stokes factor the (lower triangular) matrix $\mathbb{S}_{\nu}=\widetilde{\mathbb{T}} \cdot \widetilde{\mathbb{K}}^{[a b]}$ and prove that

$$
\widetilde{\mathbb{K}}^{[a b]}=I .
$$

We conclude that

$$
Y_{\nu+\mu}(z, t ; \widetilde{\tau}, c)=\mathbb{Y}_{\nu+\mu}\left(z, \gamma_{a b} t ; \widetilde{\tau}, c\right) .
$$

Formulae (15.2) and (15.3) also imply that

$$
Y_{\nu+\mu}\left(z, t ; \widetilde{\tau}, c^{\prime}\right)=Y_{\nu+\mu}(z, t ; \widetilde{\tau}, c)
$$

This discussion can be repeated for any loop and any cell, as we did in the proof of Theorem 14.1 in the paragraphs following eq. (14.12). Since $Y_{\nu+\mu}(z, t ; \tilde{\tau}, c)$ is holomorphic on $c$ by Corollary 13.1, the above formulae imply the analyticity of $Y_{\nu+\mu}\left(z, t ; \widetilde{\tau}, c^{\prime}\right)$ on $\mathcal{U}_{\epsilon_{0}}(0) \backslash \Delta$. Since (15.7) holds, the first two statements are proved.

Equation (15.7) also implies that the rays $R_{a b}$ and $R_{b a}$ are not the boundaries of the sector where the asymptotic behaviour of $Y_{\nu+\mu}(z, t ; \widetilde{\tau})$ holds. The above discussion repeated for all $a, b$ such that $u_{a}(0)=u_{b}(0)$ proves the third statement of the theorem.

\section{PART IV: Isomonodromy Deformations of system (1.9). Theorem 1.1, Corollary 1.1 and Theorem 1.2}

We have established the theory of coalescence in $\mathcal{U}_{\epsilon_{0}}(0)$, and the corresponding characterisation of the limiting Stokes matrices for the system (1.1) - namely system (2.1) of Section 2 - under Assumption 1 , or equivalently for the system (1.5). We now consider the system (1.8) under Assumption 1, already put in the form (1.9), namely

$$
\frac{d Y}{d z}=\widehat{A}(z, t) Y, \quad \widehat{A}(z, t)=\Lambda(t)+\frac{\widehat{A}_{1}(t)}{z},
$$

and study its isomonodromy deformations. The eigenvalues are taken to be linear in $t$, as in (1.15):

$$
u_{i}(t)=u_{i}(0)+t_{i}, \quad 1 \leq i \leq n .
$$

\section{Structure of Fundamental Solutions in Levelt form at $z=0$}

At any point $t \in \mathcal{U}_{\epsilon_{0}}(0)$, let $\mu_{1}(t), \mu_{2}(t), \ldots, \mu_{n}(t)$ be the (non necessarily distinct) eigenvalues of $\widehat{A}_{1}(t)$, and let $J^{(0)}(t)$ be a Jordan form of $\hat{A}_{1}(t)$, with $\operatorname{diag}\left(J^{(0)}\right)=\operatorname{diag}\left(\mu_{1}, \ldots, \mu_{n}\right)$ (see also (16.3) below). The eigenvalues are decomposed uniquely as,

$$
\mu_{i}(t)=d_{i}^{(0)}(t)+\rho_{i}^{(0)}(t), \quad d_{i}^{(0)}(t) \in \mathbb{Z}, 0 \leq \Re \rho_{i}^{(0)}(t)<1 .
$$

Let $D^{(0)}(t)=\operatorname{diag}\left(d_{1}^{(0)}(t), \ldots, d_{n}^{(0)}(t)\right)$, which is piecewise constant, so that

$$
J^{(0)}(t)=D^{(0)}(t)+S^{(0)}(t)
$$

where $S^{(0)}(t)$ is the Jordan matrix with $\operatorname{diag}\left(S^{(0)}\right)=\operatorname{diag}\left(\rho_{1}^{(0)}, \ldots, \rho_{n}^{(0)}\right)$.

Let $\mathcal{V}$ be an open connected subset of $\mathcal{U}_{\epsilon_{0}}(0)$. In order to write a solution at $z=0$ in Levelt form which is holomorphic on $\mathcal{V}$, we need the following assumption. 
Assumption 2: We assume that $\widehat{A}_{1}(t)$ is holomorphically similar to $J^{(0)}(t)$ on $\mathcal{V}$. This means that there exists an invertible matrix $G^{(0)}(t)$ holomorphic on $\mathcal{V}$ such that

$$
\left(G^{(0)}(t)\right)^{-1} \widehat{A}_{1}(t) G^{(0)}(t)=J^{(0)}(t) .
$$

Assumption 2 in $\mathcal{V}$ implies that the eigenvalues $\mu_{i}(t)$ are holomorphic on $\mathcal{V}$. In the isomonodromic case (to be defined below), Assumption 2 for $\mathcal{V}=\mathcal{U}_{\epsilon_{0}}(0)$ turns out to be equivalent to the vanishing condition (1.20). See Proposition 19.2 below.

Remark 16.1. In order to realise the above assumption it is not sufficient to assume, for example, that the eigenvalues of $\widehat{A}_{1}(t)$ are independent of $t$, as the example $\widehat{A}_{1}(t)=\left(\begin{array}{cc}\mu & t \\ 0 & \mu\end{array}\right)$ shows. Sufficient conditions can be found in the Wasow's book [69], Ch. VII.

With Assumption 2, the following fundamental solutions in Levelt form are found.

A] If $\widehat{A}_{1}(t)$ has distinct eigenvalues at any point of $\mathcal{V}$, it is automatically holomorphically similar to

$$
\widehat{\mu}(t):=\operatorname{diag}\left(\mu_{1}(t), \ldots, \mu_{n}(t)\right) .
$$

A fundamental matrix exists of the form

$$
Y^{(0)}(z, t)=G^{(0)}(t)\left(I+\sum_{l=1}^{\infty} \Psi_{l}(t) z^{l}\right) z^{\widehat{\mu}(t)}
$$

Each matrix $\Psi_{l}(t)$ is holomorphic on $\mathcal{V}$, and the series $I+\sum_{l=1}^{\infty} \Psi_{l}(t) z^{l}$ is absolutely convergent for $|z|$ bounded, defining a holomorphic matrix-valued function in $(z, t)$ on $\{|z|<r\} \times \mathcal{V}$, for any $r>0$.

B] If $\mu_{i}(t)-\mu_{j}(t) \notin \mathbb{Z} \backslash\{0\}$ for any $i \neq j$ and any $t \in \overline{\mathcal{V}}$, then there exists a fundamental matrix

$$
Y^{(0)}(z, t)=G^{(0)}(t)\left(I+\sum_{l=1}^{\infty} \Psi_{l}(t) z^{l}\right) z^{J^{(0)}(t)}
$$

such that $G^{(0)}(t), J^{(0)}(t)$ and each matrix $\Psi_{l}(t)$ are holomoprhic on $\mathcal{V}$, and the series $I+\sum_{l=1}^{\infty} \Psi_{l}(t) z^{l}$ is absolutely convergent for $|z|$ bounded, defining a holomorphic matrix-valued function in $(z, t)$ on $\{|z|<r\} \times \mathcal{V}$, for any $r>0$.

The above forms of the matrix $Y^{(0)}(z, t)$ are obtained by a recursive procedure (see [69]), aimed at constructing a gauge transformation $Y=G^{(0)}(t)\left(I+\sum_{l=1}^{\infty} \Psi_{l}(t) z^{l}\right) \mathcal{Y}$ that reduces the linear system to a simple form $\frac{d \mathcal{Y}}{d z}=\frac{J^{(0)}}{z} \mathcal{Y}$, whose solution $z^{J^{(0)}(t)}$ can be immediately written. In resonant cases, namely when $\mu_{i}(t)-\mu_{j}(t) \in \mathbb{Z} \backslash\{0\}$, this procedure yields a gauge transformation $Y=G^{(0)}(t)\left(I+\sum_{l=1}^{\infty} \Psi_{l}(t) z^{l}\right) \mathcal{Y}$ that reduces the system to the form

$$
\frac{d \mathcal{Y}}{d z}=\frac{1}{z}\left(J^{(0)}(t)+R_{1}(t) z+\cdots+R_{\kappa}(t) z^{\kappa}\right) \mathcal{Y}
$$

where $1 \leq \kappa$ is the maximal integer difference of eigenvalues of $J^{(0)}$, and the $R_{j}(t)$ 's are certain nilpotent matrices (see (17.1) below for more details). These matrix coefficients may be discontinuous in $t$, even if Assumption 2 is made. In order to avoid this, we need the following

(Temporary) Assumption 3 [Resonant Case]: If for some $i \neq j$ it happens that $\mu_{i}(t)-\mu_{j}(t) \in$ $\mathbb{Z} \backslash\{0\}$ at a point $t \in \mathcal{V}$, then we require that $\mu_{i}(t)-\mu_{j}(t)=$ constant $\in \mathbb{Z} \backslash\{0\}$ all over $\mathcal{V}$. If moreover $J^{(0)}(t)$ is not diagonal, then we require that the $d_{i}$ 's, $1 \leq i \leq n$, are constant on $\mathcal{V}$.

Assumptions 3 certainly holds if the eigenvalues $\mu_{1}, \ldots, \mu_{n}$ are independent of $t$ in $\mathcal{V}$, namely in the isomonodromic case of Definition 17.2 below. ${ }^{24}$ Hence, Assumptions 3 is only "temporary" here, being unnecessary in the isomonodromic case.

\footnotetext{
${ }^{24}$ In case we define a deformation to be isomonodromic when the monodromy matrices are constant, this is still true, namely $\mu_{1}, \ldots, \mu_{n}$ are independent of $t$. See Lemma 1 of [11].
} 
When Assumptions 2 and 3 hold together, fundamental matrices in Levelt form can always be constructed in such a way that they are holomorphic on $\mathcal{V}$. Besides the cases A] and B] (which require only Assumption 2), we have the following resonant cases:

C] If $J^{(0)}(t) \equiv \widehat{\mu}(t):=\operatorname{diag}\left(\mu_{1}(t), \mu_{1}(t), \ldots, \mu_{n}(t)\right)$ (eigenvalues non necessarily distinct) then there exists a fundamental matrix

$$
Y^{(0)}(z, t)=G^{(0)}(t)\left(I+\sum_{l=1}^{\infty} \Psi_{l}(t) z^{l}\right) z^{\widehat{\mu}(t)} z^{R^{(0)}(t)},
$$

were the matrix $R^{(0)}(t):=R_{1}(t)+\cdots R_{\kappa}(t)$ has entries $R_{i j}^{(0)}(t) \neq 0$ only if $\mu_{i}(t)-\mu_{j}(t) \in \mathbb{N} \backslash\{0\}$. Moreover, $G^{(0)}(t), \widehat{\mu}(t) R^{(0)}(t)$ and each matrix $\Psi_{l}(t)$ can be chosen holomorphic on $\mathcal{V}$, and the series $I+\sum_{l=1}^{\infty} \Psi_{l}(t) z^{l}$ is absolutely convergent for $|z|$ bounded, defining a holomorphic matrix-valued function in $(z, t)$ on $\{|z|<r\} \times \mathcal{V}$, for any $r>0$.

D] If some $\mu_{i}(t)-\mu_{j}(t) \in \mathbb{Z} \backslash\{0\}$ and $J^{(0)}(t)$ is not diagonal, then there exists a fundamental matrix holomorphic on $\mathcal{V}$,

$$
Y^{(0)}(z, t)=G^{(0)}(t)\left(I+\sum_{l=1}^{\infty} \Psi_{l}(t) z^{l}\right) z^{D^{(0)}} z^{L^{(0)}(t)}
$$

where

$$
L^{(0)}(t):=S^{(0)}(t)+R^{(0)}(t)
$$

$G^{(0)}, S^{(0)}$ are holomorphic on $\mathcal{U}_{\epsilon_{0}}(0)$, and $R^{(0)}$ and the $\Psi_{l}$ 's can be chosen holomorphic on $\mathcal{V}$. The series $I+\sum_{l=1}^{\infty} \Psi_{l}(t) z^{l}$ is absolutely convergent for $|z|$ bounded, defining a holomorphic matrix-valued function in $(z, t)$ on $\{|z|<r\} \times \mathcal{V}$, for any $r>0$.

The structure of $R^{(0)}$ is more conveniently described if the eigenvalues $\mu_{1}, \mu_{2}, \ldots, \mu_{n}$ are re-labelled as follows. Up to a permutation $J^{(0)} \mapsto P^{-1} J^{(0)} P$, which corresponds to $G^{(0)} \mapsto G^{(0)} P$, where $P$ is a permutation matrix, the Jordan blocks structure can be arranged as

$$
J^{(0)}=J_{1}^{(0)} \oplus \cdots \oplus J_{s_{0}}^{(0)}, \quad s_{0} \leq n .
$$

For $i=1,2, \ldots, s_{0}$, each $J_{i}^{(0)}$ has dimension $n_{i}$ (then $n_{1}+\cdots+n_{s_{0}}=n$ ) and has only one eigenvalue $\widetilde{\mu}_{i}$, with structure

$$
\begin{gathered}
J_{i}^{(0)}=\widetilde{\mu}_{i} I_{n_{i}}+H_{n_{i}}, \quad I_{n_{i}}=n_{i} \times n_{i} \text { identity matrix } \\
H_{n_{i}}=0 \text { if } n_{i}=1, \quad H_{n_{i}}=\left[\begin{array}{ccccc}
0 & 1 & & & \\
& 0 & 1 & & \\
& & \ddots & \ddots & \\
& & & 0 & 1 \\
& & & 0
\end{array}\right] \text { if } n_{i} \geq 2 .
\end{gathered}
$$

$\widetilde{\mu}_{1}, \ldots, \widetilde{\mu}_{s_{0}}$ are not necessarily distinct. Let us partition $R^{(0)}$ according to the block structure $n_{1}, \ldots, n_{s_{0}}$. Then $\left[R^{(0)}\right]_{\text {block } i, j} \neq 0$ only if $\widetilde{\mu}_{i}-\widetilde{\mu}_{j} \in \mathbb{N} \backslash\{0\}$, for $1 \leq i \neq j \leq s_{0}$.

Remark 16.2. Also in cases $\mathrm{A}], \mathrm{B}]$ and $\mathrm{C}]$ the fundamental solution can be written in the Levelt form (16.2), with $L^{(0)}=S^{(0)}$ in $\left.\mathrm{A}\right]$ and $\left.\mathrm{B}\right]$, and $L^{(0)}=S^{(0)}+R^{(0)}$ in C].

16.1. Freedom. Let the matrix $J^{(0)}(t)$ be fixed with the convention (16.3). Let Assumptions 2 and 3 hold. The class of normal forms at the Fuchsian singularity $z=0$ with given $J^{(0)}$ is not unique, when some eigenvalues of $\widehat{A}_{1}(t)$ differ by non-zero integers. Let $\kappa$ be the maximal integer difference. Then, if (16.2) is a Levelt form, there are other Levelt forms

$$
\begin{aligned}
\widetilde{Y}^{(0)}(z, t) & =\widetilde{G}^{(0)}(t)\left(I+\sum_{l=1}^{\infty} \widetilde{\Psi}_{l}(t) z^{l}\right) z^{D^{(0)}(t)} z^{\widetilde{L}^{(0)}(t)} \\
& \equiv Y^{(0)}(z, t) \mathfrak{D}(t)
\end{aligned}
$$


where $\mathfrak{D}(t)$ is a connection matrix. From the standard theory of equivalence of Birkhoff normal forms of a given differential system with Fuchsian singularity, it follows that $\mathfrak{D}(t)$ must have the following property

$$
z^{D^{(0)}(t)} z^{L^{(0)}(t)} \mathfrak{D}(t)=\mathfrak{D}_{0}(t)\left(I+\mathfrak{D}_{1}(t) z+\cdots+\mathfrak{D}_{\kappa}(t) z^{\kappa}\right) z^{D^{(0)}(t)} z^{\widetilde{L}^{(0)}(t)},
$$

being $\mathfrak{D}_{0}, \ldots, \mathfrak{D}_{\kappa}$ arbitrary matrices satisfying $\left[\mathfrak{D}_{0}, J^{(0)}\right]=0, \mathfrak{D}_{i j}^{(l)} \neq 0$ only if $\widetilde{\mu}_{i}-\widetilde{\mu}_{j}=l>0$. The connection matrix is then

$$
\mathfrak{D}(t)=\mathfrak{D}_{0}(t)\left(I+\mathfrak{D}_{1}(t)+\cdots+\mathfrak{D}_{k}(t)\right) .
$$

Being $\mathfrak{D}_{0}(t), \ldots, \mathfrak{D}_{\kappa}(t)$ arbitrary, we can choose the subclass of those connection matrices $\mathfrak{D}(t)$ which are holomorphic in $t$. Note that $\mathfrak{D}_{0}$ commutes with $D^{(0)}$. The relation between matrices with $\sim$ and without is as follows:

$$
\begin{array}{r}
\widetilde{G}^{(0)}(t)\left(I+\sum_{l=1}^{\infty} \widetilde{\Psi}_{l}(t) z^{l}\right)= \\
=G^{(0)}(t)\left(I+\sum_{l=1}^{\infty} \Psi_{l}(t) z^{l}\right)\left[\mathfrak{D}_{0}(t)\left(I+\mathfrak{D}_{1}(t) z+\cdots+\mathfrak{D}_{\kappa}(t) z^{\kappa}\right)\right] .
\end{array}
$$

Moreover,

$$
\widetilde{L}^{(0)}=\mathfrak{D}^{-1} L^{(0)} \mathfrak{D}, \quad \widetilde{R}^{(0)}=\mathfrak{D}^{-1} R^{(0)} \mathfrak{D}+\mathfrak{D}^{-1}\left[S^{(0)}, \mathfrak{D}\right] .
$$

Observe that

$$
\widetilde{G}^{(0)}(t)=G^{(0)}(t) \Longleftrightarrow \mathfrak{D}_{0}(t)=I .
$$

\section{Definition of Isomonodromy Deformation of the System (1.9) with Eigenvalues}

The Stokes phenomenon at $z=\infty$ has been already described.

Let $\widetilde{\tau}$ be an admissible direction for $\Lambda(0)$. For the remaining part of the paper, $\mathcal{V}$ will denote an open simply connected subset of a $\widetilde{\tau}$-cell, such that the closure $\overline{\mathcal{V}}$ is also contained in the cell. Let the label $\nu$ satisfy $\tau_{\nu}<\widetilde{\tau}<\tau_{\nu+1}$. The holomorphic fundamental matrices of Section 12, namely $Y_{\sigma}(z, t)$, $\sigma=\nu, \nu+\mu, \nu+2 \mu$, exist and satisfy Corollary 13.1 and Proposition 13.3. Therefore, in particular, they have canonical asymptotics on $\mathcal{S}_{\sigma}(\overline{\mathcal{V}})$, with holomorphic on $\mathcal{V}$ Stokes matrices $\mathbb{S}_{\nu}(t)$ and $\mathbb{S}_{\nu+\mu}(t)$.

Remark 17.1. [Notations] The notation $Y_{\nu}(z, t)$ of Sections 12-14 has been used for the fundamental matrix solutions of the system (1.1), (2.1). We consider now the system (1.9) and use the same notation $Y_{\nu}(z, t)$, with the replacement $G_{0}(t) \mapsto I$ in all the formulae where $G_{0}(t)$ appears.

Definition 17.1. The central connection matrix $C_{\nu}^{(0)}(t)$ is defined by

$$
Y_{\nu}(z, t)=Y^{(0)}(z, t) C_{\nu}^{(0)}(t), \quad z \in \mathcal{R} .
$$

Definition 17.2 (Isomonodromic Deformation in $\mathcal{V}$ ). Let $\mathcal{V}$ be an open connected subset of a $\widetilde{\tau}$-cell, such that $\overline{\mathcal{V}}$ is also contained the cell. A t-deformation of the system (1.9) satisfying Assumption 2 in $\mathcal{V}$ is said to be isomonodromic in $\mathcal{V}$ if the essential monodromy data,

$$
\mathbb{S}_{\nu} \quad \mathbb{S}_{\nu+\mu}, \quad B_{1}=\operatorname{diag}\left(\widehat{A}_{1}\right) ; \quad\left\{\mu_{1}, \ldots, \mu_{n}\right\},
$$

are independent of $t \in \mathcal{V}$, and if there exists a fundamental solution (16.2) (see Remark 16.2), holomorphic in $t \in \mathcal{V}$, such that also the corresponding essential monodromy data

$$
R^{(0)}, \quad C_{\nu}^{(0)}
$$

are independent of $t \in \mathcal{V}$.

Remark 17.2. If $\mu_{1}, \ldots, \mu_{n}$ are independent of $t$ as in Definition 17.2, then Assumption 2 in $B$ implies that also Assumption 3 holds in $\mathcal{V}$. 
The existence of a fundamental solution with constant $R^{(0)}$ implies that the system (1.9) can be reduced to a simpler form (16.1) which is independent of $t \in \mathcal{V}$, namely

$$
\frac{d \mathcal{Y}}{d z}=\frac{1}{z}\left(J^{(0)}+R_{1} z+\cdots+R_{\kappa} z^{\kappa}\right) \mathcal{Y},
$$

where $1 \leq \kappa$ is the maximal integer difference of eigenvalues of $J^{(0)},\left[R_{l}\right]_{\text {block } i, j} \neq 0$ only if $\tilde{\mu}_{i}-\widetilde{\mu}_{j}=l$, $R_{1}+\cdots+R_{\kappa}=R^{(0)}$, with all $R_{l}$ independent of $t \in \mathcal{V}$, and the $\widetilde{\mu}_{i}$ 's are the eigenvalues of $\widehat{A}_{1}(t)$ as arranged in the Jordan from (16.3)-(16.4).

Remark 17.3. There is a freedom in the isomonodromic $R^{(0)}$ and $L^{(0)}$, as in (16.5), for a $t$-independent $\mathfrak{D}$ such that $\tilde{Y}^{(0)}=Y^{(0)} \mathfrak{D}$. Hence, there is a freedom in the isomonodromic central connection matrix, according to

$$
C^{(0)}=\mathfrak{D} \widetilde{C}^{(0)}
$$

We call $\mathcal{C}_{0}\left(J^{(0)}, L^{(0)}\right)$ the group of such transformations $\mathfrak{D}$ which leave $L^{(0)}$ invariant in (16.5). This notation is a slight variation of a notation introduced in [19] for a particular subclass of our systems (1.9), related to Frobenius manifolds.

Remark 17.4. Definition 17.2 is given with reference to some $\nu$. Nevertheless, it implies that it holds for any other $\nu^{\prime}$ in a suitably small $\mathcal{V}^{\prime} \subset \mathcal{V}$. To see this, consider another admissible $\widetilde{\tau}^{\prime} \in\left(\tau_{\nu^{\prime}}, \tau_{\nu^{\prime}+1}\right)$, and define $\mathcal{S}_{\nu^{\prime}+k \mu}(t), Y_{\nu^{\prime}+k \mu}(z, t)$ in the usual way, for $t$ in the intersection of $\mathcal{V}$ with a $\widetilde{\tau}^{\prime}$-cell. ${ }^{25}$ Call $\mathcal{V}^{\prime}$ the intersection. Now, there is a finite product of Stokes factors $K_{1}(t) \cdots K_{M}(t)(M \leq$ number of basic Stokes rays of $\Lambda(t))$ such that $Y_{\nu}(z, t)=Y_{\nu^{\prime}}(z, t) K_{1}(t) \cdots K_{M}(t), t \in \mathcal{V}^{\prime}$. The Stokes matrices $\mathbb{S}_{\nu}(t)$ and $\mathbb{S}_{\nu+\mu}(t)$ are determined uniquely by their factors, and conversely a Stokes matrix determines uniquely the factors of a factorization of the prescribed structure (see the proof of Theorem 15.1, or section 4 of [2], point D). Moreover, the product $K_{1}(t) \cdots K_{M}(t)$ appears in the factorization of $\mathbb{S}_{\nu}$ or $\mathbb{S}_{\nu+\mu}$. Hence, if $\mathbb{S}_{\nu}$ and $\mathbb{S}_{\nu+\mu}$ do not depend on $t \in \mathcal{V}$ for a certain $\nu$, also $\mathbb{S}_{\nu^{\prime}}$ and $\mathbb{S}_{\nu^{\prime}+\mu}$ do not depend on $t \in \mathcal{V}^{\prime} \subset \mathcal{V}$. Thus, the same is true for $C_{\nu^{\prime}}^{(0)}$.

Lemma 17.1. Let the deformation be isomonodromic in $\mathcal{V}$ as in Definition 17.2 (here it is not necessary to suppose that $\mathcal{V}$ is in a cell, since we are considering solutions at $z=0)$. Let Assumption 2 hold in $\mathcal{U}_{\epsilon_{0}}(0)$, namely let $\widehat{A}_{1}(t)$ be holomorphically equivalent to $J^{(0)}$ in $\mathcal{U}_{\epsilon_{0}}(0)$. Then:

i) $\mu_{1}, \ldots, \mu_{n}, D^{(0)}, S^{(0)}$ and $J^{(0)}$ are independent of $t$ in $\mathcal{U}_{\epsilon_{0}}(0)$.

ii) Any fundamental matrix (also non-isomonodromic ones) in Levelt form $Y^{(0)}(z, t)=G^{(0)}(t)(I+$ $\left.\sum_{l} \Psi_{l}(t) z^{l}\right) z^{D} z^{L^{(0)}(t)}$, which is holomorphic of $t \in \mathcal{V}$, is also holomorphic on the whole $\mathcal{U}_{\epsilon_{0}}(0)$.

iii) If $R^{(0)}$ (i.e $\left.L^{(0)}\right)$ is independent of $t$ in $\mathcal{V}$, then it is independent of $t$ in $\mathcal{U}_{\epsilon_{0}}(0)$.

Proof: i) That $\mu_{1}, \ldots, \mu_{n}, D^{(0)}, S^{(0)}, J^{(0)}$ are constant in $\mathcal{U}_{\epsilon_{0}}(0)$ follows from the fact that $\mu_{1}, \ldots, \mu_{n}$ are constant in $\mathcal{V}$, and that $G^{(0)}(t)$, and so the $\mu_{1}, \ldots, \mu_{n}$, are holomorphic on $\mathcal{U}_{\epsilon_{0}}(0)$. So $\mu_{1}, \ldots, \mu_{n}$ are constant in $\mathcal{U}_{\epsilon_{0}}(0)$.

ii) Since $\mu_{1}, \ldots, \mu_{n}$ are constant in $\mathcal{U}_{\epsilon_{0}}(0)$, and $\Lambda(t)$ and $\widehat{A}_{1}(t)$ are holomorphic, the recursive standard procedure which yields the Birkhoff normal form at $z=0$ allows to choose $\Psi_{l}(t)$ 's and $R^{(0)}(t)$ holomorphic on $\mathcal{U}_{\epsilon_{0}}(0)$.

iii) That $R^{(0)}$ is independent of $t \in \mathcal{U}_{\epsilon_{0}}(0)$ follows from the fact that $R^{(0)}(t)$ is holomoprhic on $\mathcal{U}_{\epsilon_{0}}(0)$ and constant on $\mathcal{V}$.

Proposition 17.1. Let the deformation of the system (1.9) be isomonodromic in $\mathcal{V}$ as in Definition 17.2 (here it is not necessary to assume that $\mathcal{V}$ is contained in a cell). Let Assumption 2 hold in $\mathcal{U}_{\epsilon_{0}}(0)$, namely let $\widehat{A}_{1}(t)$ be holomorphically equivalent to $J^{(0)}=D^{(0)}+S^{(0)}$ in $\mathcal{U}_{\epsilon_{0}}(0)$. Consider the system

$$
\frac{d Y}{d z}=\widehat{A}(z, 0) Y
$$

\footnotetext{
${ }^{25}$ Note that there may be more than one choices for $\mathcal{S}_{\nu^{\prime}+k \mu}, Y_{\nu^{\prime}+k \mu}(z, t)$, depending on the neighbourhood of $t$ considered. See Remark 11.1.
} 
and a fundamental solution in the Levelt form

$$
\stackrel{\circ}{Y}^{(0)}(z)=\stackrel{\circ}{G}^{(0)} \stackrel{\circ}{G}(z) z^{D^{(0)}} z^{\circ}, \quad \stackrel{G}{G}(z)=I+\mathcal{O}(z),
$$

with $\stackrel{\circ}{L}=S^{(0)}+\stackrel{\circ}{R}$. Here $\stackrel{\circ}{R}$ is obtained by reducing (17.2) to a Birkhoff normal form at $z=0$. Then, there exists an isomonodromic fundamental solution of (1.9), call it $Y_{\text {isom }}^{(0)}(z, t)$, with the same monodromy exponent $\stackrel{\circ}{L}$ and Levelt form

$$
Y_{\text {isom }}^{(0)}(z, t)=G^{(0)}(t) G_{i s o m}(z, t) z^{D^{(0)}} z^{\llcorner},
$$

with $G_{i s o m}(z, t)=I+\sum_{k=1}^{\infty} \Psi_{l}(t) z^{l}$, holomorphic on $\mathcal{U}_{\epsilon_{0}}(0)$, such that

$$
\stackrel{\circ}{Y}^{(0)}(z)=Y_{\text {isom }}^{(0)}(z, 0) \text {. }
$$

Proof: We prove the proposition in two steps.

- The first step is the following

Lemma 17.2. Let the deformation be isomonodromic in $\mathcal{V}$ as in Definition 17.2 (here it is not necessary to assume that $\mathcal{V}$ is contained in a cell). Let $\widehat{A}_{1}(t)$ be holomorphically equivalent to $J^{(0)}$ in $\mathcal{U}_{\epsilon_{0}}(0)$. For any holomorphic fundamental solution in Levelt form

$$
Y(z, t)=G(t) H(z, t) z^{D^{(0)}} z^{L^{(0)}(t)}, \quad H(z, t)=I+\sum_{l=1}^{\infty} h_{l}(t) z^{l},
$$

with monodromy exponent $L^{(0)}(t)$, there exists an isomonodromic $Y^{(0)}(z, t)$, with monodromy exponent equal to $L^{(0)}(0)$, in the Levelt form

$$
Y^{(0)}(z, t)=G^{(0)}(t) G(z, t) z^{D^{(0)}} z^{L^{(0)}(0)}, \quad G(z, t)=I+\sum_{l=1}^{\infty} \Psi_{l}(t) z^{l},
$$

such that $Y^{(0)}(z, 0)=Y(z, 0)$.

To prove this Lemma, consider an isomonodromic fundamental solution, which exists by assumption, say

$$
\widetilde{Y}^{(0)}(z, t)=\widetilde{G}^{(0)}(t) \widetilde{G}(z, t) z^{D^{(0)}} z^{(0)}, \quad \widetilde{G}(z, t)=I+\sum_{l=1}^{\infty} \widetilde{\Psi}_{l}(t) z^{l},
$$

with $t$-independent monodromy exponent $\widetilde{L}^{(0)}$ and $t$-independent connection matrix defined by

$$
Y_{\nu}(z, t)=\widetilde{Y}^{(0)}(z, t) \widetilde{C}_{\nu}^{(0)}
$$

Then, there exists a holomorphic invertible connection matrix $\mathfrak{D}(t)$ such that

$$
Y(z, t)=\tilde{Y}^{(0)}(z, t) \mathfrak{D}(t) .
$$

Hence,

$$
\mathfrak{D}_{0}(t)\left(I+\mathfrak{D}_{1}(t) z+\cdots+\mathfrak{D}_{\kappa}(t) z^{\kappa}\right) z^{D^{(0)}} z^{L^{(0)}(t)}=z^{D^{(0)}} z^{\widetilde{L}^{(0)}} \mathfrak{D}(t)
$$

with $\mathfrak{D}(t)=\mathfrak{D}_{0}(t)\left(I+\mathfrak{D}_{1}(t)+\cdots+\mathfrak{D}_{\kappa}(t)\right)$. Observe that $z^{D^{(0)} z^{L^{(0)}(0)}}$ and $z^{D^{(0)}} z^{\widetilde{L}^{(0)}}$ are fundamental solutions of two Birkhoff normal forms of (17.2), related by (17.4) with $t=0$, namely

$$
\mathfrak{D}_{0}(0)\left(I+\mathfrak{D}_{1}(0) z+\cdots+\mathfrak{D}_{\kappa}(0) z^{\kappa}\right) z^{D^{(0)}} z^{L^{(0)}(0)}=z^{D^{(0)}} z^{\widetilde{L}^{(0)}} \mathfrak{D}(0) .
$$

Therefore, the isomonodromic fundamental solution we are looking for is

$$
Y^{(0)}(z, t):=\widetilde{Y}^{(0)}(z, t) \mathfrak{D}(0)=Y(z, t) \mathfrak{D}(t)^{-1} \mathfrak{D}(0) .
$$

- Second step. Consider a fundamental solution of (17.2) in the Levelt form

$$
\stackrel{\circ}{Y}^{(0)}(z)=\stackrel{\circ}{G}^{(0)} \stackrel{\circ}{G}(z) z^{D^{(0)}} z^{\stackrel{\circ}{L}}
$$

where $\stackrel{\circ}{L}=S^{(0)}+\stackrel{\circ}{R}, \stackrel{\circ}{R}=\sum_{l=1}^{\kappa} \stackrel{\circ}{R}_{l}$. The $\stackrel{\circ}{R}_{l}, l=1,2, \ldots, \kappa$, are coefficients of a simple gauge equivalent form(16.1), with $t=0$, of (17.2). It can be proved that there is a form (16.1) for the system (1.9), 
with coefficients $R_{l}(t)$, such that the $\stackrel{R}{l}_{l}$ 's coincide with the values $R_{l}(0)$ 's at $t=0$. Moreover, the $R_{l}(t)$ 's are holomorphic on $\mathcal{U}_{\epsilon_{0}}(0)$. This fact follows from the recursive procedure which yileds the gauge transformation from (1.9) to (16.1). Therefore, there exists a holomorphic exponent $L^{(0)}(t)$ such that $L^{(0)}(0)=\stackrel{\circ}{L}$. Consider an isomonodromic fundamental solution $Y^{(0)}(z, t)$ of Lemma 17.2, with exponent $L^{(0)}(0)=\stackrel{\circ}{L}$. Since $Y^{(0)}(z, 0)$ is a fundamental solution of $(17.2)$, there exists an invertible and constant connection matrix $C$ such that

$$
Y^{(0)}(z, 0) C=\stackrel{\circ}{Y}^{(0)}(z) .
$$

Now, $C \in \mathcal{C}_{0}\left(J^{(0)}, \stackrel{\circ}{L}\right.$ ) (cf. Remark 17.3), because $Y^{(0)}(z, 0)$ and $\stackrel{\circ}{Y}^{(0)}(z)$ have the same monodromy exponent. This implies that

$$
\begin{aligned}
Y^{(0)}(z, t) C \quad & =G^{(0)} G(z, t) z^{D^{(0)}} z^{\stackrel{\circ}{L}} C= \\
& =G^{(0)} G(z, t) C_{0}\left(I+C_{1} z+\cdots+C_{\kappa} z^{\kappa}\right) z^{D^{(0)}} z^{\stackrel{\circ}{L}}, \quad C=C_{0}\left(I+C_{1}+\cdots+C_{\kappa}\right) .
\end{aligned}
$$

Moreover, also $Y^{(0)}(z, t) C$ is isomonodromic. Therefore, the solution we are looking for is $Y_{i s o m}^{(0)}(z, t):=$ $Y^{(0)}(z, t) C$.

\section{Isomonodromy Deformation Equations}

Let

$$
\Omega(z, t):=\sum_{k=1}^{n} \Omega_{k}(z, t) d t_{k}, \quad \Omega_{k}(z, t):=z E_{k}+\left[F_{1}(t), E_{k}\right] .
$$

Here $E_{k}$ is the matrix with all entries equal to zero, except for $\left(E_{k}\right)_{k k}=1$, and $\left(F_{1}\right)_{a b}=-\left(\widehat{A}_{1}\right)_{a b} /\left(u_{a}-\right.$ $\left.u_{b}\right)$, so that

$$
\left[F_{1}(t), E_{k}\right]=\left(\frac{\left(\widehat{A}_{1}(t)\right)_{a b}\left(\delta_{a k}-\delta_{b k}\right)}{u_{a}(t)-u_{b}(t)}\right)_{a, b=1 . . n}=\left(\begin{array}{ccccc}
0 & 0 & \frac{-\left(\widehat{A}_{1}\right)_{1 k}}{u_{1}-u_{k}} & 0 & 0 \\
0 & 0 & \vdots & 0 & 0 \\
\frac{\left(\widehat{A}_{1}\right)_{k 1}}{u_{k}-u_{1}} & \cdots & 0 & \cdots & \frac{\left(\widehat{A}_{1}\right)_{k n}}{u_{k}-u_{n}} \\
0 & 0 & \vdots & 0 & 0 \\
0 & 0 & \frac{-\left(\widehat{A}_{1}\right)_{n k}}{u_{n}-u_{k}} & 0 & 0
\end{array}\right)
$$

Let $d f(z, t):=\sum_{i=1}^{n} \partial f(z, t) / \partial t_{i} d t_{i}$.

Theorem 18.1. If the deformation of the system (1.9) is isomonodromic in $\mathcal{V}$ as in Definition 17.2, then an isomonodromic $Y^{(0)}(z, t)$ and the $Y_{\sigma}(z, t)$ 's, for $\sigma=\nu, \nu+\mu, \nu+2 \mu$, satisfy the total differential system

$$
d Y=\Omega(z, t) Y .
$$

Conversely, if the $t$-deformation satisfies assumptions 2 and 3 in $\mathcal{V}$, and if a fundamental solution $Y^{(0)}(z, t)$ in Levelt form at $z=0$, and the canonical solution $Y_{\sigma}(z, t), \sigma=\nu, \nu+\mu, \nu+2 \mu$ at $z=\infty$, satisfy the total differential system (18.2), then the deformation is isomonodromic in $\mathcal{V}$.

Proof: The proof is done in the same way as for Theorem 3.1 at page 322 in [44]. In [44] the proof is given for non resonant $\widehat{A}_{1}(t)$, but it can be repeated in our case with no changes, except for the assumptions 2, 3. ${ }^{26}$ The matrix valued differential form $\Omega(z, t)$ turns out to be still as in formula (3.8) and (3.14) of [44], which in our case becomes,

$$
\Omega(z, t)=\left[\left(I+\sum_{k=1}^{\infty} F_{k}(t) z^{-k}\right) d \Lambda(t) z\left(I+\sum_{k=1}^{\infty} F_{k}(t) z^{-k}\right)^{-1}\right]_{s i n g},
$$

\footnotetext{
${ }^{26}$ The result was announced in [56] and not proved. It can also be proved by the methods of [42], since the requirement that $\mu_{1}, \ldots, \mu_{n}, R^{(0)}$ and $C^{(0)}$ are constant is equivalent to having an isoprincipal deformation.
} 
where $[\cdots]_{\operatorname{sing}}$ stands for the singular terms at infinity, namely the terms with powers $z^{j}, j \geq 0$, in the above formal expansion. This is

$$
\Omega(z, t)=d \Lambda(t) z+\left[F_{1}(t), d \Lambda(t)\right] .
$$

Therefore,

$$
\Omega_{k}(z, t)=\frac{\partial \Lambda(t)}{\partial t_{k}} z+\left[F_{1}(t), \frac{\partial \Lambda(t)}{\partial t_{k}}\right]=E_{k}+\left[F_{1}(t), E_{k}\right] .
$$

In the last step we have used the fact that $\Lambda(t)=\operatorname{diag}\left(u_{1}(t), u_{2}(t), \ldots, u_{n}(t)\right)$, with eigenvalues (1.15). In the domain $\mathcal{V}$ the eigenvalues are distinct, so the off-diagonal entries of $F_{1}$ are:

$$
\left(F_{1}\right)_{a b}=\frac{\left(\widehat{A}_{1}\right)_{a b}}{u_{b}-u_{a}}, \quad 1 \leq a \neq b \leq n .
$$

Hence,

$$
\Omega_{k}(z, t)=E_{k} z+\left(\frac{\widehat{A}_{a b}^{(1)}}{u_{b}(t)-u_{a}(t)} \frac{\partial}{\partial t_{k}}\left(u_{b}(t)-u_{a}(t)\right)\right)_{a, b=1}^{n} .
$$

Finally, observe that $\frac{\partial}{\partial t_{k}}\left(u_{b}(t)-u_{a}(t)\right)=\frac{\partial}{\partial t_{k}}\left(t_{b}-t_{a}\right)=\delta_{k b}-\delta_{k a}$. The proof is concluded.

Corollary 18.1. If the deformation of the system (1.9) is isomonodromic in $\mathcal{V}$ as in Definition 17.2, then $G^{(0)}(t)$ satisfies

$$
d G^{(0)}=\Theta^{(0)}(t) G^{(0)},
$$

where

$$
\Theta^{(0)}(t)=\Omega(0, t)=\sum_{k}\left[F_{1}(t), E_{k}\right] d t_{k} .
$$

More explicitly,

$$
\Theta^{(0)}(t)=\left(\frac{\widehat{A}_{a b}^{(1)}}{u_{a}(t)-u_{b}(t)}\left(d t_{a}-d t_{b}\right)\right)_{a, b=1}^{n}
$$

Proof: Substitute $Y^{(0)}$ into (18.2) an compare coefficients of equal powers of $z$. Equation (18.3) comes form the coefficient of $z^{0}$.

Proposition 18.1. If the deformation is isomonodromic in $\mathcal{V}$ as in Definition 17.2, then

$$
d \widehat{A}=\frac{\partial \Omega}{\partial z}+[\Omega, \widehat{A}] .
$$

Proof: Let the deformation be isomonodromic. Then, by Theorem 18.1, equations (1.9) and (18.2) are compatible. The compatibility condition is (18.4).

Note that (18.4) is a necessary condition of isomonodromicity, but not sufficient in case of resonances (sufficiency can be proved if the eigenvalues of $\widehat{A}_{1}$ do not differ by integers, cf. [44]). Explicitly, (18.4) is

$$
\left\{\begin{aligned}
{\left[E_{k}, \widehat{A}_{1}\right] } & =\left[\Lambda,\left[F_{1}, E_{k}\right]\right], \quad k=1, \ldots, n, \\
d \widehat{A}_{1} & =\left[\Theta^{(0)}, \widehat{A}_{1}\right] .
\end{aligned}\right.
$$

The first $n$ equations are automatically satisfied by definition of $F_{1}$. The last equation in components is

$$
\frac{\partial \widehat{A}_{1}}{\partial t_{k}}=\left[\left[F_{1}, E_{k}\right], \widehat{A}_{1}\right],
$$

where $\left[F_{1}, E_{k}\right]$ is in (18.1). 
19. Holomorphic Extension of Isomonodromy Deformations to $\mathcal{U}_{\epsilon_{0}}(0)$ and Theorem 1.1

Lemma 19.1. In case the eigenvalues of $\Lambda(t)$ are as in (1.15) and $\widehat{A}_{1}(t)$ is holomorphic on $\mathcal{U}_{\epsilon_{0}}(0)$, then $\Omega(z, t)$ is holomoprhic (in $t$ ) on $\mathcal{U}_{\epsilon_{0}}(0)$ if and only if

$$
\left(\widehat{A}_{1}\right)_{a b}(t)=\mathcal{O}\left(u_{a}(t)-u_{b}(t)\right) \equiv \mathcal{O}\left(t_{a}-t_{b}\right)
$$

whenever $u_{a}(t)$ and $u_{b}(t)$ coalesce as $t$ tends to a point of $\Delta \subset \mathcal{U}_{\epsilon_{0}}(0)$.

Also $\Theta^{(0)}(t)$ of Corollary 18.1 is holomorphic on $\mathcal{U}_{\epsilon_{0}}(0)$ if and only if (19.1) holds.

Proof: By (18.1), $\Omega(z, t)$ and $\Theta^{(0)}(t)$ are continuous at $t_{\Delta} \in \Delta$ if and only if (19.1) holds for those $u_{a}(t)$, $u_{b}(t)$ coalescing at $t_{\Delta} \in \Delta$. Hence, any point of $\Delta$ is a removable singularity if and only if (19.1) holds.

Proposition 19.1. The system

$$
\begin{aligned}
& d \widehat{A}=\frac{\partial \Omega}{\partial z}+[\Omega, \widehat{A}], \\
& d G^{(0)}=\Theta^{(0)}(t) G^{(0)},
\end{aligned}
$$

with $\widehat{A}_{1}$ holomorphic satisfying condition (19.1) on $\mathcal{U}_{\epsilon_{0}}(0)$, is Frobenius integrable for $t \in \mathcal{U}_{\epsilon_{0}}(0)$.

The proof is as in [44]. It holds also in our case, because the algebraic relations are the same as in our case, no matter if $\widehat{A}_{1}$ is resonant (see e.g. Example 3.2 in [44]).

Write $\Theta^{(0)}=\sum_{k} \Theta_{k}^{(0)} d t_{k}$. Since (18.3) is integrable, the compatibility of equations holds:

$$
\frac{\partial \Theta_{j}^{(0)}}{\partial t_{i}}-\frac{\partial \Theta_{i}^{(0)}}{\partial t_{j}}=\Theta_{i}^{(0)} \Theta_{j}^{(0)}-\Theta_{j}^{(0)} \Theta_{i}^{(0)}
$$

Proposition 19.2. Let the deformation of the system (1.9) be isomonodromic in $\mathcal{V}$ as in Definition 17.2, with $\Lambda(t)$ is as in (1.15) and $\widehat{A}_{1}(t)$ holomorphic on $\mathcal{U}_{\epsilon_{0}}(0)$. Then, $\widehat{A}_{1}(t)$ is holomorphically similar to $J^{(0)}$ in the whole $\mathcal{U}_{\epsilon_{0}}(0)$ if and only if (19.1) holds as $t$ tends to points of $\Delta \subset \mathcal{U}_{\epsilon_{0}}(0)$. In other words, if the deformation is isomonodromic in $\mathcal{V}$ with holomorphic $\widehat{A}_{1}(t)$, then Assumption 2 in the whole $\mathcal{U}_{\epsilon_{0}}(0)$ is equivalent to (19.1).

Proof: Let $\widehat{A}_{1}(t)$ be holomorphic and let $(19.1)$ hold, so that $\Theta^{(0)}(t)$ is holomorphic on $\mathcal{U}_{\epsilon_{0}}(0)$ by Lemma 19.1. The linear Pfaffian systems $d G^{(0)}=\Theta^{(0)}(t) G^{(0)}$ and $d\left[\left(G^{(0)}\right)^{-1}\right]=-\left(G^{(0)}\right)^{-1} \Theta^{(0)}(t)$ are integrable in $\mathcal{U}_{\epsilon_{0}}(0)$, with holomorphic coefficients $\Theta^{(0)}(t)$. Then, a solution $G^{(0)}(t)$ has analytic continuation onto $\mathcal{U}_{\epsilon_{0}}(0)$. We take a solution satisfying $\left(G^{(0)}(t)\right)^{-1} \widehat{A}_{1}(t) G^{(0)}(t)=J^{(0)}$ for $t \in \mathcal{V}$, which then has analytic continuation onto $\mathcal{U}_{\epsilon_{0}}(0)$ as a holomorphic invertible matrix. Hence, $\left(G^{(0)}(t)\right)^{-1} \widehat{A}_{1}(t) G^{(0)}(t)=J^{(0)}$ holds in $\mathcal{U}_{\epsilon_{0}}(0)$ with holomorphic $G^{(0)}(t)$. Conversely, suppose that Assumption 2 holds in $\mathcal{U}_{\epsilon_{0}}(0)$. Then $G^{(0)}(t)$ and $G^{(0)}(t)^{-1}$ are holomorphic on $\mathcal{U}_{\epsilon_{0}}(0)$. Therefore, also $\Theta^{(0)}(t)$ is holomorphic on $\mathcal{U}_{\epsilon_{0}}(0)$, because $\Theta^{(0)}(t)=d G^{(0)} \cdot\left(G^{(0)}\right)^{-1}$ defines the analytic continuation of $\Theta^{(0)}(t)$ on $\mathcal{U}_{\epsilon_{0}}(0)$. Then (19.1) holds, by Lemma 19.1.

Summarising, if $\Lambda(t)$ is as in (1.15) and $\widehat{A}_{1}(t)$ is holomorphic on $\mathcal{U}_{\epsilon_{0}}(0)$, if the deformation is isomonodromic in a simply connected subset $\mathcal{V}$ of a cell, s.t. $\overline{\mathcal{V}} \subset$ cell, then it suffices to assume that $\widehat{A}_{1}(t)$ is holomorphically similar to a Jordan form $J^{(0)}(t)$ in $\mathcal{U}_{\epsilon_{0}}(0)$, or equivalently that (19.1) holds at $\Delta \subset \mathcal{U}_{\epsilon_{0}}(0)$, in order to conclude that the system

$$
\begin{aligned}
& d Y=\Omega(z, t) Y, \\
& d G^{(0)}=\Theta^{(0)}(t) G^{(0)},
\end{aligned}
$$


has holomorphic coefficients on $\mathcal{R} \times \mathcal{U}_{\epsilon_{0}}(0)$. The integrability/compatibility condition of (18.2) is

$$
\frac{\partial \Omega_{j}}{\partial t_{i}}-\frac{\partial \Omega_{i}}{\partial t_{j}}=\Omega_{i} \Omega_{j}-\Omega_{j} \Omega_{i} .
$$

If this relation is explicitly written, it turns out to be equivalent to (19.2). Hence, being (18.3) integrable, also the linear Pfaffian system (18.2) is integrable, with coefficients holomorphic in $\mathcal{U}_{\epsilon_{0}}(0)$. Therefore, due to linearity, any solution $Y(z, t)$ can be $t$-analytically continued along any curve in $\mathcal{U}_{\epsilon_{0}}(0)$, for $z$ fixed.

Corollary 19.1. Let the deformation be isomonodromic in a simply connected subset $\mathcal{V}$ of a cell, s.t. $\overline{\mathcal{V}} \subset$ cell. If $\widehat{A}_{1}(t)$ is holomorphically similar to a Jordan form $J^{(0)}$ in $\mathcal{U}_{\epsilon_{0}}(0)$, or equivalently if (19.1) holds in $\mathcal{U}_{\epsilon_{0}}(0)$, then the $Y_{\sigma}(z, t)$ 's, $\sigma=\nu, \nu+\mu, \nu+2 \mu$, together with an isomonodromic $Y^{(0)}(z, t)$, can be $t$-analytically continued as single valued holomorphic functions on $\mathcal{U}_{\epsilon_{0}}(0)$.

Proof: If the deformation is isomonodromic, by Theorem 18.1 the system (1.9),(18.2) is a completely integrable linear Pfaffian system (compatibility conditions (18.4) and (19.3) hold), with common solutions $Y_{\sigma}(z, t)$ 's, $\sigma=\nu, \nu+\mu, \nu+2 \mu$, and $Y^{(0)}(z, t)$. If $\widehat{A}_{1}(t)$ is holomorphically similar to a Jordan form $J^{(0)}$ in $\mathcal{U}_{\epsilon_{0}}(0)$, or equivalently if (19.1) holds in $\mathcal{U}_{\epsilon_{0}}(0)$, then the coefficients are holomorphic in $\mathcal{U}_{\epsilon_{0}}(0)$, by Proposition 19.2. In particular, since $Y_{\sigma}(z, t)$ 's, $\sigma=\nu, \nu+\mu, \nu+2 \mu$, and $Y^{(0)}(z, t)$ solve (18.2), they can be $t$-analytically continued along any curve in $\mathcal{U}_{\epsilon_{0}}(0)$.

Remark 19.1. Corollary 19.1 can be compared with the result of [55]. It is always true that the $Y_{\sigma}(t, z)$ 's and $Y^{(0)}(t, z)$ can be t-analytically continued on $\mathcal{T}$ as a meromorphic function, where (in our case):

$$
\mathcal{T}=\text { universal covering of } \mathbb{C}^{n} \backslash \Delta_{\mathbb{C}^{n}} .
$$

Here $\Delta_{\mathbb{C}^{n}}$ is the locus of $\mathbb{C}^{n}$ where eigenvalues of $\Lambda(t)$ coalesce. It is a locus of "fixed singularities" (including branch points and essential singularities) of $\Omega(z, t)$ and of any solution of $d Y=\Omega Y$. The movable singularities of $\Omega(z, t), Y_{\sigma}(t, z)$ and $Y^{(0)}(t, z)$ outside the locus are poles and constitute the zeros of the Jimbo-Miwa isomonodromic $\tau$-function [55]. Here, we have furthermore assumed that $\widehat{A}_{1}$ is holomorphic in $\mathcal{U}_{\epsilon_{0}}(0)$ and that (19.1) holds. This fact has allowed us to conclude that $Y_{\sigma}(z, t)$ 's, $\sigma=\nu, \nu+\mu, \nu+2 \mu$, and $Y^{(0)}(z, t)$ are $t$-holomorphic in $\mathcal{U}_{\epsilon_{0}}(0)$.

In order to prove Theorem 1.1, we need a last ingredient, namely the analyticity at $\Delta$ of the coefficients $F_{k}(t)$ of the formal solution computed away from $\Delta$.

Proposition 19.3. Let the deformation of the system (1.9) be isomonodromic in a simply connected subset $\mathcal{V}$ of a cell, s.t. $\overline{\mathcal{V}} \subset$ cell. If $\widehat{A}_{1}(t)$ is holomorphically similar to a Jordan form $J^{(0)}$ in $\mathcal{U}_{\epsilon_{0}}(0)$, or equivalently if (19.1) holds in $\mathcal{U}_{\epsilon_{0}}(0)$, then the coefficients $F_{k}(t), k \geq 1$, of a formal solution of (1.9)

$$
Y_{F}(z, t)=\left(I+\sum_{k=1}^{\infty} F_{k}(t) z^{-k}\right) z^{B_{1}} e^{\Lambda(t) z},
$$

are holomorphic on $\mathcal{U}_{\epsilon_{0}}(0)$.

Proof: Recall that

$$
\begin{aligned}
\left(F_{1}\right)_{a b}(t) & =\frac{\left(\widehat{A}_{1}\right)_{a b}(t)}{u_{b}(t)-u_{a}(t)}, \quad a \neq b, \\
\left(F_{1}\right)_{a a}(t) & =-\sum_{b \neq a}\left(\widehat{A}_{1}\right)_{a b}(t)\left(F_{1}\right)_{b a}(t) .
\end{aligned}
$$

If by assumption (19.1) holds, the above formulas imply that $F_{1}(t)$ is holomorphic in $\mathcal{U}_{\epsilon_{0}}(0)$, because the singularities at $\Delta$, i.e. for $u_{a}(t)-u_{b}(t) \rightarrow 0$, become removable. Since the asymptotics corresponding 
to (19.4) is uniform in a compact subset $K$ of a simply connected open subset of a cell, we substitute it into $d Y=\Omega(z, t) Y$, with

$$
\Omega(z, t)=z d \Lambda(t)+\left[F_{1}(t), d \Lambda(t)\right]
$$

By comparing coefficients of powers of $z^{-l}$ we obtain

$$
\left[F_{l+1}(t), d \Lambda(t)\right]=\left[F_{1}(t), d \Lambda(t)\right] F_{l}(t)-d F_{l}(t), \quad l \geq 1
$$

In components of the differential $d$, this becomes a recursive relation (use $\partial \Lambda(t) / \partial t_{i}=E_{i}$ ):

$$
\left[F_{l+1}(t), E_{i}\right]=\left[F_{1}(t), E_{i}\right] F_{l}(t)-\frac{\partial F_{l}(t)}{\partial t_{i}}
$$

with,

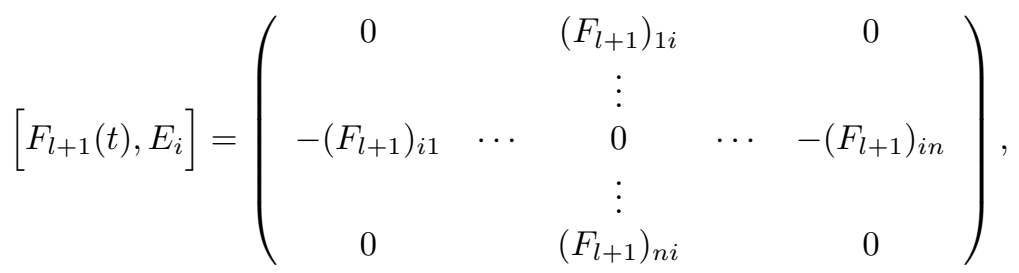

The diagonal element $(i, i)$ is zero. Therefore, (19.5) recursively determines $F_{l+1}$ as a function of $F_{l}, F_{l-1}, \ldots, F_{1}$, except for the diagonal $\operatorname{diag}\left(F_{l+1}\right)$. On the other hand, the diagonal elements are determined by the off-diagonal elements according to the already proved formula,

$$
l\left(F_{l+1}\right)_{a a}(t)=-\sum_{b \neq a}\left(\widehat{A}_{1}\right)_{a b}(t)\left(F_{l}\right)_{b a}(t) .
$$

Let us start with $l+1=2$. Since $F_{1}$ is holomorphic, the above formulae $(19.5),(19.6)$ imply that $F_{2}$ is holomorphic. Then, by induction the same formulae imply that all the $F_{l+1}(t)$ are holomorphic.

Corollary 19.1 means that assumption 2) of Theorem 14.1 applies, while Proposition 19.3 means that assumption 1) applies. This, together with Proposition 17.1, proves the following theorem, which is indeed our Theorem 1.1.

Theorem 19.1 (Theorem 1.1.). Let $\Lambda(t)$ and $\widehat{A}_{1}(t)$ be holomorphic on $\mathcal{U}_{\epsilon_{0}}(0)$, with eigenvalues as in (1.15). If the deformation of the system (1.9) is isomonodromic on a simply connected subset $\mathcal{V}$ of a cell, such that $\overline{\mathcal{V}}$ is in the cell, and if $\widehat{A}_{1}(t)$ is holomorphically similar to a Jordan form $J^{(0)}$ in $\mathcal{U}_{\epsilon_{0}}(0)$, or equivalently the vanishing condition

$$
\left(\widehat{A}_{1}\right)_{a b}(t)=\mathcal{O}\left(u_{a}(t)-u_{b}(t)\right) \equiv \mathcal{O}\left(t_{a}-t_{b}\right),
$$

holds at points of $\Delta$ in $\mathcal{U}_{\epsilon_{0}}(0)$, then Theorem 14.1 and Corollary 14.2 hold (with $G_{0}(t) \mapsto I$, see Remark 17.1), so that $\mathcal{G}_{\sigma}(z, t)=Y_{\sigma}(z, t) e^{\Lambda(t)} z^{-B_{1}(t)}, \sigma=\nu, \nu+\mu, \nu+2 \mu$, maintains the canonical asymptotics

$$
\mathcal{G}_{\sigma}(z, t) \sim I+\sum_{k=1}^{\infty} F_{k}(t) z^{-k}, \quad z \rightarrow \infty \text { in } \widehat{\mathcal{S}}_{\sigma},
$$

for any $t \in \mathcal{U}_{\epsilon_{1}}(0)$ and any $\epsilon_{1}<\epsilon_{0}$. The Stokes matrices,

$$
\mathbb{S}_{\nu}, \mathbb{S}_{\nu+\mu}
$$

are defined and constant on the whole $\mathcal{U}_{\epsilon_{0}}(0)$. They coincide with the Stokes matrices $\stackrel{\circ}{\mathbb{S}}_{\nu}$, $\stackrel{\mathfrak{S}}{\nu+\mu}$ of the specific fundamental solutions $\stackrel{\circ}{Y}_{\sigma}(z)$ of the system (17.2)

$$
\frac{d Y}{d z}=\widehat{A}(z, 0) Y
$$

which satisfy $\stackrel{\circ}{Y}_{\sigma}(z) \equiv Y_{\sigma}(z, 0)$, according to Corollary 14.2. Any central connection matrix $C_{\nu}^{(0)}$ is defined and constant on the whole $\mathcal{U}_{\epsilon_{0}}(0)$, coinciding with a matrix $\dot{C}_{\nu}^{(0)}$ defined by the relation

$$
\stackrel{\circ}{Y}_{\nu}(z)=\stackrel{\circ}{Y}^{(0)}(z) \stackrel{\circ}{C}_{\nu}^{(0)}
$$


where $\stackrel{\circ}{Y}^{(0)}(z)$ is a fundamental solution of (17.2) in the Levelt form (17.3), and $\stackrel{\circ}{Y}_{\nu}(z)=Y_{\nu}(z, 0)$ as above.

The matrix entries of Stokes matrices vanish in correspondence with coalescing eigenvalues, i.e.

$$
\left(\mathbb{S}_{1}\right)_{i j}=\left(\mathbb{S}_{1}\right)_{j i}=\left(\mathbb{S}_{2}\right)_{i j}=\left(\mathbb{S}_{2}\right)_{j i}=0 \quad \text { whenever } u_{i}(0)=u_{j}(0) \text {. }
$$

Corollary 19.2. (Corollary 1.1) If moreover the diagonal entries of $\widehat{A}_{1}(0)$ do not differ by non-zero integers, Corollary 4.1 applies. Accordingly, there is a unique formal solution of the system with $t=0$, whose coefficients are necessarily

$$
\stackrel{\circ}{F}_{k} \equiv F_{k}(0) \text {. }
$$

Hence, there exists only one choice of fundamental solutions $\stackrel{\circ}{Y}_{\sigma}(z)$ 's with canonical asymptotics at $z=\infty$ corresponding to the unique formal solution, which necessarily coincide with the $Y_{\sigma}(z, 0)$ 's.

Summarizing, the monodromy data are computable from the system with fixed $t=0$ and are:

- $J^{(0)}=$ a Jordan form of $\widehat{A}_{1}(0) ; R^{(0)}=\stackrel{\circ}{R}$. See Proposition 17.1 .

- $B_{1}=\operatorname{diag}\left(\widehat{A}_{1}(0)\right)$.

- $\mathbb{S}_{\nu}=\stackrel{\leftrightarrow}{S}_{\nu}, \mathbb{S}_{\nu+\mu}=\stackrel{\triangleright}{S}_{\nu+\mu}$.

- $C_{\nu}^{(0)}=\dot{C}_{\nu}^{(0)}$.

Here, $\stackrel{\circ}{S}_{1}$ and $\stackrel{\circ}{S}_{2}$ are the Stokes matrices of those fundamental solutions $\dot{Y}_{1}(z), \stackrel{\circ}{Y}_{2}(z), \stackrel{\circ}{Y}_{3}(z)$ of the system (17.2) (i.e. system (1.21)) with the specific canonical asymptotics (1.23) satisfying $\stackrel{\circ}{F}_{k} \equiv F_{k}(0)$, $k \geq 1$. For these solutions the identity $\dot{Y}_{r}(z)=Y_{r}(z, 0)$ holds. In case of Lemma 19.2, only these solutions exist.

\section{Isomonodromy Deformations with Vanishing Conditions on Stokes Matrices, Proof OF THEOREM 1.2}

We now consider again system (1.9) with eigenvalues (1.15) coalescing at $t=0$, but we give up the assumption that $\widehat{A}_{1}(t)$ is holomorphic in the whole $\mathcal{U}_{\epsilon_{0}}(0)$. We assume that $\widehat{A}_{1}(t)$ is holomorphic on a simply connected open domain $\mathcal{V} \subset \mathcal{U}_{\epsilon_{0}}(0)$, as in Definition 1.1, so that the Jimbo-Miwa-Ueno isomonodromy deformation theory ${ }^{27}$ is well defined $\mathcal{V}$. Therefore $Y_{\nu+k \mu}(t, z)$ 's $(k \in \mathbb{Z})$ and $Y^{(0)}(t, z)$ satisfy the system

$$
\begin{aligned}
\frac{d Y}{d z} & =\left(\Lambda(t)+\frac{\widehat{A}_{1}(t)}{z}\right) Y, \\
d Y & =\Omega(z, t) Y,
\end{aligned}
$$

and $\widehat{A}_{1}(t)$ solves the non-linear isomonodromy deformation equations

$$
\begin{aligned}
& d \widehat{A}=\frac{\partial \Omega}{\partial z}+[\Omega, \widehat{A}], \\
& d G^{(0)}=\Theta^{(0)} G^{(0)} .
\end{aligned}
$$

Here $\Omega$ and $\Theta^{(0)}$ are the same as in the previous sections, defined for $t \in \mathcal{V}$.

Since the deformation is admissible, there exists $\widetilde{\tau}$ such that $\overline{\mathcal{V}} \subset c$, where $c$ is a $\widetilde{\tau}$-cell in $\mathcal{U}_{\epsilon_{0}}(0)$. The Stokes rays of $\Lambda(0)$ will be numerated so that $\tau_{\nu}<\widetilde{\tau}<\tau_{\nu+1}$.

As in Remark 19.1, the solutions $\widehat{A}_{1}(t)$, any $Y_{\nu+k \mu}(t, z)^{\prime}$ s and $Y^{(0)}(t, z)$ of the above isomonodromy deformation equations, initially defined in $\mathcal{V}$, can be $t$-analytically continued on the universal covering of $\mathbb{C}^{n} \backslash \Delta_{\mathbb{C}^{n}}$, as a meromorphic functions. The coalescence locus $\Delta_{\mathbb{C}^{n}}$ is a locus of fixed singularities [55], so that it may be a branching locus for $\widehat{A}_{1}(t)$ and for any of the fundamental matrices $Y(z, t)$ of $(20.1)$

\footnotetext{
${ }^{27}$ The fact that $\widehat{A}_{1}$ may have eigenvalues differing by integers does not constitute a problem; see the proof of Theorem 18.1.
} 
(i.e. of (1.9)). Notice that our $\Delta$ is obviously contained in $\Delta_{\mathbb{C}^{n}}$. The movable singularities of $\widehat{A}_{1}(t)$, $Y_{\nu+k \mu}(t, z)$ and $Y^{(0)}(t, z)$ outside $\Delta_{\mathbb{C}^{n}}$ are poles and constitute, according to [55], the locus of zeros of the Jimbo-Miwa-Ueno isomonodromic $\tau$-function. This locus can also be called Malgrange's divisor, since it has been proved in [57] that it coincides with a divisor, introduced by Malgrange (see [50] [51] [52]), where a certain Riemann-Hilbert problem fails to have solution (below, we formulate a Riemann-Hilbert problem in proving Lemma 20.2). This divisor has a complex co-dimension equal to 1 , so it does not disconnect $\mathbb{C}^{n} \backslash \Delta_{\mathbb{C}^{n}}$ and $\mathcal{U}_{\epsilon_{0}}(0) \backslash \Delta$.

The fundamental solutions $Y_{\nu+k \mu}(t, z)$ 's above are the unique solutions which have for $t \in \mathcal{V}$ the asymptotic behaviour

$$
Y_{\nu+k \mu}(z, t) e^{-\Lambda(t) z} z^{-B_{1}} \sim I+\sum_{j \geq 1} F_{j}(t) z^{-j}, z \rightarrow \infty \text { in } \mathcal{S}_{\nu+k \mu}(t) .
$$

The $t$-independent Stokes matrices are then defined by the relations

$$
Y_{\nu+(k+1) \mu}(t, z)=Y_{\nu+k \mu}(t, z) \mathbb{S}_{\nu+k \mu} .
$$

Notice that also the coefficients $F_{j}(t)$ are analytically continued as meromorphic multivalued matrix functions. For the sake of the proof of the Lemma 20.1 below, the analytic continuation of $Y_{\nu+k \mu}(t, z)$ will be denoted by

$$
\mathbb{Y}_{\nu+k \mu}(z, \tilde{t})
$$

where $\tilde{t}$ is a point of the universal covering $\mathcal{R}\left(\mathcal{U}_{\epsilon_{0}}(0) \backslash \Delta\right)$, whose projection is $t$. The analytic continuation of $F_{j}(t)$ will be simply denoted by $F_{j}(\tilde{t})$

By arguments similar to those in Section 13, it is seen that as $t$ varies in $c$ or slightly beyond the boundary $\partial c$, then $Y_{\nu+k \mu}(t, z)$ maintains its asymptotic behaviour, for $t$ away from the Malgrange's divisor. But when $t$ moves sufficiently far form $c$, then the asymptotic representation (20.3) is lost. The following Lemma gives the sufficient condition such that the asymptotics (20.3) is not lost by $\mathbb{Y}_{\nu+k \mu}(z, \tilde{t})$.

Lemma 20.1. Assume that the Stokes matrices satisfy the vanishing condition

$$
\left(\mathbb{S}_{\nu}\right)_{a b}=\left(\mathbb{S}_{\nu}\right)_{b a}=\left(\mathbb{S}_{\nu+\mu}\right)_{a b}=\left(\mathbb{S}_{\nu+\mu}\right)_{b a}=0,
$$

for any $1 \leq a \neq b \leq n$ such that $u_{a}(0)=u_{b}(0)$. Then the meromorphic continuation $\mathbb{Y}_{\nu+k \mu}(z, \tilde{t}), k \in \mathbb{Z}$, on the universal covering $\mathcal{R}\left(\mathcal{U}_{\epsilon_{0}}(0) \backslash \Delta\right)$ maintains the asymptotic behaviour

$$
\mathbb{Y}_{\nu+k \mu}(z, \tilde{t}) e^{-\Lambda(t) z} z^{-B_{1}} \sim I+\sum_{j \geq 1} F_{j}(\tilde{t}) z^{-j}
$$

for $z \rightarrow \infty$ in $\widehat{\mathcal{S}}_{\nu+k \mu}(t)$ and any $\tilde{t} \in \mathcal{R}\left(\mathcal{U}_{\epsilon_{0}}(0) \backslash \Delta\right)$ away from the Malgrange's divisor. Moreover,

$$
\mathbb{Y}_{\nu+(k+1) \mu}(z, \tilde{t})=\mathbb{Y}_{\nu+k \mu}(z, \tilde{t}) \mathbb{S}_{\nu+k \mu}
$$

Here $\widehat{\mathcal{S}}_{\nu+k \mu}(t)$ is the sector in Definition 14.1.

Remark 20.1. Notice that $B_{1}=\operatorname{diag}\left(\widehat{A}_{1}(t)\right)$ is independent of $t \in \mathcal{V}$ by assumption, and $\widehat{A}_{1}(t)$ is meromorphic, so $B_{1}$ is constant everywhere. Moreover, the relation $\mathbb{S}_{\nu+2 \mu}=e^{-2 \pi i B_{1}} \mathbb{S}_{\nu} e^{2 \pi i B_{1}}$ implies that (20.4) holds for any $\mathbb{S}_{\nu+k \mu}, k \in \mathbb{Z}$.

Proof: Since $\overline{\mathcal{V}}$ belongs to the $\widetilde{\tau}$-cell $c$, then $Y_{\nu+k \mu}(z, t)$ can be denoted by $Y_{\nu+k \mu}(z, t ; \widetilde{\tau}, c)$, as in Theorem 15.1, for $t \in \mathcal{V}$ and for any $t \in c$ away from the Malgrange's divisor. Noticing that the Malgrange's divisor does not disconnect $\mathcal{U}_{\epsilon_{0}}(0) \backslash \Delta$, we proceed exactly as in the proof of Theorem 15.1. Now $\mathcal{V}$ is considered as lying on a sheet of the covering $\mathcal{R}\left(\mathcal{U}_{\epsilon_{0}}(0) \backslash \Delta\right)$. The relation (15.2) holds unchanged, and reads

$$
Y_{\nu+\mu}\left(z, \tilde{t} ; \widetilde{\tau}, c^{\prime}\right)=\mathbb{Y}_{\nu+\mu}(z, \tilde{t}, \widetilde{\tau}, c) \mathbb{K}^{[a b]} .
$$

On the other hand, the relation (15.3) becomes

$$
\mathbb{X}_{\nu+\mu}(z, \tilde{t})=Y_{\nu+\mu}\left(z, \tilde{t} ; \tilde{\tau}, c^{\prime}\right) \widetilde{\mathbb{K}}^{[a b]}(t),
$$

where $\mathbb{X}_{\nu+\mu}(z, \tilde{t})$ is a solution of the system (20.1) with coefficient $\widehat{A}_{1}(\tilde{t})$, where $\tilde{t}$ is a point of the universal covering, reached along $\gamma_{a b}$ after $R_{a b}(t)$ has crossed $R(\widetilde{\tau}-\pi)$ in Figure $33 . \mathbb{X}_{\nu+\mu}(z, \tilde{t})$ is the 
unique fundamental matrix solution having asymptotic behaviour

$$
\mathbb{X}_{\nu+\mu}(z, \tilde{t}) e^{-\Lambda(t) z} z^{-B_{1}} \sim I+\sum_{j \geq 1} F_{j}(\tilde{t}) z^{-j},
$$

in $\mathcal{S}_{\nu+\mu}(t)$. Then (15.4) is replaced by

$$
\mathbb{X}_{\nu+\mu}\left(z, \gamma_{a b} t\right)=\mathbb{Y}_{\nu+\mu}\left(z, \gamma_{a b} t\right) \mathbb{K}^{[a b]} \widetilde{\mathbb{K}}^{[a b]}, \quad t \in c .
$$

Here, $\mathbb{Y}_{\nu+\mu}\left(z, \gamma_{a b} t\right)$ is the continuation of $Y_{\nu+\mu}(z, t) \equiv Y_{\nu+\mu}(z, t ; \widetilde{\tau}, c)$ at

$$
\tilde{t}=\gamma_{a b} t .
$$

The proof that $\mathbb{K}^{[a b]}=\widetilde{\mathbb{K}}^{[a b]}=I$ holds unchanged, following from (20.4). Therefore,

$$
\mathbb{X}_{\nu+\mu}\left(z, \gamma_{a b} t\right)=\mathbb{Y}_{\nu+\mu}\left(z, \gamma_{a b} t\right)
$$

This proves that the analytic continuation $\mathbb{Y}_{\nu+\mu}(z, \tilde{t})$ along $\gamma_{a b}$ maintains the canonical asymptotic behaviour. Moreover, the ray $R_{a b}$ plays no role in the asymptotics, as it follows from (20.5) with $\mathbb{K}^{[a b]}=I$. Repeating the construction for all possible loops $\gamma_{a b}$, as in the proof of Theorem 14.1 and Theorem 15.1, we conclude that $\mathbb{Y}_{\nu+\mu}(z, \tilde{t})$ maintains its the canonical asymptotic representation for any $\tilde{t}$ in the universal covering ( $\tilde{t}$ away from the Malgrange divisor), when $z \rightarrow \infty$ in $\widehat{S}_{\nu+\mu}(t)$.

In Lemma 20.1, we have taken into account the fact that $\Delta$ is expected to be a branching locus, so that $\mathbb{Y}(z, \tilde{t})$ is defined on $\mathcal{R}\left(\mathcal{U}_{\epsilon_{0}}(0) \backslash \Delta\right)$, as the result of [55] predicts. In fact, it turns out that (20.4) implies that there is no branching at $\Delta$, as the following lemma states.

Lemma 20.2. If (20.4) holds, then:

- The meromorphic continuation on the universal covering $\mathcal{R}\left(\mathcal{U}_{\epsilon_{0}}(0) \backslash \Delta\right)$ of any $Y_{\nu+k \mu}(z, t), k \in \mathbb{Z}$, and $Y^{(0)}(z, t)$ is single-valued on $\mathcal{U}_{\epsilon_{0}}(0) \backslash \Delta$.

- The meromorphic continuation of $\widehat{A}_{1}(t)$ is single-valued on $\mathcal{U}_{\epsilon_{0}}(0) \backslash \Delta$.

In other words, $\Delta$ is not a branching locus.

The single-valued continuation of $Y_{\nu+k \mu}(z, t)$ will be simply denoted by $Y_{\nu+k \mu}(z, t)$ in the remaining part of this section, so we will no longer need the notation $\mathbb{Y}_{\nu+k \mu}(z, \tilde{t})$.

Proof of Lemma 20.2: Let $t \in \mathcal{V}$ be an admissible isomonodromic deformation and $\widehat{A}_{1}(t)$ be holomorphic in $\mathcal{V}$. Let $\widetilde{\tau}$ be the direction of an admissible ray for $\Lambda(0)$ such that $\mathcal{V}$ lies in a $\widetilde{\tau}$-cell. Since the linear relation (1.15)

$$
u_{i}(t)=u_{i}(0)+t_{i}, \quad 1 \leq i \leq n,
$$

holds, we will use $u$ as variable in place of $t$. Accordingly, we will write $\Lambda(u)$ instead of $\Lambda(t)$ and $Y(z, u)$ instead of $Y(z, t)$. Now, the fundamental solutions $Y_{\nu+k \mu}(z, u)$ and $Y^{(0)}(z, u)$ are holomorphic functions of $u \in \mathcal{V}$. We construct a Riemann-Hilbert boundary value problem (abbreviated by R-H) satisfied by ${ }^{28}$ $Y_{\nu-\mu}(z, u), Y_{\nu}(z, u), Y_{\nu+\mu}(z, u)$ and $Y^{(0)}(z, u)$.

The given data are the essential monodromy data (see Definition 17.2) $\mathbb{S}_{\nu-\mu}, \mathbb{S}_{\nu}, B_{1}, \mu_{1}, \ldots, \mu_{n}, R^{(0)}$ and $C_{\nu}^{(0)}$. Instead of $\mu_{1}, \ldots, \mu_{n}, R^{(0)}$, we can use $D^{(0)}$ and $L^{(0)}$ (see (16.2) and Remark 16.2). They satisfy a constraint, because the monodromy $\left(C_{\nu}^{(0)}\right)^{-1} e^{2 \pi i L^{(0)}} C_{\nu}^{(0)}$ at $z=0$ can be expressed in the equivalent way $e^{2 \pi i B_{1}}\left(\mathbb{S}_{\nu} \mathbb{S}_{\nu+\mu}\right)^{-1}$. Recalling that $\mathbb{S}_{\nu+\mu}=e^{-2 \pi i B_{1}} \mathbb{S}_{\nu-\mu} e^{2 \pi i B_{1}}$, the constraint is

$$
\mathbb{S}_{\nu-\mu}^{-1} e^{2 \pi i B_{1}} \mathbb{S}_{\nu}^{-1}=\left(C_{\nu}^{(0)}\right)^{-1} e^{2 \pi i L^{(0)}} C_{\nu}^{(0)} .
$$

The following relations hold for fundamental solutions:

$$
\begin{aligned}
& Y_{\nu}(z, u)=Y_{\nu-\mu}(z, u) \mathbb{S}_{\nu-\mu}, \\
& Y_{\nu+\mu}(z, u)=Y_{\nu}(z, u) \mathbb{S}_{\nu}, \\
& Y_{\nu}(z, u)=Y^{(0)}(z, u) C_{\nu}^{(0)}, \\
& Y_{\nu+\mu}(z, u)=Y^{(0)}(z, u) C_{\nu}^{(0)} \mathbb{S}_{\nu} .
\end{aligned}
$$

\footnotetext{
${ }^{28}$ Recall that $Y_{\nu+2 k \mu}\left(z e^{2 k \pi i}\right)=Y_{\nu}(z) e^{2 k \pi i B_{1}}, k \in \mathbb{Z}$
} 


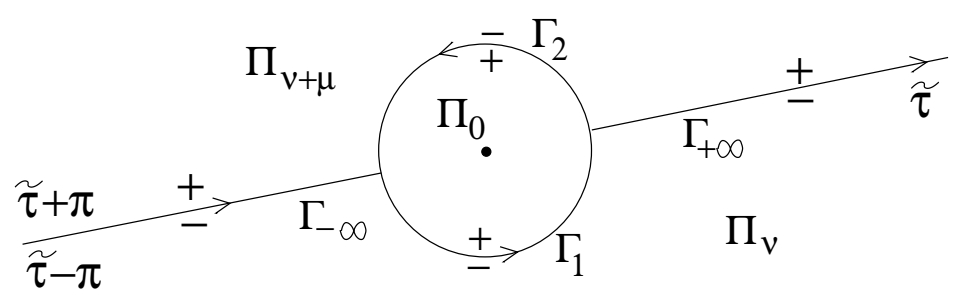

FIgURE 35. The contour $\Gamma_{-\infty} \cup \Gamma_{1} \cup \Gamma_{2} \cup \Gamma_{+\infty}$ of the Riemann-Hilbert problem, which divides the plane in regions $\Pi_{\nu}, \Pi_{\nu+\mu}$ and $\Pi_{0}$. The directional angles $\widetilde{\tau}, \widetilde{\tau} \pm \pi$ and the orientations are depicted.

Since $Y_{\nu+\mu}\left(z e^{2 \pi i}\right)=Y_{\nu-\mu}(z) e^{2 \pi i B_{1}}$, we can rewrite (20.7) as

$$
Y_{\nu}(z, u)=Y_{\nu+\mu}\left(z e^{2 \pi i}, u\right) e^{-2 \pi i B_{1}} \mathbb{S}_{\nu-\mu}
$$

We now write

$$
\begin{aligned}
& Y_{\nu+k \mu}(z, u)=\mathcal{G}_{\nu+k \mu}(z, u) e^{Q(z, u)}, \quad Q(z, u):=\Lambda(u) z+B_{1} \ln z \\
& \mathcal{G}_{\nu+k \mu}(z, u) \sim I+\sum_{j=1}^{\infty} F_{j}(u) z^{-j}, \quad z \rightarrow \infty \text { in } \mathcal{S}_{\nu+k \mu}(u), k=0,1 . \\
& Y^{(0)}(z, u)=\mathcal{G}_{0}(z, u) z^{D^{(0)}} z^{L^{(0)}} \\
& \mathcal{G}^{(0}(z, u)=G^{(0)}(u)+O(z) \quad \text { holomorphic at } z=0 .
\end{aligned}
$$

Therefore, from (20.7)-(20.11) we obtain

$$
\begin{aligned}
& \mathcal{G}_{\nu}(z, u)=\mathcal{G}_{\nu+\mu}\left(z e^{2 \pi i}, u\right) e^{Q(z, u)} \mathbb{S}_{\nu-\mu} e^{-Q(z, u)}, \\
& \mathcal{G}_{\nu+\mu}(z)=\mathcal{G}_{\nu}(z, u) e^{Q(z, u)} \mathbb{S}_{\nu} e^{-Q(z, u)} \\
& \mathcal{G}_{\nu}(z, u)=\mathcal{G}^{(0)}(z, u) z^{D^{(0)}} z^{L^{(0)}} C_{\nu}^{(0)} e^{-Q(z, u)}, \\
& \mathcal{G}_{\nu+\mu}(z, u)=\mathcal{G}^{(0)}(z, u) z^{D^{(0)}} z^{L^{(0)}} C_{\nu}^{(0)} \mathbb{S}_{\nu} e^{-Q(z, u)} .
\end{aligned}
$$

We formulate the following $\mathrm{R}-\mathrm{H}$, given the monodromy data. Consider the $z$-plane with the following branch cut from 0 to $\infty$ :

$$
\widetilde{\tau}-\pi<\arg z<\widetilde{\tau}+\pi .
$$

Consider a circle around $z=0$ of some radius $r$. The oriented contour $\Gamma=\Gamma(\widetilde{\tau})$ of the $\mathrm{R}-\mathrm{H}$ is the union of the following paths (see Figure 35):

$$
\begin{aligned}
& \Gamma_{-\infty}: \quad \arg z=\widetilde{\tau} \pm \pi,|z|>r, \text { half-line coming from } \infty \text { along the branch-cut } \\
& \Gamma_{+\infty}: \quad \arg z=\widetilde{\tau},|z|>r, \text { half-line going to } \infty \text { in direction } \widetilde{\tau}, \\
& \Gamma_{1}: \quad \widetilde{\tau}-\pi<\arg z \leq \widetilde{\tau},|z|=r, \text { half-circle in anti-clockwise sense, } \\
& \Gamma_{2}: \quad \widetilde{\tau} \leq \arg z<\widetilde{\tau}+\pi,|z|=r, \text { half-circle in anti-clockwise sense. }
\end{aligned}
$$

Recalling that $\tau_{\nu}<\widetilde{\tau}<\tau_{\nu+\mu}$, we call:

$\Pi_{\nu}$ the unbounded domain to the right of $\Gamma_{-\infty} \cup \Gamma_{1} \cup \Gamma_{+\infty}$,

$\Pi_{0}$ the ball inside the circle $\Gamma_{1} \cup \Gamma_{2}$,

$\Pi_{\nu+\mu}$ the remaining unbounded region $\mathbb{C} \backslash\left\{\Pi_{\nu} \cup \Pi_{0} \cup \Gamma\right\}$.

The $\mathrm{R}-\mathrm{H}$ problem we need is as follows:

$$
\mathcal{G}_{+}(\zeta)=\mathcal{G}_{-}(\zeta) H(\zeta, u), \quad \zeta \in \Gamma,
$$




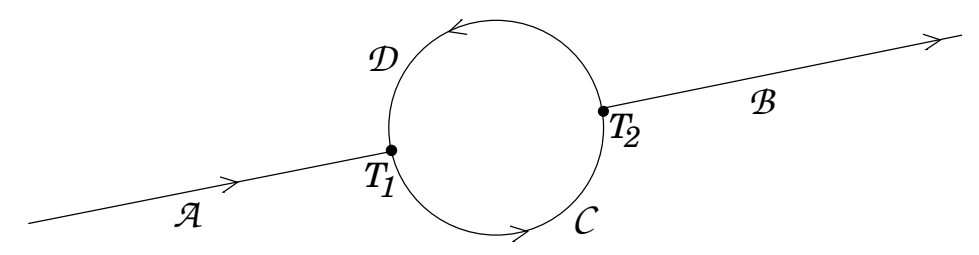

Figure 36. Jump matrices $\mathcal{A}, \mathcal{B}, \mathcal{C}, \mathcal{D}$ along $\Gamma$, used in step 1.

where the jump $H(\zeta, u)$ is uniquely specified by assigning the monodromy data $\mathbb{S}_{\nu-\mu}, \mathbb{S}_{\nu}, B_{1}, C_{\nu}^{(0)}, D^{(0)}$ and $L^{(0)}$ (i.e. $\left.\mu_{1}, \ldots, \mu_{n}, R^{(0)}\right)$. Since $\Gamma_{-}$lies along the branch-cut, we use the symbol $\zeta_{ \pm}$if $\arg \zeta=\widetilde{\tau} \pm \pi$. Hence, $H(\zeta, u)$ is

$$
\begin{aligned}
H(\zeta, u):=\quad & e^{Q\left(\zeta_{-}, u\right)} \mathbb{S}_{\nu-\mu}^{-1} e^{-Q\left(\zeta_{-}, u\right)} \quad \text { along } \Gamma_{-\infty}, \\
& e^{Q(\zeta, u)} \mathbb{S}_{\nu} e^{-Q(\zeta, u)} \quad \text { along } \Gamma_{+\infty}, \\
& e^{Q(\zeta, u)}\left(C_{\nu}^{(0)}\right)^{-1} \zeta^{-L^{(0)}} \zeta^{-D^{(0}} \quad \text { along } \Gamma_{1}, \\
& e^{Q(\zeta, u)} \mathbb{S}_{\nu}^{-1}\left(C_{\nu}^{(0)}\right)^{-1} \zeta^{-L^{(0)}} \zeta^{-D^{(0}} \quad \text { along } \Gamma_{2} .
\end{aligned}
$$

We require that the solution satisfies the conditions

$$
\begin{aligned}
& \mathcal{G}(z) \sim I+\text { series in } z^{-1}, \quad z \rightarrow \infty, z \in \Pi_{\nu} \cup \Pi_{\nu+\mu}, \\
& \mathcal{G}(z) \text { holomorphic in } \Pi_{0} \text { and } \operatorname{det}(\mathcal{G}(0)) \neq 0 .
\end{aligned}
$$

By (20.12)-(20.15), our R-H has the following solution for $u \in \mathcal{V}$ :

$$
\mathcal{G}(z, u)=\left\{\begin{aligned}
\mathcal{G}_{0}(z, u) & \text { for } z \in \Pi_{0}, \\
\mathcal{G}_{\nu}(z, u) & \text { for } z \in \Pi_{\nu}, \\
\mathcal{G}_{\nu+\mu}(z, u) & \text { for } z \in \Pi_{\nu+\mu},
\end{aligned} \quad \text { holomorphic of } u \in \mathcal{V}\right.
$$

By the result of [55], this solution can be analytically continued in $u$ as a meromorphic function on the universal covering of $\mathbb{C}^{n} \backslash \Delta_{\mathbb{C}_{n}}$. Consider a loop around $\Delta$, as in (14.5), involving two coalescing coordinates $u_{a}, u_{b}$, starting from a point in $\mathcal{V}$. We want to prove that the above continuation is single valued along this loop. As in the proof of Theorem 14.1, we just need to consider the case when $\left|u_{a}-u_{b}\right|$ is small and only $P R_{a b}$ and $P R_{b a} \operatorname{cross} l(\widetilde{\tau})$. Let

$$
\varepsilon:=u_{a}-u_{b} .
$$

The lemma will be proved if we prove that $\mathcal{G}$ in (20.19) is holomorphic in a neighbourhood of $\varepsilon=0$, except at most for a finite number of poles (the Malgrange's divisor).

In the following, we will drop $u$ and only write the dependence on $\varepsilon$. For example, we write $H(\zeta, \varepsilon)$ instead of $H(\zeta, u)$. For our convenience, as in Figure 36 we call

$$
\begin{aligned}
H(\zeta, \varepsilon) & =: \mathcal{A}\left(\zeta_{-}, \varepsilon\right) & & \text { along } \Gamma_{-\infty}, \\
& =: \mathcal{B}(\zeta, \varepsilon) & & \text { along } \Gamma_{+\infty}, \\
& =: \mathcal{C}(\zeta, \varepsilon) & & \text { along } \Gamma_{1}, \\
& =: \mathcal{D}(\zeta, \varepsilon) & & \text { along } \Gamma_{2} .
\end{aligned}
$$

$\mathcal{A}, \ldots, \mathcal{D}$ are holomorphic functions of $\varepsilon$. The following cyclic relations are easily verified:

$$
\mathcal{A}\left(z e^{-2 \pi i}, \varepsilon\right) \mathcal{D}(z, \varepsilon) \mathcal{C}\left(z e^{-2 \pi i}, \varepsilon\right)^{-1}=I \quad \mathcal{C}(z, \varepsilon) \mathcal{D}(z, \varepsilon)^{-1} \mathcal{B}(z, \varepsilon)^{-1}=I .
$$

In particular, the following "smoothness condition" holds at the points $T_{1}$ and $T_{2}$ of intersection of $\Gamma_{-\infty}$ and $\Gamma_{+\infty}$ with the circle $|z|=r$ respectively:

$$
\mathcal{A}\left(\zeta_{-}, \varepsilon\right) \mathcal{D}\left(\zeta_{+}, \varepsilon\right) \mathcal{C}\left(\zeta_{-}, \varepsilon\right)^{-1}=I \text { at } T_{1}, \quad \mathcal{C}(\zeta, \varepsilon) \mathcal{D}(\zeta, \varepsilon)^{-1} \mathcal{B}(\zeta, \varepsilon)^{-1}=I \text { at } T_{2}
$$




\section{$\stackrel{\ell_{A} \longrightarrow P_{1}}{\longrightarrow}$ \\ jump $\mathcal{A}$}

FiguRE 37. Step 2: the auxiliary Riemann-Hilbert problem with contour $\ell_{\mathcal{A}}$ and $\ell_{\mathcal{B}}$.

Indeed,

$$
\begin{aligned}
& \mathcal{A}\left(z e^{-2 \pi i}, \varepsilon\right) \mathcal{D}(z, \varepsilon) \mathcal{C}\left(z e^{-2 \pi i}, \varepsilon\right)^{-1}= \\
& =e^{Q\left(z e^{-2 \pi i}\right)} \mathbb{S}_{\nu-\mu}^{-1} e^{-Q\left(z e^{-2 \pi i}\right)} \cdot e^{Q(z)} \mathbb{S}_{\nu}^{-1}\left(C_{\nu}^{(0)}\right)^{-1} z^{-L^{(0)}} z^{-D^{(0)}} \cdot\left(z e^{-2 \pi i}\right)^{D^{(0)}}\left(z e^{-2 \pi i}\right)^{L^{(0)}} C_{\nu}^{(0)} e^{-Q\left(z e^{-2 \pi i}\right)} \\
& =e^{-2 \pi i B_{1}} e^{Q(z)} \mathbb{S}_{\nu-\mu}^{-1} e^{2 \pi i B_{1}} \mathbb{S}_{\nu}^{-1}\left(C_{\nu}^{(0)}\right)^{-1} z^{-L^{(0)}} z^{-D^{(0)}} \cdot z^{D^{(0)}} z^{L^{(0)}} e^{-2 \pi i L^{(0)}} C_{\nu}^{(0)} e^{-Q(z)} e^{2 \pi i B_{1}} \\
& =e^{-2 \pi i B_{1}} e^{Q(z)}\left(\mathbb{S}_{\nu-\mu}^{-1} e^{2 \pi i B_{1}} \mathbb{S}_{\nu}^{-1}\left(C_{\nu}^{(0)}\right)^{-1} e^{-2 \pi i L^{(0)}} C_{\nu}^{(0)}\right) e^{-Q(z)} e^{2 \pi i B_{1}}=I .
\end{aligned}
$$

In the last step, we have used (20.6). Moreover,

$$
\mathcal{C}(\zeta, \varepsilon) \mathcal{D}(z, \varepsilon)^{-1} \mathcal{B}(z, \varepsilon)^{-1}=e^{Q(z)}\left(C_{\nu}^{(0)}\right)^{-1} z^{-L^{(0)}} z^{-D^{(0)}} \cdot z^{D^{(0)}} z^{L^{(0)}} C_{\nu}^{(0)} \mathbb{S}_{\nu} e^{-Q(z)} \cdot e^{Q(z)} \mathbb{S}_{\nu}^{-1} e^{-Q(z)}=I .
$$

The last result follows from simple cancellations.

In order to complete the proof, we need the theoretical background, in particular the $L^{p}$ formulation of Riemann-Hilbert problems, found in the test-book [28], the lecture notes [39] and the papers [70] [25] (see also [22] [23] [24] and [13] [58] [68]). The proof is completed in the following steps, suggested to us by Marco Bertola.

- Step 1. We contruct a naive solution $\mathfrak{S}(z, \varepsilon)$ to the R-H, which does not satisfy the asymptotic condition (20.17). We start by defining $\mathfrak{S}(z, \varepsilon)=I$ in $\Pi_{0}$. Then, keeping into account the jumps $\mathcal{C}$ and $\mathcal{B}$ along $\Gamma_{1}$ and $\Gamma_{+\infty}$ respecively (see Figure 36 ), we have

$$
\mathfrak{S}(z, \varepsilon)=\left\{\begin{array}{cc}
I & \text { for } z \in \Pi_{0}, \\
\mathcal{C}(z, \varepsilon)^{-1} & \text { for } z \in \Pi_{\nu} \\
\mathcal{C}(z, \varepsilon)^{-1} \mathcal{B}(z, \varepsilon) & \text { for } z \in \Pi_{\nu+\mu},
\end{array}\right.
$$

On the other hand, starting with $\mathfrak{S}(z, \varepsilon)=I$ in $\Pi_{0}$ and keeping into account the jump $\mathcal{D}$ at $\Gamma_{2}$, we must have

$$
\mathfrak{S}(z, \varepsilon)=\mathcal{D}(z, \varepsilon)^{-1} \text { for } z \in \Pi_{\nu+\mu} .
$$

The second relation in (20.20) ensures that (20.22) and the last expression in (20.21) coincide. Moreover, starting with $\mathfrak{S}(z, \varepsilon)=I$ in $\Pi_{0}$ and crossing $\Gamma_{1}$ and then $\Gamma_{-\infty}$ with jumps $\mathcal{C}$ and $\mathcal{A}$, we find a third representation of $\mathfrak{S}(z, \varepsilon)$ for $z \in \Pi_{\nu+\mu}$, namely

$$
\mathfrak{S}(z, \varepsilon)=\mathcal{C}\left(z e^{-2 \pi i}, \varepsilon\right)^{-1} \mathcal{A}\left(z e^{-2 \pi i}, \varepsilon\right) \text { for } z \in \Pi_{\nu+\mu} .
$$

Now, the first relation in (20.20) ensures that (20.22) and (20.23) coincide.

- Step 2. We consider an auxiliary R-H as in Figure 37, whose boundary contour is the union of a half line $\ell_{\mathcal{A}}$ contained in $\Gamma_{-\infty}$ from $\infty$ to a point $P_{1}$ preceding $T_{1}$, and a half line $\ell_{\mathcal{B}}$ contained in $\Gamma_{+\infty}$ from a point $P_{2}$ following $T_{2}$ to $\infty$. The jump along these half lines is $H(\zeta, \varepsilon)$ (namely, $\mathcal{A}\left(\zeta_{-}, \varepsilon\right)$ and $\mathcal{B}(\zeta, \varepsilon)$ on the two half lines respectively). The $\mathrm{R}-\mathrm{H}$ is then

$$
\begin{gathered}
\Psi_{+}(\zeta)=\Psi_{-}(\zeta) H(\zeta, \varepsilon) \quad \zeta \in \ell_{\mathcal{A}} \cup \ell_{\mathcal{B}}, \\
\Psi(z) \sim I+\text { series in } z^{-1}, z \rightarrow \infty, z \in \Pi_{\nu} \cup \Pi_{\nu+\mu} .
\end{gathered}
$$

Keeping the above asymptotics into account, the $\mathrm{R}-\mathrm{H}$ is rewritten as follows:

$$
\Psi(z)=I+\int_{\ell_{\mathcal{A}} \cup \ell_{\mathcal{B}}} \frac{\Psi_{-}(\zeta)(H(\zeta, \varepsilon)-I)}{\zeta-z} \frac{d \zeta}{2 \pi i} .
$$


or, letting $\delta \Psi:=\Psi-I$ and $\delta H:=H-I$,

$$
\delta \Psi(z)=\int_{\ell_{\mathcal{A}} \cup \ell_{\mathcal{B}}} \frac{\delta \Psi_{-}(\zeta) \delta H(\zeta, \varepsilon)}{\zeta-z} \frac{d \zeta}{2 \pi i}+\int_{\ell_{\mathcal{A}} \cup \ell_{\mathcal{B}}} \frac{\delta H(\zeta, \varepsilon)}{\zeta-z} \frac{d \zeta}{2 \pi i} .
$$

We solve the problem by computing $\delta \Psi_{-}(\zeta)$, as the solution of the following integral equation (by taking the limit for $z \rightarrow z_{-}$belonging to the "-" side of $\left.\ell_{\mathcal{A}} \cup \ell_{\mathcal{B}}\right)$ :

$$
\begin{aligned}
\delta \Psi_{-}\left(z_{-}\right) & =\int_{\ell_{\mathcal{A} \cup \ell_{\mathcal{B}}}} \frac{\delta \Psi_{-}(\zeta) \delta H(\zeta, \varepsilon)}{\zeta-z_{-}} \frac{d \zeta}{2 \pi i}+\int_{\ell_{\mathcal{A}} \cup \ell_{\mathcal{B}}} \frac{\delta H(\zeta, \varepsilon)}{\zeta-z_{-}} \frac{d \zeta}{2 \pi i} \\
& \left.=C_{-}\left[\delta \Psi_{-} \delta H(\cdot, \varepsilon)\right)\right]\left(z_{-}\right)+C_{-}[\delta H(\cdot, \varepsilon)]\left(z_{-}\right) .
\end{aligned}
$$

Here $C_{-}$stands for the Cauchy boundary operator. We will write $C_{-}\left[\delta \Psi_{-} \delta H(\cdot, \varepsilon)\right]$ as $C_{-}[\bullet \delta H(\cdot, \varepsilon)] \delta \Psi_{-}$, to represent the operator $C_{-}[\bullet \delta H(\cdot, \varepsilon)]$ acting on $\delta \Psi_{-}$. We observe the following facts:

1. If $u$ is in the cell containing $\mathcal{V}$, as $\zeta \rightarrow \infty$ along $\ell_{\mathcal{A}}$ and $\ell_{\mathcal{B}}$, the off-diagonal matrix entries of the jump are exponentially small. Indeed

$$
H_{i j}(z, \varepsilon) \equiv H_{i j}(\zeta, u)=s_{i j} \exp \left\{\left(u_{i}-u_{j}\right) \zeta+\left(\left(B_{1}\right)_{i i}-\left(B_{1}\right)_{j j}\right) \ln \zeta\right\} \longrightarrow \delta_{i j} .
$$

This is due to the fact that $s_{i j}$ is either $\left(\mathcal{S}_{\nu}\right)_{i j}$ or $\left(\mathcal{S}_{\nu-\mu}^{-1}\right)_{i j}$. Thus, $\delta H_{i j} \in L^{2}\left(\ell_{\mathcal{A}} \cup \ell_{\mathcal{B}},|d \zeta|\right)$, and $C_{-}[\delta H]_{i j} \in L^{2}\left(\ell_{\mathcal{A}} \cup \ell_{\mathcal{B}},|d \zeta|\right)$. Hence, the problem is well posed in $L^{2}$, consisting in finding $\delta \Psi_{-}$ as the solution of

$$
\left(I-C_{-}[\bullet \delta H(\cdot, \varepsilon)]\right) \delta \Psi_{-}=C_{-}[\delta H(\cdot, \varepsilon)] .
$$

2. If $u$ is in the cell containing $\mathcal{V}$, by assumption both the operator and the given term in (20.27) depend holomorphically on $u$. Along the loops $\left(u_{i}-u_{j}\right) \mapsto\left(u_{i}-u_{j}\right) e^{2 \pi i}, 1 \leq i \neq j \leq n$, the property (20.26) is lost, because $u$ leaves the $\widetilde{\tau}$-cell containing $\mathcal{V}$, so that some Stokes rays cross the ray $R(\widetilde{\tau})$. On the other hand, if the vanishing condition $(20.4)$ holds, then $s_{a b}=s_{b a}=0 .{ }^{29}$ Thus, (20.26) continues to hold along the loop $\varepsilon \mapsto \varepsilon e^{2 \pi i}$. It follows that $I-C_{-}[\bullet \delta H(\cdot, \varepsilon)]$ is an analytic operator in $\varepsilon$ and the term $C_{-}[\delta H(\cdot, \varepsilon)]$ is also analytic, for $\varepsilon$ belonging to a sufficiently small closed ball $U$ centred at $\varepsilon=0$.

3. If $P_{1}$ and $P_{2}$ are sufficiently far away from the origin, we can take $\|\delta H(\cdot, \epsilon)\|_{\infty}=\sup _{\zeta \in \ell_{\mathcal{A}} \cup \ell_{\mathcal{B}}}|H(\zeta, \epsilon)|$ so small that the operator norm $\|\cdot\|$ in $L^{2}$ satisfies, for $\varepsilon \in U$,

$$
\left\|C_{-}[\bullet \delta H(\cdot, \varepsilon)]\right\| \leq\left\|C_{-}\right\|\|\delta H(\cdot, \epsilon)\|_{\infty}<1 .
$$

Here, $\left\|C_{-}\right\|$is the operator norm of the Cauchy operator. ${ }^{30}$ By $(20.28)$, the inverse exists:

$$
\left(I-C_{-}[\bullet \delta H(\cdot, \varepsilon)]\right)^{-1}=\sum_{k=1}^{+\infty}\left(C_{-}[\bullet \delta H(\cdot, \varepsilon)]\right)^{k}
$$

The series in the r.h.s. converges in operator norm and defines an analytic operator in $\varepsilon \in U$.

Using (20.29), we find the unique $L^{2}$-solution of (20.27) and then, substituting into (20.25), we find the ordinary solution $\Psi(z, \varepsilon)$ of the auxiliary problem, which is holomoprhic in $\varepsilon \in U$.

- Step 3: We construct a R-H along a closed contour with a continuous jump. Consider a "big" counter-clockwise oriented circle $\gamma$ centered at the origin and intersecting $\Gamma_{-\infty}$ at a point $Q_{1}$ preceding $P_{1}, \Gamma_{+\infty}$ at a point $Q_{2}$ following $P_{2}$. See Figure 38. If $\mathcal{G}$ is the solution to the starting problem (20.16), (20.17), (20.18), we construct a matrix-valued function $\Phi$ as follows:

$$
\begin{aligned}
\Phi:=\quad & \mathcal{G} \cdot \Psi(z, \varepsilon)^{-1}, \text { for } z \text { outside } \gamma \\
& \mathcal{G} \cdot \mathfrak{S}(z, \varepsilon)^{-1}, \text { for } z \text { inside } \gamma
\end{aligned}
$$

By constriction, $\Phi$ only has jumps along $\gamma$ :

$$
\Phi_{+}(\zeta)=\Phi_{-}(\zeta) \widetilde{H}(\zeta, \varepsilon), \quad \widetilde{H}(\zeta, \varepsilon):=\Psi(\zeta, \varepsilon) \mathfrak{S}(\zeta, \varepsilon)^{-1}
$$

\footnotetext{
${ }^{29}$ No difficulty arises from the fact that $\mathbb{S}_{\nu-\mu}^{-1}$ appears. If for simplicity we take the labelling (5.1)-(5.4), then $\mathbb{S}_{\nu-\mu}$ has diagonal blocks equal to $p_{j} \times p_{j}$ identity matrices. This structure persists on taking the inverse.

${ }^{30}$ Here we use the simple estimate $\left\|C_{-}(f \delta H)\right\|_{L^{2}} \leq\left\|C_{-}\right\|\|\delta H\|_{\infty}\|f\|_{L^{2}}$, for any $f \in L^{2}$.
} 


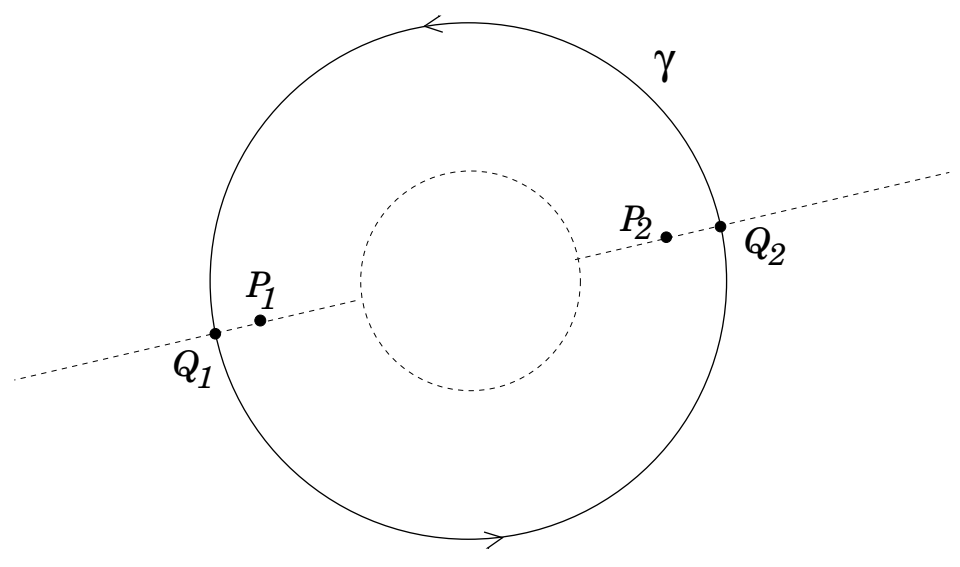

Figure 38. Step 3: the continuous Riemann-Hilbert problem on the circle $\gamma$, with jump $\Psi(\zeta, \varepsilon) \mathfrak{S}(\zeta, \varepsilon)^{-1}$.

By construction, the jump matrix $\widetilde{H}(\zeta, \varepsilon)$ is continuous in $\zeta$ along $\gamma$, and is analytic in $\varepsilon \in U$. By (20.24), then (20.17) is equivalent to

$$
\Phi(z) \sim I+\text { series in } z^{-1}, z \rightarrow \infty, z \in \Pi_{\nu} \cup \Pi_{\nu+\mu}
$$

Therefore, the R-H for $\Phi$ is solved as in (20.27) and (20.25) by

$$
\begin{aligned}
& \left(I-C_{-}[\bullet \delta \widetilde{H}(\cdot, \varepsilon)]\right) \delta \Phi_{-}=C_{-}[\delta \widetilde{H}(\cdot, \varepsilon)] \\
& \delta \Phi(z)=\int_{\gamma} \frac{\delta \Phi_{-}(\zeta) \delta \widetilde{H}(\zeta, \varepsilon)}{\zeta-z} \frac{d \zeta}{2 \pi i}+\int_{\gamma} \frac{\delta \widetilde{H}(\zeta, \varepsilon)}{\zeta-z} \frac{d \zeta}{2 \pi i}
\end{aligned}
$$

Here $C_{-}$is Cauchy operator along $\gamma$. Since $\gamma$ is a closed contour and $\widetilde{H}(\zeta, \varepsilon)$ is continuous, the procedure and results of [70] [25] [23] apply. The operator $C_{-}[\bullet \delta \widetilde{H}(\cdot, \varepsilon)]$ is Fredholm, $I-C_{-}[\bullet \delta \widetilde{H}(\cdot, \varepsilon)]$ has index 0 and its kernel is $\{0\}$. Therefore, the "analytic Fredholm alternative" of [70] holds. Namely, either $I-C_{-}[\bullet \delta \widetilde{H}(\cdot, \varepsilon)]$ can be inverted (and (20.33) can be solved) for every $\varepsilon \in U$, except for a finite number of isolated values, or is invertible for no $\varepsilon$. In the first case, $\left(I-C_{-}[\bullet \delta \widetilde{H}(\cdot, \varepsilon)]\right)^{-1}$ is meromorphic, with poles at the isolated points in $U$.

By (20.30)-(20.31), solvability of the R-H (20.32) is equivalent to the existence of the solution $\mathcal{G}(z, \varepsilon) \equiv$ $\mathcal{G}(z, u)$ for the problem $(20.16),(20.17),(20.18)$. By assumption (i.e. by the result of [55]) we know that locally in $u$ the solution $\mathcal{G}(z, u)$ exists. We therefore conclude that the "Fredholm analytic alternative" implies the existence of the solution $\Phi_{-}(\zeta, \varepsilon)$ of (20.33) for every $\varepsilon \in U$, except for a finite number of poles, and that (20.34) gives an ordinary solution $\Phi(z, \varepsilon)$, meromoprhic as a function of $\varepsilon$ in $U$. By (20.30)-(20.31), the same conclusion holds for $\mathcal{G}(z, \varepsilon) \equiv \mathcal{G}(z, u)$. This proves the Lemma (as for $\widehat{A}_{1}$, it suffices to note that $\left.\widehat{A}_{1}(t)=z\left(Y^{-1}(z, t) d Y(z, t) / d z-\Lambda(t)\right)\right)$.

Theorem 1.2 immediately follows from Lemma 20.1 and Lemma 20.2.

\section{PART V: Examples and Applications}

Our work is motivated both by the general deformation problems of linear systems with coalescing eigenvalues and by applications to Frobenius Manifolds and Painlevé equations. The applications are sketched in the sections below, which are a natural continuation of the Introduction, of which we keep the notations (for example, $\mathbb{S}_{1}, \mathbb{S}_{2}$ instead of $\mathbb{S}_{\nu+k \mu}$ ). 


\section{Monodromy Data of Semisimple Frobenius Manifolds}

A Frobenius manifold $M$ essentially is an analytic manifold with a Frobenius algebra structure on the tangent bundle and a deformed flat connection (see [17] [19] for a precise definition). The manifold is called semisimple if the algebra is semisimple in an open dense subset, whose points are called semisimple points of $M$. In suitable coordinates $u=\left(u_{1}, \ldots, u_{n}\right)$, called canonical, flatness is translated into $n+1$ compatible linear systems of dimension $n \times n$

$$
\begin{aligned}
& \frac{\partial Y}{\partial z}=\widehat{A}(z, u) Y, \quad \widehat{A}(z, u):=U+\frac{V(u)}{z} \\
& \frac{\partial Y}{\partial u_{k}}=\Omega_{k}(z, u) Y, \quad \Omega_{k}(z, u):=z E_{k}+V_{k}(u), \quad 1 \leq k \leq n .
\end{aligned}
$$

Here $E_{k}$ is the matrix with zero entries except for $\left(E_{k}\right)_{k k}=1, U=\operatorname{diag}\left(u_{1}, \ldots, u_{n}\right)$ and $V$ is skewsymmetric. The system (21.1) is of type (1.9). If we write $u=u(t)$ as in (1.15), then the following identification holds

The matrices $V_{k}$ are defined by

$$
U \equiv \Lambda(t), \quad V(u(t)) \equiv \widehat{A}_{1}(t) .
$$

$$
V_{k}(u)=\frac{\partial \Psi(u)}{\partial u_{k}} \Psi(u)^{-1}
$$

The matrix $\Psi(u)$ gives the change of basis between flat and canonical coordinates, according to the formulae in Exercise 3.2 of [19]. It is crucial for our discussion that $\Psi(u)$ is always holomorphic and invertible at semisimple points, also when $U$ has coalescing eigenvalues there. The proof is given in [15]. Therefore, the matrices $V_{k}(u)$ are holomorphic at semisimple points. $\Psi(u)$ diagonalises $V(u)$, with constant eigenvalues $\mu_{1}, \ldots, \mu_{n}$ independent of the point of the manifold (see [17],[19]):

$$
V(u)=\Psi(u) \mu \Psi(u)^{-1}, \quad \mu:=\operatorname{diag}\left(\mu_{1}, \mu_{2}, \ldots, \mu_{n}\right) .
$$

Therefore, $V(u)$ is holomorphically similar to $\mu$ at semisimple points.

The system (21.1) admits a normal form at $z=0$ such that the corresponding fundamental matrix, denoted

$$
Y_{0}(z, u)=\left(\Psi(u)+\sum_{l=1}^{\infty} \Phi_{l}(u) z^{l}\right) z^{\mu} z^{R},
$$

has monodromy exponent $R$ independent of the point of the manifold. $Y_{0}(z, u)$ is holomorphic of $u$ on the domain where $V(u)$ is holomorphic. In our notations, $R \equiv R^{(0)}$, and $Y_{0} \equiv Y^{(0)}$, as in (1.10).

The system (21.1), (21.2) is the system (1.9), (18.2) (let $t_{k} \mapsto u_{k}$ in (18.2)). The compatibility condition reads

$$
\begin{aligned}
& {\left[U, V_{k}\right]=\left[E_{k}, V\right], \Longrightarrow\left(\delta_{k i}-\delta_{k j}\right) V_{i j}=\left(u_{i}-u_{j}\right)\left(V_{k}\right)_{i j}, 1 \leq i, j, k \leq n ;} \\
& \frac{\partial V}{\partial u_{k}}=\left[V_{k}, V\right] .
\end{aligned}
$$

Equations (21.5) coincide with the isomonodromy deformation equations (18.5) and $V_{k}(u)$ coincides with the matrix (18.1).

Next, we establish the translation between our Stokes and central connection matrices and those defined in [19]. Following [19], Section 4, we consider an oriented ray $\ell_{+}(\phi):=\{z \in \mathcal{R} \mid \arg z=\phi\}$ and (for $\epsilon>0$ small) the following two sectors

$$
\Pi_{\text {right }}:=S(\phi-\pi-\epsilon, \phi+\epsilon), \quad \Pi_{\text {left }}:=S(\phi-\epsilon, \phi+\pi+\epsilon) .
$$

In [15], we introduce the open dense subset of points $p \in M$ such that the eigenvalues of $U$ at $p$ are pairwise distinct and no Stokes rays associated with $U$ at $p$ coincide with $\ell_{+}(\phi)$, and we call any connected component of this set an $\ell$-chamber. Let $\mathcal{V}$ be an open connected domain such that $\overline{\mathcal{V}}$ is contained in an $\ell$-chamber. For suitable $\epsilon$, we can identify ${ }^{31}$

$$
e^{-2 \pi i} \Pi_{\text {left }}=\mathcal{S}_{1}(\overline{\mathcal{V}}), \quad \Pi_{\text {right }}=\mathcal{S}_{2}(\overline{\mathcal{V}}), \quad \Pi_{\text {left }}=\mathcal{S}_{3}(\overline{\mathcal{V}}),
$$

\footnotetext{
${ }^{31}$ In the notation used in the main body of the paper,

$$
e^{-2 \pi i} \Pi_{\text {left }}=\mathcal{S}_{\nu}(\overline{\mathcal{V}}), \Pi_{\text {right }}=\mathcal{S}_{\nu+\mu}(\overline{\mathcal{V}}), \Pi_{\text {left }}=\mathcal{S}_{\nu+2 \mu}(\overline{\mathcal{V}}), \text { for } \tau_{\nu}<\widetilde{\tau}<\tau_{\nu+1} .
$$
}


where $e^{-2 \pi i} \Pi_{\text {left }}:=\left\{z \in \mathcal{R} \mid z=\zeta e^{-2 \pi i}, \zeta \in \Pi_{\text {left }}\right\}$, and $\mathcal{S}_{r}(\overline{\mathcal{V}})$ is defined in the Introduction. Let $Y_{\text {left }}(z, u), Y_{\text {right }}(z, u)$ be the unique fundamental matrix solutions having the canonical asymptotics $Y_{F}(z, u)=(I+O(1 / z)) e^{z U}$ in $\Pi_{\text {left }}$ and $\Pi_{\text {right }}$ respectively. The Stokes matrices $S$ and $S_{-}$of [19] are defined by the relations,

$$
Y_{\text {left }}(z, u)=Y_{\text {right }}(z, u) S, \quad Y_{\text {left }}\left(e^{2 \pi i} z, u\right)=Y_{\text {right }}(z, u) S_{-}, \quad z \in \mathcal{R} .
$$

The symmetries of the system (21.1) imply that $S_{-}=S^{T}$. In our notations as in (1.7), the Stokes matrices are defined by

$$
Y_{3}(z, u)=Y_{2}(z, u) \mathbb{S}_{2}, \quad Y_{2}(z, u)=Y_{1}(z, u) \mathbb{S}_{1}
$$

We identify

$$
Y_{3}(z, u)=Y_{\text {left }}(z, u), \quad Y_{2}(z, u)=Y_{\text {right }}(z, u)
$$

Let $B_{1}$ denote the exponent of formal monodromy ${ }^{32}$ at $z=\infty$, so that the relation $Y_{1}\left(z e^{-2 \pi i}, u\right)=$ $Y_{3}(z, u) e^{-2 \pi i B_{1}}$ holds. $^{33}$ Since $V$ is skew symmetric and $B_{1}=\operatorname{diag}(V)=0$, the above relation reduces to

$$
Y_{1}\left(z e^{-2 \pi i}, u\right)=Y_{\text {left }}(z, u) .
$$

Therefore (21.8) coincides with (21.7), with

$$
S_{-}=\mathbb{S}_{1}^{-1}, S=\mathbb{S}_{2} .
$$

The central connection matrix such that $Y_{1}=Y^{(0)} C^{(0)}$ was defined in (1.11) and in Definition 17.1. In the theory of Frobenius manifolds, such as in [15], the central connection matrix is denoted by $C$, defined by

$$
Y_{\text {right }}(z, u)=Y_{0}(z, u) C \text {. }
$$

Since $Y_{0}=Y^{(0)}, Y_{\text {right }}=Y_{2}, Y_{2}=Y_{1} \mathbb{S}_{1}$, and $\mathbb{S}_{1}^{-1}=S^{T}$, then

$$
C^{(0)}=C \mathbb{S}_{1}^{-1}=C S^{T}
$$

Summarising, monodromy data of a Frobenius manifold are $\mu, R, S, C$, versus the monodromy data $\mu_{1}, \ldots, \mu_{n}, R^{(0)}, \mathbb{S}_{1}, \mathbb{S}_{2}, C^{(0)}$ of the present paper $\left(B_{1}=0\right)$.

Coalescence points for $U$ in (21.1) are singular points for the monodromy preserving deformation equations (21.4) and (21.5). Their study is at the core of the analytic continuation of Frobenius structures. Our Theorem 1.1 allows to extend the isomonodromic approach to Frobenius manifolds at coalescence points if the manifold is semisimple at these points. Let $u^{(0)}=\left(u_{1}^{(0)}, \ldots, u_{n}^{(0)}\right)$ denote a coalescence point. By a change $Y \mapsto P Y$ in (21.1), given by a permutation matrix $P$, there is no loss of generality in assuming that

$$
\begin{array}{r}
u_{1}^{(0)}=\cdots=u_{p_{1}}^{(0)}=: \lambda_{1} \\
u_{p_{1}+1}^{(0)}=\cdots=u_{p_{1}+p_{2}}^{(0)}=: \lambda_{2} \\
\vdots \\
u_{p_{1}+\cdots+p_{s-1}+1}^{(0)}=\cdots=u_{p_{1}+\cdots+p_{s-1}+p_{s}}^{(0)}=: \lambda_{s},
\end{array}
$$

where $p_{1}, \ldots, p_{s}$ are integers such that $p_{1}+\cdots+p_{s}=n$, and $\lambda_{j} \neq \lambda_{k}$ for $j \neq k$. In order to have a correspondence with [19], as in formula (21.6) and (21.9), we take the ray $\ell_{+}(\phi)$ with

$$
\phi=\widetilde{\tau}+\pi \bmod 2 \pi,
$$

where $\widetilde{\tau}$ is the direction of an admissible ray for $U$ at the point $u^{(0)}$, as in Definition 6.2. Similarly to (14.2), we consider positive numbers $\delta_{k}$ and $\epsilon_{0}$ as follows

$$
\delta_{k}:=\frac{1}{2} \min _{j \neq k}\left\{\left|\lambda_{k}-\lambda_{j}+\rho e^{i\left(\frac{\pi}{2}-\phi\right)}\right|, \rho \in \mathbb{R}\right\}, \quad 0<\epsilon_{0}<\min _{1 \leq k \leq s} \delta_{k}
$$

\footnotetext{
${ }^{32}$ In general, a formal solution is $\left(I+\sum_{k=1}^{\infty} F_{k}(u) z^{-k}\right) z^{B_{1}} e^{z U}$, but in case of Frobenius manifolds $B_{1}=0$.

${ }^{33}$ In the notation of the main body of the paper, $Y_{r} \mapsto Y_{\nu+(r-1) \mu}, r=1,2,3, \mathbb{S}_{1} \mapsto \mathbb{S}_{\nu}, \mathbb{S}_{2} \mapsto \mathbb{S}_{\nu+\mu}$ and $Y_{\nu}\left(z_{(\nu)}\right)=$ $Y_{\nu+2 \mu}\left(z_{(\nu+2 \mu)}\right) e^{-2 \pi i L}$, where $z_{(\nu+(r-1) \mu)} \in \mathcal{S}_{\nu+(r-1) \mu}(\overline{\mathcal{V}})$ is seen as a point of $\mathcal{R}$ and not of $\mathbb{C}$.
} 
Consider the neighbourhood (polydisc) of $u^{(0)}$ defined by

$$
\mathcal{U}_{\epsilon_{0}}\left(u^{(0)}\right):=\left\{u \in \mathbb{C}^{n}|| u-u_{0} \mid \leq \epsilon_{0}\right\}
$$

and denote by $\Delta$ the coalescence locus passing through $u^{(0)}$, namely

$$
\Delta:=\left\{u(p) \in \mathcal{U}_{\epsilon_{0}}\left(u^{(0)}\right) \mid u_{i}=u_{j} \text { for some } i \neq j\right\} .
$$

If $u^{(0)}$ is a semisimple coalescence point, then the Frobenius Manifold $M$ is semisimple in $\mathcal{U}_{\epsilon_{0}}\left(u^{(0)}\right)$ for sufficiently small $\epsilon_{0}$ (if necessary, we further restrict $\epsilon_{0}$ in (21.11)). Given the above assumption of semisimplicity, then $\Psi(u)$ is holomorphic at $\Delta$ and this implies that $V(u)$ is holomorphically similar to $\mu$. Equation (21.4) for $k=i$ is $V_{i j}=\left(u_{i}-u_{j}\right)\left(V_{i}\right)_{i j}$, which implies that $V_{i j}(u)=0$ for $i \neq j$ and $u_{i}=u_{j}$. Therefore, recalling that $V\left(u^{(0)}\right)$ corresponds to $\widehat{A}_{1}(0)$, we conclude that the vanishing condition (1.20) holds true and then our Theorem 1.1 applies. We note that $\operatorname{diag}\left(V\left(u^{(0)}\right)\right)=0$, then the diagonal entries of $\widehat{A}_{1}(0)$ do not differ by non-zero integers, so that also Corollary 1.1 applies. Then, the following holds:

Theorem 1.1 as applied to Frobenius Manifolds: [More details in [15]] Let the Frobenius manifold $M$ be semisimple in a neighborhood $\mathcal{U}_{\epsilon_{0}}\left(u^{(0)}\right)$ of a coalescence point $u^{(0)}$, where $\epsilon_{0}$ satisfies (21.11). Then the constant monodromy data $\mu R, S, C$ of the manifold are well defined in the whole $\mathcal{U}_{\epsilon_{1}}\left(u^{(0)}\right)$, for any $\epsilon_{1}<\epsilon_{0}$. In particular, they are well defined at $u^{(0)}$ and at all points of $\Delta$. These data coincide with the data of the system (21.1) computed at fixed $u=u^{(0)}$, as explained above.

We recall that the monodromy data for the whole manifold can be computed by an action of the braid group (see [17], [19] and [15]) staring from the data obtained in $\mathcal{U}_{\epsilon_{1}}\left(u^{(0)}\right)$. Hence, our result allows to obtain the monodromy data for the whole manifold from the data computed at a coalescence point. This relevant fact is important in the following two cases:

a) The Frobenius structure (i.e. $V(u)$ in (21.1) ) is known everywhere, but the computation of monodromy data is extremely difficult - or impossible - at generic semisimple points where $U=$ $\operatorname{diag}\left(u_{1}, \ldots, u_{n}\right)$ has distinct eigenvalues. On the other hand, the system (21.1) at a coalescence point simplifies, so that we may be able to explicitly solve it in terms of special functions and compute $S$ and $C$. In [15] we give a detailed example of this kind for the Frobenius manifold associated with the Coxeter group $A_{3}$.

b) The Frobenius structure is explicitly known only at points where $U$ has two or more non-distinct eigenvalues. The quantum cohomology of Grassmannians $\operatorname{Gr}(k, n)$ are important examples of this case: the explicit form of $V(u)$ is known only along the small quantum cohomology, where two eigenvalues of $U$ may coincide, depending on $k$ and $n$. Indeed, coalescence of eigenvalues is the most frequent case [14]. $S$ and $C$ can be explicitly computed at the small quantum cohomology locus and Theorem 1.1 allows their extension to the whole manifold. In [15] we do explicit computations for $\operatorname{Gr}(2,4)$.

\section{Computation of Monodromy Data of Painlevé Transcendents. Example of the Algebraic Solution associated With $A_{3}$}

Theorem 1.1 provides an alternative to Jimbo's approach for the computation of the monodromy data associated with Painlevé 6 transcendents holomorphic at a critical point. The example below refers to the $A_{3}$-algebraic solution of [21]. ${ }^{34}$

Equations (21.4) and skew symmetry of $V(u)$ imply that $\sum_{i} \partial_{i} V=\sum_{i} u_{i} \partial_{i} V=0$. Thus if $n=3$,

$$
V\left(u_{1}, u_{2}, u_{3}\right) \equiv V(t), \quad t:=\frac{u_{2}-u_{1}}{u_{3}-u_{1}}
$$

Write

$$
V(t)=\left(\begin{array}{ccc}
0 & \Omega_{2} & -\Omega_{3} \\
-\Omega_{2} & 0 & \Omega_{1} \\
\Omega_{3} & -\Omega_{1} & 0
\end{array}\right)
$$

\footnotetext{
${ }^{34}$ The example, reinterpreted in the framework of Frobenius manifolds, gives the analytic computation of Stokes matrices for the $A_{3}$-Frobenius manifold.
} 
Suppose we want to study the coalescence $u_{2}-u_{1} \rightarrow 0$ in the system $(21.1)$, with $u_{3}-u_{1} \neq 0$. With the substitutions $Y(z) \mapsto e^{u_{1} z} Y(z)$, and $z \rightarrow\left(u_{3}-u_{1}\right) z$, (21.1) becomes

$$
\frac{d Y}{d z}=\left[\left(\begin{array}{ccc}
0 & 0 & 0 \\
0 & t & 0 \\
0 & 0 & 1
\end{array}\right)+\frac{V(t)}{z}\right] Y .
$$

The coalescence $u_{2}-u_{1} \rightarrow 0$ corresponds to $t \rightarrow 0$.

In equations (21.5), write $\partial V / \partial u_{k}=d V / d t \cdot \partial t / \partial u_{k}$, in order to obtain the following equivalent equations

$$
\frac{d \Omega_{1}}{d t}=\frac{1}{t} \Omega_{2} \Omega_{3}, \quad \frac{d \Omega_{2}}{d t}=\frac{1}{1-t} \Omega_{1} \Omega_{3}, \quad \frac{d \Omega_{3}}{d t}=\frac{1}{t(t-1)} \Omega_{1} \Omega_{2} .
$$

$V(t)$ can be expressed in terms of transcendents $y(t)$ satisfying the following Painlevé 6 equation, called $P V I_{\mu}$ (see [19], and also [34] for an asymptotic study of (22.2)):

$$
\frac{d^{2} y}{d t^{2}}=\frac{1}{2}\left[\frac{1}{y}+\frac{1}{y-1}+\frac{1}{y-t}\right]\left(\frac{d y}{d t}\right)^{2}-\left[\frac{1}{t}+\frac{1}{t-1}+\frac{1}{y-t}\right] \frac{d y}{d t}+\frac{1}{2} \frac{y(y-1)(y-t)}{t^{2}(t-1)^{2}}\left[(2 \mu-1)^{2}+\frac{t(t-1)}{(y-t)^{2}}\right],
$$

with parameter $\mu \in \mathbb{C}$. The eigenvalues of $V(t)$ are $\mu, 0,-\mu$. The following are the explicit formulae (see $[32])$.

$$
\begin{aligned}
\Omega_{1}=i \frac{\sqrt{y-1} \sqrt{y-t}}{\sqrt{t}}\left[\frac{A}{(y-1)(y-t)}+\mu\right], & \Omega_{2}=i \frac{\sqrt{y} \sqrt{y-t}}{\sqrt{1-t}}\left[\frac{A}{y(y-t)}+\mu\right], \\
\Omega_{3}=-\frac{\sqrt{y} \sqrt{y-1}}{\sqrt{t} \sqrt{1-t}}\left[\frac{A}{y(y-1)}+\mu\right], & A:=\frac{1}{2}\left[\frac{d y}{d t} t(t-1)-y(y-1)\right] .
\end{aligned}
$$

The branches (signs) in the square roots above are arbitrary. A change of the sign of one root implies a change of two signs in $\left(\Omega_{1}, \Omega_{2}, \Omega_{3}\right)$, which still yields a solution of $(22.2)$.

The "Painlevé transcendent" corresponding to the $A_{3}$-Frobenius manifold is the following algebraic solution of $P V I_{\mu}, \mu=-\frac{1}{4}$, obtained in [21] (there is a misprint in $t(s)$ in [21]),

$$
y(s)=\frac{(1-s)^{2}(1+3 s)\left(9 s^{2}-5\right)^{2}}{(1+s)\left(243 s^{6}+1539 s^{4}-207 s^{2}+25\right)}, \quad t(s)=\frac{(1-s)^{3}(1+3 s)}{(1+s)^{3}(1-3 s)} .
$$

As it is shown in [21], the Jimbo's monodromy data of the Jimbo-Miwa-Ueno isomonodromic Fuchsian system associated with algebraic solutions of $P V I_{\mu}$ are $\operatorname{tr}\left(M_{i} M_{j}\right)=2-S_{i j}^{2}, 1 \leq i<j \leq 3$, where $S$ is the Stokes matrix (in upper triangular form) of the corresponding Frobenius manifold. $S$ is well known [17], and $S+S^{T}$ is the Coxeter matrix of the reflection group $A_{3}$. Moreover, Jimbo's isomonodromic method [43], as applied in [21] (see also [41], [33] for holomorphic solutions) provides $\operatorname{tr}\left(M_{i} M_{j}\right)$. Here we apply Theorem 1.1 and obtain $S$ in an alternative, and probably simpler, way.

First, we take an holomorphic branch. It is obtained by letting $s \rightarrow-\frac{1}{3}$, which gives a convergent Taylor expansion at $t=0$ :

$$
y(t)=\frac{1}{2} t+\frac{13}{32} t^{2}+\frac{13}{64} t^{3}+\frac{201}{4096} t^{4}-\frac{229}{8192} t^{5}-\frac{101055}{2097152} t^{6}-\frac{167867}{4194304} t^{7}-\frac{3235319}{134217728} t^{8}+O\left(t^{9}\right) .
$$

Substitution of the parametric formulae (22.3) - or equivalently of (22.4) - into (22.2) yields (two changes of signs are allowed),

$$
\begin{aligned}
& \Omega_{1}(t)=i \sqrt{2}\left(\frac{1}{8}-\frac{1}{256} t-\frac{17}{16384} t^{2}-\frac{257}{524288} t^{3}+O\left(t^{4}\right)\right) \\
& \Omega_{2}(t)=-\frac{1}{32} t-\frac{1}{64} t^{2}-\frac{173}{16384} t^{3}+O\left(t^{4}\right) \\
& \Omega_{3}(t)=i \sqrt{2}\left(\frac{1}{8}+\frac{1}{256} t+\frac{47}{16384} t^{2}+\frac{1217}{524288} t^{3}+O\left(t^{4}\right)\right) .
\end{aligned}
$$

We observe that the following limits exist:

$$
\lim _{t \rightarrow 0} \Omega_{1}(t)=\frac{i}{4 \sqrt{2}}, \quad \lim _{t \rightarrow 0} \Omega_{2}(t)=0, \quad \lim _{t \rightarrow 0} \Omega_{3}(t)=\frac{i}{4 \sqrt{2}} .
$$


Thus, the assumptions of Theorem 1.1 hold, because $\Omega_{2}(t) \rightarrow 0$ as $t \rightarrow 0$. Also the Corollary 1.1 holds, because $\operatorname{diag}(V)=(0,0,0)$. Accordingly, the Stokes matrices corresponding to (22.4) for $|t|$ small can be computed using (22.1) at $t=0$, namely:

$$
\frac{d Y}{d z}=\left[\left(\begin{array}{ccc}
0 & 0 & 0 \\
0 & 0 & 0 \\
0 & 0 & 1
\end{array}\right)+\frac{V(0)}{z}\right] Y, \quad V(0)=\left(\begin{array}{ccc}
0 & 0 & -i \sqrt{2} / 8 \\
0 & 0 & i \sqrt{2} / 8 \\
i \sqrt{2} / 8 & -i \sqrt{2} / 8 & 0
\end{array}\right) .
$$

This system is integrable, as follows. First, we do a gauge trasformation $Y=\Psi \widetilde{Y}$, such that ${ }^{35}$

$$
\Psi^{-1} V(0) \Psi=\operatorname{diag}(-1 / 4,0,1 / 4), \quad \Psi=\left(\begin{array}{ccc}
i / 2 & 1 / \sqrt{2} & -i / 2 \\
-i / 2 & 1 / \sqrt{2} & i / 2 \\
1 / \sqrt{2} & 0 & 1 / \sqrt{2}
\end{array}\right) .
$$

Hence (22.5) becomes

$$
\frac{d \widetilde{Y}}{d z}=\left[\left(\begin{array}{ccc}
1 / 2 & 0 & 1 / 2 \\
0 & 0 & 0 \\
1 / 2 & 0 & 1 / 2
\end{array}\right)+\frac{1}{z}\left(\begin{array}{ccc}
-1 / 4 & 0 & 0 \\
0 & 0 & 0 \\
0 & 0 & 1 / 4
\end{array}\right)\right] \tilde{Y}
$$

We consider a column $\left(y_{1}, y_{2}, y_{3}\right)^{T}$ of $\tilde{Y}$, so that

$$
y_{1}^{\prime}=\frac{1}{2}\left(y_{1}+y_{3}\right)-\frac{1}{4 z} y_{1}, \quad y_{2}^{\prime}=0, \quad y_{3}^{\prime}=\frac{1}{2}\left(y_{1}+y_{3}\right)+\frac{1}{4 z} y_{3} .
$$

By elimination of $y_{3}(z)$ and setting $y_{1}(z)=\sqrt{z / 2} e^{z / 2} w(i z / 2)$ we find that the system reduces to the Bessel equation

$$
\xi^{2} \frac{d^{2} w}{d \xi^{2}}+\xi \frac{d w}{d \xi}+\left[\xi^{2}-\left(\frac{3}{4}\right)^{2}\right] w=0
$$

The last equation is integrated in terms of Hankel functions, so that we find general solutions $y_{1}(z)$ of the form

$$
y_{1}\left(z, c_{1}, c_{2}, m, n\right)=\sqrt{\frac{z}{2}} e^{\frac{z}{2}}\left(c_{1} H_{\frac{3}{4}}^{(1)}\left(\frac{i z}{2} e^{i m \pi}\right)+c_{2} H_{\frac{3}{4}}^{(2)}\left(\frac{i z}{2} e^{i n \pi}\right)\right), \quad c_{1}, c_{2} \in \mathbb{C}, m, n \in \mathbb{Z} .
$$

The Stokes rays of the system (22.5) are given by $\Re(i z)=0$, namely $\arg z=\frac{\pi}{2}+k \pi, k \in \mathbb{Z}$. Consider three canonical sectors

$$
\mathcal{S}_{1}=S\left(-\frac{3 \pi}{2}, \frac{\pi}{2}\right), \mathcal{S}_{2}=S\left(-\frac{\pi}{2}, \frac{3 \pi}{2}\right), \mathcal{S}_{3}=S\left(\frac{\pi}{2}, \frac{5 \pi}{2}\right)
$$

The asymptotic behaviour of fundamental matrices $\widetilde{Y}_{r}(z)=\Psi^{-1} Y_{r}(z)$ corresponding to canonical asymptotics of $Y_{r}(z)$ for $z \rightarrow \infty$ in $\mathcal{S}_{r}, r=1,2,3$, is of the type

$$
\tilde{Y}(z)=\Psi^{-1}\left(I+\mathcal{O}\left(\frac{1}{z}\right)\right)\left(\begin{array}{ccc}
1 & 0 & 0 \\
0 & 1 & 0 \\
0 & 0 & e^{z}
\end{array}\right)=\left(I+\mathcal{O}\left(\frac{1}{z}\right)\right)\left(\begin{array}{ccc}
-\frac{i}{2} & \frac{i}{2} & \frac{1}{\sqrt{2}} e^{z} \\
\frac{1}{\sqrt{2}} & \frac{1}{\sqrt{2}} & 0 \\
\frac{i}{2} & -\frac{i}{2} & \frac{1}{\sqrt{2}} e^{z}
\end{array}\right) .
$$

Let us compute $\mathbb{S}_{1}$, such that $Y_{2}(z)=Y_{1}(z) \mathbb{S}_{1}$. The behaviour for $z \rightarrow \infty$ of Hankel functions is

$$
\begin{aligned}
& H_{\frac{3}{4}}^{(1)}(i z / 2)=\frac{2 e^{-i \frac{7 \pi}{8}}}{\sqrt{\pi z}}\left(1+\mathcal{O}\left(\frac{1}{z}\right)\right) e^{-z / 2}, \quad-\frac{3 \pi}{2}<\arg z<\frac{3 \pi}{2}, \\
& H_{\frac{3}{4}}^{(2)}(i z / 2)=\frac{2 e^{i \frac{3 \pi}{8}}}{\sqrt{\pi z}}\left(1+\mathcal{O}\left(\frac{1}{z}\right)\right) e^{z / 2}, \quad-\frac{5 \pi}{2}<\arg z<\frac{\pi}{2},
\end{aligned}
$$

35 Each columns of $\Psi$ can be multiplied by a constant. We have chosen $\Psi$ such that $\Psi^{T} \Psi=\left(\begin{array}{lll}0 & 0 & 1 \\ 0 & 1 & 0 \\ 1 & 0 & 0\end{array}\right)$. This has a meaning in the framework of Frobenius manifolds, but is of no importance for our computation. 
It impliies that

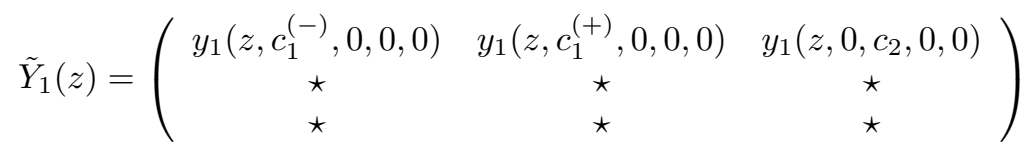

with

$$
\begin{aligned}
& y_{1}\left(z, c_{1}^{(-)}, 0,0,0\right)=c_{1}^{(-)} \sqrt{\frac{z}{2}} e^{z / 2} H_{\frac{3}{4}}^{(1)}\left(\frac{i z}{2}\right)=-\frac{i}{2}\left(1+\mathcal{O}\left(\frac{1}{z}\right)\right), \quad c_{1}^{(-)}:=-\frac{i}{2} \sqrt{\frac{\pi}{2}} e^{7 \pi i / 8} \\
& y_{1}\left(z, c_{1}^{(+)}, 0,0,0\right)=c_{1}^{(+)} \sqrt{\frac{z}{2}} e^{z / 2} H_{\frac{3}{4}}^{(1)}\left(\frac{i z}{2}\right)=\frac{i}{2}\left(1+\mathcal{O}\left(\frac{1}{z}\right)\right), \quad c_{1}^{(+)}:=\frac{i}{2} \sqrt{\frac{\pi}{2}} e^{7 \pi i / 8} \\
& y_{1}\left(z, 0, c_{2}, 0,0\right)=c_{2} \sqrt{\frac{z}{2}} e^{z / 2} H_{\frac{3}{4}}^{(2)}\left(\frac{i z}{2}\right)=\frac{e^{z}}{\sqrt{2}}\left(1+\mathcal{O}\left(\frac{1}{z}\right)\right), \quad c_{2}:=\frac{\sqrt{\pi}}{2} e^{-3 \pi i / 8} .
\end{aligned}
$$

The asymptotics of $H_{\frac{3}{4}}^{(1)}(i z / 2)$ extends up to $\arg z=3 \pi / 2$. Therefore, the first two matrix entries in the first row of $Y_{2}(z)$ are the same of $Y_{1}(z)$, which implies

$$
\mathbb{S}_{1}=\left(\begin{array}{ccc}
1 & 0 & \left(\mathbb{S}_{1}\right)_{13} \\
0 & 1 & \left(\mathbb{S}_{1}\right)_{23} \\
0 & 0 & 1
\end{array}\right)
$$

To find the third entry, we observe that $\mathcal{S}_{2}$ is obtained from $\mathcal{S}_{1}$ by a rotation $z \mapsto z e^{-i \pi}$, and that $H_{\frac{3}{4}}^{(1)}\left(i z e^{-i \pi} / 2\right)$ gives the correct asymptotics for $-\pi / 2<\arg z<5 \pi / 2$. Therefore,

$$
\tilde{Y}_{2}(z)=\left(\begin{array}{ccc}
y_{1}\left(z, c_{1}^{(-)}, 0,0,0\right) & y_{1}\left(z, c_{1}^{(+)}, 0,0,0\right) & y_{1}\left(z, \widehat{c}_{1}, 0,-1,0\right) \\
\star & \star & \star \\
\star & \star & \star
\end{array}\right),
$$

with,

$$
y_{1}\left(z, \widehat{c}_{1}, 0,-1,0\right)=\widehat{c}_{1} \sqrt{\frac{z}{2}} e^{z / 2} H_{\frac{3}{4}}^{(1)}\left(i z e^{-i \pi} / 2\right)=\frac{e^{z}}{\sqrt{2}}\left(1+\mathcal{O}\left(\frac{1}{z}\right)\right), \quad \widehat{c}_{1}:=\frac{\sqrt{\pi}}{2} e^{3 \pi i / 8} .
$$

Finally, the cyclic relation (see [69])

$$
H_{\frac{3}{4}}^{(1)}\left(e^{-i \pi} \frac{i z}{2}\right)=\sqrt{2} H_{\frac{3}{4}}^{(1)}\left(\frac{i z}{2}\right)+e^{-3 \pi i / 4} H_{\frac{3}{4}}^{(2)}\left(\frac{i z}{2}\right),
$$

together with (22.10) and (22.9), implies that

$$
y_{1}\left(z, \widehat{c}_{1}, 0,-1,0\right)=\sqrt{\frac{\pi}{2}} e^{3 \pi i / 8} \sqrt{\frac{z}{2}} e^{z / 2} H_{\frac{3}{4}}^{(1)}\left(\frac{i z}{2}\right)+y_{1}\left(z, 0, c_{2}, 0,0\right) .
$$

On the other hand, from the definition of $\mathbb{S}_{1}$ we must have

$$
y_{1}\left(z, \widehat{c}_{1}, 0,-1,0\right)=\left(\mathbb{S}_{1}\right)_{13} y_{1}\left(z, c_{1}^{(-)}, 0,0,0\right)+\left(\mathbb{S}_{1}\right)_{23} y_{1}\left(z, c_{1}^{(+)}, 0,0,0\right)+y_{1}\left(z, 0, c_{2}, 0,0\right) .
$$

Clearly, (22.7) (22.8) and (22.11) are not enough to determine $\left(\mathbb{S}_{1}\right)_{13}$ and $\left(\mathbb{S}_{1}\right)_{23}$. Thus, we analyse the second row of (22.6), to which corresponds $y_{2}(z)$. Recall that $y_{2}^{\prime}(z)=0$. Therefore, we choose $y_{2}(z)=1 / \sqrt{2}$ for the first two entries, and $y_{2}(z)=0$ for the third. This gives, for the second row of $Y_{2}=Y_{1} \mathbb{S}_{1}$ :

$$
\left[\frac{1}{\sqrt{2}}, \frac{1}{\sqrt{2}}, 0\right]=\left[\frac{1}{\sqrt{2}}, \frac{1}{\sqrt{2}},\left(\left(\mathbb{S}_{1}\right)_{13}+\left(\mathbb{S}_{1}\right)_{23}\right) \frac{1}{\sqrt{2}}\right] \Longrightarrow\left(\mathbb{S}_{1}\right)_{23}=-\left(\mathbb{S}_{1}\right)_{13} .
$$

Thus, (22.11) and (22.12) become

$$
\left(\mathbb{S}_{1}\right)_{13}\left(y_{1}\left(z, c_{1}^{(-)}, 0,0,0\right)-y_{1}\left(z, c_{1}^{(+)}, 0,0,0\right)\right)=\sqrt{\frac{\pi}{2}} e^{3 \pi i / 8} \sqrt{\frac{z}{2}} e^{z / 2} H_{\frac{3}{4}}^{(1)}\left(\frac{i z}{2}\right) .
$$


Keeping into account $(22.7)$ and $(22.8)$ we find $\left(\mathbb{S}_{1}\right)_{13}=1$. Thus

$$
\mathbb{S}_{1}=\left(\begin{array}{ccc}
1 & 0 & 1 \\
0 & 1 & -1 \\
0 & 0 & 1
\end{array}\right)
$$

$\mathbb{S}_{2}$ can be computed in a similar way, by a further rotation. On the other hands, since $V$ is skew symmetric

$$
\mathbb{S}_{2}=\mathbb{S}_{1}^{-T}=\left(\begin{array}{ccc}
1 & 0 & 0 \\
0 & 1 & 0 \\
-1 & 1 & 1
\end{array}\right)
$$

The result is in accordance with Theorem 1.1, which predicts that the entry $(1,2)$ of $\mathbb{S}_{1}$ and the entry $(2,1)$ of $\mathbb{S}_{2}$ must be zero. It is also in accordance with the monodromy data of $y(t)$ obtained in [21].

Remark 22.1. If we choose $V(0)$ with different signs, we obtain different signs in $\mathbb{S}_{1}$. For example, consider the choice

$$
\bar{V}(0)=\left(\begin{array}{ccc}
0 & 0 & -i \sqrt{2} / 8 \\
0 & 0 & -i \sqrt{2} / 8 \\
i \sqrt{2} / 8 & i \sqrt{2} / 8 & 0
\end{array}\right)=J V(0) J, \quad J:=\operatorname{diag}(1,-1,1) .
$$

The same procedure as above yields

$$
\overline{\mathbb{S}}_{1}=\left(\begin{array}{ccc}
1 & 0 & -1 \\
0 & 1 & -1 \\
0 & 0 & 1
\end{array}\right) \equiv J \mathbb{S}_{1} J
$$

This sign freedom corresponds to the invariance of $U=\operatorname{diag}\left(u_{1}, u_{2}, u_{3}\right)$, namely $J U J \equiv U$. The result $\overline{\mathbb{S}}_{1}$ above is in accordance with the known result of [17] that the Stokes matrix $S$ of the $A_{3}$ Frobenius manifold is (up to permutation, change of signs and action of the braid group) the Stokes matrix $S$ such that $S+S^{T}$ is the Coxeter matrix of the reflection group $A_{3}$.

Remark 22.2. Another Stokes matrix $S$ obtained by an action of the braid group from that computed above exists with entries $\left(S_{12}, S_{13}, S_{23}\right)=(1,1,1)$; the corresponding branch $y(t)$ at $t=0$ is obtained letting $s \rightarrow 1$, yielding the Puiseux series [21]

$$
y(t)=\frac{4^{2 / 3}}{50} t^{2 / 3}+\frac{1}{2} t+\frac{1941 \cdot 2^{2 / 3}}{2500} t^{4 / 3}-\frac{2^{1 / 3}}{150} t^{5 / 3}+O\left(t^{2}\right),
$$

to which corresponds the behaviour of $V(t)$,

$$
\begin{gathered}
\Omega_{1}(t)=\frac{2^{1 / 6}}{12 \cdot t^{1 / 6}}-\frac{5 \cdot 2^{5 / 6}}{96} t^{1 / 6}+O\left(t^{1 / 2}\right), \quad \Omega_{2}(t)=\frac{i}{6}+\frac{2^{1 / 3} i}{96} t^{2 / 3}+O\left(t^{4 / 3}\right), \\
\Omega_{3}(t)=\frac{2^{1 / 6}}{12 \cdot t^{1 / 6}}+\frac{5 \cdot 2^{5 / 6}}{96} t^{1 / 6}+O\left(t^{1 / 2}\right) .
\end{gathered}
$$

Thus, $V(t)$ has a branch point at $t=0$, no entry vanishes and both $\Omega_{1}(t)$ and $\Omega_{3}(t)$ diverge, without contradiction with Theorem 1.1 .

\section{APPENDiX: Examples of Cell Decomposition}

Example 22.1. Let

$$
\Lambda(t)=\operatorname{diag}\left(u_{1}(t), u_{2}(t), u_{3}(t)\right):=\operatorname{diag}(0, t, 1) .
$$

In this example, the coalescence locus in a neighbourhood of $t=0$ is $\{0\}$, while the global coalescence locus in $\mathbb{C}$ is $\{0,1\}$. At $t=0$ we have

$$
\arg \left(u_{1}(0)-u_{3}(0)\right)=\arg (0-1), \quad \arg \left(u_{3}(0)-u_{1}(0)\right)=\arg (1-0) .
$$

We choose $\widehat{\arg }(1)=0$, $\widehat{\arg }(-1)=\pi$. This implies that an admissible direction $\eta$ such that $\eta-2 \pi<$ $\widehat{\arg }\left(u_{i}(0)-u_{j}(0)\right)<\eta$ must satisfy

$$
\eta-2 \pi<0<\eta, \quad \eta-2 \pi<\pi<\eta \quad \Longrightarrow \quad \pi<\eta<2 \pi
$$


Cell 1

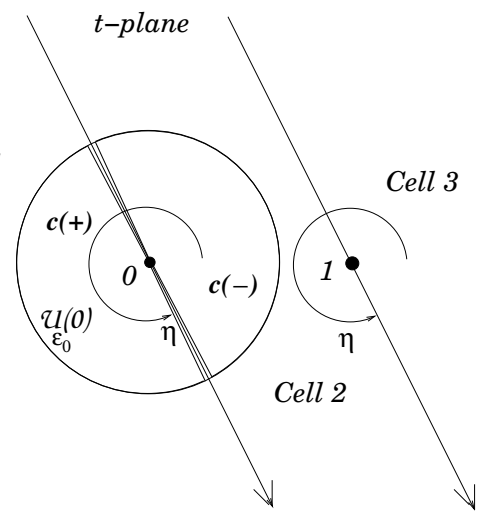

Figure 39. Cell partition (Cell 1, Cell 2, Cell 3) of the $t$-sheet $\eta-2 \pi<$ $\arg (t)<\eta$ and $\eta-2 \pi<\arg (t-1)<$ $\eta$. The neighbourhood $\mathcal{U}_{\epsilon_{0}}(0)$ (the disk) splits into two cells $c(+)$ and

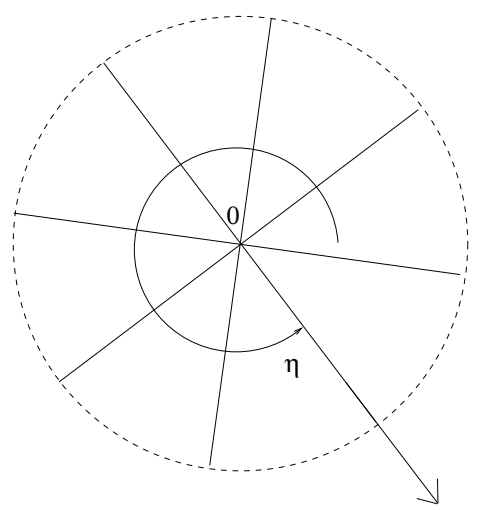

Figure 40. The cells of $\mathcal{U}_{\epsilon_{0}}(0)$ of Example 22.2. $c(-)$.

Therefore $\tau=3 \pi / 2-\eta$ satisfies

$$
-\frac{\pi}{2}<\tau<\frac{\pi}{2}
$$

- At $t \neq 0: u_{1}(t)=u_{1}(0)$ and $u_{3}(t)=u_{3}(0)$, and

$$
\begin{gathered}
\arg \left(u_{1}(t)-u_{2}(t)\right)=\arg (-t), \quad \arg \left(u_{2}(t)-u_{1}(t)\right)=\arg (t), \\
\arg \left(u_{3}(t)-u_{2}(t)\right)=\arg (1-t), \quad \arg \left(u_{2}(t)-u_{3}(t)\right)=\arg (t-1) .
\end{gathered}
$$

We impose:

$$
\begin{gathered}
\eta-2 \pi<\widehat{\arg }(-t)<\eta, \quad \eta-2 \pi<\widehat{\arg }(t)<\eta, \\
\Downarrow \\
\eta-2 \pi<\widehat{\arg }(t)<\eta-\pi \text { out } \eta-\pi<\widehat{\arg }(t)<\eta .
\end{gathered}
$$

The above gives the 2 cells of $\mathcal{U}_{\epsilon_{0}}(0)$ for $\epsilon_{0}<1$.

$$
c(-):=\left\{t \in \mathcal{U}_{\epsilon_{0}}(0) \mid \eta-2 \pi<\arg (t)<\eta-\pi\right\}, \quad c(+):=\left\{t \in \mathcal{U}_{\epsilon_{0}}(0) \mid \eta-\pi<\arg (t)<\eta\right\} .
$$

Since $u(t)$ is globally defined (and $t=1$ is another coalescence point), one can globally divide the $t$-plane into cells. Accordingly, we also impose the condition

$$
\begin{gathered}
\eta-2 \pi<\widehat{\arg }(1-t)<\eta, \quad \eta-2 \pi<\widehat{\arg }(t-1)<\eta, \\
\Downarrow \\
\eta-2 \pi<\widehat{\arg }(t-1)<\eta-\pi \text { out } \eta-\pi<\widehat{\arg }(t-1)<\eta .
\end{gathered}
$$

Therefore, the $t$ plane is globally partitioned into 3 cells by the above relation, as in figure 39 .

Example 22.2. Let

$$
\Lambda(t)=\operatorname{diag}\left(u_{1}(t), u_{2}(t), u_{3}(t), u_{4}(t), u_{5}(t)\right):=\operatorname{diag}\left(0, t, t e^{i \frac{\pi}{2}}, t e^{i \pi}, t e^{i \frac{3 \pi}{2}}\right) .
$$

The coalescence locus is $t=0$. The admissible direction $\eta$ can be chosen arbitrarily, because $\Lambda(0)=0$ has no Stokes rays. Once $\eta$ is fixed, we impose $\eta-2 \pi<\widehat{\arg }\left(u_{i}(t)-u_{j}(t)\right)<\eta$. Thus, for $0 \leq l, k \leq 3$ :

$$
\eta-2 \pi<\widehat{\arg }\left(t e^{i \frac{\pi}{2} k}\right)<\eta, \eta-2 \pi<\widehat{\arg }\left(-t e^{i \frac{\pi}{2} k}\right)<\eta, \eta-2 \pi<\widehat{\arg }\left(t\left(e^{i \frac{\pi}{2} l}-e^{i \frac{\pi}{2} k}\right)\right)<\eta .
$$

The first two constraints imply

$$
\eta-2 \pi-\frac{\pi}{2} k<\arg t<\eta-\pi-\frac{\pi}{2} k, \text { or } \eta-\pi-\frac{\pi}{2} k<\arg t<\eta-\frac{\pi}{2} k .
$$




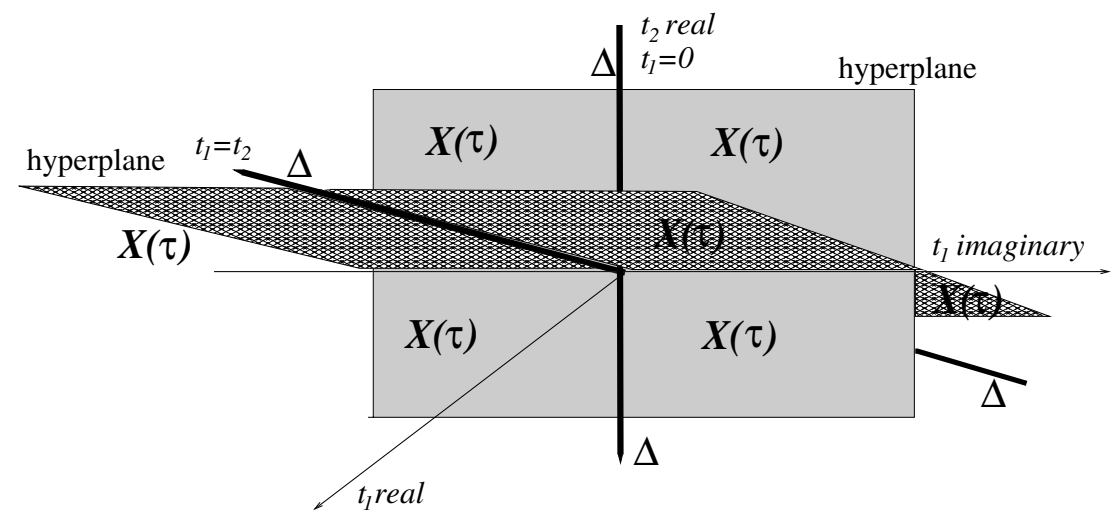

Figure 41. Example 22.3, with $\eta=3 \pi / 2$. The horizontal plane is $t_{1} \in \mathbb{C}$. The vertical axis is $t_{2} \in \mathbb{R}$. The thick lines $t_{1}=t_{2}$ (real) and $t_{1}=0\left(t_{2}\right.$ real) are the projection of $\Delta_{\mathbb{C}^{2}}$. The planes (minus $\Delta$ ) are the projection of the crossing locus $X(\tau)$. The full planes (which include the thick lines) are the projection of $W(\tau)$. They disconnect $\left\{t \in \mathbb{C}^{2} \mid t_{2} \in \mathbb{R}\right\}$.

By prosthaphaeresis formulas we have $e^{i \frac{\pi}{2} l}-e^{i \frac{\pi}{2} k}=2 i \sin \frac{\pi}{4}(l-k) e^{i \frac{\pi}{4}(l+k)}$. Therefore, the third constraint gives

$$
\eta-2 \pi-\frac{\pi}{4}(l+k)<\arg t<\eta-\pi-\frac{\pi}{4}(l+k), \text { or } \eta-\pi-\frac{\pi}{4}(l+k)<\arg t<\eta-\frac{\pi}{4}(l+k) .
$$

It turns out that the cell-partition of $\mathcal{U}_{\epsilon_{0}}(0)$ is into 8 slices of angular width $\pi / 4$, with angles determined by $\eta$. See figure 40 .

Example 22.3. We consider $t=\left(t_{1}, t_{2}\right) \in \mathbb{C}^{2}$ and $\Lambda(t)=\operatorname{diag}\left(0, t_{1}, t_{2}\right)$. The coalescence locus can be studied globally on $\mathbb{C}^{2}$ :

$$
\Delta_{\mathbb{C}^{2}}=\left\{t \in \mathbb{C}^{2} \mid t_{1}=t_{2}\right\} \cup\left\{t \in \mathbb{C}^{2} \mid t_{1}=0\right\} \cup\left\{t \in \mathbb{C}^{2} \mid t_{2}=0\right\} .
$$

This is the union of complex lines (complex dimension $=1$ ) of complex co-dimension $=1$. In particular, $t=0$ is the point of maximal coalescence. $\Lambda(0)=0$ has has no Stokes rays, thus we choose $\eta$ freely. The cell-partition for a chosen $\eta$ is given (see previous examples) by:

$$
\eta-2 \pi<\arg \left(t_{i}\right)<\eta-\pi, \text { or } \eta-\pi<\arg \left(t_{i}\right)<\eta, \quad i=1,2,
$$

and

$$
\eta-2 \pi<\arg \left(t_{1}-t_{2}\right)<\eta-\pi, \text { or } \eta-\pi<\arg \left(t_{1}-t_{2}\right)<\eta, \quad i=1,2 .
$$

In figure 41 we represent the projection of $\mathbb{C}^{2}$ onto the subspace $\left\{t \in \mathbb{C}^{2} \mid t_{2} \in \mathbb{R}\right\}$, for the choice $\eta=3 \pi / 2$. The two thick lines

$$
t_{1}=t_{2} \text { real, } \quad t_{1}=0 \text { with } t_{2} \text { real, }
$$

are the projection of $\Delta_{\mathbb{C}^{2}}$. The following planes, without the thick lines,

$$
\left\{t \mid \arg \left(t_{1}-t_{2}\right)=\frac{\pi}{2} \text { or } \frac{3 \pi}{2} \bmod 2 \pi\right\} \cup\left\{t \mid \arg \left(t_{1}\right)=\frac{\pi}{2} \text { or } \frac{3 \pi}{2} \bmod 2 \pi\right\}
$$

are the projection of the crossing locus $X(\tau)$. The planes, including the thick lines, are the projections of $W(\tau)$.

\section{REFERENCES}

[1] D.G. Babbitt, V.S. Varadarajan: Deformations of nilpotent matrices over rings and reduction of analytic families of meromorphic differential equations. Mem. Amer. Math. Soc. 55, no. 325, (1985), iv+147 pp.

[2] W.Balser, W.B.Jurkat, D.A.Lutz: Birkhoff Invariants and Stokes' Multipliers for Meromorphic Linear Differential Equations, Journal Math. Analysis and Applications, 71, 48-94, (1979).

[3] W.Balser, W.B.Jurkat, D.A.Lutz: A General Theory of Invariants for Meromorphic Differential Equations; Part I, Formal Invariants, Funkcialaj Evacioj, 22, (1979) 197-221.

[4] W.Balser, W.B.Jurkat, D.A.Lutz: A General Theory of Invariants for Meromorphic Differential Equations; Part II, Proper Invariants, Funkcialaj Evacioj, 22, (1979) 257-283. 
[5] W.Balser, W.B.Jurkat, D.A.Lutz: On the Reduction of Connection Problems for Differential Equations with an Irregular Singular Point to ones with only Regular Singularities, I. SIAM J. Math. Anal., 12, (1981).

[6] M. Bertola, M.Y. Mo: Isomonodromic deformation of resonant rational connections, IMRP Int. Math. Res. Pap. 11, (2005), 565-635.

[7] Y.P. Bibilo: Isomonodromic Deformations of systems of linear differential equations with irregular singularities, Sbornik: Mathematics 203, (2012), 826-843.

[8] P. P. Boalch: Symplectic manifolds and isomonodromic deformations. Adv. Math. 163, (2001), $137 ? 205$

[9] A.A. Bolibruch: On an analytic transformation to standard Birkhoff form. (Russian); translated from Dokl. Akad. Nauk 334, no. 5, (1994), 553-555.

[10] A. A. Bolibruch: On Isomonodromic Deformations of Fuchsian Systems, Journ. of Dynamical and Control Systems, 3, (1997), 589-604.

[11] A. A. Bolibruch: On Isomonodromic Confluence of Fuchsian Singularities, Proc. Stek. Inst. Math. 221 (1998), 117132.

[12] T. Bridgeland, V. Toeldano Laredo: Stokes factors and Multilogarithms. J. reine und angew. Math. 682 (2013), 89-128.

[13] K.F. Clancey, I. Gohberg: Factorization of matrix functions and singular integral operators. Operator Theory: Advances and Applications, 3. Birkhäuser Verlag, Basel-Boston, Mass., (1981).

[14] G. Cotti: Coalescence Phenomenon of Quantum Cohomology of Grassmannians and the Distribution of Prime Numbers, arXiv:1608.06868 (2016).

[15] G. Cotti, B.A. Dubrovin, D. Guzzetti: Local Moduli of Semisimple Frobenius Coalescent Structures, to appear.

[16] G. Cotti, B.A. Dubrovin, D. Guzzetti: to appear.

[17] B.Dubrovin: Geometry of 2D topological field theories, Lecture Notes in Math, 1620, (1996), 120-348.

[18] B. Dubrovin: Geometry and Analytic Theory of Frobenius Manifolds, arXiv, :math/9807034, (1998)

[19] B.Dubrovin: Painlevé trascendents in two-dimensional topological field theory, in "The Painlevé Property, One Century later" edited by R.Conte, Springer (1999).

[20] B. Dubrovin: On Almost Duality for Frobenius Manifolds, Geometry, topology, and mathematical physics, 75-132, Amer. Math. Soc. Transl. Ser. 2, 212, (2004).

[21] B. Dubrovin, M. Mazzocco: Monodromy of certain Painlevé transcendents and reflection groups, Invent.Math., 141, (2000), 55-147.

[22] P.A. Deift Orthogonal polynomials and random matrices: a Riemann-Hilbert approach. Courant Lecture Notes in Mathematics, 3. New York University, CIMS, New York (1999).

[23] P. Deift.; T. Kriecherbauer; K. T-R McLaughlin; S. Venakides; X. Zhou: Uniform asymptotics for polynomials orthogonal with respect to varying exponential weights and applications to universality questions in random matrix theory. Comm. Pure Appl. Math. 52 , no. 11, (1999) 1335-1425.

[24] P. Deift.; T. Kriecherbauer; K. T-R McLaughlin; S. Venakides; X. Zhou: Strong asymptotics of orthogonal polynomials with respect to exponential weights. Comm. Pure Appl. Math. 52 , no. 12, (1999), 1491-1552.

[25] P. Deift., X. Zhou: A priori $L^{p}$-estimates for solutions of Riemann-Hilbert problems. Int. Math. Res. Not. no. 40, (2002) 2121-2154

[26] A. Duval: Biconfluence et groupe de Galois. J. Fac. Sci. Univ. Tokyo Sect. IA Math. 38, no. 2, (1991), $211-223$.

[27] M.V. Fedoryuk: Isomonodromy deformations of equations with irregular singularities. Math. USSR Sbornik 71, (1992), 463-479.

[28] A. Fokas, A. Its, A. Kapaev, V. Novokshenov: Painlevé Transcendents: The Riemann-Hilbert Approach. AMS (2006).

[29] R. Garnier Sur les singularits irrégulières des équations différentielles linéaires, J. Math. Pures et Appl., 8e série, 2 (1919), 99-198

[30] A. A. Glutsyuk: Stokes operators via limit monodromy of generic perturbation. J. Dynam. Control Systems 5, no. 1, (1999), 101-135.

[31] A. A. Glutsyuk: Resonant confluence of singular points and Stokes phenomena. J. Dynam. Control Systems 10, no. $2,(2004), 253-302$.

[32] D. Guzzetti: Inverse problem and monodromy data for three-dimensional Frobenius manifolds, Math. Phys. Anal. Geom. 4, (2001), 245-291.

[33] D. Guzzetti: Matching procedure for the sixth Painlevé equation, J. Phys. A: Math. Gen. 39 (2006), 11973-1231

[34] D. Guzzetti: An asymptotic reduction of a Painlevé VI equation to a Painlevé III, J. Phys. A 44, (2011), 215203, 12 pp.

[35] J. Harnad: Dual isomonodromic deformations and moment maps to loop algebras. Comm. Math. Phys. 166 (1994), no. 2, 337-365.

[36] P-F. Hsieh, Y. Sibuya: Basic Theory of Ordinary Differential Equations. Springer (1999).

[37] P-F. Hsieh, Y. Sibuya: Note on Regular Perturbation of Linear Ordinary Differential Equations at Irregular Singular Points, Funkcial. Ekvac, 8 (1966), 99-108.

[38] J. Hurtubise, C. Lambert, C. Rousseau: Complete system of analytic invariants for unfolded differential linear systems with an irregular singularity of Poincar rank $k$. Mosc. Math. J. 14, no. 2, (2014), 309-338, 427.

[39] A.R. Its: Large $N$ asymptotics in random matrices: the Riemann-Hilbert approach. In Random matrices, random processes and integrable systems, 351-413, CRM Ser. Math. Phys., Springer, New York, 2011.

[40] C. Lambert, C. Rousseau: Complete system of analytic invariants for unfolded differential linear systems with an irregular singularity of Poincaré rank 1. Mosc. Math. J. 12, no. 1, (2012), 77-138, 215. 
[41] K. Kaneko: Painlevé VI transcendents which are meromorphic at a fixed singularity, Proc. Japan Acad. Ser. A Math. Sci. 82 (2006), no. 5, 7176.

[42] V. Katsnelson, D. Volok: Deformations of Fuchsian systems of linear differential equations and the Schlesinger system. Math. Phys. Anal. Geom. 9 (2006), no. 2, 135-186.

[43] M.Jimbo: Monodromy Problem and the Boundary Condition for Some Painlevé Trascendents. Publ. RIMS, Kyoto Univ.,18 (1982), 1137-1161.

[44] M.Jimbo, T.Miwa, K.Ueno: Monodromy Preserving Deformations of Linear Ordinary Differential Equations with Rational Coefficients (I). Physica D 2, (1981), 306.

[45] T. Kato: Perturbation Theory of Linear Operators, Springer (1980).

[46] M. Klimes: Analytic classification of families of linear differential systems unfolding a resonant irregular singularity, arXiv:1301.5228 (2013)

[47] M. Klimes: Wild monodromy action on the character variety of the fifth Painleve equation, arXiv:1609.05185, (2016).

[48] V. P. Kostov: Normal forms of unfoldings of non-Fuchsian systems. C. R. Acad. Sci. Paris Ser. I Math. 318, no. 7, (1994),623-628.

[49] P. D. Lax: Linear Algebra and its Applications, John Wiley and Sons (2007).

[50] B. Malgrange: La classification des connexions irrégulières à une variable. Mathematics and physics (Paris, 1979/1982), 381-399, Progr. Math., 37, Birkhuser Boston, Boston, MA, (1983).

[51] B. Malgrange: Sur les déformations isomonodromiques. I. Singularités régulières. Mathematics and physics (Paris, 1979/1982), 401-426, Progr. Math., 37, Birkhuser Boston, Boston, MA, (1983).

[52] B. Malgrange: Sur les déformations isomonodromiques. II. Singularités irrégulières. Mathematics and physics (Paris, 1979/1982), 427-438, Progr. Math., 37, Birkhuser Boston, Boston, MA, (1983).

[53] Yu. I. Manin: Frobenius Manifolds, Quantum Cohomology and Moduli Spaces, Amer. Math. Society, Providence, RI, (1999).

[54] M. Mazzocco: Painlevé sixth equation as isomonodromic deformations equation of an irregular system, CRM Proc. Lecture Notes, 32, Amer. Math. Soc. 219-238, (2002).

[55] T. Miwa: Painlevé Property of Monodromy Preserving Deformation Equations and the Analyticity of $\tau$ Functions, Publ. RIMS, Kyoto Univ. 17 (1981), 703-721.

[56] K. Nobuoka: Isomonodromy problem of Schlesinger equations. Proc. Japan Acad. Ser. A Math. Sci. 57 (1981), no. 10, 488-491.

[57] J. Palmer: Zeros of the Jimbo, Miwa, Ueno tau function. J. Math. Phys. 40, no. 12, (1999), 6638-6681.

[58] W. Pogorzelski: Integral equations and their applications. Vol. I. International Series of Monographs in Pure and Applied Mathematics, Vol. 88, Pergamon Press (1966).

[59] J-P. Ramis: Confluence et résurgence. J. Fac. Sci. Univ. Tokyo Sect. IA Math. 36, no. 3, (1989),703-716.

[60] B. V. Shabat: Introduction to Complex Analysis, Part II, Functions of Several Variables, AMS, (1992)

[61] C. Sabbah: Isomonodromic Deformations and Frobeinius Manifolds: An introduction, Springer (2008).

[62] Saito K.: On a linear structure of a quotient variety by a finite reflection group, Publ. Res. Inst. Math. Sci. 29 (1993), no. 4,535 ?579.

[63] Saito K.: Period mapping associated to a primitive form, Publ. RIMS 19 (1983) 1231 - 1264.

[64] Saito K., Yano T., and Sekiguchi J.: On a certain generator system of the ring of invariants of a finite reflection group, Comm. in Algebra 8(4) (1980) 373 - 408.

[65] R. Schäfke: Formal fundamental solutions of irregular singular differential equations depending upon parameters. J. Dynam. Control Systems 7, no. 4, (2001), 501?533.

[66] Y. Sibuya: Simplification of a System of Linear Ordinary Differential Equations about a Singular Point, Funkcial. Ekvac, 4 (1962), 29-56.

[67] Y. Sibuya: Perturbation of Linear ODE's at Irregular Singular Points, Funkcial. Ekvac, 11 (1968), 235-146.

[68] N. P. Vekua: Systems of singular integral equations. Edited by J. H. Ferziger. P. Noordhoff, Ltd., Groningen (1967).

[69] W Wasow: Asymptotic Expansions for Ordinary Differential Equations. Dover (1965)

[70] X. Zhou: The Riemann-Hilbert problem and inverse scattering. SIAM J. Math. Anal. 20, no. 4, (1989), 966-986. 